E. L HBSARE 


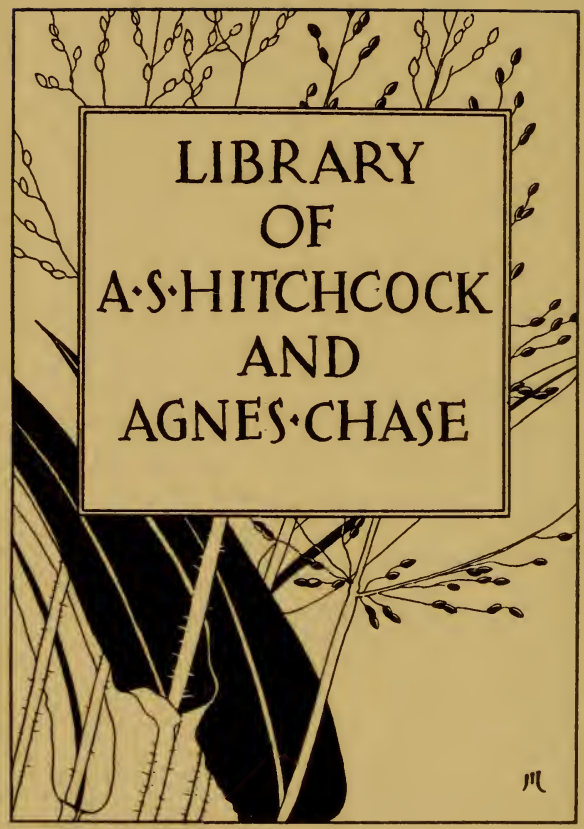



- - 


\section{BIBLIOTHÈQUE DE L'AGRICULTEUR PRATICIEN}

Encouragée par S. Exc. le Ministre de l'Agriculture

ABEILLES, Guide du propriétaire, par Collix. 4e édit. In-18, planches

ABEILLES (Education des), par A. ESPA Net. In-18.

AGRONOMIE ET PHYSIOLOGIE VÉGÉTALE. Études théoriques et pratiques

par I. Pierre 4 vol. in-18. T. Ier: Sol, Engrais, Amendemenls. 1 v. - Tome II

Plantes fourragères - Graines et produils dérivés. - Tomo III

Céréales. - Tome IV : Plantes industrielles; recherches diverses.......

ALMANACH DE L'AGRICULTEUR PRATICIEN pr 1871-72.14e année. In-18, fig

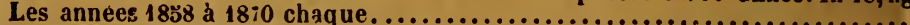

ANA

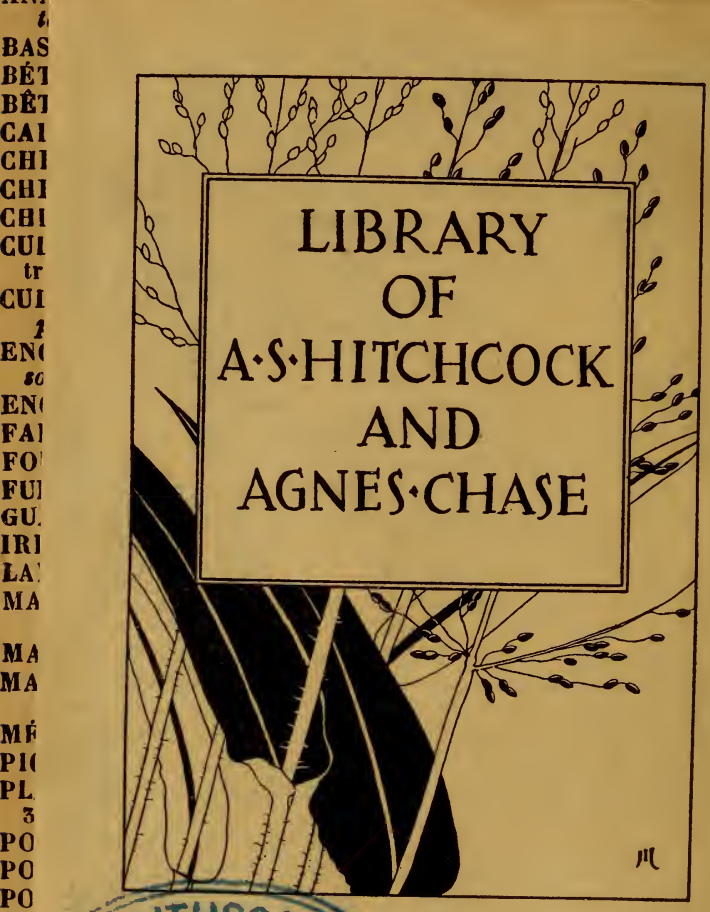

PO

西

$\mathbf{P H} / \mathrm{P}$

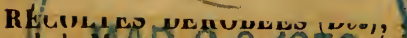

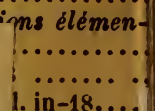

18 , fig.....

............

édit. In-18

g...........

$1-18 . . . . .$.

par Murpur

...........

(In struction

... ......

ers de pois.

.............

............

. 1 vol. in-18

$n-18 \ldots \ldots$.

..........

............

.............

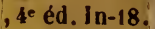

- Cidre. -

.............

irs. In-18...

pposition, va-

.............

A. 1 vol. in-18? ze édit. In-18.

ar DE TIIER.

..............

93 grav.....

1-18, 64 grav.

NET. In-18...

I Midi (Trailé

.........

in-18........

Is, et culture

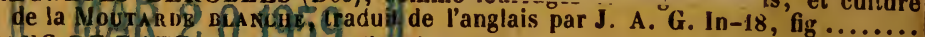
SANG DE RAT E des mhimatux d'epèces ovine et bovine, par I. Pierre. In-18... SEM A ILLES EN LIGNE (Des) et dés semoirs mécaniques, par F. GeorGes. In-18. SORGHO SHGRE, Gulture, ely, par MADINIER. In-8....................

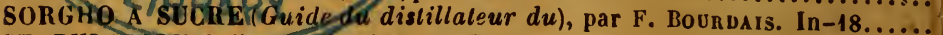
St abUlatyon de t'espece bovine, par Peers. In $-18 \ldots \ldots \ldots \ldots \ldots \ldots \ldots \ldots$ ÉGÉTAUX (Nutrit. des) dans ses rapp. avec les Assolements, par DE BA Bo. In-18. VISITE à un véritable agriculteur praticien, par Durand-Savoxat. In-18....

(1) L'Agriculteur praticien, revue de l'Agriculture française et étrangère: 24 numérosi aveo figures dans le texte. - Prix : 6 fr. - Les abonnements datent du ler janvier de chaque anpt 
Ce Catalogue annule ceux publiés précédemment.

1.84 4 1 1893

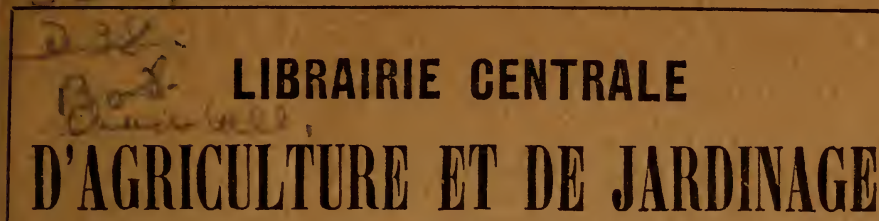

FONDEE EN 1853

16 Récompenses aux Expositions françaises

\section{CATALOGUE GÉNÉRAL}

Bibliothè $Q$ Ue de L'Agricultelr Praticien........... page 2 Abeilles, Agriculture, Amendements et engrais, Bois,

Cubage, Oiseaux de basse-cour, etc...........

BibliothèQue de L'HoRticulteur PRATIGIEN.......... Arbres fruitiers, Botanique, Culture potagere, Jardi-

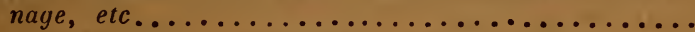

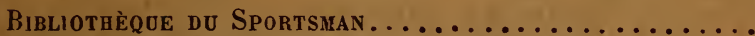

Chasse, Chevaux, Chiens, Oiseaux de chasse et de voliere,

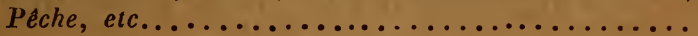

Divers. . . . . . . . . . . . . . . . . . . . . .

\section{A VIS IMPOHThing qu'on est prié de lire.}

Les commandes de livres ne sont exécutées qu'autant qu'elles sont accompagnées d'un mandat-poste. - Elles sont expédiées franco, par la poste ou par colis-postal, en gare. - Pour l'étranger, le port est à la charge des densandeurs lorsque les envois doivent être faits par chemin de fer.

Les ouvrages de Droit, de Littérature ancienne et moderne, de Médecine, de Sciences diverses, sont fournis aux mêmes conditions.

Les timbres-poste fran çais et étrangers sont rigoureusement refusés.

\section{PARIS}

Auguste GOIN, Éditeur et COMMissionNaIre RUE DES ÉCOLES, 62, PRÈS DU MUSEE DE CLUNY

La Librairie est fermée le dimanche et les jours fériés. 


\section{Bibliothèque ae I'Agricalteur praticien}

Encouragée par MII. Les Ministres ảe l'Agriculture et du Commerce et del'Instruction publique

Abeilles. Leur élevage par les procédés modernes. Pratique et théorie. Par Georges de Layens, $2^{\mathrm{e}}$ édit. 1 vol. in-18, orné de 31 figures. 150 Abeilles. Leur éducation, par Alexis Espanet. In-18. $40 \mathrm{c}$. Agriculture. - Théorie et pratique, par E. Murphy, traduit de l'anglais par SanRey. 1 vol. in-18 urné de figures. 150 Approuvé par la Commission des bibliothèques scolaires.

Angieulture moderne (Lettres sur $l^{\prime}$ ), par J. LieBig. 1 vol. in-18. 350 Aronomie. - Études théoriques et pratiques d'agronomie et de physiologie végétale, par Isidore PIErRE, doyen de la faculté des sciences de Caen. 4 vol. in-18. - $1^{\mathrm{er}}$ vol. Sol, engrais, amendements. $-2^{\mathrm{e}} \mathrm{vol}$. plantes fourragères, graines et produits dérivés. - $3^{\mathrm{e}} \mathrm{vol}$. Cèréales. $-4^{\mathrm{e}} \mathrm{vol}$. Plantes industrielles, recherches diverses. $\quad 14 \mathrm{fr}$. Ghaque volume séparément

Approuvé par la Commission des bibliothèques scolaires.

Almanach de l'agriculteur pratieien, années 1857 à 1873.16 vol. in-18 avec de nombreuses fig. dans le texte.

650

Cette collection forme une véritable Encyclopédie agricole, elle est terminée par une table générale des matières. Prix de chaque annép. $50 \mathrm{c}$.

nulyse des terres (Manuel élémentaire pour $l$ '), des amendements, des engrais liquides et solides, des eaux d'irrigation; des fourrages, des tourteaux el d'autres substances destinées à l'alimentation des hommes et des animaux, par Isidore Pierre. $2^{\circ}$ édit. 1 vol. in-18,avec fig. $\quad 250$ Approuvé par la Commission des bibliothèques scolaires.

Animaux domestiques, reproduction, amélioration et élevage, par DE WeckherLin. In-18.

$2 \mathrm{fr}$.

anthonome ales flears du Pommier; mours et destruction dz cet insecte, par M. E. Herissant, directeur de l'Ecole pratique des TroisCroix. In-18 orné d'une planche et d'un chromo représentart l'insecte à ses divers états.

$60 \mathrm{c}$.

Ce mémoire a été couronné par la Société d'Encouragement et par le Congrès pomologique de l'Ouest.

Tasse-Cour. - Poules, Oies, Canards, Pintades, Dindons, Pigeons, par le baron PEers, $2^{e}$ édit. 1 vol. in-18 et planches.

2 fr.

Bétail (De l'alimentation $d u$ ), aux points de vue de la production, du travail, de la viande, de la graisse, de la laine, du lait et des engrais, par Isidore Pierre, $4^{\mathrm{e}}$ édition. 1 vol. in-18. (Nouvelle édition en préparation.)

Approuvé par la Commission des bibliothèques scolaires.

Bêtes ovines (Traité des), par Weckherlin. 1 vol. in-18.

mêtes ovines (Des) et des Chèvres, par YsabEaU.1 vol in-18. fig. $75 \mathrm{c}$. Botte de foln $(\boldsymbol{L} a)$. - Description des plantes qu'elle peut contenir et de celles qu'on n'y doit pas trouver, par Merche, 1 vol. in-18, orné de 99 figures dans le texte. (Nouvelle édition en préparation).

Chaux, Marne et Calcuires coquilliers. Leur emploi pour l'amen-

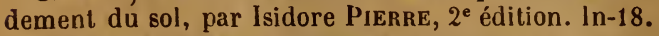

$50 \mathrm{c}$.

Constructions rurales (Manuel des), par Bona. $4^{\mathrm{e}}$ édit. 1 vol. in-18, orné de 200 fig. $\quad$ t. 350

Coqs et Poules. - Guide pratique de l'aviculteur par Desroches. 1 vol. in-18.

150

Dindons, Pintades, Dies, Canards, Cygnes, Paons, Falsans, Perdrix, Callies et Collins, par Alexis Espanet. $3^{e}$ édit. arrangée par l'éditeur. 1 vol. in-18, orné de 13 figures dans le texte.

$1 \mathrm{fr}$.

Dralnage. - Traité de drainage, ou Assainissement des terrains hu-

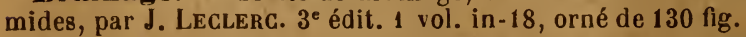

350

Engrals. - Traité des engrais (Fumier de ferme, Engrais humain, Guano, Engrais chimiques, etc.); préparation, emploi et commerce, par CHABRiER, professeur de chimie et d’agriculture d̀ Juilly, $2^{e}$ édit. 1 vol. in-18. $3 \mathrm{fr}$. 
Études agronomigues sur les féoräiques de virgile, par

A. Bosson. 1 vol. in-18.

350

Eueniyptus. - Introduction, culture, propriétés, usages, etc., par

RAVERET-IVATTEl. $2^{\circ}$ édit. 1 vol. in- 18.

Approuvé par la Commission des bibliothèques scolaires.

$15 i$

Fourrages. - Recherches sur la valeur nutritive des fourrages, par

Isidore Pierre. $4^{\circ}$ édit. 1 vol. in- 18.

250

A pprouvé par la Commissioa des bibliotbèques scolaires.

Fumfer.- Plâtrage et sulfatage du fumier et désinfection des vidanges, par Isidore Pierre, $3^{e}$ édit. In-18.

Approuvé par la Commission des bibliothèques scolaires.

$50 \mathrm{c}$.

Graminées céréales et fourragères. - Description des genres e $t$ espèces; rendement des diverses espèces, propriétés nutritives, sols qui conviennent, par DE MOOR. 1 vol. in-18, orné de 250 fig. 250

Lapin domestique (Traité pratique de l'éducation $d u$ ), par F. Alexis Espanet, 6 éd. 1 vol. in-18, orné de 15 fig. dans le texte. $1 \mathrm{fr}$. viais. - Alcoolisation des tiges du maïs et du sorgho sucré, par DURET, 1 vol. in-18. $75 \mathrm{c}$.

Matériel agricole (Le). Description et examen des instruments, machines, appareils et outils employés pour les travaux agricoles, par JourdiER. $3^{\circ}$ éd. ornée de 206 fig. dans le texte. 1 vol. in-18.

350 Mouton. - Elevage et maladies, par A. Leror. 1 vol. in-18. $2 \mathrm{fr}$. Murier. - Ses avantages et son utilité dans l'industrie, par F. Cabanis. I vol. in-18, orné d'une fig. hors texte. $2 \mathrm{fr}$. Drtie.- Ses propriétés alimentaires, médicales, agricoles et industrielles, par Eloffe. 1 vol. in-32, orné de 14 figures. $1 \mathrm{fr}$. Ortie de la chine $\left(L^{\prime}\right)$ el sa culture. - Notice sur les diverses plantes qui portent ce nom, leurs usages, par Ramon dE LA SAGRA. In-18. 1 fr. Pigeons (De l'éducation des), par Alexis Espanet. $4^{\mathrm{e}}$ édit., revue et complétée par l'éditeur. 1 vol. in-18, orné de 29 figures. $1 \mathrm{fr}$. Pomone agricole. - Plantation et culture du poirier et du pommier dans les champs et les vergers, suivie d'une notice sur la fabrication du cidre et sur la préparation alimentaire des poires et des pommes, par Ferdinand Maudurt. 1 vol. in-18, orné de 25 fig. dans le texte. 125 Ouvrage couronné par la Société centrale d’horticulture de la Seine-Inférieure. - Approuvé par la Commissiun des bibliuthèques scolaires.

Porcheries (De l'établissement des). Dispositions diverses, constructions, par Grandvoinnet, professeur à l'institut agronomique. 1 vol. orné de 95 fig.

250

Pores (Du traitement des) aux différentes époques de l'année. Extrait des meilleurs ouvrages anglais, par J. A. G. Nouvelle édition corrigée et augmentée par l'éditeur. 1 vol. in-18, orné de 65 fig.

$2 \mathrm{fr}$.

Poules et Poulets ( $\dot{E}$ ducation des), par Alexis Espanet. 4e édit. revue et corrigée par l'éditeur, 1 vol. in-18, ormé de $27 \mathrm{fig}$. $1 \mathrm{fr}$.

Prairies - Culture, iormation, entretien, amélioration, renouvele-

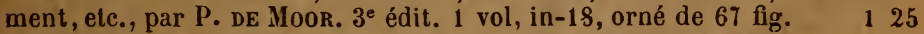

Prairles et Fourrages dans les terres fortes et argileuses du midi (Traité pratique) par ST-FÉLIX. 1804. 1 vol. in-18

$1 \mathrm{fr}$. Sang de rate des animaux d'espèces ovine et bovine, par Isidore PIERRE. In-18. - Couronné par la Société protectrice des animaux. $1 \mathrm{fr}$. Eorgho à suere (Guide du distillateur $d u$ ), par Bourdais. In-18. $1 \mathrm{fr}$. Tabac. - Culture, récolte; modes de dessication ; séchoirs, conservation, etc., par DE MOOR. $2^{e}$ édit. 1 vol. in-18, orné de $20 \mathrm{fg}$. $2 \mathrm{fr}$.

Topinambour. - Culture, alcoolisation et panification de ce tubercule, par Delbetz. 1 vol. in-18.

Approuvé par la Commission des bibliothèques scolaires.

125

végétaux (De la Nutrition des), considérée dans ses rapports avec les assolements, par le baron DE BABO. 1 vol. in-18. $1 \mathrm{fr}$. 
vigne. - Culture pratique de la vigne en Côte-d'Or, et vinification, par E. Cornu, 1 vol. in-18, orné de 22 fig. dans le texte. 150

Vigne (Nouvelle Cullure de la) en plein champ, sans échalas ni attaches, par Troullet, $4^{e}$ édit. In-18 avec 15 gravures.

vigne (Rérgénération de la), par une nouvelle plantation, par E. TrorillLET, $2^{e}$ édition. $\ln -18$. $75 \mathrm{c.}$ Abeilles, Agriculture, Amendements, Bois, Cubage, Fumiers, 0iseaux de basse-cour, Vers à soie, etc.

A bellies. - Nouvelles observations sur les abeilles, par HuBER. 2e édit. 1814; 2 vol. in-8, ornés de $14 \mathrm{pl}$.

Abeilles, leur culture, par Jannel. 1 vol. in-18, $6 \mathrm{~g}$.

$1 \mathrm{fr}$.

Abeilles. - Quelques mots sur la culture des abeilles, par D. Halleux. In-8 ${ }^{\circ}$ orné de 14 figures.

$1 \mathrm{fr}$.

Agriculture. - Cours à l'usage des praticiens et des élèves des écoles, par RAQUet. $2^{e}$ édit. 1 vol. in-18, orné de 43 fig.

Agriculture de Ia Haute-Sâ̂ne, par FASQUELLE. In-18. $3 \mathrm{fr}$.

Ariculture et horticulture des Chinols (Recherches sur $l$ ) et sur les végétaux, les animaux, et les procédés agricoles que l'on pourrait introduire avec avantage dans l'Europe occidentale et le nord de l'Afrique, par le baron d'Hervey-ST-Denis. 1830.1 vol. in- $8^{\circ}$.

$4 \mathrm{fr}$.

Agriculture pratique (Éléments $d^{\prime)}$ ou traité de la connaissance des terres, des engrais. des instruments aratoires; de la culture des céréales des plantes sarclées; textiles, oléagineuses et tinctoriales; des prairies naturelles et artificielles, par David Low, trad. par Lainé. 1838, 2 vol. in-8 ornés de $20 \dot{\text { fig. }}$.

Agriculate, Viticuliure, Horticulture et Arboriculture. Précis élémentaire et raisonné, par FenNebresque, ancien élève de Grignon. 1 vol. in-18.

2 fr.

Ajouc ou genet épineux ; culture, usages, application à la nourriture des bestiaux, par WEDLAKE. 1857. In-8.

150

Alcool de Betterave, зa fabrication par GASPARD, suivi de l'extraction de la potasse et des sels alcalins contenus dans les résidus de la distillation, par LoRmé. 1856. In-8 avec fig.

150

Amanach du Poiré et du Cidre pour 1893, par E. Vimont. 1 vol. in-18. - Prix : $1 \mathrm{fr}$.; franco 125

Animaux (Recherches expérimentales sur l'alimentation et la respiration des), par J. Allibert. In-8.

Apicuiteur ulsacien, par F. Bastian. In-18.

150

A piculture (Cours pratique $d^{\prime}$ ), professé au jardin du Luxembourg par

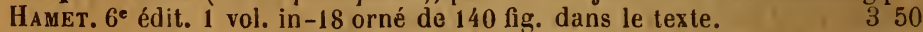

A piculture. - Petit cours, par CH. DADAnt.2e édition. 1 vol. in-18 avec planches.

$2 \mathrm{fr}$.

A piculture. - Manuel pratique d'apiculture ou résumé des travaux qu'exige le soin des abeilies, par J. Weber. In-18 orné de $33 \mathrm{fig}$. $1 \mathrm{fr}$.

Apiculture pratiofue, mise à la portée de tous les apiculteurs et augmenté de nouvelles méthodes et observations, par J. BEAUdET. 1 vol. in-i 8 , orné de planches hors texte.

Arpentage et nivellement.- Traité pratique, par Leclenc et TousSAINT. $3^{\circ}$ édit. 1 vol. in-18, orné de 126 fig. et 2 planches. $\quad 250$

Bétail. - Trailé de l'alimentation du bétail, par E. Duroselle, In-18.

150

mête bovine. - Le type accompli de la bête bovine par A. KraEmer. In-8 orné de 28 fig.

$2 \mathrm{fr}$.

Blé. - Traité sur la vente des grains à la mesure, au poids de l'hectolitre ou au quintal métrique, suivi de tableaux appréciateur's de la valeur des grains suivant la variation de chaque qualité, par HuBaine. In-4. 250 , 
Boeur. - Connaissance générale du bouf, études de zootechnie pra. tique sur les races borines de la France, de l'Algéria, de l'Angleterre, de l'Állemagne, de la Suisse, de la Russie et de la Belgique, par Moln, 1 vol. in $-8^{\circ}$ avec atlas de 83 planches.

$10 \mathrm{fr}$.

Bois. - Traité général de la culture et de 'l'exploitation des bois, par THOMAS, 1840. 2 vol. in- $8^{\circ}$

$10 \mathrm{fr}$.

Isois. - Cubage des bois et tarifs métriques pour cuber les bois carrés ou de charpente. les bois en grime au $5^{\mathrm{e}}$ et au $6^{\mathrm{e}}$ réduit, ainsi que les bois au quart sans déduction, par Gussot. In-8.

$50 \mathrm{c}$.

Boisement et reboisement des montagnes, Landes et terrains incultes. - Traité pratique par Levavasseur. In-8. 125

Holsement et reboisement, des terrains pauvres et même stériles par QUÉHEN-MALLET. 1 vol. in-18. 250

Brasseur. - Méthode pratique pour faire la bière, contenant les meilleures procédés de celte fabrication, suivi d'un traité sur la plantation du houblon, par KoLB, 1832.1 vol. in-!2.

250

Bruyères. Leur défrichement et particulièrement des landes sablonneuses de la Campine, par Phocas Lejeune. 1 vol. in-18. 150

Chênes en taillis à écorces. Semis et culture, par J. Koltz. 1 vol. in-18 orné de 30 figures dans le texte.

Chêne hiège. - Culture, exploitation et aménagement, en France et en Algérie, par A. Rousset, garde général des forêts. In-8 de 80 pages. 250

Cidre. - Trailé élémentaire et pratique de fabr.cation et de conservation; choix et plantation des meilleures variétés de pommiers et de poiriers; eaux-de-vie de cidre, par Hadcheconne, 1 vol. in-8. $2 \mathrm{fr}$.

Cidre $(L e)$, revue mensuelle du polré et du Cidre, organe du Syndicat pomologique de France, publié sous la direction de E. Vimont.

Abonnernent pour la France, $\quad 6$ fr. Abonnement pour l'étranger, $\quad 8 \mathrm{fr}$. Les abonnements datent du $1^{\text {er }}$ janvier de chaque année.

Cidre et Poiré, guide élémentaire et pratique pour la fabrication du Cidre el du Poiré ; la culture du Pommier à cidre, par J. LefÈvre. 1 vol. petit in -18 avec planches. $\quad 150$

Crédit. - Des conditions du crédit appliqué a l'agriculture, par Ch. RIvET, ancien député. In-8. $1 \mathrm{fr}$.

Culture rationnelle. - Simple causerie sur la culture rationnelle agricole, horticole et maraîchère, l'achat et l'emploi des engrais chimiques, par VALLÉE. In 18.

Distillateur pratique. - Traité de distillation indispensable aux propriétaires, cultivateurs, vignerons, bouilleurs de crus, etc; parVIG.NERON. 1 vol. in-18 orné de figures. 2 fr.

Eaux-de-vle de grains et de pommes de cerre. - Instruction théorique et pratique sur leur fabricalion et l'emploi de leurs résidus pour la nourriture des bestiaux, par Mathieu DE Dombasle. 2e édit. In- 8 avec la planche.

$2 \mathrm{fr}$.

Eclosion et élevage artificiels des oiseaux de chasse et de bassecour, par les hydo-incubaleırs et hyulo-mères Roullier el AnNoult. 4 édit. 1 vol. in-18, orné de fig. dans le texte. 1.25 Conomle rurale. - Conrs professé à l'Institut agricole de Hohenheim, par Goeritz, trad. par Rieffel, 1850.2 vol. in-8 ornés de 5 pl. 12 fr. Elaguge des essences forestières.- - Taillis, futaies, baliveaux, etc. a tonte des haies et l'émondage des conifères, par J. VAN Hulle. In-8o, orné de 15 figures. 1 fr.

Engrais. - Ouvrage comprenant l'alimentalion des plantes, les iumiers, engrais des villes, engrais végétaux, engrais azolés et engrais ọhosphatés, par Muntz et GiRard. 3 vol in $-8^{\circ}$, ornés de lig. $18 \mathrm{fr}$.

Engrais chimiques. - Conférence sur la doctrine des engrais chimi- 
ques et l'utilité des champs d'expériences agricoles, par E. Marchand, in-80.

Engrais complémentairog du commerce et de l'industrie (Achal des), par C. FASQUELLE. Brochure in-8.

$1 \mathrm{fr}$.

Étable $\left(L^{\prime}\right)$. - Manuel de zootechnie pratique, par RoBıoU DE LA TRÉHONNAIS. 1 vol. in-18.

$3 \mathrm{fr}$.

Fortune dans l'eau (Une). Comment peut-on faire d'un marais une prairie de bonne nature? Par l'assainissement et le phosphate de chaux, par A. River, secrétaire du comice de Mézières. $2^{e}$ éd., in- $8^{\circ}$.

Fours économiques à círculation al'sir chaud, $p ; r$ A. CASTERmaNN. 1 vol. grand in- 8 avec 5 pl., $2^{\text {e }}$ édit. Bruxelles.

Fronange de Hollande, sa fabrication, par LE SÉNÉCHAL. In-18. 50 c. Fait partie de l'Almanach.de l'Agriculteur praticien pour 1865.

Garances.-Culture, chimie et commerce, par BASTET, 1836,1 v.in-8. 250

Cuauos naturels. - Etude sur les guanos naturels en général et sur le guano du Pérou en particulier, par Crussard. In-8.

$40 \mathrm{c}$.

Gulde des cultivateurs du midi de la France, de la Corse et de l'Algérie, par H. Laure, 1854. 1 vol. in-8.

Haies d'asmément ou de défeuse. Art d'élever le plant de l'épine; de la formation des haies, etc., par Quéhen-Mallet. In-18, 10 fig. 150

Herbages et Prairies Naturelles, par BoltFL. 1 vol. in- ${ }^{\circ}$, orné de 120 fig.

Indigatier. - Art de l'indigotier ou traité des indigofères tinctoriaux. Culture, récolte et fabrication de l'indigo, etc., par Perroteter, 1842. I vol. in-8, orné d'une planche.

$3 \mathrm{fr}$.

Irrigations, comprenant les eaux d'irrigation, les machines, les canaux et les systèmes d'irrigation, par Rovis. 3 vol. in- $8^{\circ}$, ornés de 191 figures.

is fr.

Laiterie (La). - Art de fabriquer le beurre et les principaux fromages français et étrangers, par Pouriau. $4 \mathrm{e}$ édit. 1 vol. in-18 orné de $200 \mathrm{fig} .6 \mathrm{fr}$.

Lapin-Bélier. - Manuel spécial pour leur élevage d'après la méthode suivie avec un plein succès à l'orphelinat a gricole de St-Martin.1 v .in-18. 150

capin donestique (Instruction élémentaire pour élever le). In-18. $50 \mathrm{c}$.

Fait partie de l'almanach de l'Agriculteur praticien pour 1861 .

Lapins - Elevage pratique de toutes les races, par $\mathrm{M}^{\text {ao }}$ la Vsse de BorsLANDRY. In- $8^{\circ}$ de 60 pages, orné de 9 gravures.

Lari et Jambon. - Manuel de la porcherie, par RoBiou de LA TreHONNAIS. 1 vol. in-18.

Lin. Culture et différents modes de rouissage, par DE MOOR. 1 vol. in-18 orné de 14 figures dans le texte.

$75 \mathrm{c}$.

Livre de ln Ferme $(L e)$ et des Maisons de campagne, publié sous la direction de Joigneaux. $4^{\circ}$ édit. 2 vol. grand in-8, ornés de fig. $32 \mathrm{fr}$.

uois naturelles de l'agrieulture, par LiEBIG. 2 vol. in- $8^{\circ}$. $10 \mathrm{fr}$.

Iupin. Sa culture et ses usages, par Koltz. 1 vol. in-18. $1 \mathrm{fr}$.

minion rustlque des Dames, par Mme Millet-Robinet. 13e édit. 2 vol. in-18, ornés de 269 grav.

Mrison rustique du Xxe siècle, publiée sous la direction de MM. Bailly, BıXı0 et Malepeyre. 5 vol. gr. in-8 ornés de 2,500,gr. 3950

Maitage pracique, par Chatelain et Vollier, $3^{e}$ édit. 1862. In-8. 2 fr.

Médoc. - Observations sur la culture de cette contrée, suivies de la manière de soigner les vins, par Joubert. 1851. In-8. 250

Meumerie francaise $(\mathrm{La})$ et les procédés nouveaux appliqués par la meunerie étrangère, par KREMER. In-8 avec $5 \mathrm{pl}$.

$4 \mathrm{fr}$.

Moudre $(A r t d e)$, ou mémoire sur les moyens employés pour empêcher que la chaleur produite par la pression et le frottement des meules soit préjudiciable à la farine, par Van Lerberghe. 1849. In-8

Mîrler et vers à sole. Production, industrie, commerce de la soie, par A. Gobin. 1 vol. in-18 de 270 pages, orné de 36 fig. 


\section{$-7-$}

Oisfaux de basse-cour (Traité des), d'agrément et de produit, par A. Gobin, 2e édit. 1 vol. in-18 orné de 93 fig. 350 Dlivier. - Culture et produits par Raibaud-Lange. In-8 de $92 \mathrm{pag} .2 \mathrm{fr}$. pôturage et alimentation à l'étable, et utilisation des fourrages obtenus par différents modes de préparation, par Weiske. In-18. $75 \mathrm{c}$. Pllloxera. - - Destruction infaillible, disparition forcée par l'antiphylloxerique, etc., par L. Chassignolle. Brochure in- $8^{\circ} . \quad 1 \mathrm{fr}$. Plantes Vénéneuses, considérées au point de vue de l'empoisonnement des animaux de la ferme, par Connevin. 1 vol. in $-8^{\circ}$ avec $f i g$. $6 \mathrm{fr}$. plgeons de volière, de colombier, messagers, etc., par Gobin. 1 vol. in-18 orné de 46 fig. $\quad 3$ fr. pisciculture. - Etudes historiques et pratiques, par le vicomte $\mathrm{H}$. DE Beaumont. 1 vol. in-18, avec fig. dans le texte. 350 Pisciculture pratique. - Traité des procédés de multiplication naturelle et artificielle des poissons, par Fraiche. 1 vol. orné de 9 fig. $2 \mathrm{fr}$. Pomme te terre. Leur culture dans les champs, les jardins, etc., par QuÉHeN-Mallet. $3^{e}$ édition, 1 vol. in-18. 15 ( ) Pomme en Normandie. Culture et fabrication du cidre, par LESUEUR. In-18.

$75 \mathrm{c}$.

Pommier cidre, sa culture; plantation et ébranchage des arbres à haute futaie, par LaCallLE. 1 vol, in-18 cart. 150 Pomm fer et Cidre. - Guide pratique pour la culture du Pommier et la fabrication du cidre, par Brassart. $8^{\circ}$ édition, 1 vol. in-1S. $\quad 250$ Poulaller $(L e)$. - Monographie des poules indigènes et exotiques, par Ch. JACQUE. 2e édit. 1 vol. in-18, orné de 117 grav. 350 Poulaillers (Les) de Biamonit, par P. Revir. 1 vol. in- $8^{\circ}$ orné de 150 figures inédites. - Prix : $5 \mathrm{fr}$.: franco 550 Prairies (Notes sur les), par C. Fasquelle. Brochure in-8. $50 \mathrm{c}$. Pratique avec science, par RoBiou dE LA TRÉHonNals. I v.in-18. $2 \mathrm{fr}$. meboisement (Traité du), ou manuel du planteur, par H. Bazelaire. 2 e édit. 1 vol. in-18.

Reproduction en zootechnie. - Croisement, sélection, métissage. par BARON. 1 vol. in- $8^{\circ}$, orné de 56 fig.

Reuche $(L a)$. - Méthode nouvelle essentiellement pratique, par A. Vi-

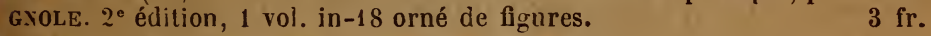

Féche de Cueuvres. - Petit traitá d'apiculture pratique pour la conduite des ruches à cadres mobiles, par Deciry. $2^{\mathrm{e}}$ éd., in-18, $28 \mathrm{fig}$. 110 Rucher. - Vingt ans auprès d'un rucher, ou cours d'apiculture rationnelle en 8 leçons, purement pratique et mise à la portée de tous, par un apiphile. 1 vol. in-18.

150

sel. Son emploi démontré par la pratique, à l'usage des cultivateurs et des fermiers. In-So. $60 \mathrm{c}$.

ฐorgho (Composition chimique et extruction du sucre de la canne de), par Paul Madinier. In-8. $\quad 60 \mathrm{c}$.

Eorgho suere $(L e)$, sa culture comme plante fourragère et comme plante alcoolisable et saccharine, par Louis HERvé. In-8. $\quad 60$ c.

Taupler ( $L^{\prime}$ art $d u$ ), ou Méthode amusante et infaillible pour prendre les taupes, par Dralet. 17 e édit. 1 vol. in-12, fig. 150

(Cocon. - Nouvelles études sur le cocon. 1856. In-8. $\quad 1 \mathrm{fr}$.

5 Cocons. - Procédé pour le battage des cocons, ou moyen d'obtenir des cocons le plus de soie possible. 1843. In-8. 150

Magnaneries. - Expériences sur la ventilation des magnaneries. 1831. 1 vol. in-8 avec pl. $\quad 3 \mathrm{fr}$. Mûriers. - Quatre mémoires sur le mûrier. 1840-43. In-8. 150 sole. - Mémoire sur la filature. 1839.1 vol. in -8 avec $7 \mathrm{pl} . \quad 450$ vers à sole. - De l'Influence des phénomènes météorologiques sur les éducations des vers à soie. 1850. In-8. 
VIgne. - Résumé des opérations à suivre pendant le cours de la végétation de la vigne et Etude de la rupture des bourgeons à l'état herbacé, par E. Troullet. 2e édit. Tableau in-folio, fig. et texte.

$60 \mathrm{c.}$

Vigme. - Culture de la vigne et fabrication du vin dans la Moselle, par DUFOUR. 1851. 1 vol. in-18.

Vigne. - Manuel pratique du Vigneron comprenant un abrégé sur la culture de la Vigne, ses maladies et les maladies des viris, par J. Bonjour. In-18, orné de fig. et de planches.

$1 \mathrm{fr}$.

vigne $(\boldsymbol{L} a)$. et le Phylloxern. Culture de la vigne pour la préserver de la décrépitude et du Phylloxera, par Quéhen-Mallet. In-18. $1 \mathrm{fr}$.

\section{Ouvrages de M. Georges Ville.}

La production végétale et Ies engrais ehimiques (conférences de Vincennes), 3e édit. $1890.1 \mathrm{v}$. grand in- ${ }^{\circ}$, avec fig. et tableaux. $8 \mathrm{fr}$.

Le propriétaire devant sa ferme délaissée (conférences de Bruxelles). $4^{\circ}$ édition. 1890. 1 vol. in-18.

$2 \mathrm{fr}$.

Les engrais chimiques (conférences de Bruxelles), $2^{\mathrm{e}}$ édition. 1890. 1 vol. in-18.

$2 \mathrm{fr}$.

Les engrais chimiques. - Entretiens agricoles donnés au champ d'expériences de Vincennes.

1. Les principes et la théorie. 1890. 1 vol.in-18, av. tableaux. $3 \mathrm{fr} .50$

II. Les culture 3 spéciales. 1890.1 vol. in -18 , avec tableaux. $3 \mathrm{fr} .50$

III. Le fumier et le bétail. 1890.1 vol. in-18. $3 \mathrm{fr} .50$

École des Engrais chimiques, premières notions de l'emploi des agents de fertilité. $6^{\text {e }}$ édit. 1 vol. in- 18 .

$1 \mathrm{fr}$.

In. - Art de faire le vin. - Influence du climat et du sol sur le raisin; de la vendange; de la fermentation, de l'alcool ; de la coloration; du décuvage, des maladies, etc., par Chaptal; 3• édit. revue par de Valcourt. 1839. 1 vol. in-8 avec $p l$.

$6 \mathrm{fr}$.

Vinification. - Nouvelle méthode de culture de la vigne et fermentation vineuse, suivi de l'art de faire le meilleur vin possible par un procédé nouveau, par Aubergier. 1825. 1 vol. in-12.

Vims. - Traité pratique, par MachaRd. 5e édit. 1 vol.in-18. 350

\section{Bibliothèque de l'Horticulteur praticien.}

Encouragée par MM. les Ministres de l'Agriculture et du Commerce et de l'Instrueticn publique.

Ade-roémoíre pour les semis et travaux divers à faire pendant les douze mois de l'année dans le Jardin fleurisıe, par A. Goin. 1 vol. in-18 (Sous presse).

Aide-mémoire pour les semis et travaux divers à faire pendant les douze mois de l'année dans le Jardin potager, par A. GolN. I vol. in-18 (Sous presse).

A Imanach du Jardinier fleuriste, suivi le notes sur le jardin potager. 1 vol. in-18 avec fig. dans le texte. Les arnées 1861, 1863, 1868, 1869 , 1871-72, 1873 seules sont disponibles, chaqui.

$50 \mathrm{c}$.

Anumas a fruit comestible. - Culture actuelle comparée à l'ancienne culture, suivie d'une notice sur la culture forcée du fraisier, par Gontier. 1 vol. in-32, orné de $13 \mathrm{fg}$. dans le texte et hors texte. $3 \mathrm{fr}$.

Arborlculture. - Manuel pratique renfermant ce que les meilleurs auteurs et les praticiens ont dit de mieux sur le défoncement, la plantation. les formes, la taille et la mise à fruil des arbres fruitiers, par l'abbé Raoul, (Nouvelle édition en préparation.)

A pprouvé pür la Commission des bibliothèques scolaires.

Arborlculture des Ecoles primaires ou Notions d'arboriculture 
fruitière mises à la portée des enfants, par J. BRÉmond, $3^{\epsilon}$ éd. $2^{\mathrm{e}}$ lirage.

1 vol. in-18 el atlas de 107 fig.

Approuvé par la Cummission des bibliothèques scolaires.

$2 \mathrm{fr}$.

Arbres fruitiers. - Conseils sur le choix, la culture et la taille, pouvant convenir aux provinces du nord, de l'est, de l'ouest et du centre de la France, par le comte DE LAMBERTye. In-18, orné de $33 \mathrm{fig}$. (Nouvelle edition en préparation).

Approuvé par la Commission des bibliothèques scolaires.

Artichants et cardons. Leur culture, par un horticulteur. In-18, avec fig. (Sous presse).

1 vol. in-18, orné de 11 gravures dans le texte, et d'un plan d'asperges.

Asperges (Semis, plantation el culture des). Méthode d'Argenteuil, etc., par Bossin, $4^{\circ}$ édit., revue et corrigée par l'éditeur, 1 vol. in -18 2vec fig. $1 \mathrm{fr}$.

Asperges. - Culture ordinaire et forcée; semis, plantation el cueillette, par Levormand. I vol. in-18, orné de 11 gravures dans le texte, et d'un plan d'aspergerie.

$1 \mathrm{fr}$.

Botaniste et herboriste (Petit Manuel du), donnant la description de 220 plantes officinales, suivi de principes de médecine, de pharmacie, d'hygiène et d'économie domestique, etc., par L. T., F. M. et P. M., $3^{\ominus}$ édit., revue et corrigée par l'éditeur, 1 vol. in-18 orné de 80 fig. 250

Bonturer, greffer, marcotter et semer (Guide pour) les plantes d'ornement, annuelles ou vivaces, arbres et arbustes, extrait en partie du Jardin fleuriste, $2^{\circ}$ éd.. complétée par l'éditeur. In-18 orné de 35 fig. 1 fr.

Cactées. - Leur culture, suivie d'une description des principales espèces el variétés, par Palmer. 1 vol. in-18, orné de $33 \mathrm{fig}$. $2 \mathrm{fr}$.

Canna. - Histoire, culture et multiplication, par CHATÉ. 1 vol. in-32, orné d'une fig. hors lexte. 150

Champignon. - Sa culture en plein air, dans les caves et dans les carrières, par Laızier. 1 vol. in-18, orné de 7 fig. $60 \mathrm{c}$.

Champignons (Culture des), avec l'indication d'une nouvelle méthode pour en obtenir en tous lieux par l'emploi de la mousse, par SaLLE, $5^{\circ}$ édit., revue et complétée par l'éditeur. 1 vol. in-18, orné de $20 \mathrm{fig}$. $1 \mathrm{fr}$.

Champignon comestible. - Instructions pratiques sur sa culture, par JACQuin aîné. $\ln -18$ de 24 pages. $60 \mathrm{c}$.

Champignoms comestibles et vénéneux de France. - Guide pour les reconnaître, par ElofFe (KRoenishFranck). 1 vol. in-32, orné de 11 planches donnant la figure de 114 champignons coloriés. $\quad 5 \mathrm{fr}$.

Chrysanthème. - Culture et multiplication par un amateur, revue par M. НARIOT. 1 vol. in-18. (En préparation.)

Cinérsires. - Cultire et multiplication, par Chaté. 1 vol. in-32, orné d'une figure hors texte. $50 \mathrm{c}$.

Conifères de petites et grandes dimensions. - Culture ornementale et forestière, par MORLET. 1 vol. in-18. 350

Culture marnîchère. - Traité théorique et pratique de culture maraîchère, par Rodigas, $3^{\circ}$ édit. 1 vol. in-18. 350

Cyclamen. Culture et multiplication par un amateur. In-18. $50 \mathrm{c}$.

Fleurs de pieine terre et ae fenêtres. - Conseils sur leur culture, pouvant convenir au nord, à l'est, à l'ouest et au centre de la France, par le comte de Lambertye. $2^{e}$ éd. in-18.

Approuvé par la Commission des bibliothèques scolaires.

Fraisfer. - Culture en pleine terre suivie d'un choix des meilleures variétés à cultiver, par le comte de LaMBertye. 1 vol. in-18. $1 \mathrm{fr}$. Approuvé par la Commission des bibliothèques scolaires.

Fraisier. - Culture forcée par le thermosiphon, par le comte DE LAMBERTYE. 1 vol. in-18, fig.

125

Fuchsla. - Histoire et culture, suivies de la description de 540 espèces et variétés, par F. Porcher, $4^{\circ}$ édit. 1 vol. in- 18.

$2 \mathrm{fr}$.

Géranium et élaruonium. - Multiplication et culture, par MALET et VERLOT. (Nouvelle édition en préparation.) 
Craminées. - Choix et culture des graminées propres à l'ensemencement des pelonses et des prairies, par Courtors-Gérard. 1 vol. in-32, orné de 19 fig. hors texte.

Jardin meuriste $(L e)$. - Instruction pour la culture des plantes annuelles, bisannuelles, vivaces; plantes à feuilles ornementales; oignons à fleurs; arbres et arbustes, par Lemaire, Lequien, Bossin, Bernarnin, Carriére, comte du Buysson, Palmer, Porcher, Riviére père et fils, etc., revue et complétée par l'éditeur. $5^{\text {e }}$ éd. 1 vol. in-18, orné de 250 fig. 350

Jardinage. - Éléments de jardinage pouvant convenir aux provinces du nord, de l'est, de l'ouest et du centre de la France, par le comte DE LAMBERTYE. 1 vol. in-18 aver fig. dans le texte.

Approuvé par la Commission des bibliothèques scolaires.

Jardinier lllustré (Le Nouveau) pour $1893 .-$ Ouvrage pratique pour la culture et la taille des arbres fruitiers; la culture en pleine terre et forcée des légumes; des plantes depleine terre. de serre froide et tempérée, de serre chaude, par MM. HérincQ, Lavallée, Neumann, Verlot, Courtols-Gérard, Pavard, Cels et Burel, revu et corrigé par l'éditeur. $29^{\text {e }}$ éd. 1 vol. in-18 de 1,760 pages, or'né de 580 figures dans le texte. $7 \mathrm{fr}$.

La $1^{\text {re }}$ édition de cet ouvrage a paru en 1865.

Approuvé par la Commission des bibliothèques scolaires.

Lantanas. - Culture et multiplication, par Chaté: 1 vol. in-32, 50 c.

Légumes. - Conseils sur les semis de graines de légumes, offerts aux habitants de la campagne, par le comte DE LAMBERTYE, $4^{\circ}$ éd. In-18. $1 \mathrm{fr}$. Approuvé par la Commission des bibliothèques scolaires.

régumes. - Conseils sur les semis et la culture des légumes en pleine terre, offerts aux habitants des départements du Rhône, de l'Ain, de la Loire et de Saône-et-Loire, par le comte DE LAmberTye. 1 vol. in-18.

$1 \mathrm{fr}$.

Légumes et neurs. - Conseils sur la culture de légumes et de fleurs sous un, deux ou trois châssis, pendant les douze mois de l'année, par le comte DE LAMBERTYE. 1 vol. in-1 8 orné de fig.

Approuvé par la Commission des bibliothèques scolaires.

$1 \mathrm{fr}$.

Melon. - Cultare simple et précise par laquelle on obtient des melons d'une grosseur extraordinaire. d'une qualité et d'un goût exquis, par DuFour de Villerose. 4e éd. complélée par l'éditeur. In-18, $23 \mathrm{fig.} 1 \mathrm{fr}$. Approuvé par la Commission des bibliothèques scolaires.

Mclon : Concombre vert long; Concombre cornichon; Courge à la moelle et Potiron vert d'Espagne. - Conseils sur leur culture à l'air libre, par le comte dE Lambertye. 1 vol. in-18 orné de $18 \mathrm{fg}$.

Approuvé par la Commission des bibliothèques scolaires.

Melon. - Instructions pratiques sur sa culture sous châssis, sous cloche et en pleine terre, par Martin JacQuin. In-18 de 36 pages. $\quad 60 \mathrm{c.}$

Irelon et Concombre. - Culture forcée par le thermosiphon, par le comte dE LAMBERTYE. In- $8^{\circ}$, fig.

125

abillet. - Culture, etc. 1 vol. in-18 avec fig. (En préparation).

Palmiers (Les) de plein air de la France, par J.-B. Chabaud, jardinierbotaniste. 1 vol. in-18, orné de 15 fig. dans le texte. (En préparation.)

Pêcher. - La direction des arbres par le pincement des feuilles, et notamment du pêcher, par Grin, $3^{e}$ édit. 1 vol. in-18, orné de 6 fig. $1 \mathrm{fr}$.

pêcher en espalier. - Culture, plantation, taille el direction, démontrées par 125 figures dessinées el accompagnées d'un texte descriptif, par E. Couturıer, ancien arboriculteur à Montreuil, etc. 1 vol. in- $8^{\circ} .5 \mathrm{fr}$. Phlox. - Culture et multiplication, par Lierval. 1 vol. in-32, orné de 5 fig. hors texte.

$1 \mathrm{fr}$.

Plantes nquatiques. - Multiplication et cullure, par HÉLYE. 1 vol. $\mathrm{n}-32$, orné de 16 grav. dans le texte et hors texte.

150

Plantes it feublles ornementales en pleine terre (Les): Caladium, Canna, Gynerium, Musa, Solanum, Wigandia, etc. Bolanique et culture, par le comte de Lambertye. (Nouvelle édition en préparation). 
Plantes de pleine terre, annuelles, bisannuellew et vivaces, (Culture des), par MaRTIN JACQUiN. 1 vol. in-18 de 100 pages. 150

Plantes molles de pleine terre: Géranium, Heliotrope, Pensée, Pélunia, Verveine. Culture pratique par F. Du Buysson. In-18, fig. $1 \mathrm{fr}$.

Fommes de terre. - Choix, culture ordinaire et forcée ; culture hivernale; récolte et conservation, par Courtoss-GÉRARD. 1 vol. in-32. (En préparation).

i Keine-Marguerite (Culture de la), par MaLingre. In-18. $\quad 40 \mathrm{c}$.

Resier. - Culture, multiplication et taille, par un amateur. 1 vol. n-18, orné de 38 fig. dans le texte. $\quad 150$

Resier.- Culture forcée, suivie de la culture en pots et en plein air, et de la culture forcée du Lilas, par un horticulteur. In-18. $50 \mathrm{c}$.

Eosier. - Semis, culture et taille, suivis de la Taille des Arbustes d'agrément de pleine terre et de l'Oranger, par FoRNeY, $4^{\ominus}$ éd. 1 vol. in-18, 50 fig.

$2 \mathrm{fr}$.

reosièriste. - Aide-mémoire pour les soins à donner aux rosiers forcés et de pleine terre pendant toute l'année. in-18. $50 \mathrm{c}$.

Temate et Iraricot. - Culture forcée par le thermosiphon, par le comte DE LAMBERTYE. In- $8^{\circ}$, fig.

125

verveimes.- Culture et multiplication, par Chaté. 1 vol. in-32. 50 c.

Vigne.-Culture forcée, parle comte DE LAMBERTYE. In-8 ${ }^{\circ}$, fig. 125

\section{Arbres fruitiers, Botanique, Culture potagère, Jardinage.}

Amanach Gressent pour 1893, contenant les principes élémentaires d'Arboriculture et de Potager, par Gressent. In-18. $50 \mathrm{c}$.

Les années $1867,1869,1870,1876,1878,1879,1881,1883,1884$ et 1886, chaque :

$75 \mathrm{c}$.

Arboriculture ( $L$ ') fruitière comprenant la culture intensive et exiensive des fruits de table, etc., par Gressent, $9^{e}$ édit. 1 vol. in-18 avec 430 fig. dans le texte.

$7 \mathrm{fr}$

Arboriculture fruitière. - Cours pratique, par Delaville, $3^{e}$ édit.

1 vol. in-8, orné de 284 fig.

$6 \mathrm{fr}$.

a rboriculture fruitière.- Cours pratique par le frère HenRi $2^{e}$ éd.

1 vol. in-8 $8^{\circ}$ orné de 197 fig. et 2 pl.

$5 \mathrm{fr}$.

A rbres al'agrément. - Traité de la taille des grands arbres plantés en bordure des chemins, sur les places publiques, allées d'avenues, massifs, suivi de celle de l'amandier, du noyer et du châtaignier, par J. GaUTIER, 1 vol. in-8, orné de $18 \mathrm{fig}$.

$2 \mathrm{fr}$.

Arbres fruitiers Conseils sur le choix et la forme des hrbres avant la plantation, suivis d'un traité sur leur culture et leur restauration, par l'abbé Lefìvre. $7^{\mathrm{e}}$ édition. $1 \mathrm{vol}$. in-1S orné de planches. $2 \mathrm{fr}$.

arbres et arbrisseaux à fruits le table. $6^{\circ}$ édit. du Cours d'arboriculture par DUBREuIL. 2 vol. in-18 ornés de $748 \mathrm{fig} . \quad 1150$

Arbres fruitiers (Instruction élémentaire sur la conduite des), par Dubreuil, 9 édit. 1 vol. in-18, fig. 250

arbres fruitiers. - Instructions pratiques sur la taille et la conduite des arbres fruitiers, par ChaROLLOIS. In-8 avec pl.

150

arbres fruitiers. Manuel populaire de culture, marcottage, bouturage. greffage et taille, par P. JolgneaUx. 1 vol. in-18 orné de 111 tig. 250

a rbres fruitiers (Taille raisonnée des), par J. Hardr, ge édit. 1 vol. in-8 avec 134 figures.

550

Arehitecte paysagiste $\left(L^{\prime}\right)$. Théorie et pratique de la création des Parcs et Jardins; - Cours d'aquarelle en quatre lecons, accompagné de 4 chromo-lithographies; - Notions usuelles de droit; - Comptabilité des travaux, par A. PÉAN. 1 vol. in-8o, orné de $132 \mathrm{fig.} 20 \mathrm{fr}$. ssperges. - Culture industrielle et hivernale, suivies de la manière d'en faire des conserres, par P. Ronceray. In-8. 
sperge. - Culture à la charrue. Résullat obtenu dans un sol de médiocre qualité, bénéfice net, 6,000 fr. à l'hect., par A Vauvel. $2^{\circ}$ édit. 1 vot. in-18.

$1 \mathrm{fr}$.

Asperges d'A rgenteull. - Instructions générales sur leur culture, par L. LHĖRault. In-18 de 48 pages.

$1 \mathrm{fr}$.

Botanique des lemolselles, par Boltard, 1 rol. in-8, orné de $64 \mathrm{pl}$. contenant $1096 \mathrm{fig}$. noires représentant les caractères généraux et pécifiques des plantes.

$6 \mathrm{fr}$.

Botanique populaire contenant l'histoire complète de toutes les parties des plantes, etc., par H. LECOQ. 1 vol. in-18 orné de 215 fig. 350

Botanique. - Traité général de botanique descriptive et analytique, par Lemaout et Decaisne, $2^{\circ}$ édit., 1 fort vol. in-4 $4^{\circ}$ orné de $5500 \mathrm{fig} .30 \mathrm{fr}$.

Champignons supérieurs du Tarn, par J. BEL. 1 vol. in- $8^{\circ}$ orné de 32 planclies coloriées.

8 fr.

Chasselas. - Traité pratique de la culture du chasselas à Thomery par J. Bureau. $2^{e}$ édit. augmentée d'un chapitre sur les maladies de la vigne. In-8 de 30 pages orné de 3 figures. 150

Chrysanthème, sa culture, par Cuvelier. 1 vol. orné de 10 fig. 150 Chrysanthèmes (Les). - Description, culture, emploi, par C. Bellair et V. Bérat. 1 vol. in -18 orné de 21 figures. $2 \mathrm{fr}$.

Chrysanthèmes d'hiver. Liste descriplive, par G. de Meulenaere. In-18 oblong, cart.

150

Culture marnichère. Traité pratique, par le frère Henrı. 1 vol. in-18 orné de 2 planches.

$4 \mathrm{fr}$.

Culture maraîchère à sol couvert, par J. NANOT. In- $8^{\circ} . \quad 50 \mathrm{c}$.

Culture potagère à la portée de tous, par Burvenich. 2。 édit., 1 vol. in-18 orné de figures.

Cypripedium (Les) et genres affines, par Angiolo Pucci. 1 vol. in-18.

250

Cypripédium, Sélénipédîum et Tropédium, (Magnographie des), comprenant la description de toutes les espèce, variétés et hybrides existant jusqu'à ce jour, par DEsBors. 1 vol in-18 orné de 7 fig. 150

Figuler blane d'A rgenteuil. - Sa culture, par L. LHÉrault. In-18 de 24 pages.

$50 \mathrm{c.}$

Fleurs. - Grand assortiment de planches coloriées, à 20, 30 et 40 c. la planche, au choix.

Fleurs de plelne terre (Les). Description et culture, par-ViLmorin Andrieux, $3^{e}$ éd. 1 fort vol. petil in-8 avec supplément orné de près de $1,400 \mathrm{gr}$. $16 \mathrm{fr}$.

Flore ale In côte-d Or ou description des plantes indigènes, cultivées et acclimatées dans ce département, par LOREY et DURET, $1831.2 \mathrm{v}$.in- $8^{\circ} .8 \mathrm{fr}$.

Fougères de serre et de pleine terre. - Traité théorique el pratique pour leur culture, par J. Wolff. I vol. in-18. 125

Frafaler. Sa culture commerciale, par Mulie. ln-18 orné de fig. $1 \mathrm{fr}$. Fruits. - Grand assortiment de planches coloriées à 25 et 50 c. la planche, au choix.

Fruits. - Traité complet des fruits de toute espèce, comprenant la description des fruils tant indigènes qu'exotiques, leur emploi dans l'alimentation, l'économie domestique, la médecine et les arts, leur conservation par les divers procédés conmus; leur amélioration par l'influence des engrais; des semis, de la taille des arbres, de l'incision annulaire, etc., par Couverchel. 1852. I vol. in-8;

750

Frulta de choix. Description, culture et commerce des variétés les plus intéressantes à cultiver dans les vergers et les jardins, par G. Michels. $2^{\circ}$ édition, 1 vol. in-18 orné de 35 figures dans le lexte, accompagné d'un tableau composé de 47 fruits divers de grandeur naturelle et coloriés. - Prix : 3 fr. 50 ; franco $4 \mathrm{fr}$,

Mortleuiteur français $\left(L^{\prime}\right)$. - Journal des amaleurs et des intérêts 
horlicoles, publié sous la direction de F. HÉrincQ, depuis l'annee 1850 jusqu'à la fin de 1872. 21 volumes in-8, accompagnés de 450 planches col. environ. Au lieu de $210 \mathrm{fr}$. $150 \mathrm{fr}$.

Il ne reste plus que 8 collections complètes de disponibles. Les volumes séparés 7 fr., franco.

Jardins. - Manuel de l'amateur des jardins. Traité général d'horticulture, par Decaisne Naudin. 4 vol. in-8, ornés de $537 \mathrm{fig}$. $30 \mathrm{fr}$.

Jardins (Traité de la composition et de l'ornement des), avec $161 \mathrm{pl}$. représentant, en plus de $600 \mathrm{fig}$, des plans de jardins, des machines pour élever les eaux, etc., 6 e édit. 2 vol. in-4, oblong. $\quad 25 \mathrm{fr}$.

Jardins d'agrénient. Tracé et ornementation, par Bona. $4^{\mathrm{e}}$ édit. 1 vol. in-1 8 orné de 258 figures.

250

Iardin frultter. - Le nouveau Jardin fruitier, dédié aux jeunes cultivateurs, par lihevalier. In-18, orné de fig.

$1 \mathrm{fr}$.

Jardins frultiers et potagers (Instructions pour les), par DE LA QUINTINYE, extrailes de l'édition publiée en 1697, et accompagnées de notes sur les nouveaux modes de culture, de forme et de conduite d'arbres fruitiers. 1 vol. in-8, orné de fig.

350

Drehidées. - Trailé théorique et pratique sur la culture des orchidées par le comte F. Du Buysson. 1 vol. in-8.

$6 \mathrm{fr}$. Le manuscrit de cet ouvrage a été couronné en 1875, à l'Expos. intern. de Cologne. Drehidées. Structure, histuire et culture, par LEwis Castle. 1 vol. in-18 cart. fig.

$3 \mathrm{fr}$.

Pares et jardins. - Prix de règlement ou tarif des travaux de jardinage, de plantations, d'exploitation des forêts, etc., par LEcoQ, architecte de pares, 1859 . In- $8^{\circ}$.

$3 \mathrm{fr}$.

Pares et Jardins. - série de prix applicable aux travaux de Parcs et jardins, établie par la Chambre syndicale des architectes-paysagistes, entrepreneurs de jardins et horticulteurs de France. $1^{\text {re éd } .1881 .1 ~ v . ~ i n-4 . ~} 5$ fr.

Pares et Jardins. - Traité complet de la création des parcs et des jardins pysagcrs, de la culture des arbres d'ornements, etc. par GressEnT. $4^{\circ}$ édit. 1 vol. in-18, orné de $291 \mathrm{fig}$, dans le texte.

$7 \mathrm{fr}$. Patates, leur culture à la portée de tout le monde, par M. Jourdan. $\ln -8^{\circ}$.

$40 \mathrm{c}$.

Plantes putagères. - Description et culture par VILMORIN-ANDRIEUX. 1 vol. in- 8 avec de nombreuses figures.

$12 \mathrm{fr}$.

Plantes de serre. - Traité théorique et pratique de la culture de toutes les plantes qui demandent un abri, par DE PUYDT. 1 vol. in-8. $6 \mathrm{fr}$. plantes de serre froide, d'appartements, de jardins d'été, par DE PUydT. $3^{\circ}$ édit. 1 vol. in- 18.

Poiriers les plus précieux, parmi ceux qui peuvent être cultivés à haute tige aux vergers et aux champs, avec les figures au trait des fruits, par J. DE LIRON D'AIROLLES. 1874.9e édit. In-8. $2 \mathrm{fr}$. Poires.-Quarante Poires divisées en 4 séries de 10 poires, dont la maturation a lieu pendant les mois de juillet à mai, contenant le nom, la synonyme, la description des poires, de l'arbre, le mode de culture; l'époque de la cueillette du fruit arec la silhouette de chacun et de grandeur naturelle, suivies de considérations sur la culture et la taille du poirier, par P.DE MoRTILlet. $3^{\circ}$ édit. in-8.

350

Poires d'ellte. 50 variétés pour grande et petite culture. Descriptions et culture, accompagnées de 50 figures noires dessinées d'après nature et de 37 figures dans le texte, par G. Michels, horticulteur. 1 vol. in-18, - Prix : 2 fr. 56; franco 275 Poirler et Ponmier; - Traitement de la branche à fruit, par l'abbé LEFÈVRE. In-18, avec $2 \mathrm{pl}$. $60 \mathrm{c}$.

Potager $(L e)$ et la maîtresse de maison. - Tableaux indiquant les quantités de chaque légume nécessaire pour un ménage ; les quantités de terrain et celles de semences correspondantes; les époques de chaque ensemencement et celle des récoltes, par un amateur. Brochure in- $8^{\circ}$ oblong. $1 \mathrm{fr}$. 
Pomologie - Dictionnaire de pommlofle, cuntenant l'histoire, la description, la culture, la fertilité, la maturité, la qualité et la silhouetle de grandeur naturelle des fruits anciens et modernes les plus généralement connus et cultivés, par André LERor, pépiniériste, 6 vol. gr. in-8. $30 \mathrm{fr}$.

Les Pulres, 915 variétés. 2 vol. - Les pommes, 527 variétés. 2 vol. - Les Abricots, 34 variétés, et Les cerises, 127 variélés. 1 vol. Le Pêcher, 143 variétés, 1 vol.

Tors les volumes se vendent séparément $5 \mathrm{fr}$.

Potager faclle (Le), par H. Boulmé, 1 vol.in-18 de 188 pages, 250

Potager moderne ( $L e)$. Traité complet de la culture des légumes, par Gressent, 8e édit. 1 vol in-1S avec 131 fig. dans le texte. $7 \mathrm{fr}$. Ouvrage couronné par la Société centrale d'horticulture de Paris.

Primeveres de chine. L'art de les cultiver, par H. Pascal, jardi. nier chef. Broehure in- $8^{\circ}$ de 16 pages.

Fochers. - Considérations générales sur les différents genres de rochers artificiels, sur leur nature, sur leur mode de construction, etc., par Combaz, architecte paysagiste. In-8 ${ }^{\circ}$ orné de 7 grandes tigures. 1 fr.

Hoses - Dictionnaire des Foses, ou guide général du rosiériste, contenant la description détaillée de plus de 6,000 rosiers, tant anciens que modernes, par MaX Singer. 2 forts vol. in-18 avec fig.

$12 \mathrm{fr}$.

teoses. - Nouvelle classificalion, par F. CRÉPIn. Brochure in- $8 . \quad 60 \mathrm{c}$.

zoses. - Journal des Roses, fondé par MM. S. Cochet et C. BerNARDIN. - Ce Journal parait tous les mois. Les livraisons sont composées de 16 pages de texte et d'une planche coloriée.

Prix de L'abonnement pour l'année : France, $12 \mathrm{fr}$; - Europe, $13 \mathrm{fr}$. 20; - Asie, Afrique, Amérique et OCÉanie, $14 \mathrm{fr}$.

Livraisons séparées, 1 fr. 25. - Pas de numéro spécimen.

Les années $1887,1888,1889,1890,1891$ et 1892 , brochées, sont disponibles chaque année.

$15 \mathrm{fr}$.

Truffe. - Etudes sur les truffes comestibles au point de vue botanique entomologique, forestier et commercial. par H. BonNET. In-8. $2 \mathrm{fr}$. Mémoire couronné par l'Académie des sciences.

Végetution. - Recherches chimiques sur la végétation, par de SAUSSURE. 1804. In-80 avec pl. et tableaux.

5 fr.

Vignne en treille, sa culture, par l'abbé Lefk̀vre. In-18, 1 pl. $30 \mathrm{c}$.

vigne et arbres fruitiers. Nouveau mode de culture, par J. DesBols. 1 vol. in $-8^{\circ}$.

350

\section{Bibliothèque du Sportsman}

Alouette - De la Chasse de l'alouette au miroir avec le fusil, par NéRÉe-QUÉPAT. 1 vol. in-18 orné de grav.

150

Bécasse. - Le chasseur à la bécasse par Polet de Faveaux (Srlvain) 1 vol. in-18 orné de 35 fig. humoristiques dans le texte, dessinées par Ý́LiGien Rops. (Nouvelle édit. en préparation.)

Chasse. (Los paramientos de la Caza), ou règlements sur la chasse, par Don Sancho LE Sage, roi de Navarre, publié en 1180 Nouvelle éd. avec introduction et notes du trad. 1 vol. in-18. $2 \mathrm{fr}$.

Chasse. - Soixante années de chasse. - Pratique de la chasse au chien courant à pied et à cheval; au chien d'arrêt en plaine et sous bois, au marais, sur les étangs et sur les rivières, par J.-A. CLAMART, 3e édit. revue et corrigée par l'éditeur. 1 vol. in-18 orné de 60 fig. dans le texte.

350

Chasse à courre et à tirr. - Nouveau traité par A. DE LA RuE et le marquis de Chenville. 2 vol. in-8 ornés de 141 fig. dans le texte par Ch. JACQUE, LANÇON, etc., et accompagné de 41 fanfares.

$20 \mathrm{fr}$.

Le même, imprimé sur papier vergé.

$40 \mathrm{fr}$.

Chasse aux petits olseaux, par Crahay, 2 éd. In-18, fig. 150

Chasseur infuluuble. - Chasse au chien d'arrêt, par Marksman, trad. de l'anglais sur la $3^{\text {e }}$ édit., augmenté d'un appendice sur la Chasse 


\section{$-13-$}

des oiseaux de marais. Nouvelle édit., revue et corrigée par l'éditeur. 1 vol. in-18, orné de 31 fig. 350 Cheval domestique (Les Origines du). d'après la paléontologie, la zoologie, l'histoire et la philologie, par Pí́trenent. 1 vol. in-8. $8 \mathrm{fr}$.

Cheval. - Manuel hippique sommaire de l'éleveur cultivateur, enseignement professionnel dédié aux élèves adultes des Écoles rurales, par

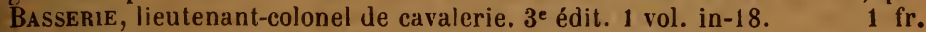
Approuvé par la Commission des bibliothèques scolaires.

Cheval. - Recherches sur la nature des affections typhoides du cheval. par Salle. 1 vol. in-18, orné de 30 fig. dans le texte. 350 Ouvrage couronné par la Société centrale de Médecine vétérinaire.

Cheval en France $(L e)$, depuis l'époque gauloise jusqu'à nos jours. Géographie et institutions hippiques, par E. Houes, 1 vol. in-8. $3 \mathrm{fr}$. Cheval de service. Production, élevage et dressage, par Ephrem Hovel, 1 vol. in-18. $1 \mathrm{fr}$. Chevaux. - Conseils aux éleveurs de chevaux, par Charles du Hays.

1 vol. in-18, fig.

350

Chevaux de chasse. - Leur condition en France, par le comte LE CouteulX de Cantelet. $2^{\circ}$ édit. 1 vol. in-18. $1 \mathrm{fr}$. Chien de chasse $(D u)$. - Chiens courants, espèces et variétés, élevage, dressage, maladies, extrait du Nouveau traité des chasses à courre et à tir. 1 vol. in-18 orné de 17 Gg. et d'un plan de chenil. $\quad 350$ Le même, imprimé sur papier vergé. Consells aux Chasseurs sur le tir, les armes, la Chasse en plaine et les différentes chasses d'oiseaux aquatiques, par H. Robinson. 2 édit. 1 vol. in-8 orné de pl. et de grav. dans le texte. $5 \mathrm{fr}$.

Donmages aux champs causés par le gibier, lapins, lièvres, sangliers, etc. - De la Responsabilité des propriétaires de bois et forêts,

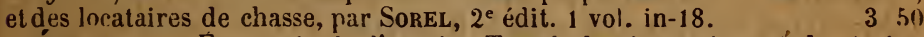
Écurie. - Economie de l'écurie. Traité de l'entretien et du traitement des chevaux, par J. Stewart, trad. de l'anglais sur la $7^{\circ}$ éd., par le baron D'Hanens. 1 vol. in-18 orné de $20 \mathrm{fg}$.

Fauconnerie ancienne et moderne, par CHENU et 0 . DES MURS 1 vol. in-18, orné de fig. 350

Maladies du cheval. Description et traitement, suivis de Notions de chirurgie vétérinaire et d'une pharmacie économique, par A. G*** 1 vol. in-18 orné de 23 fig. dans le texte.

$2 \mathrm{fr}$.

Pêche à la llgne. Conseils, par C. Jobey. 1 v. in-18, orné de 35 fig. 150

Pied de cheval $(L e)$ et la nanière de le conserver sain, par W. MiLes, trad de l'angl. par GuYron. 1 v. in $-8^{\circ}$ orné de pl. et de fig. dans le texte. 5 fr.

\section{Chasse, Chevaux, Chiens, Oiseaux de Volière, etc.}

Acheteur de chevaux. Guide pratique, par A. Rivet, ancien officier acheteur. $3^{e}$ éd., 1 vol. in-18. 250

Cailles, Perdrix, Colins ou cailles d' mérique. - Guide pratíque pour les élever, etc., par Allarx. Nouvelle édition 1 vol. in-18. Fig. (Nouvelle édition, en préparation).

Cavaller. Nouvelle école du cavalier ou l'art d'apprendre à monter à cheval sans sortir de son cabinet, par J. Mussieux. In-18. $1 \mathrm{fr}$.

Chasse au Gabion, par DIGUeT. In-18, fig. $1 \mathrm{fr}$.

Chasse en plaine, au bois, au marais. - Noureau guide pratique du petit chasseur, par E. NoDot. 1 vol. in-18 orné de pl. $4 \mathrm{fr}$. Cheval. Manuel à l'usage de nos amateurs de chevaux et des genre d'écurie, par un homme de cheval. $2^{\text {e }}$ éd., 1 vol. in-18 de 206 pages. 5 fr. Cheval. - La connaissance générale du cheval, par MM. Moll et Gayot, 3e édit. 1 vol. in-8 et un atlas de 103 fig.

$15 \mathrm{fr}$. Cheval $(E e)$ du laboureur et du soldat, ou le cheval de service en France, par Bouniceau. 1 vol. in-8 de 66 pages. $2 \mathrm{fr}$. 
Chevaux francais (Les) en Angleterre (1865), par E. Hovel. In-8. $2 \mathrm{fr}$.

Chien. - Traité pratique. - Hisloire; description des races de chasse françaises et étrangères, de garde et de défense, de troupeaux, de sauvetage, áagrément; reproduction, élevage, nourriture, logement: dressage ; hygiène; maladies internes et externes et leur traitement, par A. GoBiN. 1 vol. in-18 orné de 10 gravures.

350

Chien. - Le Chien, histnire naturelle, races d'utilité et d'agrément, reproduction, éducation, hygiène, législation, par GAY0T. 1 vol. in- $8^{\circ}$ avec atlas de 127 figures.

$12 \mathrm{fr}$.

Chiens. - Les Maladies des chiens et leur traitement, par Hertwig, 2 édit. 1 vol. in- 18 .

Courses. - Les Courses en France, en Belgique, etc., par CH. Du HaYs. 1 vol. in $\cdot 8^{\circ}$.

$5 \mathrm{fr}$.

Encastelure du pied du cheval, par Defays, professeur d'arvétérinaire. 1 vol. in-18 orné de $5 \mathrm{fgg}$. dans le texte. $75 \mathrm{c}$.

Faisans, Canards mandarins, Cygues, etc. - Guide pratique pour les élever, par Alfred Touchard. $2^{e}$ édit. 1 vol. in-18 avec fig. $2 \mathrm{fr}$.

Faisans, tragopass, crossoptilons, lophophores, etc. Manuel d'élevage, suivi d'une Monographie des Phasianidés, par DHerse. 1 vol. in-18.

$2 \mathrm{fr}$.

Fauconnerie. Précis contenant les indications nécessaires pour affaiter et gouverner les principaux oiseaux de vol, suivi de l'éducation du cormoran, par Sourbets et de Saint-Marc. 1 vol. in- $8^{\circ}$.

$5 \mathrm{fr}$.

Diseaux de volière (Manuel de l'Amateur des), ou Instruction pour connaître, élever, conserver et guérir toutes les espèces d'oiseaux que l'on aime à garder en volière ou dans la chambre, par BEchstein. (INouvelle éd., classée et revue par l'éditeur. $1 \mathrm{v}$. in-18 orné de $85 \mathrm{fig}$. dans le texte. 350

Poulains. - Manuel du petit éleveur de poulains dans le Perche, soins à donner aux poulinières, etc., par J. B. Huzard, 1 vol. in-18 orné de 4 fig. et de 2 planches lithographiées.

2 fr.

mevue cynégétlquo et sportive, paraissant le samedi de chaque semaine par livraisons de 16 pages in- $4^{\circ}$ illustrées. - Prix de l'abonnement pour l'année: France et Algérie. $15 \mathrm{fr} . ;-$ Europe. 1650 ; - Autres contrées, $19 \mathrm{fr}$. - Les abonnements partent du $1^{\text {er }}$ de chaque mois.

Vénerie. - Traité de vénerie, par D'Yauville. 1859. 1 vol. grand in-8 ${ }^{\circ}$ papier vélin, orné de 4 grandes gravures hors texte, de 9 fig. médaillons, et accompagné de 42 fanfares.

$25 \mathrm{fr}$.

Le même, papier ordinaire, sans gravures et sans fanfares.

$6 \mathrm{fr}$.

\section{Divers.}

Cent trente recettes pour apprêter le Iapin, ouvrage dédié aux personnes économes et gourmandes, par HACHEBÉE. 1 vol. in-18. $1 \mathrm{fr}$.

Cuisinlère $(L a)$ de la ville et de la campagne, par L. E. A. $45^{e}$ éd. 1 vol. in-18 cart. orné de 300 fig.

$3 \mathrm{fr}$.

Enquêtes administratives. - Traité et Formulaire, à l'usage de MM. les juges de paix, suppléants de juges de paix et maires, par E. NoEuvéGLISE, licencié en droit et juge de paix. In-8 .

$1 \mathrm{fr}$.

Etat civil. - Conditions et formalités pour la célébration de mariages. Formule à l'usage de MM. les maires et adjoints, par Toussaint. In- $4^{\circ}$. Cart. avec fers dorés sur le plat.

250

Gravure. - Des mordans, des vernis et des planches dans l'art du graveur, par DELESCHAMPS. 1836.1 vol. in-8ªccompagné de 3 planches gravées.

350

Mrénage. - Economie du ménage, ou principes d'économie populaire, par GERARDI. 1 vol. in-18. 150

pôtissier de la ville et de la campagne, par P. Quentin. $3^{\circ}$ édit. 1 vol. in-18 orné de $100 \mathrm{fg}$. dans le texte.

$$
\text { Paris. - Imp. L. Caplomont et } \mathrm{C}^{i \theta} \text {, rue des l'oitevins, } h_{,}
$$




$$
2^{\text {me }} \text { SÉRIE, No } 12 .
$$

\title{
BIBLIOTHÈUE RURALE
}

\author{
INSTITUÉE
}

PAR LE GOUVERNEMENT.

\author{
T R A I T E \\ DES \\ GRA IIINÉES.
}




\section{Typ. de J. Vanbuggenhoudt.}




\section{TRAITÉ}

DES

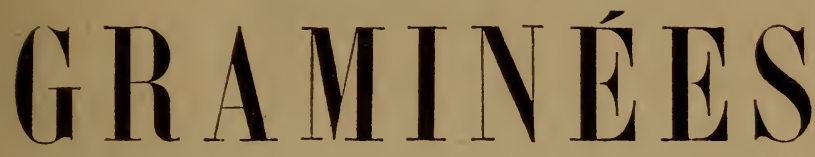

CÉréaleS RT FourRagères

QUE L'ON REXCONTRE EN BELGIQUE,

Avec des observations sur quelques variétés nouvelles,

PAR

V. P. G. DE MOOR,

MÉdECIN VÉTÉRINAIRE DU GOUVERNEMENT, SECRÉTAIRE DU CONICE AGRICOLE DU Se DISTRICT DE LA FLANDRE ORIENTALE, ETC.

Il faut donc apprendre à distinguer les bonnes des mauvaises espèces, à reconnaitre aussi celles qui sont inutiles ou indifferrentes, à choisir celles qui conviennent le mieux à telle ou telle espèce de bétail, étudier leur position dans les lieux où elles croissent naturellement, examiner celles qui leur plaisent le plus, approprier les espèces à la nature de son sol, et l'on verra alors qu'il y a réllement beaucoup de science dans une botte de foin.

(LECOQ; Traité des Plantes fourragères.)

\section{BRUXELLES,}

A LA LIBRAIRIE AGRICOLE DE H. TARLIER,

éditeur de la Bibliothèque rurale,

RUE DE LA MONTAGNE, $\mathbf{N}^{n} 51$.

1854 



\section{PREAMMBULE.}

La Bibliothèque rurale, qui s'adresse spécialement aux agriculteurs et aux propriétaires ruraux, peut-elle placer sous son patronage et comprendre dans ses publications des travanx que l'on pourrait considérer comme étant plutôt du domaine de la science botanique que de celui de la science ou de l'art agricole? Telle est la question qui nous a préoccupé un instant. Mais nous n'avons pas tardé à acquérir la conviction que, dans l'enseignement, un ouvrage de botanique agricole est au moins aussi indispensable qu'un manuel sur la culture des prairies, si, bien entendu, on veut allier la science à l'art, si l'on désire franchement que le progrès s'affranchisse de la routine et ne souffre plus, par conséquent, de la lenteur qui marqua naguère la connaissance et l'introduction de tous les végétaux utiles.

Il surgit ensuite une autre question : c'est celle qui est relative à la forme que doit revêtir cette publication. Quelques personnes bien intentionnées, mais étrangères à l'agroslographie, ont cru qu'on pouvait avec avantage lui donner un caractère purement pratique et se borner à quelques descripiions générales des espèces les plus utiles; mais il sera facile de concevoir qu'un pareil plan, s'il était mème exécutable, ne répondrait aucunement à l'attente des agronomes et des cultivateurs instruits qui roudraient acquérir la connaissance de toutes celles qui croissent sur le sol belge: D'ailleurs, ces 
descriptions générales et agricoles serviraient infailliblement à multiplier et à propager les causes d'erreur, sans le secours de très-bonnes figures coloriées, et mieux vaut mille fois ignorer la nature des objets que d'en avoir des idées fausses et erronées. Ce plan pourrait être adopté et suivi pour d'autres plantes où les analogies entre genres et espèces ne sont pas aussi étroites; mais pour les graminées céréales et fourragères, nous le répétons, il est absolument inadmissible. Aussi n'est-ce qu'après un mûr examen que nous avons entrepris la tâche que le Gouvernement nous a confiée, et que nous nous sommes arrèté à un plan qui, quoique plus ou moins scientifique, réunit à la clarté et à la simplicité la concision et la certitude.

Notre manuel est divisé en trois parties.

La première partie est consacrée à l'étude de la nomenclature des organes des graminées, des formes et des positions gu'ils affectent. Lorsqu'on se sera bien pénétré de cette nomenclature, le langage scientifique auquel nous avons cru devoir accorder la préférence pour éviter des périphrases interminables, et qui pourrait faire naitre quelque appréhension aux commençants, sera réduit à sa plus simple expression. Dès lors la deuxième partie n'a plus la moindre difficulté

La deuxième partie, outre la description complète des genres et des espèces (1), comprend quelques tableaux dichotomiques (2) qui facilitent les recherches des tribus, des genres et des espèces.

En effet, si l'on veut découvrir les noms d'une

(1) Les espèces dont la description est suivic d'un point d'interrogation n'ont pas élé rencontrécs en Belgique depuis au moins dix ans.

(2) La métliode dichotomique consiste dans le mécanisme suivant: Un certain nombre d'objets étant donnés, il s'agit de conduire successivement an nom de chacun d'eux; pour y parvenir, on les divise en 
plante quelconque, on n’a qu’à déterminer d'abord
à quelle tribu elle appartient; à passer ensuite à

deux groupes limités par des caractères bien tranchés, puis on subilvise chacun des deux groupes en deux groupes secondaires et ainsi le suite jusqu'à ce que fion arrive à l'unité, soit collective, soit individuelle dont on cherche le nom (Germans).

Nous ferons comprendre la clef des tableaux dichotomique par un exemple : nous supposerons qu'on veuille dénommer le scigle cultivé.

On détermine d'abord à quelle tribu il appartient; à cet effet on ouvre la deuxième partie, analyse des tribus. On lit les deux paragraphes compris sous laccolade 1 .

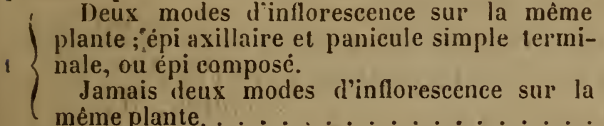

La plante ne présentant pas deux modes d'inflorescence, on passe à laccolade 2 à laquelle renvoie le chillre.
( Axe primaire de l'inflorescence simple très- rarement rameux présentant sur une face ou
2
deux faces presqu'opposées des dépressions ou
des excavations correspondant aux locustes ou
rachis en zigzag, ariiculè et denté . . . . . Tniticées. (II).
Plantes ne réunissant pas ces caractères.

L'axe primaire de l'intlorescence étant simple et présentant des dépressions correspondant aux locustes on a en main une plante appartenant à la tribu des Triticées II.

La tribu étant trouvée, on cherche à découvrir le genre au tableau de l'analyse des genres. $1\left\{\begin{array}{l}\text { Deux ou trois locustes sur chaque dent du } \\ \text { rachis. }\end{array}\right.$

Une locuste sur chaque dent du rachis . .

5.

Notre plante ne présentant qu'une locusle sur chaque dent, on passe à l'accolade 3 .

$3\{$ Glume nulle.

A cause de la glume l'on passe à l'accolade 4 .

4 Paillettes munies de trois à cinq arètes.

$+\{$ Paillettes mutiques ou munies d'une arête. .

4.

La paillette ne présentant pas trois à cinq áêtes l'on passe à l'accolade כ.

. $\left\{\begin{array}{l}\text { Glume à une paillette; locuste présentant le } \\ \text { dos au rachis. } \\ \text { Glume à deux paillettes; locuste présentant } \\ \text { l'une des faces latérales au rachis. . . . . }\end{array}\right.$

6.

La glume étant à deux paillelles et les locustes présentant l'une des faces latérales au rachis l'on passe à l'accolade 6 .

6 Locuste à un fleuron fertile.

6 Locuste biflore on multiflore........ 7.

La locuste présentant deux fleurons et un rudiment de troisième fleuron, l'on passe à l'accolade 7 . 
l'analyse des genres de la tribu et à chercher enfin dans le tableau qui suit, l'énoncé complet des caractères de chaque genre et le nom de l'espèce.

Pour vérifier l'exactitude de la détermination, on lit la description du genre auquel renvoie un numéro d'ordre, et ensuite celle del'espèce; la lettre qui suit le nom de l'espèce, et qui est mise entre parenthèses, correspond à celle de la description détaillée, suivie de quelques indications sommaires des lieux où on la rencontre.

Dans la troisième partie, enfin, nous indiquons tout ce qu'on sait sur les stations, les propriétés et le rendement des espèces et des variétés, d'après les observations consignées dans les meilleurs travaux récents sur l'économie rurale.

Une table alphabétique, où chaque partie a sa pagination spéciale avec la synonymie complète des espèces utiles, pour faciliter les recherches, termine l'ouvrage.

Nous espérons que ce manuel contribuera dans un cercle étendu à propager la connaissance des plantes qui entrent pour une si large part dans la richesse nationale, et nous nous estimerons toujours heureux de pouvoir coopérer à aplanir la voie qui conduit directement ou indirectement à des améliorations agricoles dont les résultats rejaillissent surle bien-être matériel et moral de la nation.

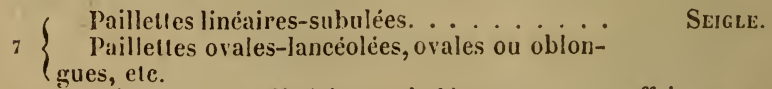

Les pailleltes étant linćaires-subulées nous avons affaire au genre scigle qui est le quatrième dans la partie descriptive. Ce genre ne comprenant qu'une seule espèce, celle-ci est découverte et nommée scigle cultive.

S'il y avait plusieurs especes de ee genre on en trouverait l'analyse dichotomique après la description complète de celui-ci, el pour en savoir le nom on s'y prendrail comme pour l'analy'se des tribus et des gremies. 


\section{PREMIÈRE PARTIE.}

INTRODUCTION

$A$

\section{L'ÉTUDE DES GRAMINÉES.}

NOMENCLATURE.

L'histoire des graminées se rattache étroitement a l'histoire de la civilisation; partout où celle-ci a fait quelques progrès, l'on trouve leur culture en honneur. Elles forment, aux yeux du botaniste, la famille la plus riche et la plus naturelle de tout le règne végétal, et l'agronome, le cultivateur, lui, la considère, à juste titre, comme la plus importante de ses cultures et comme le baromètre de l'état plus ou moins prospère où se trouve un pays.

En effet, toutes peuvent servir à nos besoins ou concourir à nos jouissances : ici elles forment la base de la nourriture de l'homme; là, elles pourvoient à la subsistance et à l'entretien de ses troupeaux, et fournissent les éléments de diverses industries, enfin; plus loin, leurs tiges souterraines raffermissent et fixent les sols mobiles de pays entiers.

Les graminées appartiennent à toutes les stations; on les trouve sur les hauteurs comme dans les plaines, sur les penchants des collines comme dans les bas-fonds, dans l'eau comme dans les 
plaines arides et sablonneuses, et elles n'abordent IIn lieu que pour le féconder; mais toutes les espè. ces de sols, toutes les stations, quelque variées qu'elles soient, ont, en quelque sorte, leurs espèces propres, sinon comme types botaniques, du moins comme types agricoles.

\section{I. - de la tige et des racines.}

Toutes les graminées qui sont cultivées en Belgique, ou qui y croissent spontanément, sont herbacées (de la nature de l'herbe, c'est-à-dire ce qui n'acquiert pas la consistante du bois et qui périt après quelques mois de végétation.

Annuelles, (qui se développent, fructifient et périssent au bout d'une année.)

Bisannuelles (qui périssent à la deuxième année de végétation).

Vivaces, (qui vivent au moins pendant trois ans.) Les tiges se développent et meurent tous les ans. Les racines et la partie qui doit émettre les chaumes, qu'on appelle souchie, vivent un certain nombre d'aunées.

Leurs racines sont fibreuses et plus ou moins rameuses (on appelle racines fibreuses, celles qui sont longues, assez grèles et distinctes); elles peuvent ètre simples ou ramouses : elles sont simples lorsqu'elles ne donnent lieu à aucune division secondaire; elles sont rameuses lorsqu'clles forment des divisions secondaires, tertiaires, ete., de moins en moins épaisses.

Les tiges des graminces sont soutervaines ou aériennes.

Les tiges souterraines sont celles qui se développent dans la terre; ces tiges ont été confonduer 
longtemps avec les racines rampantes. Chez les graminées il n'y a pas de racines rampantes; les tiges souterraines comme les tiges aériennes présentent des espèces de nœuds : c'est de ces nœuds que l'on voit naitre des racines. L'on y remarque aussi des écailles, rudiments de feuilles; à l'aisselle de chaque écaille l'on découvre un bourgeon, et c'est ce bourgeon qui est la cause de la facilité avec laquelle se reproduit la moindre portion de tige souterraine appartenant à un assez grand nombre d'espèces que les agriculteurs désignent sous le nom collectif de chiendent. La tige souterraine a été appelée rhizome par Bellenden Ker : cette expression, si l'on s'en refère à l'étymologie du mot, est des plus impropres : les rhizomes sont souvent pleins ou presque pleins.

La figure suivante A représente une portion de lige souterraine du chiendent dactyle. 
MANUEL DES GRAMINEES.
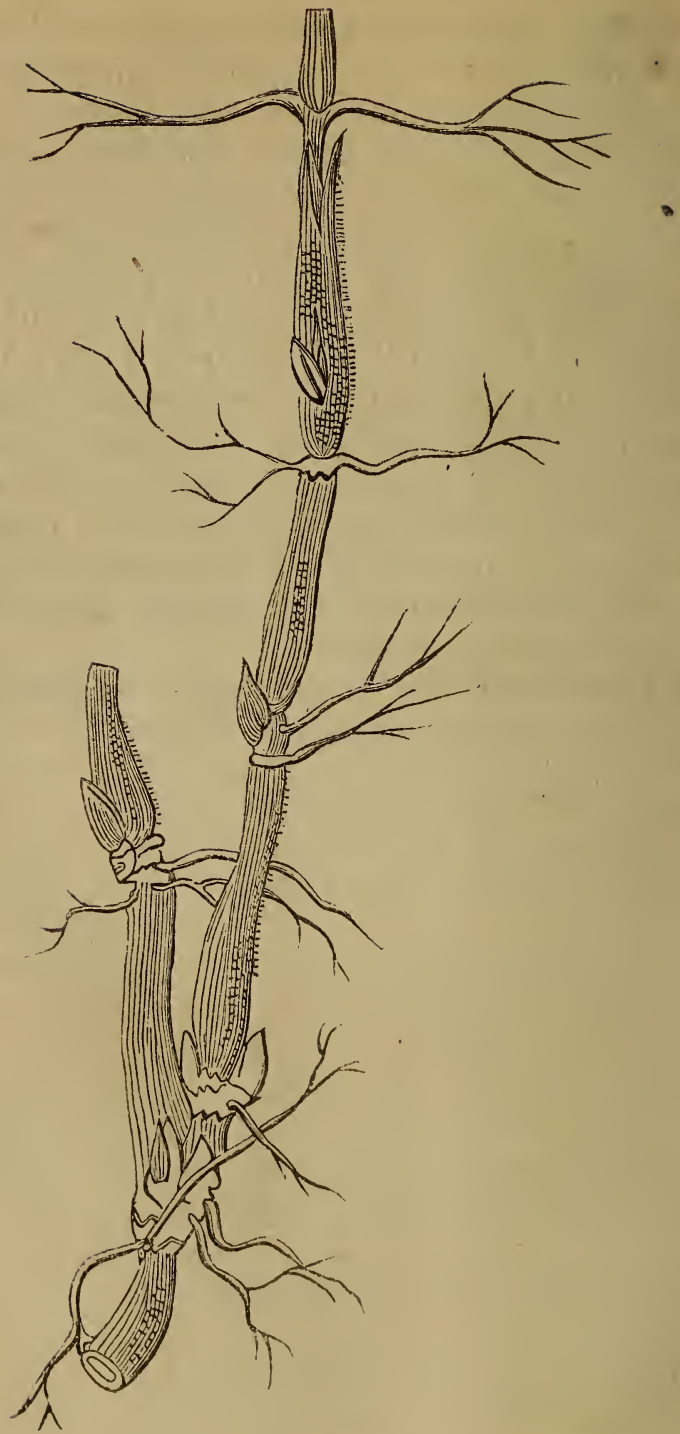

ing. 1. 
Les tiges aériennes sont celles qui se développent à l'air. Ainsi, toutes les tiges qui naissent de la souche mère de la génération actuelle, et qui se inontrent au-dessus du sol, appartiennent aux tiges aériennes que l'on appelle chaume.

Le chaume esi l'axe qui supporte les bourgeons, les feuilles et les fleurs.

La base du chaume est quelquefois renflée en forme de bulbe (fig. B), simple ou multiple. Ces bulbes qui opèrent alors la transition de la tige aérienne à la tige souterraine, affectent diverses formes : tantôt ils sont presque en forme de sphère ou de boule (fig. B \& a); d'autres fois ils sont un peu allongés et amincis vers le haut, de manière à leur imprimer l'aspect d'une poire (fig. B $1 b$ ), et d'autres fois, enfin, ils sont aplatis et ressemblent à des oignons plats, enfilés à peu près comme les grains d'un chapelet (fig. B 2). Ces bulbes sont couverts en partie ou en totalité par une feuille rudimentaire, comme on le voit sur la figure suivante; de l'avoine élerée. (Arrhenaterum avenaceum P. D. B. rariété bulbeuse).

Les chaumes sont simples ou rameux, droits ou obliques, couchés ou décombants, ordinairement fistuleux ou creux supérieurement et même dans la plus grande partie de leur longueur ; ils sont assez rarement pleins ou remplis de moelle analogueà celle que présentent la plupart des joncs. Le froment turgide auquel se rapportent la pétanielle noire,etc., et le froment dur, etc., ont le chaume plein.

Ils sont coupés de distance en distance par des fragments solides qui forment à l'intérieur un plancher très-dense et résistant. Ce plancher est biconcave, c'est-à-dire qu'il présente sur ses deux faces une dépression d'autant plus prononcée qu'on 
MANUEL DES GRAMINÉES.

approche plus du centre; ce plancher correspond extérieurement à un renflement qui est connu sous

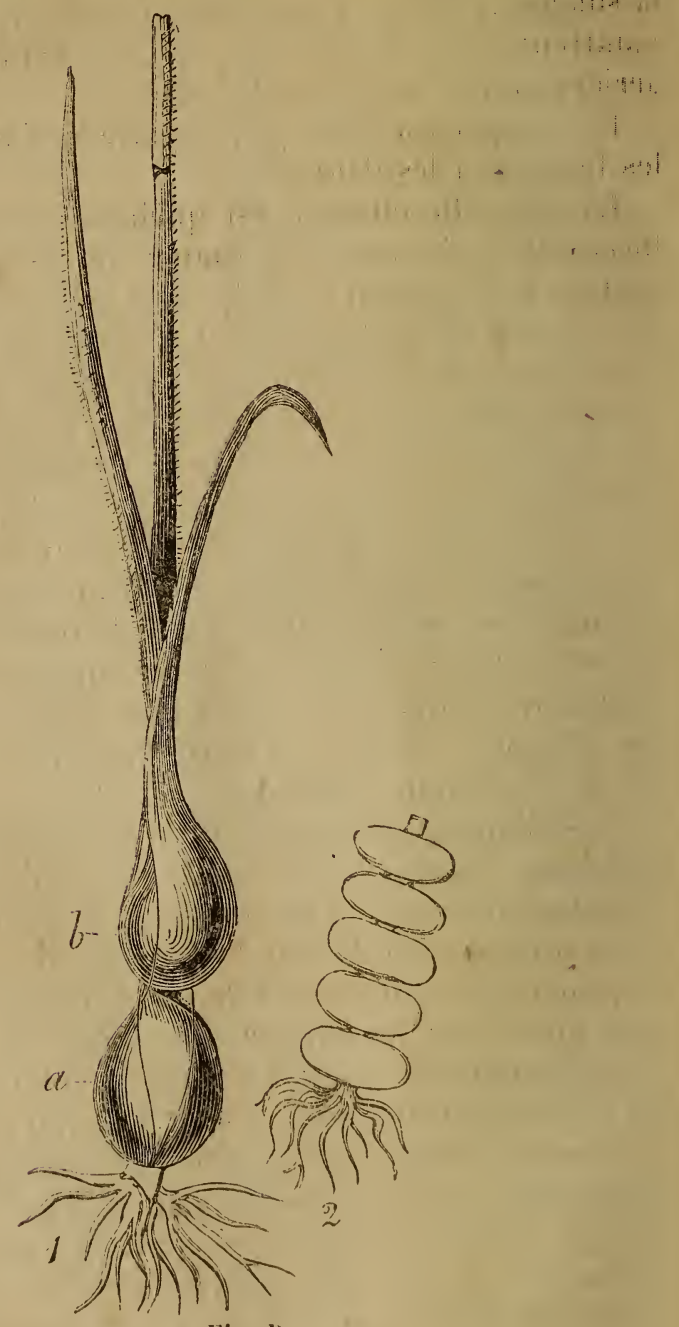

Fig. 13. 
le nom de nœud. Le nombre des nœuds varie beaucoup; ils sont plus rapprochés dans le bas que dans le haut de la tige. Les nouds font rarement défaut dans la portion aérienne. On ne connait qu'une seule grande espèce indigène, (l'énodie bleuâtre,) où les feuilles sont insérées à la base des chaumes provenant de la souche : cette base présente des nouds si rapprochés qu'il est assez difficile de découvrir l'origine d'insertion de chaque feuille.

Chaque noud présente un bourgeon plus ou moins développé, qui sert à la multiplication de l'individu, quand on le place dans des conditions convenables; ces bourgeons se trouvent à l'aisselle des feuilles et affecient la même disposition extérieure que celles-ci.

La portion comprise entre deux noêuds s'appelle entre-noud ou mérithalle.

Le chaume, comme la tige de presque tous les végétaux, s'amincit graduellement de la base au sommet. Dans quelques espèces, cet amincissement ne commence qu'à quelques centimètres au-dessus de l'origine du chaume.

Le chaume peut être :

Cylindrique, lorsque sa circonférence est représenté par un cercle;

Comprimé, quand il est aplati suivant deux points qui sont opposés entre eux;

Ancipité, quand il est aplati et que ses deux côtés sont aigus et presque tranchants ;

Tétragone, lorsqüil présente quatre angles et autant de faces plus ou moins clairement dessinés.

\section{II. - DES FEUILLES.}

Les feuilles, qui ne semblent être que des expansions alongées naissant du pourtour des 
nouds, et dont elles ne paraissent être que des pro. longements, sont distiques, eu égard à la disposition du bourgeon dont nous avons parlé plus haut.

Ce terme indique que les feuilles sont rangées en deux séries disposées le long de la tige et dans le même plan, mais à des hauteurs différentes, de manière qu'il y en ait alternativement une d'un côté et l'autre de l'autre côté. Ces feuilles sont donc alternes et disposées sur deux rangs. En effet, en jetant un coup d'œil sur un chaume quelconque muni de ses feuilles, on se convaincra que la troisième feuille est insérée immédiatement audessus de la première.

Les feuilles sont dites radicales (1) ou caulinaires.

Les feuilles radicales sont celles qui naissent non loin du collet des racines ou très-près du sol, et qui sont déjà très-distinctes avant que le chaume ait acquis quelque développement : tout bourgeon souterrain produit donc nécessairement des feuilles radicales. Les caulinaires naissent distinctement et à une certaine hauteur sur le chaume.

Les feuilles présentent trois parties, savoir :

a. La gaîne (fig. C 1).

b. La ligule (fig. C 2).

c. Le limbe ou la lame (fig. C 5 ).

La gaine est une espèce de pétiole qui embrasse le chaume, en forme d'étui, à nervures parallèles. Cette partie est nulle ou presque nulle dans les feuilies dites radicales; elle est ordinairement trèsdéveloppée dans les feuilles caulinaires. La gaine est tantôt fendue dans toute sa longueur; d'autres fois, elle est fendue jusqu'au-dessous de la partie

(1) M. Seringe les apprlle avec plus de raison fouilles infericures. 
moyenne; enfin, ellc est entière ou seulement fenciue all sommet.

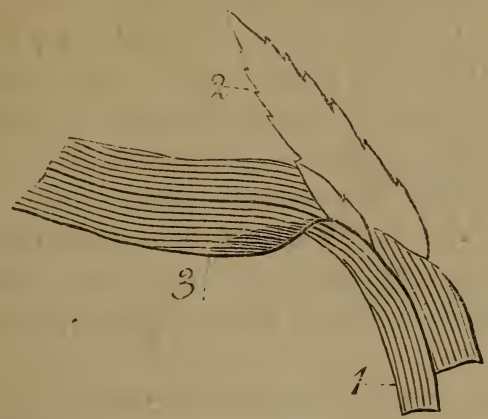

Fig. C.

La gaine est fendue jusqu'à la baše, lorsqu'on pent la dégager du chaume sans produire aucune ¿léchirure. Ce dégagement s'opère en la déroulant, attendu qu'elle est roulée en cylindre autour du chaume; dans ce cas, ses bords sont libres et sa largeur dépasse celle de la circonférence du chaume. (Toutes les céréales.)

Lorsqu'elle n'est fendue que jusqu'au-dessous de la partie moyenne, sa largeur dépasse à peine la circonférence du chaume, et les bords de la partie fendue ne se recouvent que peu (Glycérie flottanie). Lorsque la gaine est entière, sa largeur excède très-peu la circonférence des chaumes, et les bords sont sourent soudés entre eux jusque près de la naissance du limbe. (Mélique uniflore, $\mathbf{R}$.)

La gaine est tantôt plus longue que le mérithalle (énodie), d'autres fois plus courte.

La gaine neut ètre arrondic, comprimée, ancipitée. 
La ligule, qu'Adanson a nommée languette, esı le plus souvent une membrane mince, située sur la ligne qui sépare la gaine du limbe (ligule membraneuse); d'autres fois, la ligule, au lieu d'être membraneuse, est remplacée par une ligne de poils disposés sur un ou plusieurs rangs : (ces poils sont constamment simples)(ligule pileuse); d'autres fois, enfin, la ligule fait complétement défaut, et l'on remarque à la place qu'elle devrait occuper, une espèce d'étranglement ou une ligne autrement colorée que le reste du tissu (ligule nulle ou rudimentaire).

La ligule membraneuse affecte diverses formes ou figures. On la dit :

Oblongue, lorsqu'elle est beaucoup plus longue que large;

Oblongue-lancéolée, quand elle est plus longue que large et que l'extrémité se rétrécit insensibleinent (Paturin commun).

Courte, lorsque sa largeur dépasse sa longueur.

Eu égard à son sommet, la ligule peut être :

Aiguë, quand elle se termine par une pointe courte se confondant insensiblement avec le corps de la ligule.

Acuminée, lorsqu'elle est terminée insensiblement par une pointe plus ou moins longue.

Mucronée, lorsquele sommet est obtus et terminé brusquement par une pointe plus ou moins allongée.

Bifide-trifide, quand elle est divisée en deux ou trois parties; chaque partie peut ètre acuminée, mucronée, aiguë, etc.

Laciniée, lorsqu'elle est divisée en plusieurs lanières plus ou moins larges et nombreuses.

Dentée, lorsqu'elle présente quelques petites dents. 
Obtuse, lorsque le sommet est arrondi.

Tronquée, quand elle se termine brusquement par une ligne horizontale et qu'il semble par conséquent qu'on en a coupé le bout.

\section{Eu égard à sa vestiture, elle peut être :}

Nue, lorsqu'elle ne présente ni duvet, ni poils.

Ciliée, quand elle présente des poils sur son contour.

La ligule pileuse peut ètre:

Duvetée, lorsque les poils sont très-courts quasi entremêlés et très-mous.
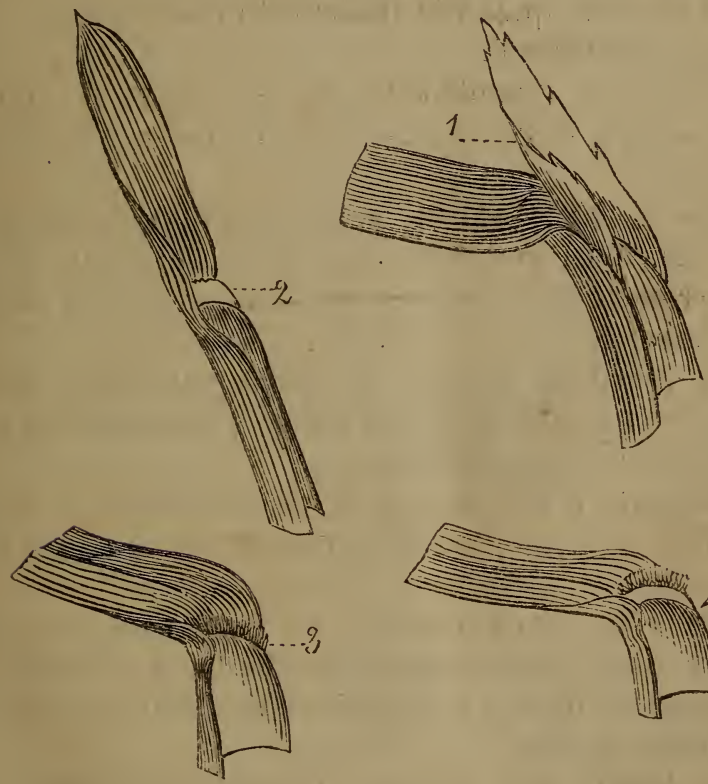

Fig. D. (1).

(1) Ligule membraneuse oblongue-lancéoléc; 2 ligule membraneuse courte; jigule pileuse; 4 ligule mixte 
Poilue, quand les poils sont assez longs, soyeux et distincts, quoique plus ou moins abondants.

La ligule est mixte, lorsque la base, très-courte, est de nature membraneuse et que son contour est garni de poils nombreux : cette espèce de ligule établit la transition entre la ligule membraneuseciliée et la ligule pileuse.

Le limbe est la partie libre de la feuille. Sa direction est opposée à celle de la fente de la gaine.

Le limbe peut être :

Lancéolé, linéaire, subulé. (Voir les définitions à l'article Paillettes).

Rubanaire, quand le limbe est linéaire mais extrêmement allongé.

Filiforme, quand le limbe est presque rond, cylindrique et que sa grosseur n'excède guère celie d'un fil.

Setacé, celui qui est grêle, roide, qui a quelque analogie avec une forte soie de cochon; ce limbe est arrondi ou anguleux et s'amincit de la base au sommet.

Eu égard au sommet, le limbe peut être obtus, aigu, acuminé, mucroné, etc. (Voir les définitions à l'article paillettes.)

Eu égard à leur force et à leur résistance, il cst :

Mou, flasque, lorsqu'il ne peut se soutenir de soi-mème.

Roide ou ferme quand il est allongé et qu'il se relève avec force et élasticité lorsqu'on le courbe.

Cassant, quand il se rompt d'une manière nette lorsqu'on le plie.

Le limbe des feuilles, quoique en géneral, linéaire et plan, fournit cependant de bons caractères à l'agronome pour distinguer entre elles plusieurs espèces d'un mème genre. Ainsi, si l’on 
observe' le développement du limbe, on trouve yu'il n'est pas toujours, dans sa jeunesse, disposé de la même manière chez toutes les espèces. Tantôt le limbe est plié en deux, d'autres fois il nait enroulé sur lui-mème. Tous les paturins présentent des feuilles pliées dans leur jeunesse, de mème que le dactyle glomeré, l'avoine pubescente, la triodie, la glycérie, le foin aquatique (calabrose aquatique), etc. L'ivraie vivace se distingue de toutes les autres espèces du mème genre parce qu'elle a des feuilles pliées (fig. E 2) dans sa jeunesse, tandis qu'elles sont enroulées (fig. E bis 1) chez les autres. Toutes les avoines cultivées pour leur grain présentent des feuilles enroulées.

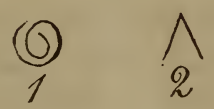

Fig. E.

Le contour de la lame peut ètre :

Entier, lorsqu'il ne présente ni dentsni aspérités.

Denticulé ou scabre, quand il offre de petites dents ou de petites aspérités.

Le chaume, la gaine et le limbe de la feuille peuvent ètre :

Striés, lorsqu'ils sont parcourus dans leur longueur par de petits sillons superficiels n'atteignant pas la moitié de l'épaisseur de l'organe, parallèles et longitudinaux.

Sillonnés, lorsqu'ils sont marqués de cannelures parallèles atteignant ou dépassant la moitié de l'épaisseur des organes. 
Unis, quand il n'y a aucune sorte d'aspérité ou d'èminences.

Glabres, lorsqu'ils sont dépourvus de toute espèce de poils.

Lisses, quand ils sont unis et glabres au toucher.

Glauques, lorsqu'ils semblent couverts d'une poussière vert de mer (les surfaces glauques peuvent se plonger dans l'eau sans qu'elles se mouillent).

Scabres ou rudes, quand la surface offre au doigt des aspérités insensibles à la vue simple; cet état est dû à des poils très-courts ou à de petites éminences; ces éminences affectent toujours une disposition oblique, ce qui fait que les surfaces sont rudes lorsqu'on y passe la main dans un sens donné, et qu'elles sont lisses lorsqu'on y passe le doigt dans un seus opposé.

Pubescents, lorsqu'ils sont garnis de poils mous, très-fins, assez courts et un peu clair-semés.

Poilus, quand ils sont couverts de poils mous et peu nombreux.

Velus, quand les poils sont mous, longs, un peu obliques et très-rapprochés.

Laineux', lorsqu'ils sont munis de poils longs, un peu crépus, semblables à de la laine : on les désigne encore sous le nom de poils aranéeux, à cause de l'analogie qu'ils présentent avec les fils d'une toile d'araignée.

Cotonneux, quand les poils sont blancs, longs, crépus et doux au toucher comme le coton : ils forment une sorte de feutre.

Soyeux, lorsque les poils sont doux au toucher, luisants et non entremêlés comme le sont les fils de soie.

Hispides, quand ils sont garnis de poils longs, roides el à base tuberculée. 
Subaculéiformes, lorsquillssont munis d'aspérités crochues plus ou moins développées.

\section{III. - DE LA DISPOSITION DES FleURS}

La disposilion des fleurs a lieu de diverses manières; on peut la réduire à deux modes principaux d'inflorescence. Tout d'abord elle est axillaire (1) lorsqu'elle se trouve à l'aisselle d'une feuille, ou terminale, lorsqu'elle termine le chaume. L'inflorescence axillaire est extrèmement rare. Un exemple nous est fourni par une plante bien connue, le maïs cultivé. La même plante fournit aussi un exemple d'inflorescence terminale.

L'inflorescence a lieu en épi ou en panicule. Si l'on prend un épi de froment ou de seigle et une panicule d'avoine cultivée, la ligne de démarcation entre ces deux inflorescences est nette. Mais il n'en est plus de mème lorsqu'on veut appliquer rigoureusement le mot épi ou panicule à telle ou telle inflorescence à laquelle ne s'applique plus de tous points la définition qu'on a donnée de ces inflorescences. La nature ne comporte pas cette rigueur et ne se plie pas au langage rigoureux de la science; car il est des espèces dont la moitić ou les deux tiers de l'inflorescence appartiennent franchement à l'épi et le reste à la panicule. La fétuque fausse ivraie, qui est assez commune aux bords

(1) Cette expression a été introduite à tort dans l'agrostographie , car beaucoup de graminées émettent des rameaux caulinaires qui sc terminent quelquefois par une panicule ou un épi: or, le spadix du maïs n'est que la terminaison d'un pareil rameau; l'éragrostide leptostachye présente des chaumes très-ramcux; presque tous les rameaux se terminent par une panicule qui reste souvent incluse dans la gaîne de la feuille à laiselle de laquelle le rameau florifère a pris naissance. Quoique ce ne soit pas ici le lieu d'examiner ce point de science, nous avons cru cependant revoir en dire un mot. 
des fossés dans les prairies grasses, en donne un exemple frappant.

Quoi qu'il en soil, il faut réduire ces inflorescences à deux modes qui auront pour point de départ l'existence d'un axe simple ou ramifié. Si l'axe est

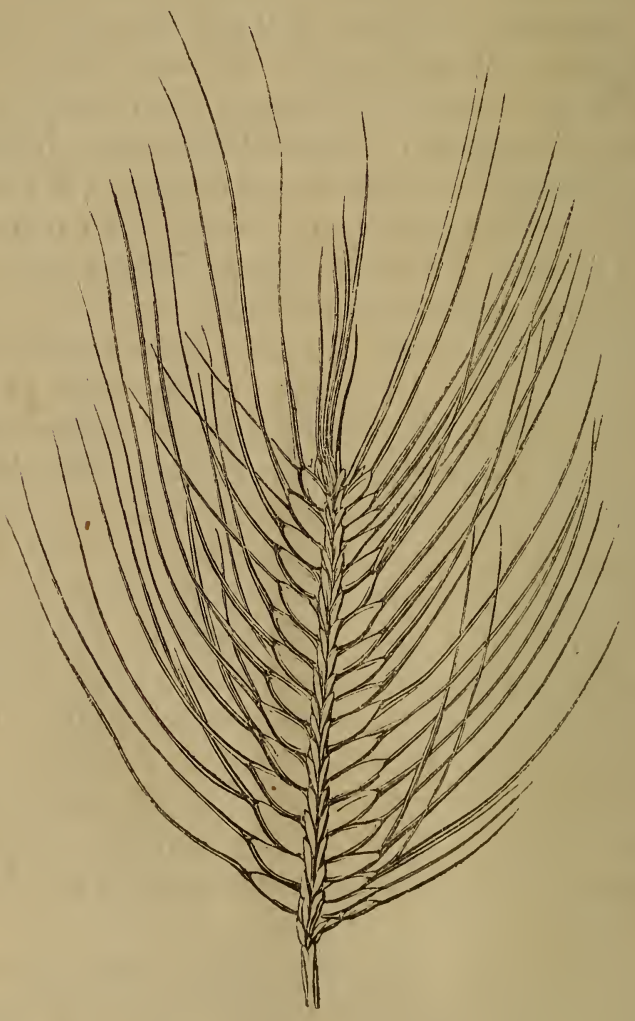

Fig. F.

simple et qu'il porte les divisions fleuries, composées d'un ou de plusieurs fleurons qu'on nomme 
locustes ou épillets, et que ces divisions s'insèrent sur lui à l'aide d'une très-courte queue ou pédoncule (qui est un rameau très-raccourci), l'inflorescence est un épi simple (fig. F). Si cet axe se divise en

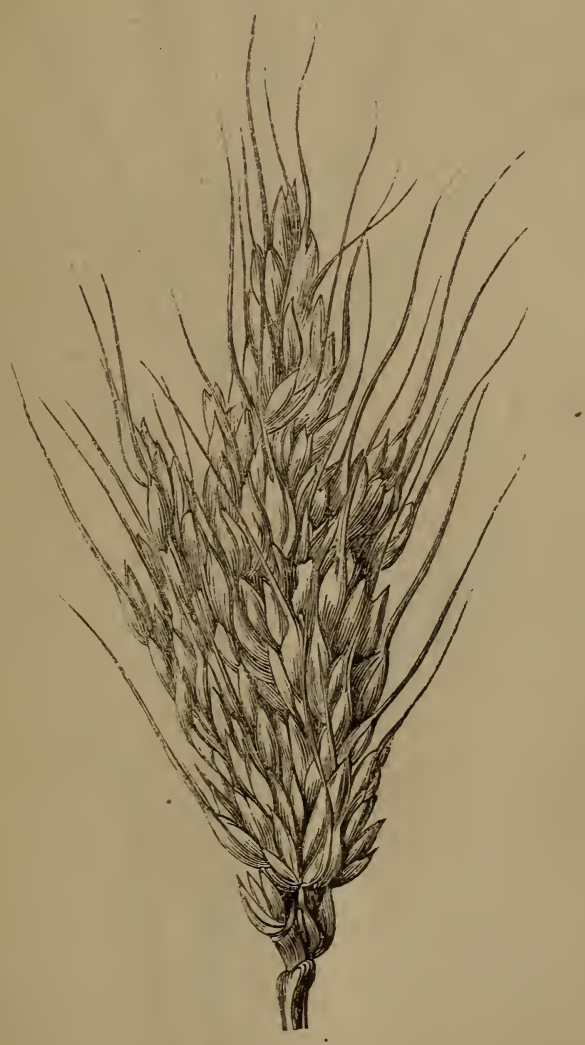

Fiư G.

plusieurs axes secondaires, et que ces axes secondaires portent des locustes très-courtement pédon- 
culées (la longueur des pédoncules ne dépassant pas celle de la locuste), ou s'insèrent sans le secour's

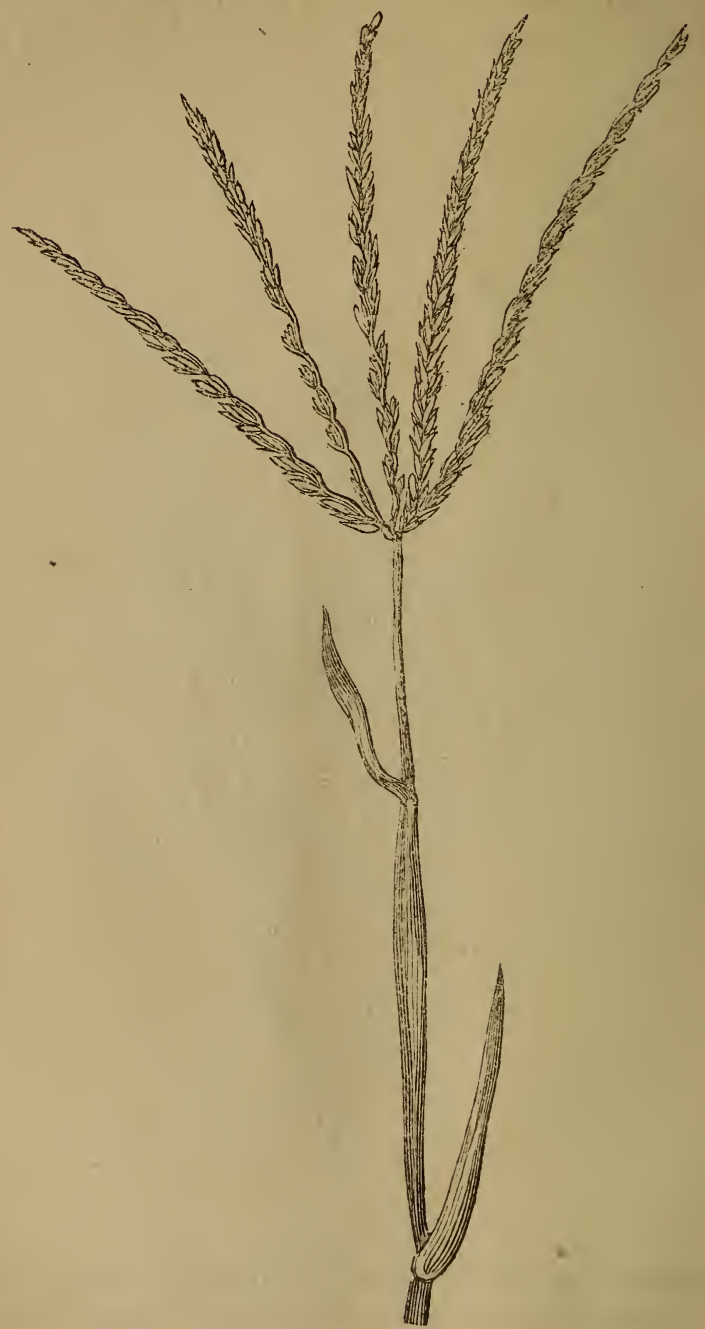

Fig. II. 


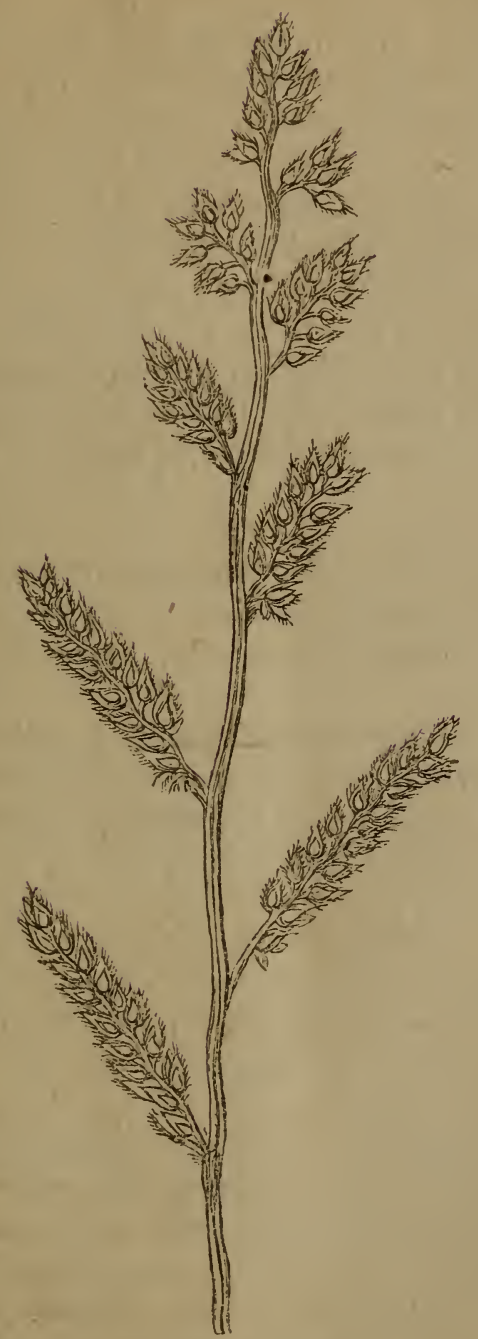

Fig. I. 
d'aucun pédoncule sur l'axe en question, la locuste est dite sessile. Lorsqu'il y a deux locustes, l'une d'entre elles doit au moins être sessile ou-subsessile, et l'inflorescence prend le nom d'épi composé. L'épi composé peut être compacte ou lâche : dans le premier cas, on se bornera à dire que l'épi est composé (fig. G). Lorsque l'épi est lâche, ses divisions peuvent simuler des épis distincts partant tous d'un même point, ou à peu près, et qui, ayant plus ou moins de roideur, sont diversement écartés les uns des autres, de manière à représenter des espèces de digitations; d'où le nom d'épi digité ou de panicule simple digité. (Fig. H.) (Chiendent dactyle.)

L'épi composé ailé (fig. I) est celui où les divisions naissent à des hauteurs différentes, mais presque disposées sur deux rangs (oplismène); chaque division secondaire porte le nom d'épiet.

L'axe sur lequel s'insèrent les locustes dans l'épi simple porte le nom de réceptacle, et le réceptacle dans le langage descriptif s'appelle rachis.

Le rachis est denté et articulé (voir fig. 3 B) ou en zigzag (fig. $5 \mathrm{~A}$ ); il présente une espèce de concavité correspondant à la locuste, et à la base de cette concavité une espèce de dent où s'insère chaque locuste. En faisant une section longitudinale au rachis, on remarque sur la coupe des points plus compactes, les uns que les autres. Les parties compactes répondent à l'insertion des locustes, ellesne sont pas autre chose que des nouds ou fausses articulations, comme on les qualifie assez généralement. Ce caractère ne se retrouve pas avec la mème netteté dans l'épi composé, digité ou ailé; aussi ne sommes nous pas éloigné de ranger 
ce genre d'inflorescence daus la catégorie des panicules.

Le rachis est fragile quand il se divise à la maturité en autant d'articles qu'il y a des dents d'insertion; et solide, persistant ou non fragile, lorsque cette division n'a pas lieu.

L'épi est régulier lorsque les locustes sont arrangées avec symétrie sur l'axe. (Froment, seigle, etc.)

L'épi régulier peut être :

Comprimé, quand il est fortement aplati;

Tétragone, s'il présente quatre faces et autant d'angles : ceux-ci sont ordinairement peu marqués;

Cylindrique, quand il est alongé et rond comme un cylindre.

L'épi est irrégulier lorsque cette symétrie est troubléc, et il esi unilatéral ou racémiforme lorsque les locustes sont toutes ou la plupart tournées du mème côté. (Nard élancé.)

L'inflorescence est dite en panicule lorsque l'axe primaire de l'inflorescence ou réceptacle se subdivise en pédoncules rameux.

Le réceptacle de la panicule est désigné sous le nom d'axe.

La panicule est rẻgulière ou irrégulière.

La panicule régulière est celle dans laquelle les divisions sont dirigées dans tous les sens; elle est irrégulière lorsque celles-ci n'affectent pas cette disposition :

La panicule régulière et la panicule irrégulière peuvent présenter des rameaux :

Étalés ou diffus, lorsqu'ils forment presqu'un angle droit avec l'axe;

Réfléchis, quand ils se dirigent en bas en formant arec la partie inférienre de l'axe un angle aigu. 
Divariqués, lorsqu'ils s'écartent les uns des autres dans tous les sens sans former des angles trèsouverts.

Effilés, quand ils forment un angle très-aigu avec la partie supérieure de l'axe.

La panicule est dite racémiforme ou unilatérale (fig. 87) lorsque les locustes sont dirigées la plupart vers un seul côté. Cette dénomination a aussi été donnée à la panicule simple, lorsque les locustes sont plus ou moins longuement -pédonculées. (Triodie decumbente. Melique penchée.)

Elle est spiciforme, (fig. 94, 101, 103, etc.), quand elle est contractée de manière à simuler un épi plus ou moins compacte (flouve odorante, kolérie crêtée, phalaride des Canaries, vulpin des prés). (Voir les fig. dans la $3^{\mathrm{e}}$ partic.)

La panicule peut être :

Ovale, oblongue, lancéolée. (Voir la définition de ces mots à l'art. paillettes.)

Pyramidale, quand elle se retrecit de la base au sommet.

Capitée, lorsqu'elle a la formed'une tète ou d'une petite boule.

Cylindrique, quand elle la forme d'un cylindre et partout à peu près le même diamètre.

\section{IV. - DES DIVISIONS FLeURIES.}

La locuste, ainsi qu'il a été dit plus haut, est la division terminale fleurie de la panicule ou de l'épi. La locuste peut se composer d'un ou de plusieurs fleurons : quand elle ne se compose que d'une fleur, elle est dite uniflore; quand elle en renferme de 2 à 4 , on la dit pauciflore; quand elle en renferme de .5 à 50 ou plus, elle est multiflore. 
organes pris collectivement prennent le nom d'involucre.

Il arrive que les fleurons de certaines locustes avortent et se présentent sous la forme d'une bractée ou organe subfoliacé. Deux genres indigènes présentent cette particularité : dans l'un, on trouve a la base de certaines locustes fertiles ou sur leur pédoncule une bractée pectinée (une bractée pectinée est celle qui est composée d'un certain nombre de lanières parallèles entre elles, ce qui lui donne l'aspect d'un peigne)(cynosure crételle); dans l'autre, c'est une simple bractée plus ou moins allongée (seslerie).

Les locustes sont toutes assez longuement pédonculées ou bien réunies sous forme de glomerules à cause du raccourcissement des pédoncules. Elles peuvent ètre ovales, elliptiques, lancéolées, linéaires. (Voir les définitions à l'art.Paillettes). Les locustes se composent d'une enveloppe commune à tous les fleurons de la locuste complète et au moins d'une enveloppe particulière qui entoure les organes reproducteurs.

$$
\text { V. - DE LA GLUne. }
$$

L'enveloppe commune et externe de chaque locuste est désignée sous le nom de glume. (Voir fig. J. Locuste uniflore, locuste pauciflore et multiflore, 1,) calice, bâle, lépicène, glumelle, glume extérieure, phycostème.

La glume est composée ordinairement de deux paillettes (fig. K.a.2), ou glumes (froment) rarement d'une seule (fig. K. $b .2$ ). (Ivraie vivace), trèsrarement nulle (fig. K. c.). (Nard élancé, asprelle laux riz).

Les glumes ou paillettes sont inégales ou égales entre elles; chez la plupart des espèces à épi sim- 
ple, les paillettes sont presque égales entre elles (froment, seigle); dans les inflorescences paniculées, elles sont ordinairement très-inégales.
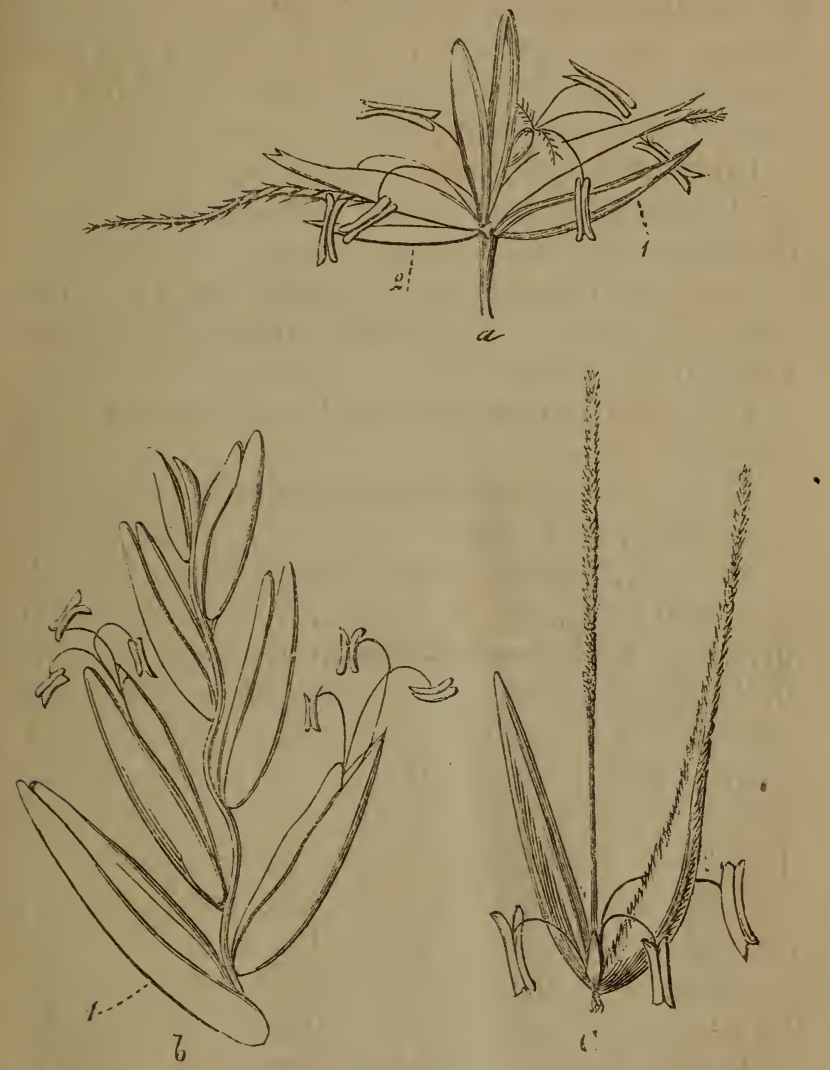

Fig $\mathrm{K}$.

Les paillettes offrent deux faces, l'une interne et l'autre externe, deux bords latéraux et deux extrémités, l'une supérieure, le sommet, et l'autre inférieure ou base. Lorsque la face externe est ar- 
rondie ou bombée on la dit convexe (fig. L 2) elle est plane (fig. L 1) quand une ligne droite peut lui être appliquée; elle est carénée (fig. L 5) lorsqu'une tranche horizontale présente vers le milieu un angle quelconque. La carène peut être aiguë ou aîlée (fig. D 4, 5, 6, ), entière (fig. L 7 ) ou dentée, scabre ou ciliée (fig. L 5, 6, 8).

Eu égard à leur insertion elles peuvent être :

Opposées, lorsqu'elles se trouvent insérées sur la même ligne horizontale. (Seigle, Voir fig. 6. ) Alternes, quand elles sont insérées à des hauteurs différentes, c'est-à-dire l'une plus bas que l'autre (Fig. 29, a.)

Géminées, lorsqu'elles sont insérées par deux, (Fig. 丂̆, orge des prés ).

Eu égard à la manière dont elles se comportent entre elles, on les dit :

Connées, lorsqu'elles sont opposées et soudées à la base (Fig. 52, $a, b, c, d$.) Cette soudure se prolonge quelquefois jusqu'au tiers supérieur de la longueur de la glume. Elle ressemble encore quelquefois à une espèce de petite poche plus ou moins aplatie : jusqu'ici, on n'a pas encore donné à cette forme de nom spécial. Il n'y aurait aucun inconvénient à l'appeler subcuculliforme.

Libres, lorsque les bords ne se recouvrent et ne se touchent pas. (Seigle, fig. 6.)

Engaînantes, quand les bords se recouvrent et se croisent inférieurement. (Froment cultivé.)

Eu égard à leurconfiguration ou figure, on les dit:

Orbiculées, quand la circonférence approche de celle d'un cercle.

Ovales, allongées, arrondies aux deux extrémités, dont l'inférieure est plus large. Cette figure est environ deux fois pluslongue que large. 
Obovales, allongées, arrondies aux deux extrémités, l'extrémité supérieure étant plus large.

Elliptiques, allongées, les deux extrémités égales entre elles, aiguës ou obluses, environ deux fois plus longues que larges.

Oblongues, très-allongées, les deux extrémités égales entre elles; environ trois fois plus longues que larges.

Lancéolées, très-allongées, l'extrémitésupérieure se terminant insensiblement en pointe ; environ quatre fois plus longues que larges.

Linéaires; très-allongées, étroites de mème largeur sur la plus grande partie de leur étendue.

Subulées, très-étroites et diminuant de la base au sommet.

Inéquilatères, quand il y a une nervure médiane qui partage la paillette en deux moitiés inégales.

Eu égard à leur base elles peuvent ètre :

Cordées ou en cœur, lorsqu'elles sont échancrées de facon à présenter deux lobes latéraux arrondis et qu'elles se terminent supérieurement en se rétrécissant.

Eu égard à leur sommet et à leur contour, elles peuvent ètre :

Aiguës, quand elles s'amincissent insensiblement en pointe à leur sommet, qui, circonscrit par deux lignes, représente un angle aigu.

Acuminées, quand elles se rétrécissent brusque- ment et se terminent en une pointe allongée.

Mucronées, quand une pointe vient s'insérer sur le sommet qui est très-obtus.

Obtuses, lorsque leur sommet compris entre deux lignes droites représente un angle ouvert.

(I) Voir les figures de tous ces termes à l'article de la glumelle. 
Tronquées, quand elles se présentent comme si on avait retranché une partie du sommet.

Echancrées, quand le sommet présente uี sinus rentrant en forme de crénelure.

Bifides, fendues au sommet en deux lanières qui n'atteignent pas le milieu de la longeur des paillettes.

Bipartites, quand elles sont fendues jusqu'au delà de la moitié de la hauteur des paillettes.

Entières, lorsqu'elles ne présentent aucune dent ni incision.

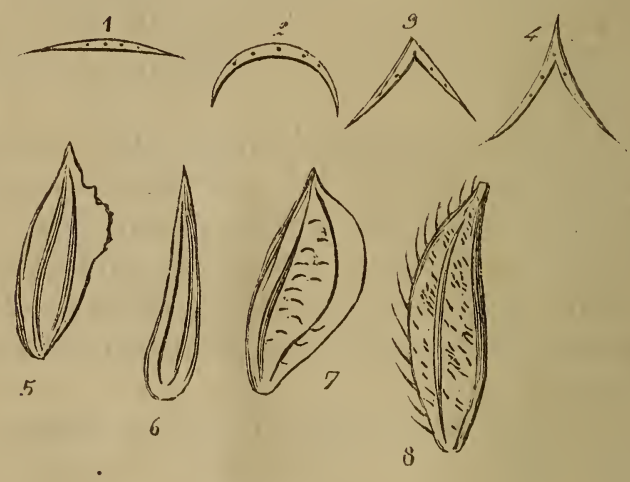

Fig. L.

Dentées, lorsqu'elles présentent des dents bien marquées; il y en a $2, \overline{3}, 4, \breve{5}$, et on les dit alors bidentées, tridentées, quadridentées, elc.

Denticulées, quand les dents sont obscurément marquées et petites.

Erodées, quand les bords sont comme rongés par les insectes (fig. 25).

Pour ce qui concerne les mots mutique e! arjstées, voy. ce qu'on en dit plus loin à l'article Paléole. 
Ces diverses manières d'ètre ne se remarquent pas toujours isolées, mais peuvent se combiner; de là sont venus les termes composés, tels que ovale lancéolé, oblong, oblong-lancéolé, etc.

Eu égard à leur tissu elles peurent ètre :

Membraneuses, lorsqu'elles sont minces, inolles et souples.

Scarieuses, quand elles sont minces, sèches, demi-transparentes.

Roides, lorsqu'elles sont coriaces et résistantes. Herbacées, lorsqu'elics sont vertes, molles et peu résistantes.

Coriaces, quand elles sont épaisses, consistantes et résistantes.

Caronculées, lorsque les paillettes ont pris une forme insolite et se sont épaissies vers le centre de leur plus grande largeur.

Les diverses particularités que nous avons définies à l'article des feuilles s'appliquent aussi à la gelume et à la glumelle.

Les paillettes sont nerviées ou anerves; dans le premier cas, les nervures sont toujours en nombre impair.

La glume est insérée sur un petit calus diciforme (point empâté) qui lui sert de réceptacle.

\section{VI. - du support des flecrons.}

Le support sur lequel s'insèrent les fleurons s'appelle scobine (1); elle est très-courte, presque

(1) I. Dumortier; qui a le premier dénommé l'axe sur lequel s’insèrent les fleurons, admet une scobine et un acicule. Pour lui il y a scobine chaque fois que les fleurons sinsèrent sur une des faces du support; il y a acicule lorsque les fleurs ou les fiemrons s'insèrent sur le culus des ğlumes. L ïdée d'un acicule exclut done nécessairement celle de tout support autre que celni que termine le fleuron.

Chez beaucoup d'espèces, il est très-facile d'appliquer la définition 
nulle ou allongée. Elle est persistante ou fragile, articulée, aciculiforme, bipliée ou en zigzag.

La scobine est persistante, lorsqu'elle ne se divise pas à la maturité en autant d'articles qu'il y a de fleurons. (Eragrostide poilue, fig. 24 a).

La scobine est fragile et caduque, lorsqu'elle se partage en autant d'articles qu'il y a de fleurons (fig. $22 a, b$ ).

La scobine est articulée, lorsqu'elle présente distinctement une série d'angles rentrants et une condensation du tissu au point où le fleuron s'insère (fig. $24 a)$.

La scobine est aciculiforme, quand elle est filiforme et droite. Dans le langage descriptif, on la désigne sous le nom de rudiment pédicelliforme ou aciculiforme ou d'acicule (fig. $39, b$ ).

(Voir plus loin le paragraphe qui traite des rudiments).

La scobine est bipliée, lorsqu'elle est pliée en deux. (Hierchloë boréale, fig. 56. $s$ ).

La scobine est en zigzag, lorsqu elle présente de petites excavations correspondant à chaque fleuron.

La scobine affecte encore quelques autres formes, qu'il est inutile de signaler.

VII. - DE LA GLUMELLE.

Les fleurons sont composés d'une enveloppe ex-

que l'auteur donne de ces organes, mais dans d'autres on est trèsexposé à se fourvoyer; ce n'est souvent qu'à la faveur de l'analogic que présentent ces plantes entr'elles que 11 . Dumor'tier lui-mème a pu elasser certain genre dans ses callillores ou scobiflores. Nous n'en eiterons qu'un exemple c'est celui dı gemre orge, où il existe une scobine. qui est aplatie ou eylindrique et s'insère dans un petit sillon que présente à sa base la paléole interne. N'observe-t-on pas dans l'agrostide épi du vent un pedicelle aciculiforme qui s'insère au même endroit de la palćole interne. Pour ec motif et tant d'autres nous eroyons qu'il est préférable de ne ne point établip la distinction faite par II. Dumortier; elle est d'ailleurs de très-peu d'importanee. 
térieure nommée glumelle. (Voir fig. J. Locuste uniflore et multiflore 2), (corolle, calice, glume extérieure); ils sont nus ou involucellés.

Le fleuron nu est celui qui ne présente à sa base ni poils ni rudiments de fleurons stériles ou avortés. Le fleuron est involucellé, lorsqu'il présente soit à sa base interne ou externe, soit toutà l'entour une collerette de poils (calamagrostide) ou un ou plusieurs rudiments de fleurons avortés, affectant la forme d'écailles glabres, ciliées ou poilues (phalaride). Une locuste de roseau à balais nous fournit un bon exemple de fleuron nu qui est le plus inférieur et de fleurons involucellés placés au-dessus du premier (fig. 25).

La glumelle présente le plus souvent deux écailles florales, (froment) qu'on nomme paléoles (glumelle
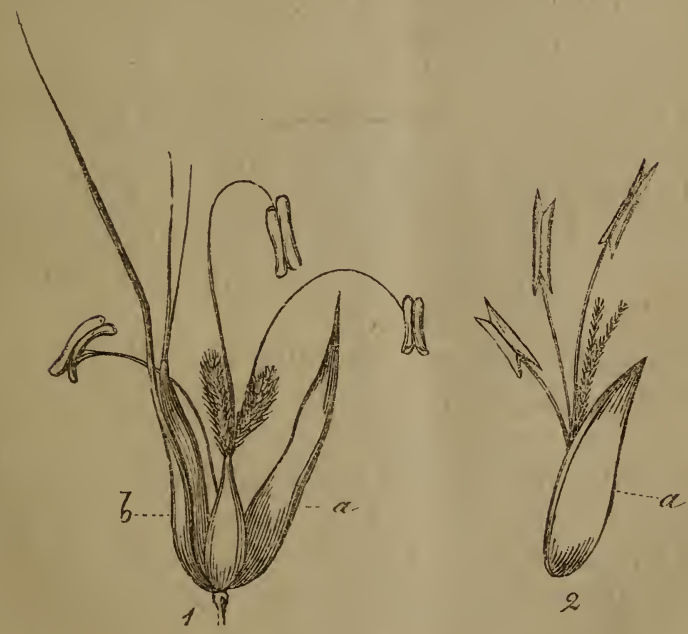

Fig Q.

bipaléolée (fig. 1.a. b.), rarement une seule glumelle (unipaléolée) (fig. Q. 2. a.) (Vulpin). 
On envisage assez généralement la glumelle à une paléole comme le résultat de la soudure des deux paléoles de la glumelle normale.

Les paléoles sont presque constamment inégales et insérées en alternance :l'extérieure, qui est presque toujours l'inférieure, est le plus souvent herbacée et a plus de consistance que l'interne.

La paléole externe est le plus souvent imparinerviée, c'est-à-dire qu'elle présente un nombre impair de nervures; les nombres dominants sont $5, \breve{5}, 7,9$ : dans quelques cas assez rares, l'on en trouve jusquà 15 .

Les paléoles sont :

Arrondies ou convexes, lorsque le dos présente une portion de cercle. Pour vérifier ce caractère l'on prend une tranche horizontale du fleuron, et après l'avoir dégagée de la paléole interne, elle doit présenter une portion de cercle.

Carénées, si la tranche présente un angle vers son milieu. On s'y prend de la même manière que pour démontrer le dos convexe.

La carène peut être nue, lorsqu'elle ne présente aucun appendice membraneux, ou ailée quand elle offre un appendice membraneux; cet appendice peut être entier ou denté.

La paléole externe s'enroule en cylindre autour de l'ovaire et de la paléole interne de manière à former un tour complet; d'autres fois elle n'embrasse que ses bords; d'autres fois, enfin, ses bords restent étendus. Quand elle se replie en ses bords, elle forme ordinairement un angle plus ou moins obtus qui est très-apparent dan certaines espèces.

La paléole externe est sujette à toutes les particularités que nous avons définies à l'article paillettes. 
Comme l'étude de celte paléole est d'une grande importance pour parvenir à la connaissance de certains genres et de beaucoup d'espèces, nous rappellerons les termes qui lui ont été appliqués, en les accompagnant de quelques figures.

Eu égard à sa configuration, on la dit :

Suborbiculée (1) (fig. R. 1), ovale (fig. R. 2), obovale (fig. R. 5), elliptique (fig. R. 4), oblongue (fig. R. 5), lancéolée (fig. R. 6), linéaire (fig. R. 7), subulée (fig. R. 8).
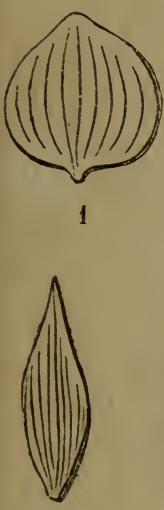

6

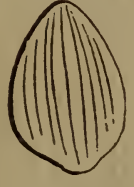

2
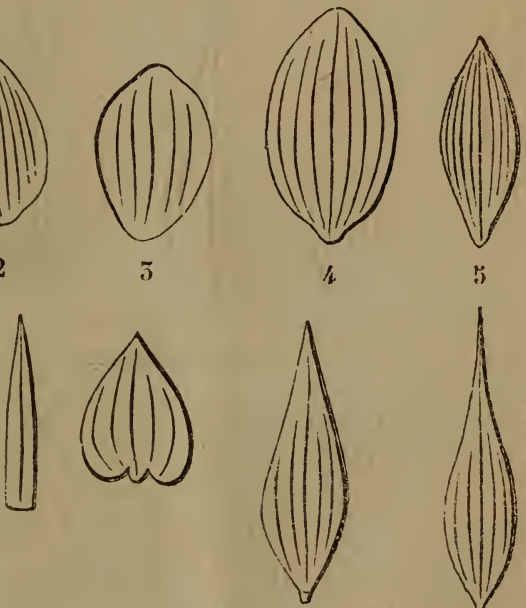

10

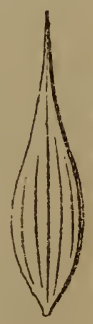

11

Fig. P.

Eu égard à l'échancrure qui existe parfois, elle peut être :

Cordée (fig. R.9).

Eu égard à son sommet et à son contour, elle peut être :

(1) Le mot sub, ajouté aux dénominations, indique que le terme n est pas entièrement applicable.

GRAMINÉES. 
Aiguë (fig. R. 10), acuminée (fig. R. 11), mucronée (fig. S. 1), obtuse (fig. S. 2), tronquée

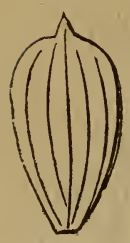

1

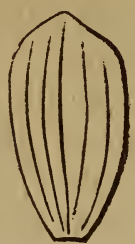

2

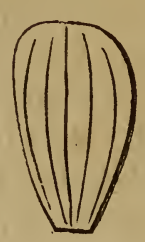

3

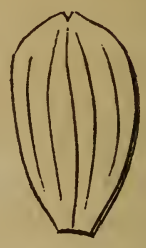

4

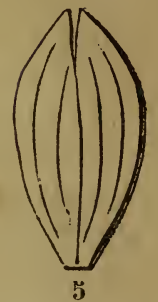

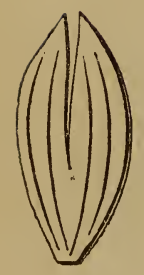

6

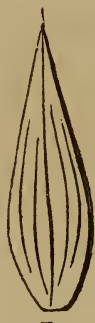

7

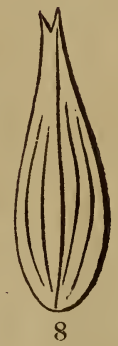

Fig. S.

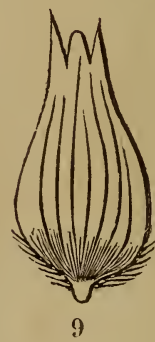

9

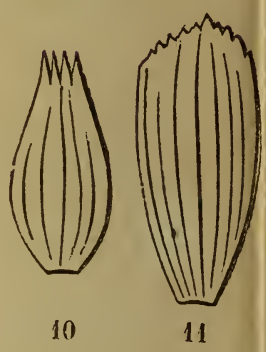

(fig. S. 3 ), échancrée (fig. S. 4), bifide (fig. S. 5), bipartite (fig. S. 6), entière (fig. S. 7), dentée (2, 5, 4 dentée, (fig. S 8, 9, 10), denticulée (fig. S.11).

On dit que la paillette ou la paléole est aristée lorsqu'elle est munie d'appendices allongés, grêles et plus ou moins raides.

A ristée. La paléole peut être diversement aristée; l'arête peut être terminale ou non terminale. L'arête terminale (fig. T. 1, 2, 5 et $4 a$;) n'a pas besoin d'explication, son nom indique assez ce que c'est. L'arête qui n'est pas terminale peut prendre son origine sur tous les points de la hauteur de la paléole; ainsi l'arête peut s'insérer à la base (fig. T s) (arète basilaire); an-dessus de la base, depuis le tiers inférieur (fig. T. 6) (arête épibasi- 
PREMIĖRE PARTIE. - INTRODUCTION.

laire); vers le milieu de sa hauteur (fig. T. 7) (arête dorsale); au-dessus du dos (fig. T. 8 ) (épi-

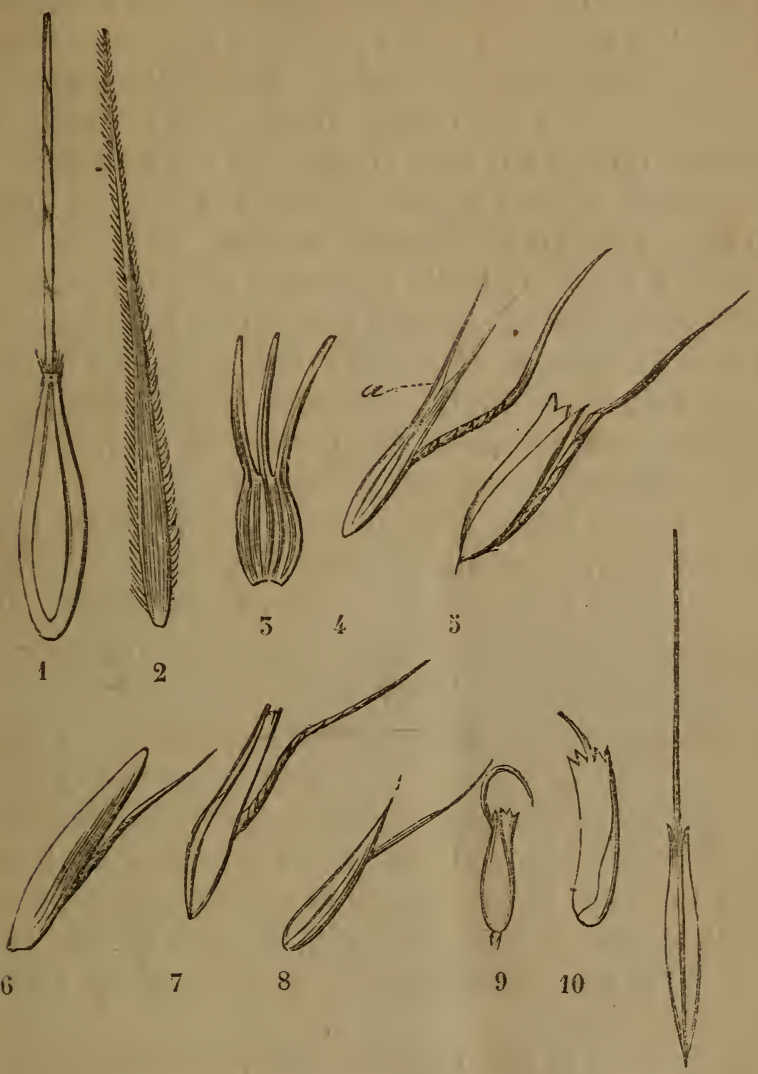

Fiv. T.

11

dorsale); un peu au-dessous du sommet (fig. T.9) (subapicale); dans une échancrure ou émarginure (fig. T. 10, 11) (arète émarginale).

Quelques auteurs, d'aprèsl'illustre agrostographe 
Palisot de Beauvois, qui, le premier, s'est efforcé de débrouiller cette partie difficile de la botanique agricole, ont roulu établir deux catégories dans lesappendices des paillettes et des paléoles, que l'on désigne vulgairement sous le nom de barbe; ils appellent l'une arête et l'autre soie. D'après eux, l'arête est formée d'un tissu dur, coriace, et est insérée subitement et le plus souvent sans une origine apparente; elle servirait aussi souvent, d'après eux, d'étui à la soie qu'elle embrasse et à laquelle elle adhère fortement. La soie n'est qu'un prolongement d'une nervure dont la base fait partie de la substance de la paillette; toujours simple, herbacée, filiforme ou subulée, ordinairement droite, rarement étendue et tournée en spirale.

Si l'on se borne à quelques types, il est facile de faire l'application de la définition; mais s'il s'agit de l'étendre à toutes les espèces, alors cette facilité fait place à la plus profonde indécision : il nous semble donc inutile d'établir cette distinction qui n’a d'ailleurs aucune utilité pratique facilement saisissable. Une paléole sera aristée du moment qu’il existe une pointe plus ou moins allongée, débordant ou dépassant le sommet de la partie membraneuse que présente la paléole de la plupart des espèces; dans le cas contraire, quelque aiguë qu'elle soit, elle n'est pas terminée par une arête et on doit la dire mutique.

L'arète et la soie peuvent être: persistantes, lorsqu'elles nese détachent pas des enveloppes florales à la maturité du grain; caduques, quand elles s'en détachent avant ou à la maturité du grain; droites (fig. U. 1), lorsque les fibres qui la composent se rapprochent de cette ligne; tordues (fig. U. 2), lorsque les fibres ont subi une torsion plus ou moins 
forte sur elles-mêmes : cette torsion est très-apparente dans beaucoup de cas; genouillées (fig. U. 3), lorsqu'elles semblent avoir subi un commencement

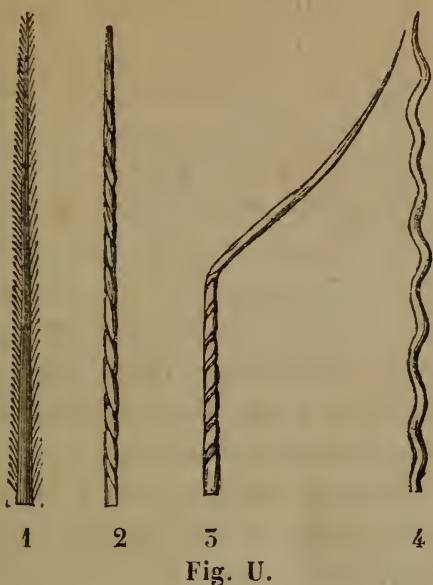

Fig. U.

de torsion et qu'elles dévient de la perpendiculaire en formant un angle plus ou moins ouvert ; flexueuses (fig. U. 4), lorsqu'elles décrivent des zigzags ou diverses inflexions obtuses.

La paléole interne ou supérieure est ordinairement de nature membraneuse, mince, aplatie et comprimée; les deux bords latéraux sont plus ou moins repliés en dedans, de façon à présenter une rainure dans laquelle l'ovaire se trouve logé et s'allonge en mûrissant. Elle est presque toujours bicarénée et parinerviée dans les locustes multiflores, et imparinerviẻe lorsqu'il n'existe qu'un seul fleuron, sans aucun rudiment de fleuron stérile.

\section{VIII. - DE la gLemellule.}

La plupart des graminées, sinon toutes, indépendamment de la glume et de la glumelle, offrent en- 
core une ou plusieurs écailles qu'on trouve à la base de l'ovaire et en dehors des étamines; ces écailles ont été désignées sous le nom de glumellule, nectaires, écailles, lodicule, corolle. La glumellule est presque toujours insérée du côté de la paléole externe et se compose de deux (fig. V, 1) ou

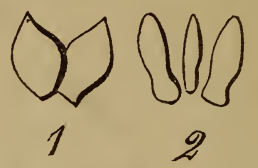

Fig. V.

trois (fig. V. 2) paléolules; elles sont quelquefois soudées entre elles, mais le plus communément libres, glabres, velues ou ciliées. Leur forme est assez variable : dans quelques cas rares, la glumellule dépasse l'ovaire. (Ammophile des sables. Stipe capillaire.)

\section{IX. - des Rudments.}

Certaines espèces à locustes uniflores présentent entre la glume et la glumelle un ou deux petits organes, vestiges de fleurons stériles ou de scobine; on leur a imposé le nom de rudiments. Ils se présentent tantôt sous la forme de paléole (rudiment paléoliforme (fig. J. 1), comme dans l'alpiste

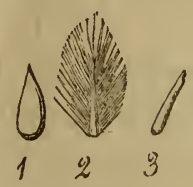

Fig. J.

des Camaries; tantôt sous celle d'une petile membrane fortement cilićc ou velue (rudiment péni- 
cilliforme, fig. J. 2), comme l'alpiste roseau. Quelques agrostographes ont encore considéré comme un rudiment de fleuron un organe qui a la forme d'un pédicelle et qu'on trouve quelquefois à la base externe de la paléole interne, et l'ont appelé rudiment pédicelliforme. Cette désignation, eu égard à l'origine de cet organe, n'est pas exacte, attendu qu'il correspond à la scobine des locustes pauci ou multiflores; ce qui appert du petit renflement, véritable rudiment de fleuron, dont il est parfois muni à son sommet. Quoi qu'il en soit, nous le désignons sous le nom de rudiment aciculiforme (fig. J. 5), qui peut être atténué ou subclaviforme; ou bien ce n'est qu'une scobine aciculiforme, nue ou terminée par un rudiment de fleuron allongé ou oblong, rarement arrondi. Elle se rencontre presque exclusivement dans les locustes qui ne présentent qu'un seul fleuron fertile.

\section{X. - des organes sexuels.}

Lesorganes sexuels, comme dans toutes les fleurs complètes, sont les étamines et le pistil.

Les étamines sont insérées sur le réceptacle du fleuron; le nombre en est assez peu variable. On en trouve généralement trois, quelquefois deux, rarement une, six ou huit. Les étamines sont composées du filet et de l'anthère. Les filets (fig. W. $b .7$ ) sont capillaires plus ou moins longs et portent des anthères (fig. W. $a . b .6$ ) terminales biloculaires, bifurquées au sommet et à la base. Elles sont nues ou barbues. Elles s'ourrent latéralement et dans le sens de leur largeur. Le pollen ou poussière fécondante varie dans sa couleur : tantôt il est blanchâtre, d'autres fois jaunâtre; enfin il est aussi 
orangé ou violet. Cette couleur n'est pas toujours la même dans tous les individus d'une même espèce : elle a servi à établir quelques variétés.

Le pistil est composé de l'ovaire, des styles et des stigmates.

L'ovaire (fig. W. $b .5$ ) est globuleux, turbiné ou allongé, uniloculaire, uniovulé, à ovule adhérant à la paroi interne.

Les styles (fig. W. b. 4.) sont ordinairement au nombre de deux, rarement un seul, plus ou moins allongés, terminaux ou latéraux.

Les stigmates (fig. W. $b .5$ ), qui avec l'ovaire forment les deux parties essentielles du pistil, sont presque constamment au nombre de deux, simplement pubescents ou plumeux. Le maïs et le nard, parmi les espèces cultivées ou indigènes, n'en présentent qu'un.

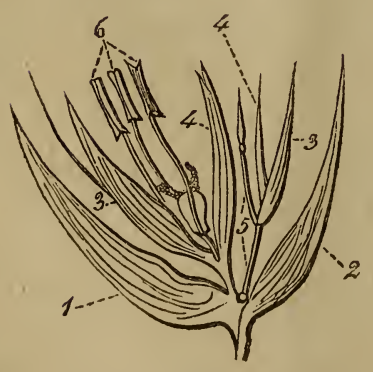

a

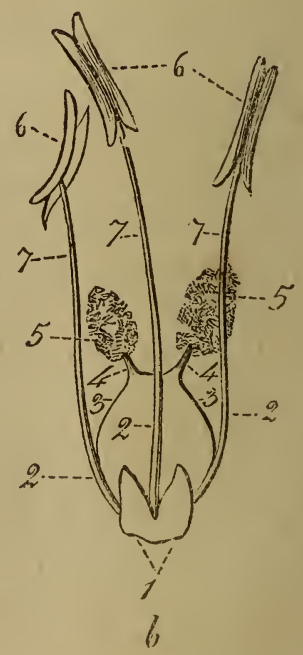

Yig. IV. 
Les stigmates sortent au sommet du fleuron vers le milieu ou vers la base (1).

\section{XI. - du FrutT.}

Le fruit est un caryopse ou grain fréquemment sillonné ou canaliculé; sa forme varie beaucoup : combinée avec l'absence ou la présence du sillon ou canalicule, elle a servi à l'illustre Reichenbach pour établir ses groupes naturels.

Le grain est presque entièrement composé d'un périsperme farineux dont la consistance est loin d'être toujours la même. Tantôt il est dur et corné (riz, maïs, froment de Pologne); d'autres fois il est assez tendre et se laisse écraser sous la dent (froment cultivé); enfin, il est aussimou et prend toutes les formes qu'on veut lui donner : le lagurier, l'a. voine pubescente sont dans ce cas.

A la partie inférieure de la graine, le périsperme présente une excavation ou dépression où l'on trouve l'embryon.

La partie latérale élargie qui s'appuie sur le périsperme est l'hypoblaste de Richard et le cotylédon vrai des embryogénistes; la gemmule lui est accolée et est recouverte par une gaine qui est le coléoptile de Mirbel et la vaginule d'autres auteurs, et pour nous une feuille primordiale réduite à sa gaine, comme on en trouve dans tous les hourgeons des graminées, quel que soit l'endroit où ils se développent. Le mamelon qui le termine inférieurement renferme une ou plusieurs radicelles qui lors

(1) Fig. W. $a$, Locuste d'avoine cultivée; 1 paillctle externe ; 2 pailletle interne; $\bar{J}$ paléoles externes; 4 paléoles internes; 5 scobine; 6 étamines. $-b 1$ paléolulés; 2 étamines; 3 ovaire; 4 styles; 5 stigmates; 6 anthères, 7 filets. 
de la germination se dégagent des couches externes épaissies; celles - ci persistent pendant quelque temps autour d'elles sous la forme d'une collerette qui n'existe pas chez toutes et que l'on appelle coléorhize. 


\section{DEUXIÈME PARTIE.}

\section{ANALYSES ET DESCRIPTIONS.}

AN.ALYSE DES TRIBUS.

Deux modes d'inflorescence sur la même plante; épis axillaires et panicule simple terminale ou épi composé. Maydées (I).

Jamais deux modes d'inflorescence sur la même plante. . . . . . . . .

Axe primaire de l'inflorescence simple (épi simple) très-rarement rameux, présentant sur une face ou sur deux faces presque opposées des

2 dépressions ou des excavations correspondant aux locustes ou rachis en zigzag, articulé et denté ...... Truticacées (II).

Plantes ne réunissant pas ces caractères . . . . . . . . . . . . .

Locustes à deux ou plusieurs fleu5 rons fertiles (sauf deux espèces du

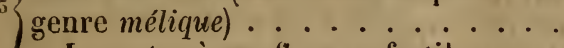

Locustes à un fleuron fertile. . . .

Une paléole au moins de chaque locuste, munie d'une arête basilaire, dorsale ou épidorsale, qui est tortillée

4 ou genouillée. . . . . . . . Avénacées (iv).

Paléole ne présentant pas d'arête basilaire dorsale ou épidorsale qui soit tortillée ou genouillée . . . . . . Festucacées (III).

Locuste à deux ou trois fleurons développés dont un hermaphrodite et un ou deux mâles, ou locuste renfermant deux paléoles aristées au dos, à la base ou au-dessous du sommet. . Arriénathéracées

Locustes ne contenant qu'un fleuron développé, parfois accompagné d'un ou de deux rudiments ....... 6 ou 13. 
Paillettes carénées ou non carénées, mais dans ce dernier cas plumeuses ou aristées au-dessous du sommet, ou glume nulle. . . . . . . . . . .

Paillettes planes arrondies ou convexes, ni plumeuses, ni aristées audessous du sommet; une glume à une ou deux paillettes .........

7) Panicule simple digitée ..... Cynodonées (XI).

\{ Pas de panicule simple digitée . . . 8.

8f Glume nulle . . . . . . . Phalaridacées (x).

Une glume. . . . . . . . . . 9 .

Panicule spiciforme compacte ou épi cylindrique ou filiforme à fleuron nu ou accompagné d'un ou de deux rudiments paléoliformes ou pédicelliformes, ou bien panicule presque lobée à paillettes aristées au-dessous du sommet, ou bien enfin panicule rameuse presque lobée à fleuronsfertiles accompagnés de deux rudiments pénicilliformes; paillettes jamais très-allongées, étroites, plumeuses . . . . Panicule rameuse, lobée ou étalée, à fleuron dépourvu de tout rudiment quelconque ou accompagné d'un seul rudiment pédicelliforme ou pénicilliforme, ou bien panicule spiciforme compacte à fleuron fertile accompagné d'un seul rudiment pédicelliforme ou subpénicilliforme, et dans ce dernier cas paillettes allongées, étroites, plumeuses. . . . . . . . . . Agrostidicées(vi).

Paléole externe s'enroulant en cylindre autour de l'ovaire; trois paléo-

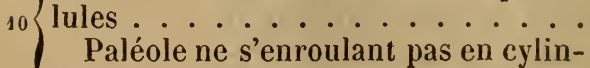
dre autour del'ovaire; deux paléolules.

Phalaridacérs $(\mathrm{x})$.

Stipacées (vii).

11.

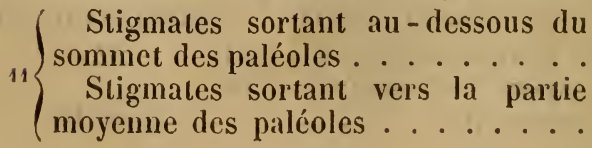

Panicacéres (xi).

12. 
DEUXIÈyE PARTIE. - ANALYSES ET DESCRIPTIONS. 55

Panicule simple digitée ou subdi-

12 Oanicule rameuse, à rameaux inférieurs disposés par $4-10 \ldots \ldots$ MILIaGÉEs (VIII).

Stigmates sortant au sommet du fleuronà paléoles carénées, très-rarement vers le tiers supérieur on inclus (1); on bien les paillettes sont à peine caré-

15 . nées et longuement aristées au-dessous du sommet (2); panicule non digitée. Phalaridacées (x).

Stigmates sortant vers le tiers inférieur, vers le tiers supérieur ou vers la partie movenne du fleuron. .... 14 ,

Paillettes carénées ou plumeuses. . ${ }_{14}\left\{\begin{array}{c}\text { Paillettes à dos un peu arrondi où } \\ \text { convexe . . . . . . . . }\end{array}\right.$

Stigmates sortant un peu au-dessous du'sommet du fleuron; paniculedigitée. CrnodonéES (IX).

Stigmates sortant vers la partie

moyenne des paléoles; panicule non digitée....................

Stigmates sortant vers la partie inférieure ou la partie moyenne des paléoles. . . . . . . . . . . .

Stigmates sortant au sommet ou un 17. peu au-dessous du sommet des paléoles................. Pantcacées (vi).

${ }_{17}\{$ Trois paléolules . . . . . . Stipackées (VII).

${ }^{17}\{$ Deux paléolules. . . . . . 16.

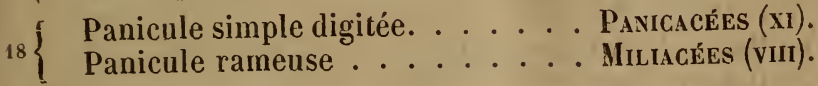

(1) Le genre asprelle fait l'objet de cettc exception ; lantòt ses stigmates se font jour, d'autres fois ils restent inclus.

(2) Le genre polypogon présente une particularité non moins remarquable que l'asprelle : c'est que tantôt ses stigmates sortent au sommet du fleuron, tantôt vers son tiers supérieur, et tantòt enfin ils restent inclus. Par l'ensemblè de leurs caractères, ces genres se rapprochent beaucoup des agrostidicées.

Nous ne doutons ancunement qu'ils n'y soient mieux à leur place. 
ANALYSE DES GENRES.

Ire tribu. - Fiagalées.

\section{.......................... MIIS (1).}

He tribu. - Truticacées.

Deux ou trois locustes sur chaque

${ }_{1}$ dent du rachis ..........

1 Une locuste sur chaque dent du rachis.............

Locustes hermaphrodites uniflores

à arêtes nulles ou à peine deux ou

2 trois fois plus longues que le fleuron.

Plantes vivaces. . . . . . . Elyme (2).

Locustes uniflores ........ ORGE (כ).

3 Glume nulle, épi unilatéral. . . . NARD (9).

3 Une glume, épi distique . . . . . 4 .

Paillettes munies de 2 à 5 arêtes. . OEgroope (6).

4) Paillettes mutiques ou munies d'une

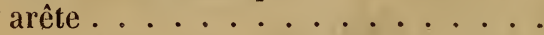

(Locustes présentant le dos au rachis. Ivrare (7).

: $\{$ Locustes présentant une des faces

latérales au rachis. Glumes à deux

paillettes............ 6 .

Locustes à unfleuron fertile, accom-

6) pagné d'un rudiment ................ Lepiure (8).

$6\{$ Locustes bi ou multiflores, l'un des

(lleurons mâle ou neutre. . . . . . 7 .

f) Paillettes linéaires-subulées . . Sergle (4).

7 Paillettes lancéolées, ovales ou

coblongues ............ Froment (5).

ORDRE DE SUCCESSION DES GENRES.

2. Elyme (Elymus L.); 3. Orge (Hordeum L.); 4. Seigle (Secale L.); 5. Froment (Triticum L.); 6. OEgylope (OEgylops L.); 7 Ivraie (Lolium L.); 8. Lepiure (Lepiurus Dmtr.); 9. Nard (Nardus L.). 
DEUXIÈME PARTIE. - ANALYSES ET DESCRIPTIONS. 55

III tribu. - Festucucées.

Locustes fertiles entremêlées de locustes stériles réduites à des bractées pectinées ou pinnées ...... Grvosure (10).

Locustes fertiles non entremêlées de locustes stériles réduites à des bractées pectinées........

Locustes de deux à six fleurons, l'inférieur mâle, glabre; les autres munis d'un involucelle de longs poils soyeux. Roseau (22).

Plantes ne réunissant pas ces caractères.

Paléole externe comprimée latéra-

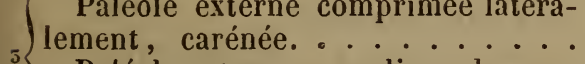
5.

Paléole externe arrondie au dos, non carénée.

Panicule unilatérale à rameaux étalés ou resserrés; locustes recourbéesconcaves, ramassées en glomerules unilatéraux compactes; paléole externe courtement aristée. ...... DaGtyle (18).

Panicule étalée ou spiciforme, non formée de locustes recourbées - concaves, ramassées en glomerules unilatéraux. ..........

Panicule spiciforme plus ou moins compacte ; glume très-large, enveloppant presque complétement la locuste.

Panicule plus ou moins étalée. .

Stigmates filiformes sortant au sommet des paléoles. ......... Seslerie (26).

Stigmates plumeux sortant vers la (base des paléoles. ........ Koelerie (25).

Feuilles caulinaires à limbes trèscourts; gaîne non fendue . . . . . Seslerie (26).

Feuilles caulinaires à limbes plus ou moins longs; gaîne fendue. . . . . Koelerie (2כ̃.)

Ligule membraneuse; scobine fra8 gile.

6 ou 7.

8.

Ligule pileuse; scobine persistante. Eragrostide (21). 
$9\{$ Paillettes érodées. . . . . . . Catabrose (20).

Paillettes entières. . . . . . Paturin (19).

Chaume ne portant que deux ou quatre feuilles qui s'insèrent toutes à la base sur des noeuds très-rapprochés, la gaîne de la feuille inférieure recouvrant les nœuds et les gaînes des autres feuilles; locustes à deux ou trois fleurons inférieurs fertiles, les autres stériles. . . . . . . . . . . Enodie (13).

Plantes ne réunissant pas ces caractères. . . . . . . . .

( Glume enveloppant les locustes; paléole externe à trois dents; panicule 11 simple de quatre à douze locustes . . Triodre (24). Plantes ne réunissant pas ces caractères. . . . . . . . . . .

$12\left\{\begin{array}{c}\text { Paléole externe comprimée-convexe, } \\ \text { cordée à la base, arrondie au sommet, } \\ \text { mutique. . . . . . . . . . . . . Brize }(14) \\ \text { Paléole non cordée à la base. . }\end{array}\right.$ Brize (14).

(Épi composé , compacte ; ligule ${ }_{15}$ membraneuse, courte, fortement ci-

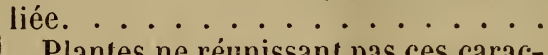
teres. ne reunissant pas ces caracAelbroechie (17).

14.

Panicule plus ou moins unilatérale (et spiciforme ou étalée; glume longue, embrassant presque la locuste composée d'un ou de deux fleurons fertiles 14 accompagnés d'un ou de deux flenrons supérieurs stériles; paléole externe mutique. ............

Plantes ne réunissant pas ces caractères.

MÉliQue (23).

15.

Stigmates s'insérant latéralement

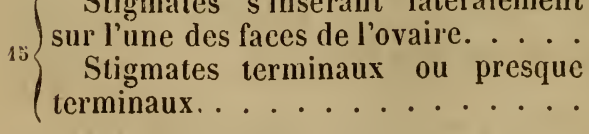
Brome (12). 
DEUXIĖUE PARTIE. - ANALISES ET DESCRIPTIONS. 57

nure.
nurée, et mucronée dans l'émargi-

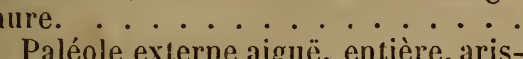

Paléole externe aiguë, entière, aris-

tée, rarement mutique. . . . . . . Fétuque (11).

Paléole externe mucronée dans l'émarginure ou un peu au-dessous de l'émarginure ; panicule unilatérale ${ }_{17}$ roide. . . . . . . . . . ScLerochlö (16).

Paléole externe obtuse entière ou denticulée; panicule régulière ou étalée, ou unilatérale penchée. . . . . Gu GcÉrie (I亏).

\section{7.}

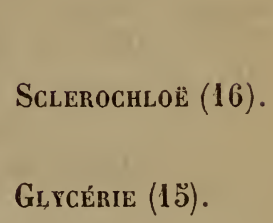

\section{ORDRE DES GENRES.}

10. Cynosure. (Cynosurus L.)

11. Fétuque. (Festuca L.)

12. Brome. (Bromus L.)

13. Enodie. (Enodium P. D. B.)

14. Brize. (Briza L.)

15. Glycérie. (Glyceria R. Br.)

16. Sclerochloë. (Sclerochloa P. D. B.)

17. Aelbroeckie. (Aelbroeckia N.)

18. Dactyle. (Dactylis L.)

19. Paturin. (Poa L.)

20. Catabrose. (Catabrosa P. D.B.)

21. Eragrostide. (Eragrostis P. D. B.)

22. Roseau. (Arundo L.)

23. Mélique. (Melica L.)

24. Triodie. (Triodia P. D. B.)

25. Koelerie. (Koeleria)

26. Seslerie. (Sesleria Ard.)

\section{IVe tribu, - Avéneacées.}

( Paléole externe munie d'une arête épi-basilaire, articulée vers le milieu

1 et renflée en massue vers le sommet. Convá́phore (27).

Plantes ne présentant pas une telle arête. 
Paléole externe irrégulièrement tronquée, à trois, quatre ou cinq dents, et munie d'une arête basilaire ou épi-basilaire. . . . . . . . Canche (29).

2 Paléole externe du fleuron infé. rieur bidentée, bifide ou biaristée au sommet, munie d'une arête insérée vers le milieu de sa hauteur ou plus haut. . . . . . . . . . Avorne (28).

ORDRE DE SUCCESSION DES GENRES.

27. Corynephore. (Corynephorus

P. D. B.)

28. Avoine (Avena L.)

29. Canche. (Aira L.)

ve tribu. - Ap»hénathérées.

(caillette externe de moitié plus courte que l'interne; deux paléoles aristées dans chaquelocuste, dont l'une

genouillée; étamines $2 \ldots \ldots$ Flouve (33).

Plantes ne réunissant pas ces caractères. . . . . . . . . . .

Locustes triflores; fleuron supérieur (hermaphrodite à deux étamines; les

2 latéraux mâles à trois élamines. . . HIErochloE (32).

Locustes biflores dont un fleuron mâle et l'autre hermaphrodite. . . . 3.

3) Fleuron hermaphrodite supérieur.ArRuÉnathéRÉEs(30)

Fleuron hermaphrodite inférieur. . HoulQue (31).

ORDRE DE SUCCESSION.

30. Arrhénathère. ( Arrhenathcrum P. D. B.)

31. Houlque. (Holcus L.)

32. Hierochloê. (Hicrochloa Gmel.)

35. Flouve. (Anthoxanthum L.) 
DEUXIÈHE PARTIE. - ANALYSES ET DESCRIPTIONS. ¿99

VI tribu. - Agrostidicées.

1 Panicule spiciforme compacte. .

Panicule plus ou moins étalée. . .

2.

4.

Paillettes non distinctement carénées, mais ciliées, velues, comme plumeuses; paléoles bifides, biaristées au sommet et munies d'une arête dorsale et tordue à la base. . . . . . Lagurier (36).

Paillettes fortement carénées; paléole externe munie d'une arête courte subterminale. . . . . . . Ammophile (37).

Paléoles entourées à la base d'un involucelle de poils soyeux plus ou moins longs, ou fleuron fertile accompagné d'un rudiment fortement velu, cilié. Calanagrostide

Paléoles glabres ou munies à la base de faisceaux de poils extrêmement courts, ou fleuron fertile accompagné d'un pédicelle non cilié. . . . . Agrostỉe (35).

ORDRE DE SUCGESSION.

34. Calamagrostide. (Calamagrostis R.)

5ว. Agrostide. (Agrostis L.)

36. Lagurier. (Lagurus L.)

57. Ammophile. (Ammophila Host.)

VII' tribu. - Stipacées.

Paléole externe entière, munie d'une arête terminale longue fortement tordue, caduque. ......... Strpe (38).

Paléole externe plus ou moins sensiblementbidentée au sommet, ou entière.............2 2 ou 5 .

Paléole externe velue-ciliée, munic d'une arête persistante. . . . . . Lasiagrostide (59)

$2\{$ Palćole externe glabre, munie d'une arête caduque. . . . . . Piptathène (40). 


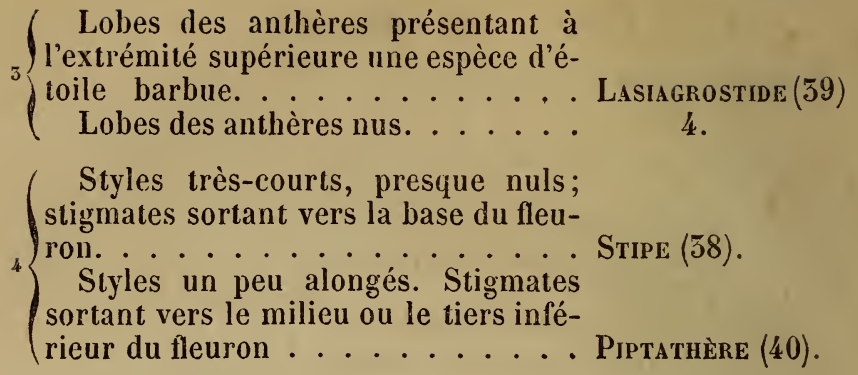

ORDRE DE SUGCESSION DES GENRES.

38. Stipe. (Stipa L.)

39. Lasiagrostide. (Lasiagrostis LK.)

40. Piptathère. (Piptatherum P. D. B.)

WIIT trilbu. - Mfilicées.

41. Milier. (Milium L.).

IX trilbu. - Cynodocées.

Chiendent (42)

42. Chiendent. (Cynodon Rich.)

x tribu. - Protarialacées.

G Glume nulle; panicule étaléc. . . Asprelle (45).

1 Une glume; panicule spiciforme on

Cétalée. .............. 2.

Paillettes obtuses, subémarginées

2 ou subtronquées, munies chacune

d'une arête. .............. Polrogon (46).

Paillettes mutiques. . . . . . . . 5. 
DEUXIÈME PARTIE. - ANALYSES ET DESCRIPTIONS. 61

Locustes contenant un fleuron fertile accompagné d'un ou de denx rudiments pénicilliformes ou paléoli-

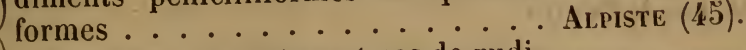

Locustes ne contenant pas de rudiments pénicilliformes ni paléoliformes. .........

Paléole unique munie d'une arête + dorsale ou hypodorsale . . . . . . Vulpis (47).

Deux paléoles mutiques .....

Épi filiforme presque unilatéral; paléoles tronquées, velues-ciliées. . Mirore (44).

Épi ou panicule spiciforme régulier, cylindrique, ovale ou ovale-oblong. . FuÉole (46).

ORDRE DE SUCCESSION.

43. Asprelle. (Asprella Schub.)

44. Mibore. (Mibora Adans.)

45. Alpiste. (Phalaris L.)

46. Fléole. (Phleum L.)

47. Vulpin. (Alopecurus L.)

48. Polypogon. (Desf.)

Xle tribu. - Panicacées.

Locustes géminées, l'une herma$\{$ phrodite et sessile, l'autre pédicellée et mâle ou neutre .

Plantes ne réunissant pas ces caractères.............

5.

$2\{$ Panicule simple digitée .... Barbon $(56)$.

Panicule rameuse ...... Sorghier (55).

Paillette extérieure à sept nervures

Shargées d'aspérités crochues . . . Tragier (49).

3 Paillette externe nulle, glabre ou scabre, pubescente ou ciliée .... 
Glume environ trois fois plus longue

que le fleuron qui est aristé. . . . Gastridie (50).

Pas de glume environ trois fois plus longue que le fleuron ....... 5 .

Locustes fertiles entremêlées de locustes stériles réduites à des soies ou pédicelles denticulés, souvent

assez longs et plus ou moins épais : . Sétaire (51).

Pas de locustes fertiles entremêlées de locustes stériles réduites à des soies denticulées.

6.

Panicule rameuse, à locustes plus

s ou moins longuement pédonculées . P Panis (54).

Panicule simple ou presque simple. 7.

Panicule ailée ou subailée; paléole externe du fleuron fertile pourvue au sommet d'une pointe aristiforme plus

i ou moins allongée . : . . . Oplisuène (53).

Panicule simple, digitée ou subdigitée, formée de $2-15$ divisions; paléole externe du fleuron fertile mutique............ Digitarke (52).

ORDRE DE SUCCESSION DES GENRES.

49. Tragier. (Tragus Desf.)

50. Gastridier. (Gastridium P. D. B.)

51. Setaire. (Setaria P. D. B.)

52. Digitaire. (Digitaria Scop.)

53. Oplismène. (Oplismenus Dmtr.)

54. Panic. (Panicum L.)

5๊. Sorghier. (Sorghum M.)

56. Barbon. (Andropogon L.) 
DEUXIĖye PARTIE. - ANALYSES ET DESCRIPTIONS. 65

Ire tribu. - Draydées.

Les maydées présentent deux modes d'inflorescence : l'une en panicule et l'autre en épi spadiciforme. La panicule est simple, terminale et composée de locustes màles; les locustes femelles sont disposées en épi et s'insèrent dans des excavations d'un axe épais, cellulo-charnu. Ces épis sont étroitement renfermés dans des bractées engainantes. 


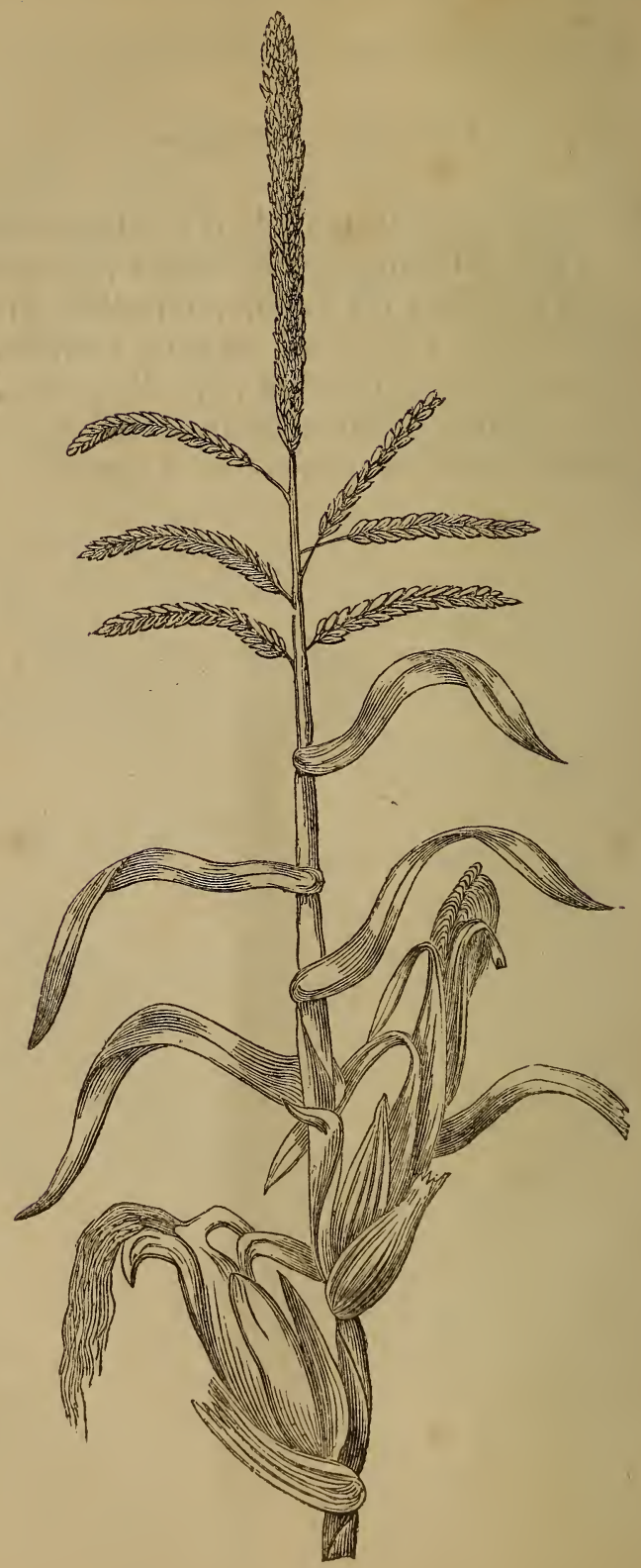


DEUXIĖyE P.RTIE. - ANALYSES ET DESCRIPTIONS. 63

GENRE 1. - MAÏs.

Inflorescences unisexuelles : locustes màles, géminées, biflores; disposées en une panicule terminale, fleurons sessiles; glume à deux paillettes lancéolées, coriaces, arrondies, mutiques; paléoles égales, lancéolées, émarginées, mutiques; paléolules 2, épaisses, tronquées, conniventes et libres; étamines $\overline{5}$; filets longs.

Locustes femelles disposées en gros épis axillaires irrégulièrement polygones, recouverts par un certain nombre de bractées spathiformes; épis composés d'un axe cellulaire charnu, épais, offrant de quatre à treize faces longitudinales et portant chacune une double rangée de locustes sessiles et géminées, implantées dans des excavations; chaque locuste contient un fleuron fertile et un fleuron inférieur neutre; glume à deux paillettes, mutiques, larges, membraneuses, dépourvues de nervures.

Fleuron fertile. Deux paléoles larges, étiolées, convexes, obtuses, légèrement échancrées, anerves; rarement des paléolules et des vestiges d'étamines.

Fleuron neutre. Deux paléoles larges, l'inférieure membraneuse, un peu gibbeuse à la base, étendue ou à peine involutée en ses bords; la supérieure membraneuse, un peu épaissie vers le bas, subcuculliforme, aplatie; glumellule à deux paléolules distinctes, mais très-courtes; ovaire nul, rudimentaire, très-rarement fertile.

Ovaire glabre, subglobuleux, portant un style terminal, indivis, qui se confond avec un stigmate filiforme velu ayant cinq à six pouces de longueur, pendant. Grain anguleux, subréniforme, coloré, 
luisant, entouré à sa base par les paillettes et les paléoles qui sont persistantes. Locustes supérieures très-rarement mâles ou hermaphrodites.

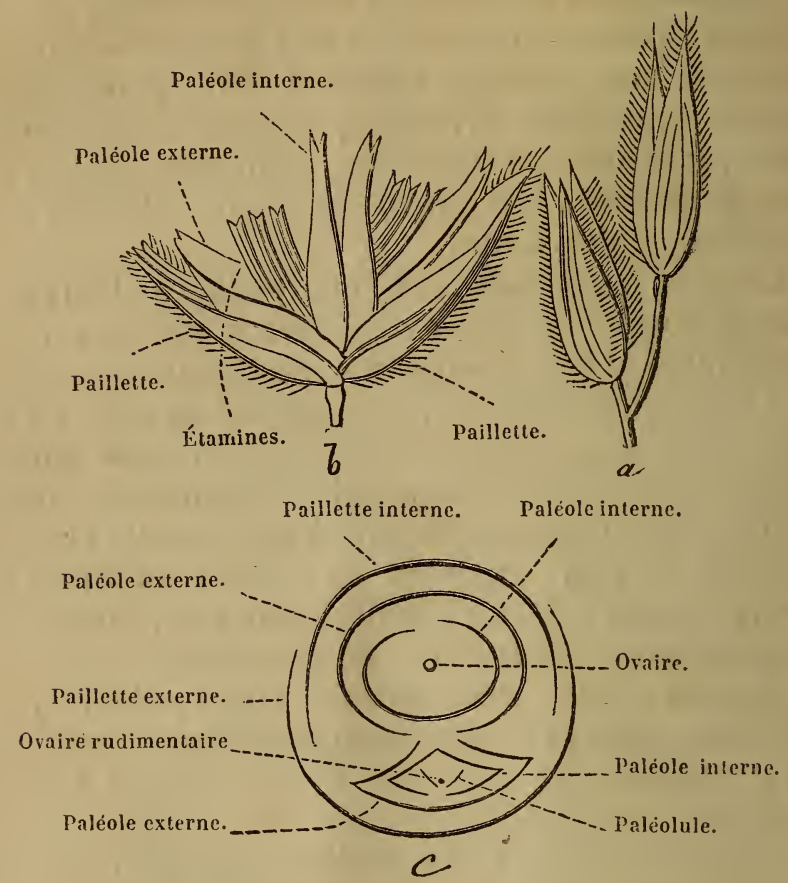

Fig. 2 (1).

MAÏS CULTIVÉ (MAYS SATIVA. TOURN.; ZEA MAYS L.)

Le maïs présente des chaumes forts, droits, articulés, qui s'élèvent jusqu'à deux mètres de hauteur et qu'embrassent de grandes et larges feuilles glauques et coriaces fortement nervées; ligule

(1) a Locustes géminées, mâles. 6 locuste mâle qui fait voir les principaux caractêres. $c$ Diagramme de la locuste femelle. 
courte. La panicule des fleurs mâles est terminale, large, étalée; les locustes femelles sont disposées en épis axillaires qu'enveloppent un certain nombre de bractées larges, pourvues d'une partie de limbes foliacés, au sommet desquels on voit sortir à la floraison des stigmates filiformes qui forment une belle touffe retombant vers la terre; les

- bractées ne s'entr'ourrent qu'à mesure que la maturité approche.

Cette plante qui fleurit en août est annuelle.

On ne connaît qu'une seule espèce de maïs. C'est le zea mays de Linné; mais on en cultive plusieurs variétés que nous allons analyser.

\section{ANALYSE DES ESPÈCES.}

Grain subspatuliforme, petit, presque transparent, muni d'un bec extrê1 mement court. . M. A PERLE.

Grain ne réunissant pas ces caractères.

2 Grain terminé par un bec recourbé. M. A BEc.

Grain terminé par un bec. . . 5.

Série longitudinale de vingt-quatre

à soixante grains. ..........

Série longitudinale de vingt à vingt et

(un grains; huit à seize rangées. . . . M. A PoUlet.

Quarante-cinq à soixante grains;

$4\{$ huit à dix rangées. ..........

Vingt-quatre à quarante grains. . .

5.

6.

¡) Six à huit rangées. . . . . . . . M. DE Virginie.

Huit à dix rangées. . . . . . . . M. de Pensilvanie.

Vingt-quatre à vingt-huit grains; (6) huit à dix rangées. . . . . . . . . . Trente à quarante grains. . . . . .

Trente à trente-cinq grains; douze

$\{$ à quatorze rangées. . . . . . . . M. D'ÉTÉ.

Trente-cinq à quarante grains; dix (à douze rangées. . . . . . . . . . M. D'Automne. 
Ces diverses variétés sont cultivées les unes comme plantes fourragères, les autres comme céréales.

Le maïs d'été s’élève à un mètre et demi; le maïs d'automne à deux mètres; le maïs quarantain d'un demi-mètre à un mètre; le maïs nain et le maïs à bec à un demi-mètre environ; le maïs de Pensylvanie et le maïs de Virginie de deux à trois mètres; le maïs à perle atteint deux mètres de hauteur ou plus et se ramifie beaucoup.

Le grain de ces diverses variétés est jaune, blanc et varié.

\section{Ire tribu. - Trilicacées.}

Les triticacées offrent ou bien un axe primaire dinflorescence très-allongé qui présente sur une face ou sur deux faces presque opposées des dépressions ou des excavations correspondant aux locustes, ou bien un axe primaire en zigzag, articulé et denté; les locustes sont sessiles ou subsessiles, à un, deux ou plusieurs fleurons fertiles; la glume est ordinairement scobifère, rarement callifère. 
DEUXIĖHE PARTIE. - ANALYSES ET DESCRIPTIONS. 69

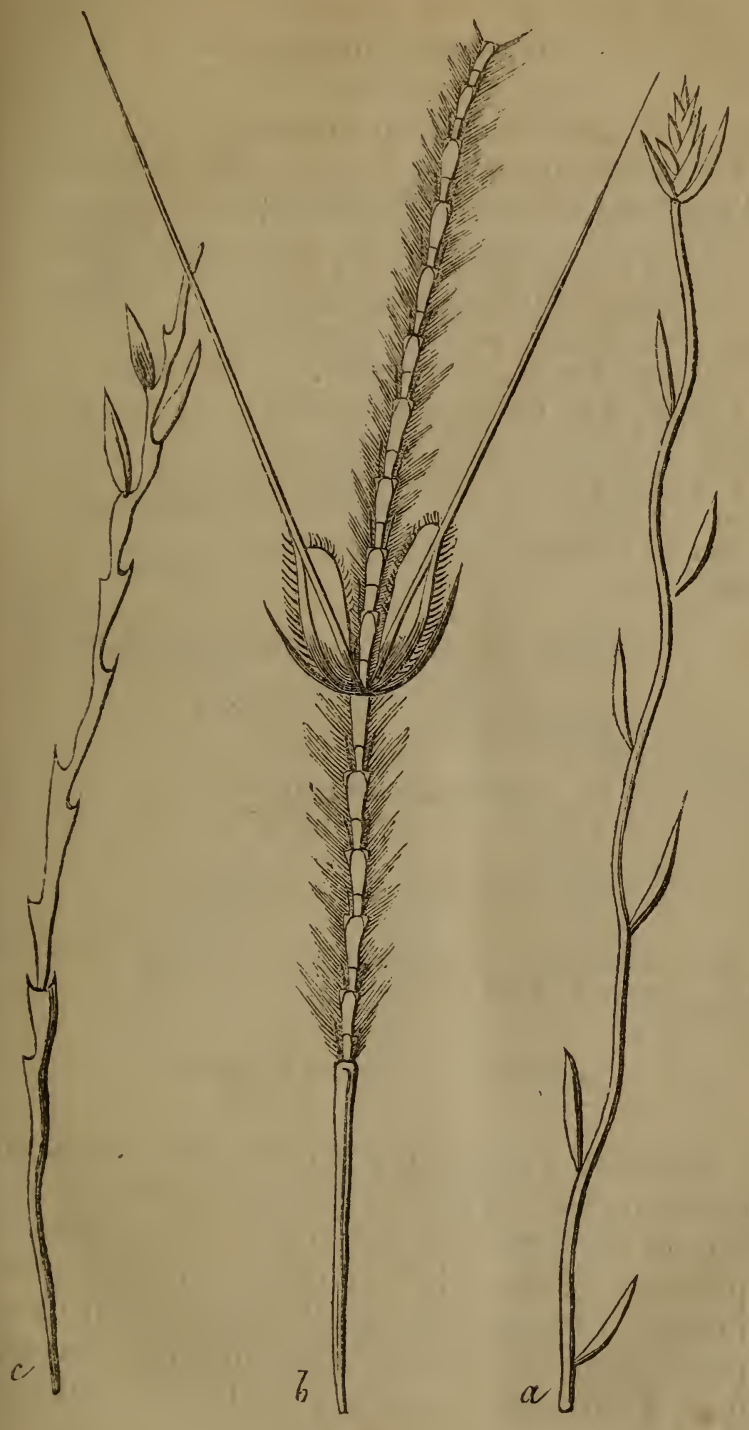

Fig. $\bar{J}(1)$.

1) a Rirhis en zigzzag. 6 hachis arlicule ch denti. c Rathis denté ferpone milatéral. 
ANALYSE DES SOUS-TRIBUS.

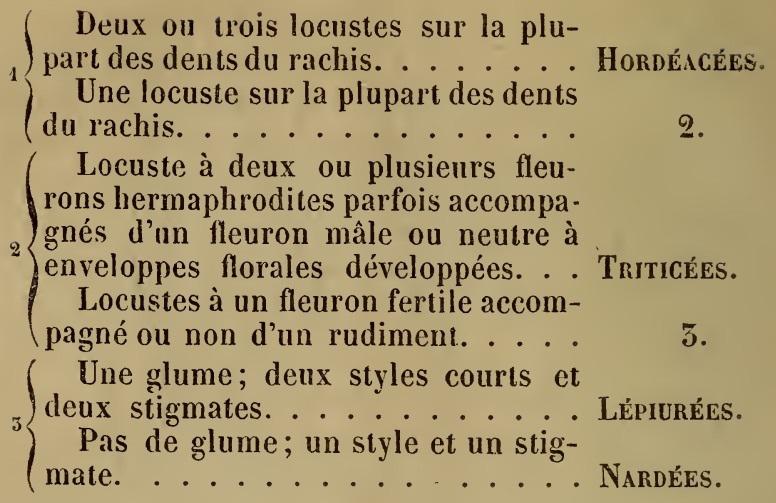

ORDRE DE SUCCESSION DES SOUS-TRIBUS.

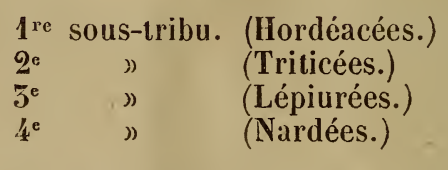

re sous-tribu. - Hordéacées.

Les hordéacées présentent deux ou trois locustes sur chaque dent du rachis.

$$
\text { Genre 2. - elyme (elymus L.). }
$$

Locustes à deux ou plusieurs fleurons fertiles, rarement un seul par avortement, réunies au nombre de deux ou trois sur chaque dent du rachis; paillettes placées plus ou moins en dehors de la locuste, l'ensemble des paillettes simulant une espèce d'involucre; paléole extérieure aristée ou mutique, l'intérieure bicarénée, entière, émarginée; 
paléolules 2 , entières ou bilobées, à lobes inégaux, glabres, poilues ou ciliées ; ovaire plus ou moins renflé ou turbiné; stigmates 2 , subsessiles, divariqués ou réfléchis, plumeux; grain oblong, un peu convexe, ventru sur l'une des faces, comprimé ou presque plan sur l'autre, parcourue par un sillon libre ou adhérant aux paléoles. Epi simple.

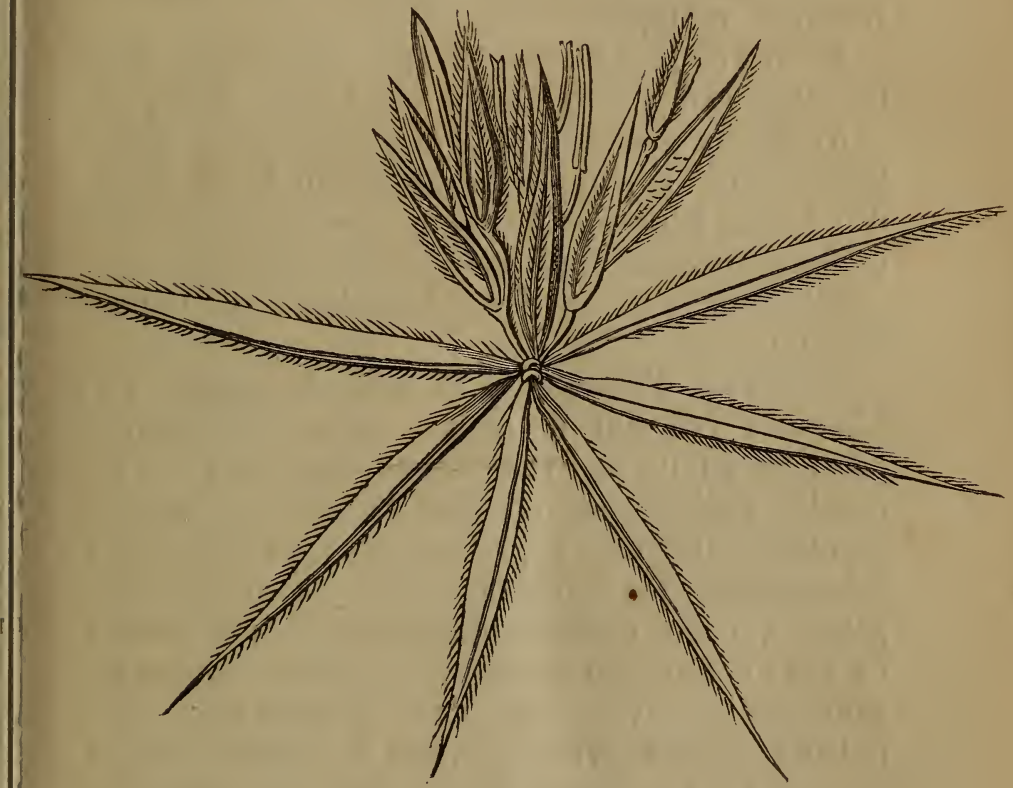

Fig. 4.

Locuste d'èlyme.

ANALYSE DES ESPÈCES.
$\left\{\begin{array}{c}\text { Paléole mutique externe munie d'une arête à } \\ \text { Paléole eine deux ou trois fois plus longue }\end{array}\right.$
2.
qque le fleuron. . . . . . . . . . E. D’Eunope $(c)$. 
of Locustes ternées. . . . . . E. Des Sables (a). Locustes géminées. . . . . . E. Géniculé (b).

a) Elyme des sables. (Elymus arenarius L.) Souche longuement traçante; chaumes s'élèvant de un à deux mètres de hauteur, garnis de feuilles larges et longues, roides, scabres, planes ou un peu enroulées, très-glauques, pointues; épi long, droit, blanchâtre, velu, à locustes ternées bi ou triflores, mutiques.

Fleurit de juin à août; vivace.-Se trouve dans les sables arides de la Campine et dans les dunes (1).

b) Elyme géniculé. (Elymus geniculatus Nob.) Cette plante se rapproche beaucoup de la précédente dont elle ne se distingue que par son épi qui est un peu arqué et par ses locustes géminées.

Elle se trouve dans les mêmes lieux que la précédente.

c) Elyme d'Europe. (Elymus Europous L.) Chaumes s'élevant de trois à quinze décimètres; nu, lisse ou un peu rude vers le haut, un peu genouillé inférieurement; feuilles planes, larges, pointues, glabres ou pubescentes; les inférieures très-longues; les supérieures plus courtes que les gaînes qui sont fortement hérissées, à poils dirigés en bas; épi un peu comprimé; locustes ternées ou géminées par avortement de la locuste moyenne; paléole externe aristée; arêtes à peine deux ou trois fois plus longues que les fleurons, celles des locustes latérales plus longues.

(1) Nous aurions pu nous dispenser l'indiquer les lieux où l'on trouve les espèces, attendu que la troisième partie s'oceupe de cet objet; mais nous avons cru qu’il était nécessaire d'indiquer sommairement après chaque description les endroits prineipaux où on les reneontre, sauf à revenir avec plus de détail sur les meilleures d'entre elles, dans la troisième partie. Ces indications sont le complément indiqqensable de toute deseription botanique. 
DEUXIÈye PARTIE. - ANALYSES ET DESCRIPTIONS. 75

Fleurit de juin à juillet; croì dans les sols argileux, schisteux, humides et froids, sur les bords des bois et aux lieux ombragés des provinces de Namur, Liége, Hainaut el Luxembourg.

$$
\text { GENRE 3. - ORGE (IIORDEUM L.). }
$$

Ce genrea beaucoup de rapport arec le précédent,

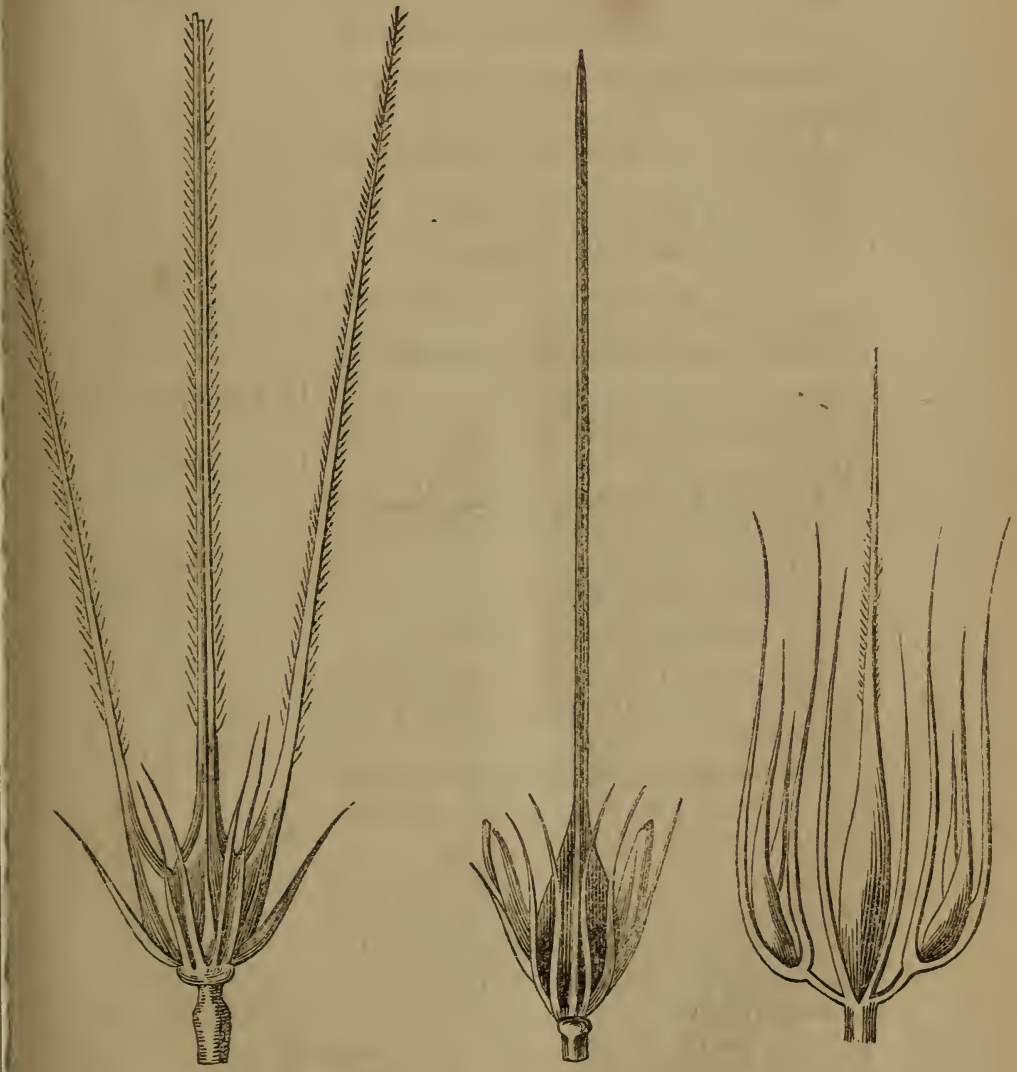

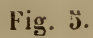

e de locustes fertiles de l'orge vulgaire. Id. de l'orge distique. Id. de l'orge des pres. 
dont il ne se distingue que par ses locustes à un fleuron accompagné d'un rudiment pédicelliforme barbu ou glabre et par la constitution de ces mèmes locustes qui sontquelquefois toutes fertiles, ou dont la moyenne de chaque groupe l'est seulement, les latérales étant mâles ou neutres.

La figure $\breve{~ d o n n e ~ l e s ~ t r o i s ~ v a r i a t i o n s ~ p r i n-~}$ cipales.

\section{ANALYSE DES ESPÈCES.}

Locustes latérales mâles, neutres ou stériles.

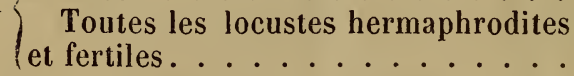

2 Locustes latérales aristées. . . . .

$\int$ Toutes les paillettes des locustes $5\left\{\begin{array}{c}\text { non ciliées. } \\ \text { Paillettes de la locuste médiane ci- }\end{array}\right.$ liées ................

2.

7.

5.

5.

4.

0. DES SoURis (b).

0. DES PRÉS (a). vivace................

Paillettes intérieures des locustes latérales semilancéolées; plante annuelle. . . . . . . . . . 0. Maritime (c). s) Souche vivace, bulbeuse. . . . . 0. Bulbeuse (d)

Plante annuelle non bulbeuse. . $\quad 6$.

(Epi comprimé, à peu près d'égale largeur dans toute son étendue; arêtes apprimées.

6 Epi comprimé pyramidal, c'est-à-dire plus large à la base qu'au sommet; arêtes écartées et divariquées. . . . . 0. pyramidale (f).

Épi plus ou moins courbé; locustes disposées sur six rangs, dont deux rangs latéraux inégalement distants,

7 plus rapprochés, et deux opposés, plus proéminents. ...............

Epi droit, court, épais, à six rangs également distants les uns des autres. 0. a six rangs (g). 
DEUXIEME PARTIE. - ANALYSES ET DESGRIPTIONS. $7 \%$

A. - locustes latérales hlales, neutres ou

STÉrILES.

$\S$ I. Locustes latérales aristées.

a) Orge des prés. (Hordeum, pratense Huds.) Chaumes nombreux, de cinq à dix décimètres, grêles, dressés, parfois renflés en bulbes à la bașe ; feuilles étroites, planes, les gaines des inférieures plus ou moins pubescentes; épi un peu cylindrique ou comprimé; paillettes non ciliées, scabres; locustes latérales à fleuron stérile rudimentaire, assez courtement aristées. Vivace, fleurit en juinjuillet.

Cette espèce offre deux variétés qu'on a prises pour des espèces distincles : ce sont l'orge des prés vulgaire, qui a un épi un peu cylindrique, presque à six angles, d'un vert foncé ou purpurin, et l'orge seigline qui a un épi comprimé, distique, verdâtre ou d'un jaune verdâtre. Ces deux plantes sont assez communes dans les prairies grasses et dans les prairies humeuses; elles abondent dans les riches pàturages de Dixmude et dans les prairies d'Anderlecht, de Denderbelle et des Polders. On les trouve aussi communément dans toutes les prairies qui se trouvent sur les bords de la Dendre.

b) Orge des souris. (Hordeum murinum L.) Souche annuelle formant des touffes épaisses d'où s'élèvent des chaumes de $\mathbf{3}$ à 8 décimètres, genouillés à la base et souvent feuillés jusque vers le haut; les feuilles sont abondantes, étroites, planes, molles, velues et les gaînes comprimées : la gaîne supérieure est plus ou moins renflée. L'épi est d'un jaune verdâtre, oblong, serré; les locustes latérales. 
sont assez longuement aristées et les paillettes de la locustefertile lancéolées, linéaires, ciliées. Annuelle; fleurit de juin à août.

Cette plante est commune le long des chemins et des routes, où elle pullule quelquefois, malgré les efforts de l'homme; mais elle acquiert particulièrement un grand développement le long de la lisière des murailles.

c) Orge maritime. (Hordeum maritimum Vahl.) Chaumes genouillés abondants, à demi couchés; feuilles recouvertes d'une légère couche de poussière glauque, assez courtes, larges, pointues et pubescentes; épi court, subcylindrique ou à quatre angles obtus, souvent engainé; paillettes intérieures des locustes latérales semi-lancéolées, les autres sétacées; arêtes divergentes qui impriment à l'épi, lors de sa maturité, un port très-intéressant. Annuelle; fleurit de juin à juillet. - Croît dans les endroits sablonneux à proximité de la mer et aux lieux herbeux des dunes.

$\S$ II. Locustes latérales mutiques.

d) Orge bulbeuse. (Hordeum bulbosum L.) Souche bulbeuse formant des touffes serrées de feuilles et émettant un grand nombre de chaumes feuillés de 1 à 2 mètres; feuilles larges, molles, planes; épi allongé; fleurons des locustes latérales mâles mutiques, plus courts que les glumes. Vivace; fleurit en juin.

Cette plante qui est cultivée depuis quelques années à titre d'essai n'est pas sans mérite et pourra devenir d'une grande utilité dans certaines localités de notre pays. Elle joint à une grande précocité des 
produits assez abondants et de bonne qualité comme fourragère.

e) Orge distique. (Hordeum distichum L.)Chaumes droits, lisses, d'un mètre ou plus; feuilles planes, un peu roides; épi comprimé, penché, à peu près d'égale largeur dans toute son étendue; locustes latérales mutiques, celles du milieu hermaphrodites, disposées sur deux rangs et munies d'arêtes longues, apprimées et imbriquées. Annuelle; fleurit en juin.

On cultive plusieurs variétés d'orge distique, parmi lesquelles on distingue :

L'orge à deux rangs commune. Paille d'un mètre ; balles assez épaisses, jaunâtres, adhérentes; rudiment velu-cilié.

L'orge chevalier. Paille plus élevée que dans l'espèce ordinaire; feuilles plus larges; balle plus mince, jaunâtre, pâle, y adhérente; rudiment pubescent.

L'orge d'Italie. Épi moins allongé que dans l'espèce ordinaire, plus régulier, dressé, plus élargi; et à grains plus rapprochés; paille haute et forte; grains cortiqués.

L'orge nue à deux rangs. Le grain est gros, lourd, et se détache des paléoles lors du battage.

L'orge café. Grains nus, lourds; paille cassante et très-fragile.

f) Orge pyramidale. (Hordeum zeocritum L.) Chaume droit, glabre, lisse, strié; feuilles larges, glabres, planes, rudes au toucher, fort longues et pointues; épi court, roide, comprimé, pyramidal ; arêtes longues, écartées et divariquées. Annuelle; cultivée, fleurit en juin.

B. - toutes les locustes fertiles aristées.

g) Orge it six rangs. (Hordeum hexastichum L.) Chaume droit, glabre, cannelé, lisse, 
quelquefois entièrement glauque; feuilles larges, planes, rudes au toucher et pointues : épi ovale, court, épais, droit, à six rangs également proéminents; arêtes dix à trente fois plus longues que la paléole; grain adhérant à la glumelle. Annuelle; fleurit en été.

h) Orge commune. (Hordeum vulgare L.) Chaume dressé, glabre, lisse, strié ; feuilles larges, planes, glabres, rudes au toucher, pointues. Epi oblong, plus ou moins courbé; locustes disposées sur six rangs, dont deux rangs latéraux, inégalement distants, plus rapprochés, et deux opposés plus proéminents. Arête dix à vingt fois plus longue que la paléole. Annuelle; cultivée; fleurit en juin.

On cultive diverses variétés d’orge qui sont :

L’orge carrée d'hiver. Epi allongé, penché; rudiment velu-cilié.

L'orge carrée de printemps. Épi ramassé, un peu plus court que celui d'hiver et beaucoup moins productif; rudiment à peine pubescent-velu.

L'orge carrée nue, ou orge céleste, orge nue à six rangs, petite orge nue, dont le grain, ainsi que le nom l'indique, se dégage de sa balle par le battage; le grain est brun-roussàtre, aplati et allongé.

L'orge de Guimalaye ou de Nampto, dont le grain ést nu, court, un peu arrondi et d'un brun verdâtre.

L’orge noire ou grise. Grain adhérant à la glumelle; paléoles d'un gris noirâtre.

\section{Ine sous-tribu. - Triticées.}

Locustes solitaires sur chaque dent du pachis, bi-multiflores; deux stigmales. 
DEUXIÈUE PARTIE. - ANALYSES ET DESCRIPTIONS. 79

GENRE 4. - SEIGLE. (SECALE L.)

Locustes bi-triflores; fleuron supérieur souvent stérile et assez longuement pédicellé, solitaires sur les dents du rachis auquel elles présentent l'une de leurs faces latérales; paillettes presque opposées et herbacées, linéaires, subulées, uninerviées; paléole
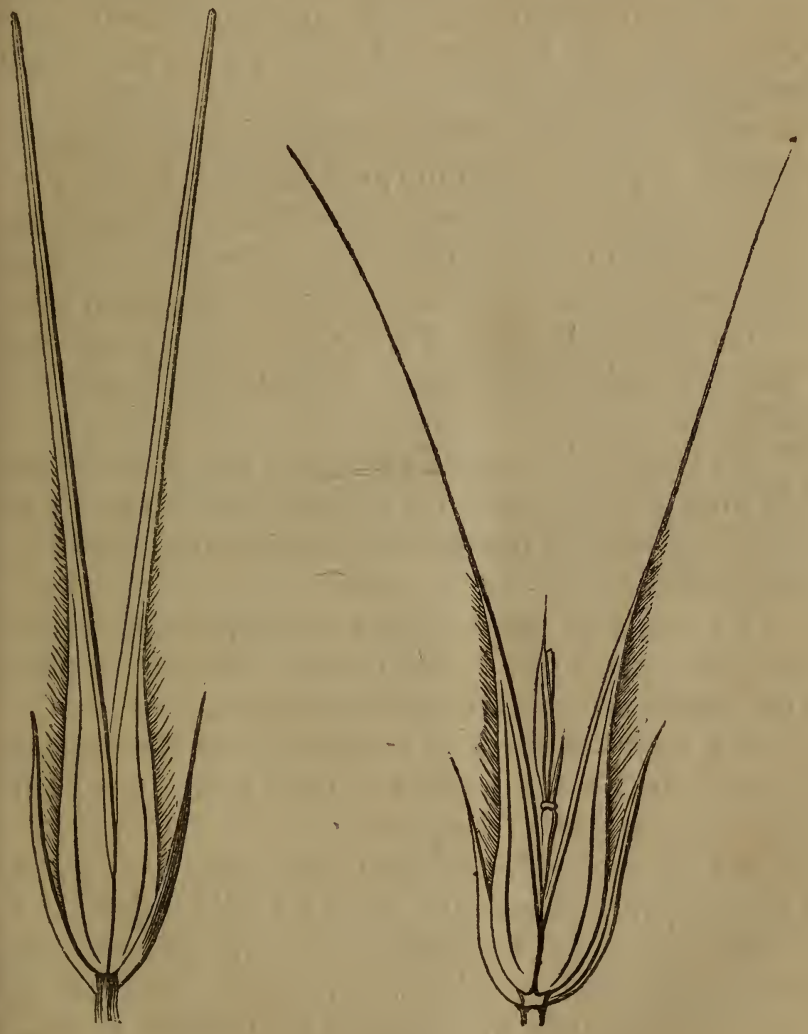

Fig. 6.

Locuste de seigle cultive biflore.

Id. triflore. 
externe roide, comprimée, carénée, à carène ciliée, se terminant en une longue arête; paléole intérieure, lancéolée, plus courte, bicarénée; paléolules 2, entières, ciliées; ovaire turbiné, poilu au sommet; stigmates 2 , plumeux, sessiles, terminaux; grain oblong, subcylindrique, aminci aux deux extrémités, présentant un sillon sur l'une de ses faces, libre entre les paléoles ; épi simple, plus ou moins comprimé, rachis pourvu latéralement d'une série de bouquets de poils, plus ou moins longs et appliqués.

Seigle cultivé. (Secale cercale L.) Chaumes et feuilles d'un vert glauque; épi allongé; locustes ordinairement à deux fleurons fertiles; paillettes plus courtes que la locuste; paléole externe se terminant en une longue arête, scabre. Annuel; fleurit en juin.

On cultive le seigle d'hiver, le seigle de mars, le seigle multicaule, le seigle de Russie et le seigle de Rome.

Le seigle d'hiver est celui qui est cultivé dans la plus grande partie de la Belgique; ce seigle ne donne guère de nombreux chaumes que dans les sols fertiles.

Le seigle de mars donne une paille plus courte et plus mince que celle du seigle d'hiver; le grain est également sensiblement plus petit.

Le seigle multicaule, seigle de la Saint-Jean, seigle du Nord, est tardif et talle beaucoup; paille et épi allongés, grain petit.

Le seigle de Russie donne un grain bien nourri et une paille abondante, mais ne talle guère.

Le seigle de Vierlande a un épi un peu plus large que celui du seigle de Russie, et un grain encore plus nourri. En rapprochant le seigle de Vierlande de notre seigle ordinaire d'hiver, cultivé dans 
DEUNIĖaE PARTIE. - ANALYSES ET DESCRIPTIONS. 81

un sol fertile, il ne peut y avoir de doute que ce seigle ne soit qu'un triage du nôtre. Il n'en est pas de mème du seigle de Rome, qui se distingue entre toutes les variétés par la vigueur de sa végétation, par sa paille forte et élevée, par son épi fortement élargi et par son grain blond qui est au moins le double plus grand que celui de notre seigle ordinaire.

\section{GENRE 5. - FRONENT. (TRITICUM L.)}

Locustes de deux àquinze fleurons, solitaires sur les dents du rachis, auquel elles présentent une de leurs faces latérales; glume à deux paillettes non subulées, presque opposées, herbacées, mutiques, mucronées ou uniaristées, aiguës ou tronquées; paléole extérieure convexe ou carénée, mutique, tronquée, mucronée, ou se terminant en une arête plus ou moins longue; l'interne bicarénée, à carène ciliée, embrassée en partie par l'extérieure; paléolules 2 ; ovaire turbiné ; stigmates 2 , terminaux, réfléchis, plumeux; grain ovoïde ou oblong, obtus aux extrémités, glabre ou velu à son sommet et sillonné; épi simple, comprimé ou'tétragrone, rarement rameux. 


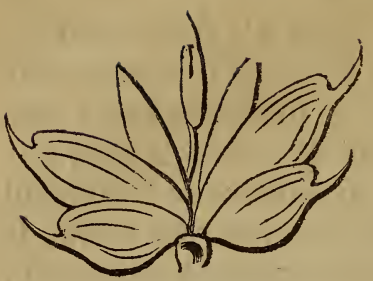

Locuste de froment cultivé.

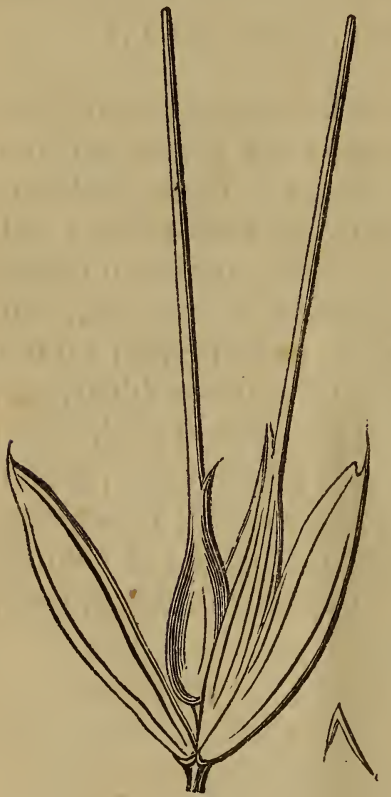

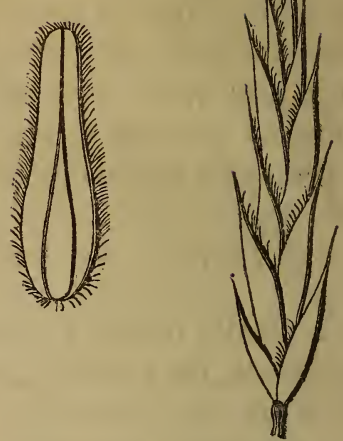

Paléole interne. Locuste de froment pinné.
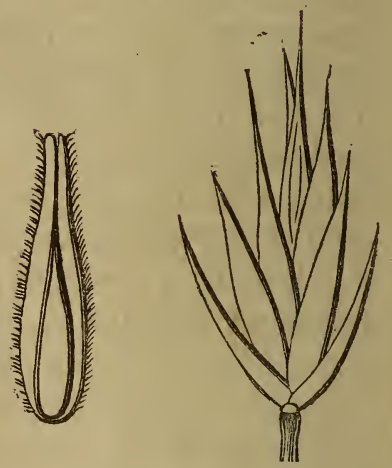

Fig. 7.

Locuste de froment de Pologne. Paléole interne.

Locuste de froment trạant (chiendent).

Glume carénée enveloppant la lo1 custe. . . . . . . . . . . F. DE POLOGNE (c). Glume n'enveloppant pas la locuste. 2.

( Paillettes ovales ou oblongues, plus ou moins ventrues ou carénées. . . . Pailletles plus ou moins convexes au dos, ni ventrues, ni carénées. . . 
DEUXIĖUE PARTIE. - ANALYSES ET DESCRIPTIONS. $8 \overline{ }$

$=\left\{\begin{array}{c}\text { Grain libre entre les paléoles; ra- } \\ \text { chis non fragile. . . . . . . . . } \\ \text { Grain étroitement renfermé entre }\end{array}\right.$

4.

5 .

( Paléole convexe, ventrue, à peine carénée dans une partie desa longueur;

4 chaume fistuleux. - dans tonte sa

F. cultivé (a). longueur une carène tranchante ; chaume plein supérieurement. . . F. TURGIde (b).

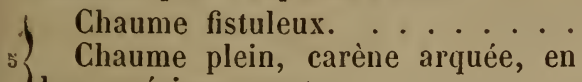
bec supérieurement. . . . . . . F. amylacé (e).

Paillettes tronquées, mucronées;épi lâchement imbriqué, incliné à la maturité; grain opaque. . . . . . F. ÉPEAutre (d).

Paillettes bidentées au sommet; carène droite supérieurement; épi étroitement imbriçué, droit à la maturité; grain demi-transparent, dur. .... F. ENGRains (f).

Paillettes très-entières, inégales entre elles; paléole interne obtuse ou 7 tronquée au sommet. ......

${ }^{7}$ Paillettes entières, égales ou presque égales entre elles; paléole exterue plus ou moins bifide ou émarginée.

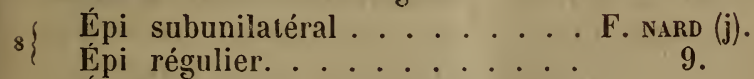

Épi composé de une à cinq locustes,

roide, dressé. Plante annuelle. . F. cıLı́ (r).

$9\left\{\begin{array}{c}\text { Épi plus ou moins incliué, de huit à } \\ \text { a }\end{array}\right.$ (treize locustes. Plante vivace. . . . .

( Souche cespiteuse n'émettant pas des rhizomes traçants; arête aussi longue ou plus longue que la paléole. F. Des Bors. (h).

10 Souche émettant des rhizomes traçants; arête sensiblement plus courte que la paléole. . ........... F. PInNé (i).

Souche cespiteuse ou presque tra-

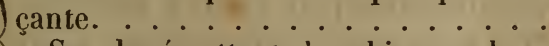
12.

${ }_{11}$ Souche émettant des rhizomes longuement traçants. 
Feuilles scabres sur les deux faces;

arête plus longue que la paléole. . . . F. dEs CHIENs (I).

12 Feuilles scabres sur l'une de leurs

faces; paléole mutique ou munie d'une

arête plus courte qu'elle. . . . . F. Rolde (k).

${ }_{13} \int$ Rachis fragile; paléoles mutiques ob-

tuses. ................... Jonatrorme. (0).

Rachis non fragile....... F. TRaÇANT (m).

A. - Paillettes ovales ou oblongues, plus ou moins Ventrues OU CarÉnÉES; SCOBINE COURTE, ÉPAISSE A LA BASE.

$\S$ I. Grain libre entre les paléoles; rachis non fragile.

a) Froment cultivé. (Triticum sativum Lam; Triticum astivum et hybernum L.) Chaumes plus ou moins nombreux, fistuleux, dressés ; feuilles larges; épi tétragone, droit; locustes à trois ou quatre fleurons ; paillettes mucronées ou aristées, comprimées au sommet, convexes-ventrues au dos, ou à peine carénées supérieurement; grain ovoïde, obtus, farineux, opaque. On en cultive deux races : le froment d'hiver et le froment d'été.Le froment d'hiver est ordinairement mutique ou submutique, tandis que le froment d'été est ordinairement aristé. Le nombre des variétés est très-considérable, mais on n'en cultive en Belgique que cinq ou six, quoiqu'il y en ait encore d'autres qui méritent d'être essayées.

1 E Épi mutique ou submutique.

\section{A. - ÉPI BLANC GLABRE.}

Blé blanc de Flandre, blé de Bergues, blé blanzé ou blazé. Épi fort, un peu aminci aux deux extrémilés; locustes très-serrées, blanches; grain hlane, oblong et tendre. 
DEUXIĖME PARTIE. - ANALYSES ET DESCRIPTIONS. 83

Blé de Hongrie. Épi ramassé; locustes serrées, blanches; grain blanc, très-raccourci (d'hiver). Ce blé a été aussi repandu sous les noms de blé anglais, blé chevalier.

Blé de Talavera. Épi lâche, long; locustes blanches; grain blanc, oblong et tendre.

Blé du Cap. Epi serré, locustes blanches, grain gros (de mars).

Blé de Saumur. Épi fort, blanc sur ses deux faces larges; pourtour des glumes d'une teinte rousse ; grain jaune ou rougeâtre, tendre (d'hiver, mais un peu plus délicat que notre froment ordinaire.

Blé hickling. Epi blanc, très-gros, compacte, élargi ; grain jaune plus raccourci, paille assez élevée, grosse et ferme. Tardif.

\section{B. - ÉPI BLANG velouté.}

Blé de tunstall (blé de haie). Épi carré, gros et grand, régulier, couvert d'un duvet blanc velouté; grain court, blanc jaunâtre. Ce froment qui est cultivé depuis quelques années dans la Flandre occidentale, y est connu sous le nom de blé d'A ustralie.

C. - Ĺpi rouge ou Jaune-roussatre.

Blé des Pays-Bas. Épi jaunâtre, pyramidal, grain roussâtre ou rouge et long.

Blé de Brabant. Épi à locustes serrées, assez large, d'un rouge roussâtre; grain fort, plein. Le mary gold red n'en est qu'une sous-variété à épi rouge brunâtre, qui se dépouille, dit-on, de tous ses caractères au bout de quelques années de culture ordinaire. Cependant, par un triage soigné, nous sommes parvenu à le conserver jusquà présent avec toutes ses qualités, et il y a plus de huit ans que nous en avons acquis la semence. 
Blé de Marianopolis. Épi long, étroit, rougeâtre; grains rougeâtres peu gros, pleins (d'hiver et de mars).

Blé du Caucase. Épi d'un rouge obscur, long, à locustes écartées; grain allongé, rougeâtre, assez dur et pesant.

Blé de mars rouge sans barbes. Épi long, à locustes supérieures courtement aristées.

Blé de mars carré de Sicile. Épi rouge brun, carré, très-court; grain rouge presque dur.

Blé d'Odessa. Epi un peu irrégulier, rougeâtre ou cuivré; locustes inégales; grain étroit, délicat (de mars).

Blé velu de Crète. Épi ròux, très-velu; grain jaune clair (de mars).

20 Épi aristé.

Blé roux de Belgique. Épi assez serré, roux; grain court, renflé, jaune d'or (d'hiver).

Blé commun barbu, blé grison. Epi lâche; locustes barbues, glabres (de mars); grain clair.

Blé du Caucase barbu. Grain allongé, rougeâtre, presque dur et pesant (de mars).

Blé du Caucase amélioré. Épi large, serré; grain blanc, court, plein (de mars).

Richelle barbue. A peu près analogue au blé du Caucase barbu, mais le grain est allongé et clair (de mars).

Blé du Cap. Épi très-allongé ; grain long, d'un blanc jaunâtre et très-pesant (de mars).

Blé de mars barbu de Toscane. Épi court, faible; paille fine. Cultivé pour sa paille (de mars).

Blé de Victoria. Épi jaune; grain rougeâtre, presque dur; paille courte, ferme (de mars).

Bléhérisson. Épi compacte, fort garni d'arêtes di- 
DEUXIENE PARTIE. - ANALYSES ET DESCRIPTIONS. 8ä

Blé de Talavera. Épi un peu plus lâche que le précédent.

Blé des Pays-Bas. Épi pyramidal, blanchâtre, quelquefois un peu jaunâtre; grain jaunâtre, roussâtre ou rouge, un peu allongé. Cette variété comprend un grand nombre de sous-variétés locales.

Blé de Saumur. A beaucoup de rapport avec le précédent, mais l'épi est plus fort, blanc sur ses deux faces larges et à pourtour des glumes d'une teinte rousse; grain jaune ou rougeàtre; d'hiver, mais un peu plus délicat que lui. Productif.

Blé de lìle Noë. Épi long, un peu làche, blanc, presque mat; grain ovoïde, blanc-jaunâtre sur la face convexe, fauve sur les côtés; bien nourri. Cette variété qui est une des plus précoces est très-glauque dans toutes ses parties. (D'automne.)

Le blé Victoria a beaucoup d'analogie avec le précédent, mais son grain est plus jaune : il n'estpas non plus glauque comme celui-ci. (D'automne.)

Blé Hichling. Épi presque en massue allongée, blanc, très-gros, compacte; grain obovoïde, jaune; paille assez élevée, grosse et ferme. Tardif. La sousvariété connue sous le nom de blé schouppe, blé bel épi, blé de Dumesnil St-Firmin est plus vigoureuse.

Blé de Hongrie. Épi court, blanchàtre; locustes ramassées ; grain blanc, raccourci. (D'hiver.)

Talavera de Bellevue. Épi blanc, làche; grain blanc, moyen, beau. (De mars.)

B. - Épl blanchatre velouté.

Blé de Tunstall (blé de haie). Épi gros et grand, carré, régulier, couvert d'un duvet blanc velouté; grain blanc-jaunàtre. Il est cultivé depuis quelques années dans la Flandre occidentale, el y est comnu sous le nom de blé d'A ustralie. Très-précoce. 


\section{C. - épi roux, Rouge ou rovgeatre.}

Blé de Brabant. Épi à locustes serrées, carré, assez large, d'un rouge-roussâtre; grain roux, fort plein; péricarpe et épisperme (pellicule) minces.

Le mary gold red a beaucoup de rapport avec le précédent, mais ses locustes sont un peu moins serrées, d'un rouge plus foncé; son grain est moins riche en fleur fine. Le rouge d'Ecosse en est à peine une sous-variété. Le grain est rougeâtre.

Blé red chaff Dantzick. Épi long, à locustes assez serrées, rouges; grain jaune. Très-productif.

Blé de mars rouge sans barbes. Épi long, à locustes supérieures courtement aristées.

Blé de Marianopolis. Épi fluet, rougeâtre, ainsi que le grain. (D'hiver et de mars.)

Blé de mars carré de Sicile. Épi rouge-brun, carré, très-court; grain rouge ; presque dur.

\section{2。 Épi aristé.}

Blé roux de Belgique. Épi assez serré, roux; grain jaune ou rougeâtre (d'hiver).

Bléblanc de Belgigue. Épi assez serré, blanc-jaunâtre; grain bien nourri, jaune ou roux (d'hiver).

Blé commun barbu, blé grison. Épi lâche; locustes barbues, glabres, grain clair (de mars).

Blé du Caucase. Grain un peu allongé, jaunâtre ou rougeâtre, presque dur et pesant (de mars et d'automne). Sous-variété : Caucase amélioré.

$B l e ́$ du Cap. Épi très-allongé; grain long, d'un blanc-jaunâtre et très-pesant (de mars).

Blé de Victoria. Epi jaune; grain rougeâtre, presque dur; paille courte, ferme (de mars).

Bléhérisson. Épi compacte, fort, garni d'arêtes di- 
DEUXIÈME PARTIE. - ANALYSES ET DESCRIPTIONS. 89)

\$1. Grain étroitement enveloppé par la glumelle; rachis fragile.

d) Froment épeautre. (Triticum spelta L.) Plante très-tallante; chaumes fistuleux; épi presque tétragone ou comprimé parallèlement, lâchement imbriqué, incliné à la maturité; locustes la plupart à trois ou quatre fleurons; paillelles ovales, larges, tronquées, mucronées, à carène presque droile supérieurement; grain triquètre, allongé, pointu et opaque. Annuel.

On cultive deux races de cette espèce : l'une est à grains rouges etl'autre à grains blonds. Ces deux races ont produit quelques variétés dont les caractères se tirent de la présence ou de l'absence d'arêtes; on y signale comme bons l'épeautre rouge sans barbes et l'épeautre rouge barbue.

e) Froment amidonnier. (Triticum amyleum Ser.) Chaumes peu nombreux, pleins, glauques; feuilles larges, glauques; épi comprimé transversalement; locustes étroitement imbriquées, à trois ou quatre fleurons; paillettes terminées insensiblement par un large mucron; carène comprimée, très-saillante et arquée au sommet, ayant ses côtés convexes. Grain triquètre, allongé, pointu et renflé. Annuel.

On en cultive deux variétés, le froment amidonnier à épi blanc et le blé amidonnier à épi roux.

f) Froment engrain. (Triticum monococum L.) Chaumes assez nombreux, fistuleux, fins et roides; feuilles peu larges, vertes, un peu ciliées: ligule courte, tronquée, denticulée; épi comprimé transversalement, étroitement imbriqué, droit ; locustes à deux ou trois fleurons glabres, dont un ou deux fertiles et aristés; paillettes fortement ca- 
rénées et irrégulièrement bidentées au sommet, dents plus ou moins aiguës et vertes; paillettes à un côté convexe ; carène droite au sommet, non incourbée; grain imparfaitement triquètre, comprimé, demi-transparent ou presque opaque. Dans les expositions chaudes et élevées, le grain acquiertbeaucoup d'analogie avec le riz. On en cultive deux variétés : dans l'une, la plupart des locustes ne contiennent qu'un seul fleuron fertile, rarement deux; l'autre a des locustes la plupart à deux fleurons fertiles.

B. - Palllettes pies ou molns arrondies aU dos, Ni ventRUES, NI CARÉNÉES; SCOBINE FILIFORME.

1. Paillettes entières très-inégales entre elles; paléole externe obtuse ou tronquée au sommet.

g) Froment des bois. (Triticum sylvativum Moench.) Souche cespiteuse produisant des touffes serrées, formées de faisceaux de feuilles et de tiges nombreuses de quatre à douze décimètres, garnies de feuilles lancéolées-linéaires, planes, molles, velues, ainsi que les gaines et les nouds; locustes au nombre de huità douze, minces, linéaires, droites, d'un vert un peu grisâtre, contenant dix à quinze fleurons; paléole externe à cinq nervures; arêtes droites un peu ondulées, celles des fleurons supérieurs plus longues que la paléole; paillettes lancéolées, aiguës, striées.

Cette plante vivace fleurit tout l'été et a fourni deux variétés : l'une à locustes glabres et l'autre à locustes pubescentes.

h) Froment pinné.(Triticum pinnatum Moench.) Souche émettant des rhizomes longuement traçants; chaumes dequatreà neuf décimètres, roides; 
feuilles linéaires ou lancéolées-linéaires, planes, d'un rert un peu glauque, pointues; gaines glabres ou presque glabres; épi droit ou un peu penché; huit à douze locustes contenant huit à dix-sept fleurons; paillettes striées; paléoles externes rudes ou lisses, gglabres ou velues, striées au sommet; arète plus courte que la paléole, ou nulle.

On trouve en Belgique diverses variétés accidentelles; ainsi il y en a à locustes velues et à locustes glabres, lisses, droites ou arquées, à épi ramassé et à feuilles très-roides, à locustes inférieures géminées et à locustes mutiques.

Toutes sont vivaces et fleurissent de juin à août; on les trouve assez abondamment dans les lieux arides et les bois.

i) Froment cilié. (Triticum ciliatum D. C.) Plante annuelle. Chaumes nombreux et rameux, de trois à cinq décimètres, géniculés à la base ; feuilles planes, ciliées; épi de trois à sept locustes comprimées, de six à trente-deux fleurons; arête souvent plus longue que la paléole externe. Fleurit en juin; croît dans les endroits secs, dans les sables des dunes près de Nieuport, où elle se montrequelquefois en grande abondance.

j) Froment nard. (Triticum nardus D. C.). Plante annuelle. Chaumes nombreux ou solitaires, de quatre à vingt-cinq centimètres, grèles; feuilles étroites, sétacées, canaliculées ou enroulées; épi subunilatéral, linéaire; locustes sessiles ou subsessiles verdàtres, assez petites, ovales-oblongues, de trois à sept fleurons; paléole externe lancéolée-linéaire, souvent longuement aristée, rarement mutique.

On en trouve en Belgique deux variétés : l'une est glabre ou presque glabre; l'autre a les feuilles, les gaînes et les locustes pubescentes. Annucl. 
Dans les terrains sablonneux riches en calcaire.

2. Paillettes entières, égales ou presque égales entre elles; paléole interne plus ou moins bifide ou émarginée.

k) Froment roide. (Triticum rigidum Schr.) Souche cespiteuse ou presque traçante; chaumes peu nombreux, de quatre à huit décimètres ou plus; feuilles linéaires-lancéolées, scabres sur l'une de leurs faces; épi distique; locustes supérieures rapprochées à quatre ou cinq fleurons, les inférieures notablement espacées; paillettes oblongues à cinq ou sept nervures, très-obtuses ou tronquées, de moitié plus courtes que la locuste; paléoles trèsobtuses, mutiques ou aristées; rachis rude. Vivace; fleurit de juin à août.

Endroits stériles et arides de la province de Liége.

l) Froment des chiens. (Triticum caninum L.) Souche cespiteuse; chaumes de quatre à dix décimètres; feuilles scabres sur les deux faces; épi distique, allongé, à locustes souvent rapprochées, de quatre à six fleurons; paillettes lancéolées à trois ou cinq nervures, acuminées; paléole externe munie d'une arête plus longue qu'elle; rachis scabre. Vivace; fleurit en juin-juillet.

Haies, lisière des bois et lieux incultes.

m) Froment jonciforme (Triticum junceum L.) Souche émettant des rhizomes longuement traçants; chaumes de cinq à dix décimètres; feuilles à rejets stériles, très-pubescentes à la face supérieure; épi distique à rachis fragile, même avant qu'il ait atteint sa complète maturité; locustes es- 
pacées à cinq ou huit fleurons, grandes; paillettes lancéolées-linéaires, à neuf ou onze nervures plus ou moins clairement marquées, d'un tiers plus courtes que la locuste; paléoles mutique, obtuse. Vivace, fleurit en juillet.

Dans les endroits sablonneux, dans les dunes.

n) Froment tracant. (Triticum repens L.) Souche émettant des rhizomes longuement traçants; chaume de cinq à quinze décimètres; feuilles scabriuscules sur une de leurs faces; épi distique; locustes de quatre à dix fleurons; paillettes lancéolées à cinq ou sept nervures, obtuses, aiguës ou acuminées; paléole externe acuminée, obtuse, mutique ou aristée; rachis souvent scabre.

Ce froment présente un nombre considérable de variétés qui ont été tour à tour considérées comme des espèces : les unes sont propres aux champs cultivés; les autres s'établissent de préférence dans les dunes et les sables maritimes où elles acquièrent une vigueur et un port qui ont suffi, aux yeux de quelques botanistes, pour en former des espèces distinctes. Nous ne nous efforcerons pas de leur découvrir des caractères, car il n'y en a pas qui aient assez d'importance pour qu'on s'y arrête sérieusement : ce sont le froment littoral (Triticum littorale H.); le froment pointu (Triticum pungens); le froment aigu (Triticum acutum); le froment à fleurs obtuses (Triticum obtusiflorum) et le froment intermédiaire (Triticum intermedium). 
GENRE 6.- OEGYLOPE. (OEGYLOPS L.)

Locustes contenant ordinairement deux fleurons fertiles, souvent accompagnés d'un fleuron supérieur stérile, sessiles et solitaires sur chaque dent du rachis; glumeà deux paillettes ovales, tronquées, striées, terminées par deux, trois, quatre ou cinq arètes subulées; paléole extérieure ovale, terminée par deux ou trois arètes; paléole intérieure lancéolée, droite, plus ou moins émarginée; ovaire plus ou moins turbiné : styles 2, réfléchis; stigmates 2 poilus. Le fleuron mâle ne diffère de l'hermaphroditeque par l'absence ou la réduction du pistil; grain oblong.

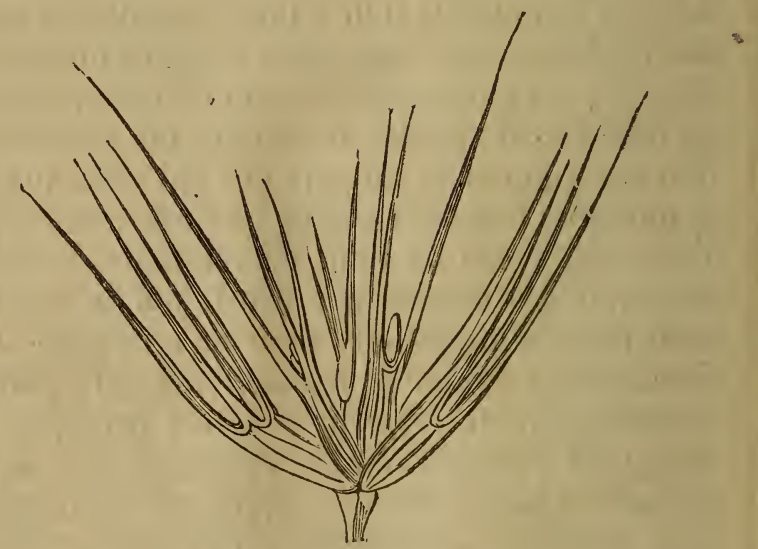

Fig. 8.

Locuste d'œgylope ovale.

Épi gros, court et ovale; paillettes

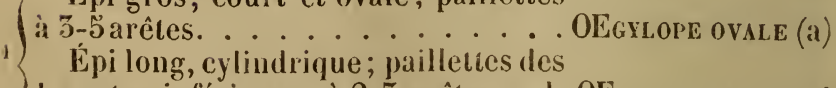

locustes inféricures à 2-3 arêtes, cel- OEgylope allonge:

les des locustes supérieures à 3 arcites.

(b). 
DECXIÈnE PARTIE. - ANALYSES ET DESCRIPTIONS. 93

a) OEgylope ovale. (OEgylops ovata L.) Chaumes rameux, de trois à cinqdécimètres, ascendants, coudés; feuilles un peu velues, ciliées; ligule courte ciliée ; épi gros, court, ovale; paillettes dures, coriaces, nerveuses, bombées et hispides, surmontées chacune de trois à cinq arêtes; paléole extérieure plus courte que les arètes qui la terminent. Annuelle; fleurit en juin et en juillet.

Se rencontre dans les terrains sablonneux et arénaires ; très-rare (?).

b) OEgylope allongée. (OEgylops triuncialis L.) Chaumes articulés, dressés, de cinq à huit décimètres; feuilles caulinaires courtes, les inférieures molles, plus larges, réunies en gazon ; épi long, cylindrique, grêle, pauciflore; paillettes des locustes inférieures munies de deux ou trois arêtes scabres, ainsi que toute la locuste; celles des locustes supérieures terminées par trois arètes très-longues, scabres hispides. Annuelle; fleurit en juin et juillet.

Dans les mèmes endroits, très-rare (?).

\section{GeNRE 7. - iVRaie (LOLiUM L.).}

Locustes de trois à trente fleurons, comprimées, solitaires sur les dents du rachis auquel elles présentent le dos des fleurons; glume des locustes terminales à deux paillettes; glume des locustes latérales presque constamment à une seule paillette persistante sur le rachis; paléole extérieure mutique, ou munie d'une arête au-dessous du sommet, ordinairement échancrée ou bifide; paléole intérieure plus courte, bicarénée, à carène plus ou moins ciliée; paléolules 2 entières ou plus ou moins divisées, lobées ou échancrées; ovaire un peu turbiné, glabre; stigmates 2 terminaux sessiles 
ou subsessiles; grain oblong, plus ou moins convexe sur l'une des faces, canaliculé ou comprimé sur l'autre, adhérant aux paléoles, ou ne s'en déta-. chant point par le battage.
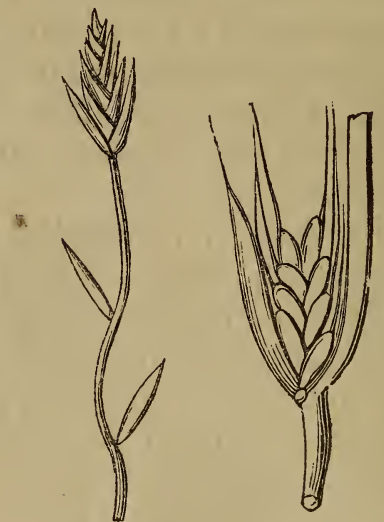

Fig. 9.

Portion d'épi de l'ivraie vivace.

Portion d'épi

de l'ivraie enivrante.

ANALYSE DES ESPÈCES.

Feuilles pliées-carénées dans leur

${ }_{1}$ jeunesse. ............. Ivraic vivace (e).

1 Feuilles enroulées dans leur jeu-

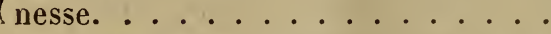

2.

$2\left\{\begin{array}{c}\text { Glume égalant ou dépassant la lo- } \\ \text { custe. . . . . . . . . . . . . . . . . } 3 .\end{array}\right.$

(Paléole interne ovale-oblongue. . Ivraie des champs

$5\{$ Plante annuelle.

Paléole externe oblongue-lancéolée.

4.

( Plante annuelle n'émettant pas ou

n'émettant gnère de fascicules le feuil-

les stériles; locustes à 15-50 fleurons. Ivrate ribfrel (c).

4 Plante vivace émettaint un grand

nombre de fascicules de feuilles stéri-

les; locustes à 5-13 flenrons. . . . Ivrane n'Italue (d). 
a) Ivraie enivrante. (Lolium temulentum $\mathbf{L}$. Lolium speciosum Bieb.) Plante annuelle ne formant pas de fascicules de feuilles stériles; chaume droit, fort, de dix à onze décimètres, lisse ou rude, surtout vers le haut; feuilles linéaires-lancéolées, glabres ou un peu hérissées, scabres; ligule courte; locustes elliptiques de trois à onze fleurons; paléole ovale, obtuse, munie d'une arète plus ou moins longue, égalant ou dépassant souvent la longueur de la paléole; paillettes fortes, roides, striées, de même iongueur ou plus longues quela locuste; grain saillant sourent latéralement à la maturité.

Var.) Itraie remarquable. (Lolium speciosum Bieb.) Arête courte, ondulée ; chaume fort et dressé.

Les Lolium decipiens et lucidum de M. Dumortierappartiennent, comme variétés, à cette espèce: la première a une arête caduque et un chaume scabre, et l'autre une arête persistante et un chaume très-lisse. Annuelle; fleurit en juin et en juillet.

Dans les moissons; surtout dans l'avoine, l'orge et le seigle.

b) Ivraie des champs. (Lolium arvense With.) Plante annuelle n'émettant pas de fascicules de feuilles stériles; chaumes peu nombreux, peu fermes et hauts de cinq à onze décimètres; feuilles enroulées, dans leur jeunesse d'un vert tendre; épi allongé; locustes assez espacées, de cinq à treize fleurons; paléole externe ovale-oblongue, très-obtuse, renflée à la maturité et munie d'une arête peu consistante et assez courte, quelquefois nulle; paillettes plus courtes que la locuste; grain saillant, sourent visible latéralement à la maturité. Annuelle; fleurit en juin et en juillet.

Se trouve dans le lin et les terres riches qui ont porté 
du lin. La présence de l'ivraie des champs dans une linière est un indice presque certain que la semence est d'origine russe, ou provient d'une culture du pays peu soignée.

- c) Ivraie rieffel. (Lolium rieffelianum Dm.) Plante annuelle n'émettant pas ou n'émettant guère des fascicules de feuilles stériles; chaume fort, de six à dix-huit décimètres, dressé ou genouillé, lisse ou un peu scabre; feuilles scabres, larges, épaisses, enroulées dans leur jeunesse; ligule courte; épi long, muni de grandes locustes de treize à trente fleurons; paléole externe ovaleallongée, un peu obtuse ou denticulée, pourvue d'une arête assez longue ou presque nulle; paillette de moitié plus courte que la locuste.

L'Ivraie Bailly n'en est qu'une variété à fleurons supérieurs courtement aristés et les inférieurs mutiques ou submutiques.

Annuelle. - Dans les champs, parmi le trèfle.

d) Ivraie d'Italie. (Lolium italicum L. Lolium boucheanum Kunth.) Plante vivace émettant un grand nombre de fascicules de feuilles stériles; chaumes assez nombreux, lisses ou scabres, de sept à quinze décimètres; feuilles assez larges, enroulées dans leur jeunesse; ligule courte; épi allongé; locustes assez rapprochées; paléole externe ovalelancéolée, munie d'une arête droite ou un peu ondulée; paillettes plus courtes que la locuste.

Fleurit en juin et en juillet.

Dans les champs de trèfle et cultivés en prairies artificielles.

e) Ivraie vivace. Raygras d'Angleterre. (Lolium perenne L.) Plante vivace èmettant un grand nombre de fascicules de feuilles stériles; chaumes droits ou un peu obliques, de quatre à huit déci- 
DEUXIÈME PARTIE. - ANALYSES ET DESCRIPTIONS. 99

mètres, scabres ou lisses; feuilles pliées dans leur jeunesse, glabres; épi allongé, à locustes aplaties contenant trois à seize fleurons, lancéulées, aiguës, mutiques ou mucronées au sommet; paillettes beaucoup plus courtes que la locuste.

Cette plante offre beaucoup de variations; nous nous bornerons à indiquer les principales.

Ivraie ténue. (Lolium tenue L.) Locuste à trois ou quatre fleurons formant un épi grêle, comprimé ; chaume droit, filiforme. glabre, de trois à quatre décimètres. On la troure dans les endroits peu fertiles, tandis queles suivantes appartiennent aux sols riches et féconds.

Ivraie en crête. (Lolium cristatum Tin.) Locustes composées, ramassées, fort courtes à sept ou neuf fleurons.

Ivraie rameuse. (Lolium ramosum Tin.) Épi rameux, décomposé.

Ivraie bipartite. (Lolium bifarium Dm.) Locustes étalées, séparées en deux parties.

Ivraie unilatérale. (Lolium secundum Tin.) Locustes ramassées, tournées vers un seul côté et simulant un épi unilatéral.

Vivace; fleurit tout l'été. - Dans les prés, les pâturages et les prairies, le long des chemins, partout enfin où l'herbe peut se développer.

III' sous-iribu. - Lépiurées.

L'épi est régulier; les locustes sont solitaires sur chaque dent du rachis qui est scrobiculeux; la glume est à deux paillettes; il y a deux stigmates sortant vers le milieu ou vers la base dufleuron fertile qui est accompagné d'un rudiment subclaviforme. 
GENRE 8. - LEPIURE (LEPIURUS DMTR.).

Locustes à un fleuron fertile accompagné d'un rudiment subclaviforme, solitaires à chaque articulation du rachis scrobiculé; glume à deux paillettes coriaces, herbacées; paléoles membraneuses mutiques; paléolules 2, entières dentées ou lobées, plus ou moins renflées à la base; deux ou trois étamines; ovaire turbiné; styles 2, courts; stigmates 2, poilus, sortant vers le milieu ou vers la partie inférieure du fleuron; grain oblong, entouré par les paléoles. Epi simple, fragile à la maturité.

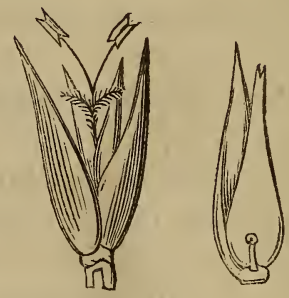

Fig. 10.

Locuste

\{Épi arqué $\ldots \ldots \ldots \ldots$ Lepiure arqué (a). \{Épi droit . . . . . . . . LePIURE ÉLANGÉ (b)

a) Lepiure arque. (Lepiurus incurvatus Dmtr.) Chaume grèle, rameux, couché à la base; feuilles planes, étroites, les radicales longues ; ligule presque nulle ; épi arqué, terminé en pointe; locustes en quelque sorte cachées dans les enfoncements

- du rachis, de manière que l'épi n'est guère plus gros que le reste du chaume. Annuel; fleurit tout l'été. 
DEUXIÈME PARTIE. - ANALYSES ET DESCRIPTIONS. 104

Croit aux bords de la mer, dans les dunes, etc.

b) Lepiure élancé. (Lepiurus strigosus Dmrt.) Cette espèce se distingue de la précédente par son chaume droit, ses feuilles caulinaires subulées, sétacées, son épi un peu plus grêle, subulé, droit, et par ses paillettes qui sont écartées après la floraison. Annuel; fleurit en été.

Se trouve dans les mêmes lieux que le précédent.

\section{IVe sous-tribu. - Nardées.}

Épi subunilatéral; locustes solitaires sur chaque dent membraneuse du rachis; glume nulle; stigmate filiforme, très-long, sortant au sommet du fleuron.

$$
\text { GENRE 9. - NARD (NARdUS L.). }
$$

Locustes solitaires à chaque dent membraneuse du rachis qui est excaré ; glume nulle; glumelle à

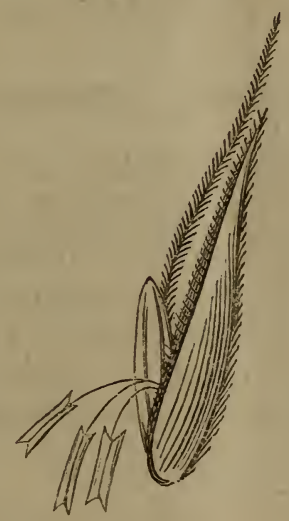

Fig. 11.

Locuste du nard elancé. 
deux paléoles, l'extérieure longue, très-aiguë, membraneuse; l'intérieure également membraneuse, linéaire-lancéolée; paléolules nulles; ovaire oblong; style unique; stigmate solitaire, terminal, filiforme. très-long, pubescent, sortant au sommet du fleuron; grain linéaire-oblong, aigu aux deux extrémités, mais plus étroit supérieurement, enveloppé par la glumelie.

Nard serré. (Nardus strictaL.) Souche courte, horizontale, émettant sur toute sa longueur des fascicules de feuilles formant une touffe dense; chaumes de un à cinq décimètres, grêles, roides, dressés, nus à cause des nœuds qui sont rapprochés à la base; feuilles sétacées, d'un vert un peu glauque, les radicales rassemblées en gazon, celles du centre droites et celles de la circonférence étalées; locustes verdâtres ou un peu violacées, subulées, formant par leur écartement un angle aigu avec le rachis, disposées en un épi unilatéral et allongé. Vivace; fleurit en juin.

Dans les lieux secs, sablonneux, montagneux et stériles.

IIIRe tribu. - Festucrées.

Les festucacées présentent une inflorescence en panicule plus ou moins rameuse, rarement simple; les locustes pédonculées contiennent deux ou plusiers fleurons fertiles, très-rarement un seul accompagné d'un ou de plusieurs fleurons neutres; paléole externe mutique ou munie d'une arête épidorsale ou terminale, jamais tordue ni géniculée. 

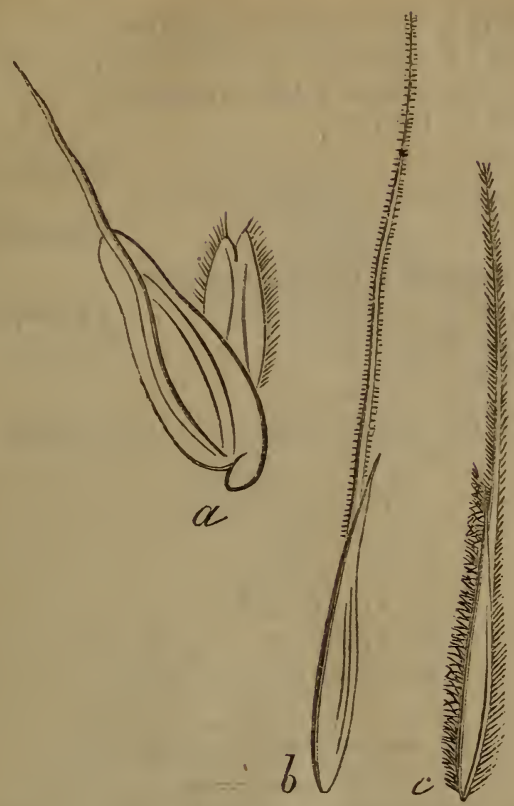

Fig. $12(1)$.

ANALYSES DES SOUS-TRIBUS.

Panicule spiciforme unilatérale, à locustes fertiles entremêlées de locustes stériles réduites à des bractées jinnées ou pectinées. . . . . . C. Crirosurées.

Pas de locustes stériles réduites à des bractées pinnées ou pectinées. . .

2.

Locustes à 2-7 fleurons; fleuron inférieur mâle glabre, etles autres munies d'un involucelle de longs poils soyeux. Arundréés.

Locustes bi ou multiflores; fleuron inférieur jamais mâle, glabre, et les autres involucellés. ........

3.

(1: a Fleuron du brome mou; $b$ paléole externe du brome stérile; c paléole externe d'une fétuqque. 
Glume large, enveloppant presque . 5 entièrement la locuste. .......

Glume n'enveloppant pas presque entièrement la locuste. ..... . 5 .

$\left\{\begin{array}{l}\text { Paléole externe carénée. . . . Sescériées. } \\ \text { Paléole externe arrondie, convexe }\end{array}\right.$

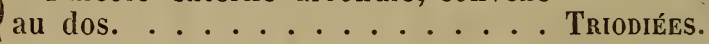

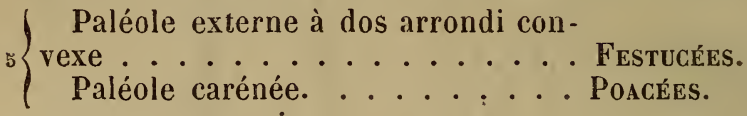

ORDRE DE SUCCESSION DES SOUS-TRIBUS.

10 Cynosurées.

$2^{\circ}$ Festucées.

$3^{\circ}$ Poacées.

$4^{\circ}$ Arundinées.

5" Triodiées.

6. Seslériées.

Ire sous-tribu. - Cynosurẻes.

Panicule spiciforme, unilatérale; locustes comprimées, les unes fertiles et les autres réduites à une bractée pinnée ou pectinée; paléole externe fortement comprimée, carénée.

\section{GENRE 10. - GyNOSURE (CYNOSURUS L.).}

Locustes de deux à cinq fleurons comprimés latéralement, dont quelques-uns munis à leur base d'une locuste stérile ressemblant à une bractée pinnée ou pectinée. Paillettes linéaires-lancéolées, égales, acuminées ou aristées; paléole extérieure mucronée ou aristée au sommet, l'intérieure bicarénée, bifide au sommet; paléolules 2 , entières ou lobées; ovaire turbiné, glabre; stigmates 2, 
DEUXIĖNE PARTIE. - ANALYSES ET DESCRIPTIONS. 10\% réfléchis, plumeux, terminant des styles exirêmement courts ; grain oblong, comprimé, acuminé aux deux extrémités; paléoles ne se détachant point du

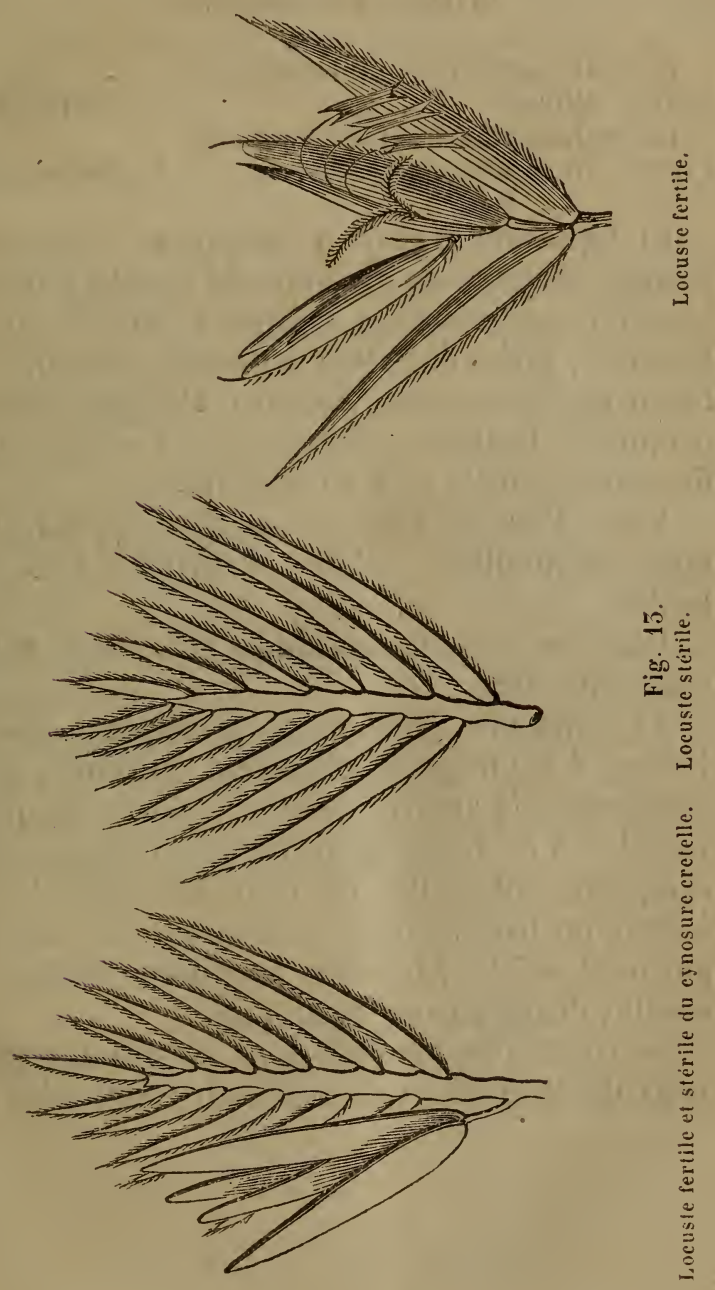


grain par le battage; panicule spiciforme compacte, unilatérale, allongée, linéaire-ovoïde ou subcapitée.

ANALYSE DES ESPÈCES.

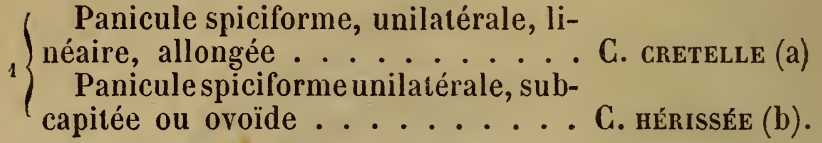

a) Cynosure cretelle. (Cynosurus cristatus L.) Souche cespiteuse; chaumes de quatre à neuf décimètres, peu robustes et dressés; feuilles étroites, linéaires, pliées dans leur jeunesse; panicule spiciforme et racémiforme, linéaire, allongée; bractées pectinées; locustes pubescentes à trois ou quatre fleurons ; paléole externe acuminée.

Var.) Paléoles allongées et développées en manière de feuilles. Vivace; fleurit en juin et en juillet.

Dans les prés, les pâturages et les lieux tant humides que secs.

b) Cynosure hérissé. (Cynosurus échinatus Dmtr.) Chaumes ordinairement solitaires, ou peu nombreux, de quatre à neuf décimètres, un peu genouillés à la base; panicule spiciforme, courte, compacte, subcapitée ou ovale-oblongue; locustes à deux ou trois fleurons; paléole externe assez longuement aristée; bractées pectinées, aristées. Annuelle; fleurit en mai et en juin.

Se trouve quelquefois dans les champs sablonneux du Hainaut el de la Flandre orientale; rare. 


\section{ne sous-tribu. - Festucées.}

Panicule plus ou moins rameuse; locustes ordinairement toutes fertiles; paléole externe à dos arrondi, très-rarement à peine carénée.

GENRe 11. - FÉtuQue (Festuca L.).

Locustes comprimées latéralement, de trois à quinze fleurons; paillettes herbacées ou membraneuses, carénées, mutiques ou aristées, inégales, l'intérieure rarement deux à dix fois plus courte, quelquefois presque égales; paléoles presque égales, l'intérieure entière non carénée ou seulement carénée au sommet, munie d’une arête plus ou moins longue, rarement mutique; paléole intérieure bicarénée, émarginée, bifide ou bidentée; paléolules; 2, ovales, entières et velues; une ou trois élamines; ovaire turbiné; styles courts, terminaux; stigmates 2, plumeux; grain oblong, acuminé aux extrémités, parcouru par un sillon et étroitement renfermé entre les paléoles et les paléolules; panicule resserrée ou élalée à locustes plus ou moins longuement pédonculées.

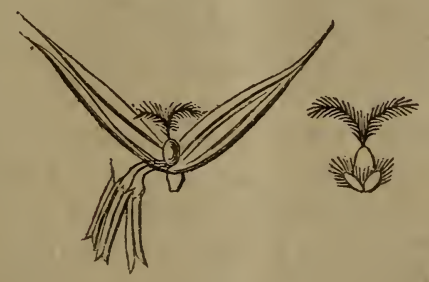

Fig. 11

Fleuron de fetuque. Ovaire et paleolule. 


\section{ANALYSE DES ESPÈCES.}

(Arête terminale plus longue que la paléole.

1 Arête terminale plus courte que la paléole ou arête subterminale de longueur variable.

4.

Paillette externe très-courte, squamiforme, obtuse ou nulle, l'interne

2 aristée neuf ou dix fois au moins plus grande.

Paillettes aiguës.

Paillette extérieure ayant environ le (tiers de la longueur de l'interne; pa5 nicule ordinairement engaînée.

5) Paillette extérieure de moitié plus courte que l'intérieure; panicule ordi- FétuQue QUEUE nairement libre. ....... D'ÉcureurL (c).

Arête subterminale deux ou trois fois ${ }_{4}$ plus longue que la paléole. . . . Fétuque géant (n).

Arête n'atteignant jamais cette lon-

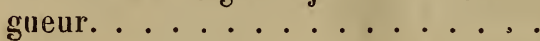

Ligule oblongue obtuse; chaumes FÉtuQue Calamare s entourés à la base d'écailles brunes. .

Pas de ligule oblongue. . . . . . (m).

Ligule biauriculée; gaîne non biauriculée supérieurement; feuilles radicales très-étroites pliées ou enroulées.

6 Ligule non biauriculée; gaine souvent biauriculée supérieurement;feuilles minces, les radicales assez larges, planes. ...........

Fétuque Quede de SOURIS (b).

Souche émettant des rhizomes lon${ }_{7}\left\{\begin{array}{l}\text { guement traçants. . . . . . . . } \\ \text { Souche cespiteuse non traçante. }\end{array}\right.$ FétuQue rouge (h).

Feuilles caulinaires planes, les au- F. IÉTÉnopiytle ${ }_{8}$ tres plus ou moins enroulées. . . . (e).

Feuilles caulinaires plus ou moins enroulées ou pliées.

F. polymoritie (d). 
DEUXIĖUE PARTIE. - ANALYSES ET DESCRIPTIONS. 109

Locustes supérieures sessiles, les autres très-courtement pedonculées (cette

9 plante au premier aspect rappelle l'ivraie). . ............. F. Fausse ivraie (i).

Panicule rameuse. ...... 10.

Rameaux inférieurs géminés, le rameau le plus court portant le plus souvent une locuste; l'autre 3-5 locus- Fétuque des prés tes contenant $6-10$ fleurons. : ... (k).

Rameaux inférieurs géminés ou solitaires formant deux divisions portant chacune $\check{b}$ à 30 locustes à $4-6$ fleurons............... roseau (I).

$\S$ I. Arète terminale plus longue que la paléole; une étamine; plantes n'émettant pas de fascicules de feuilles stériles.

a) Fétuque à une paillette. (Festuca uniglumis Host.) Chaume glabre, grêle, un peu couché et feuillé dans le bas, nu et redressé dans le haut; feuilles enroulées, sétacées; panicule resserrée en forme d'épi unilatéral ; locustes à 5-6 fleurons ; paillette externe très-courte, squamiforme, obtuse ou nulle; l'interne aristée, neuf ou dix fois plus grande. Annuelle; fleurit en mai et en juin.

Dans les terrains arénaires du Hainaut; rare(?).

b) Fétuque queue de souris. (Festuca pseudomyuros, Soy-willmt.) Chaumes coudés inférieurement; feuilles enroulées, sétacées; panicule rameuse allongée, un peu penchée et ordinairement embrassée par la gaìne de la feuille supérieure; paillettes carénées, aiguës, l'extérieure n'égalant pas la moitié de la paillette intérieure, à peine longue d'un millimètre; l'autre longue de quatre 
millimètres; locustes à 2-כ̆ fleurons. Annuelle; fleurit en juin et en juillet.

Se trouve fréquemment avec la suivante.

c) Fétuque queue d'écureuil. (Festuca sciuroides Roth.) Chaumes nombreux, droits, nus supérieurement; feuilles enroulées, sétacées; panicule unilatérale courte, droite, presque simple, un peu étalée pendant la floraison; locustes de 5-6 fleurons; paillette extérieure de moitié plus courte que l'intérieure : celle-ci a sept millimètres de longueur et l'autre n'en a que quatre environ.

Il y en a une variété à panicule rameuse à la base. Annuelle; fleurit en juillet.

Se montre dans les endroits secs, sablonneux, sur les bords des chemins.

$\S$ II. Arête terminale plus courte que la paléole, quelquefois nulle; feuilles pliées, carénées ou enrouléessétacées, au moins les radicales; ligule biauriculée; souche émettant un grand nombre de fascicules de feuilles stériles.

d) Fétuque polymorphe. (Festuca polymorpha Nob.) Souche cespiteuse; chaumes de deux à six décimètres, grêles; feuilles enroulées, sétacées. mème les caulinaires, très-fines, scabres ou lisses; panicule dressée, serrée, étroite, convexe, unilatérale; locustes verdâtres ou violacées, oblongues, à 3-6 fleurons, glabres ou pubescentes; paléole courtement aristée ou mutique.

Cette plante, riche en variétés, présente deux races distinctes: dans l'une, les feuilles sont scabres au dos; dans l'autre, les feuilles sont à dos lisse. La première race présente deux variétés remar- 
quables par leurs qualités opposées : c'est la fétuque des brebis aristée, qui plait beaucoup aux moutons, et la fétuque des brebis capillaire mutique, connue encore sous le nom de festuca tenuifolia, dont les moutons se montrent peu friands.

La seconde race comprend aussi deux variétés assez stables qui sont :

$1^{\circ}$ La fétuque durette (Festuca duriuscula L.), ainsi caractérisée : souche cespiteuse; chaumes de 2-6 décimètres, assez roides; feuilles plus ou moins roulées ou pliées, étroites, lisses ou scabriuscules; les radicales souvent arquées et formant des touffes denses; panicule dressée â rameaux droits ou étalés, ordinairement unilatérale; locustes verdàtres ou violacées, oblongues, à $4-7$ fleurons, glabres, pubescentes ou velues; paléole externe lancéolée, aristée, rarement mutique;

$2^{\circ} \mathrm{La}$ fétuque glauque.(Festuca glauca Schrad.) Cette plante rappelle assez bien la fétuque durette dont elle ne diffère que par ses feuilles tout à fait lisses au dos et par son aspect d'un glauque blanchâtre, comme si la plante avait été saupoudrée de farine. Vivace; fleurit de mai à juillet.

Les deux premières se trouvent dans les lieux arides, sur les bords des bois; les autres recherchent les rochers calcaires et les sols arides.

e) Fétuque héterophylle. (Festuca heterophylla Lam.) Souche cespiteuse; feuilles nombreuses, les radicales enroulées ou subcarénées, plus ou moins rudes, formant des touffes denses, d'où s'élèvent des chaumes de cinq à sept décimètres, munis de quelques feuilles à limbe plus large que celui des radicales et plane; panicule unilatérale làche, à rameaux inférieurs longs, étalés, les supérieurs presque dressés; locustes ordinairement verdâtres, 
oblongues, à 4-7 fleurons, glabres ou légèrement pubescentes; paléole externe lancéolée, aiguë, aristée, à arête plus courte que la paléole. Vivace; fleurit en juin-juillet.

Endroits humides etcoteaux secs, ombragés ; pas rare dans les haies et les taillis.

f) Fétuque rouge. (Festuca rubra L.) Souche émettant des rhizomes longuement traçants d'où sélèvent de distance en distance des touffes de feuilles peu serrées; les feuilles sont plus ou moins enroulées, très-rarement presque planes; chaume de un à neuf décimètres de hauteur; feuilles caulinaires presque planes ou enroulées; panicule dressée, à rameaux plus ou moins étalés, roides, rarement faibles et presque penchés; locustes verdâtres-violacées ou jaunâtres, oblongues, à 4-7 fleurons; paléole externe lancéolée, aiguë, aristée, à arête moins longue que le fleuron.

II en existe plusieurs variétés dont quelquesunes se font remarquer par leur vigueur et leur développement : elles habitent les dunes et les sables maritimes. L'une (F. cinerea D.C.) a des feuilles épaisses, subcharnues, à face inférieure glauque et à face supérieure ou intérieure glauque-cendré, pubescente et à locustes pubescentes veloutées; l'autre qui a le mème port s'en distingue par ses locustes glabres.

$\S$ III. Arête subterminale ou nulle; feuilles planes, même les caulinaires; ligule non biauriculée; souche émettant un grand nombre de fascicules de feuilles stériles.

i) Fétuque fausse ivraie. (Festuca loliacea IIuls.) Souche subcespiteuse-traçante; chaumes 
feuillés de 6-12 décimètres; feuilles linéaires-lancéolées; locustes au nombre de douze à quinze, oblongues-linéaires, solitaires et disposées à peu près sur deux rangs comme les ivraies; locustes inférieures légèrement pédonculées, quelquefois

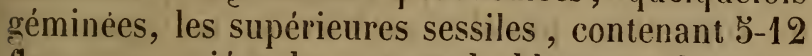
fleurons, variées de vert et de blanc ou de pourpre; paléoles externes un peu pointues, l'intérieure presque glabre; paillettes striées, l'extérieure à cinq nervures et l'intérieure à une nervure. Vivace; fleurit en juin.

Prairies humides.

k) Fétuque des prés. (Festuca pratensis Huds.) Souche subcespiteuse ; chaumes droits ou un peu courbés, ou coudés inférieurement; feuilles planes; ligule très-courte; panicule plus ou moins ramifiée, étalée, et plus ou moins unilatérale; rameaux inférieurs géminés, le rameau le plus court portant le plus souvent une locuste, l'autre $5-5$ locustes ; rameaux supérieurs solitaires ; locustes ordinairement d'un vert rougeâtre, à 4-10 fleurons; paléoles extérieures obtuses, un peu blanchàtres et scarieuses en leurs bords à cinq nervures, quelquefois pourvues d'une petite arête au-dessous du sommet. Vivace; fleurit en juillet.

Prés fertiles.

Cette espèce présente plusieurs variétés qui ne diffèrent entre elles que par leur vigueur, leur élévation et leur succulence : la plus grande, quoique assez grossière, fournit la plus grande masse de fourrage et de meilleure qualité; c'est aussi cellelà qu'il faut choisir pour la formation du mélange destiné à l'emblavure des prairies.

l) Fétuque roseau. (Festuca arundinacea Schreb.) Souche subcespiteuse; chaumes forts, GR:MINÉES. 
roides, un peu coudés à leur base; feuilles longues et larges; ligule très-courte; panicule à rameaux inférieurs géminés, ramifiés, rudes, portant 5 à 30 locustes souvent très-rapprochées, ovales-lancéolées, à 4-ว̌ fleurons verdâtres, ou légèrement panachées de rougeâtre; paléoles extérieures mutiques ou munies d'une petite arête. Vivace; fleurit en juillet.

Abonde sur les bords des rivières et dans les prairies humides à sol salin.

m) Fétuque calamaire. (Festuca calamaria Schreb.) Chaumes droits, arrondis, lisses, entourés, à leur base, d'écailles brunes, ainsi que les faisceaux de feuilles qui les accompagnent; feuilles planes, les caulinaires linéaires-lancéolées, assez longues, glabres, d'un vert glauque en dessus; ligule oblongue, obluse; panicule droite, làche et étalée pendant la-floraison, puis resserrée et penchée ; locustes oblongues à $3-6$ fleurons; paléole extérieure lancéolée, très-aiguë, à 3 nervures scabriuscules. Vivace; fleurit en juin et en juillet.

Endroits humides, ombragés, des bois montagneux des provinces de Liége et de Luxembourg.

n) Fétuque géant. (Festuca gigantea Vill.) Souche subcespiteuse; chaume de 6 à 12 décimètres, penché au sommet; feuilles grandes, assez longues et larges; ligule très-courte; panicule trèsétalée, làche, penchée au sommet; rameaux inférieurs le plus souvent géminés; locustes lancéolées à 丂̆-10 fleurons; paléole extérieure munie d'une arête plus ou moins ondulée, environ deux ou trois fois plus longue que la paléole. Vivace; fleurit de juin à août.

Endroits humides, ombragés, dans les bois et les haies. 
DEUXIEME PARTIE. - ANALYSES ET DESCRIPIONS. II;

GENRE 12. - BRONE (BROMUS L.)

Locustes comprimées latéralement, à 4-20̆ fleurons; glume à paillettes plus ou moins herbacées ou membraneuses, souvent carénées, mutiques, inégales; paléole extérieure herbacée, convexe, légèrement carénée supérieurement, ou souvent bidentée ou bifide, quelquefois entière, mutique, ordinairement munie d'une arête naissant au-dessous du sommet ou dans l'échancrure qu'elle présente, jamais tordue; paléole intérieure scarieuse, bicarénée, glabre, pubescente ou ciliée, émarginée ou bidentée, souvent un peu roulée; paléolules 2 , obovales, entières; ovaire turbiné, hérissé au sommet, quelquefois glabre; styles latéraux; grain oblong, linéaire, à dos convexe, à face interne canaliculée, enveloppé par les paléoles; panicule simple ou rameuse.

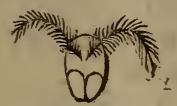

Fig. 13.

Pistil d'un brome.

ANAIÝSE DES ESPÈCES.

Paléole interne non ciliée ou à peine ciliée-pubescente; plantes viraces.

Paléole interne ciliée on pectinée; plantes annuelles ou bisannuelles. .

2.

4.

Feuilles pliées dans leur jeunesse. Brome dressé (b)). Feuilles enroulées dans leur jeunesse. ............. 
Rameaux floraux ordinairement disposés par 2-3; arête de moitié plus courte que la paléole. ....... Brone rude (c).

3 Rameaux floraux ordinairement disposés par 5-\$; arête nulle ou trèscourte, n’atteignant jamais la moitié de la largeur de la paléole ..... Brone inerme (a).

Arête subtrigone à la base, canaliculée à sa face supérieure; paillette externe à une nervure, l'interne à trois nervures. ..........

Arête arrondie, rarement nulle par avortemeht; paillette externe à cinq nervures ou plus. ........

Paniculedressée, plus ou moins res.

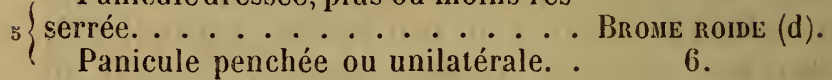

Rameaux de la panicule scabres,

porlant la plupart 1-2 locustes. . . Brone stérile (e).

6 Rameaux de la panicule doux au toncher ou à peine scabres, portant 4

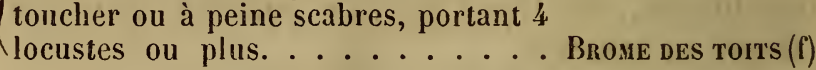

Paléole triaristée, munie de chaque

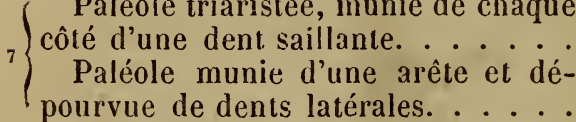

Brome Michel (i).

Paléole intérieure des fleurons supérieurs de chaque locuste dépassant

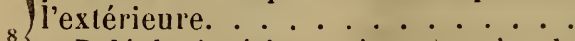

Paléole intérieure à peu près de même longueur ou sensiblement plus courte que l'extérieure. . . . . . 5.

7.

8.

9.

10.

(Gaînes inférieurescouvertes de poils courts qui forment un duvet cotonneux

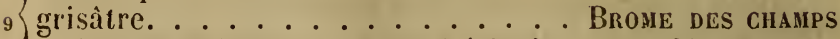

Gaînes glabres ou à peine hérissées

de quelques poils. ....... Brome seiglin (I).

10 Gaînes glabres ou presque glabres.

${ }^{10}$ Gaines pubescentes ou poilues. .

11.

12.

11 Locustes pubescentes cotonneuses. Brome ÉPAIs (j),

11 Locustes glabres, presque luisantes. B. Luisant (k). 
DUUXIEME PARTIE. - ANALYSES ET DESCRIPTIONS. 117

Panicule simple ou presque simple, à rameaux longs, penchés, à locustes pendantes après la floraison; pa. léole externe ovale-elliptique, échan-

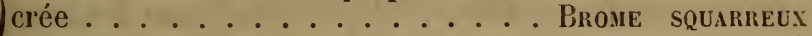

Plantes ne réunissant pas ces caractères. ..............

Chaume glabre, lisse ou scabre sous

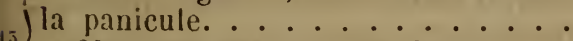
(g). 15.

\section{4.}

Chaume mollement pubescent ou poilu sous la panicule. . . . . Brome nou (o).

Paléole externe des fleurous supérieur's de chaque locuste à bords étendus, présentant un angle obtus vers son quart supérieur; paléole interne de tous les fleurons sensiblement plus

14 courte que l'externe ....... Brome échangé(n).

Paléole exterue des fleurons supérieurs à bords étendus, scarieux, ovalaires; paléole interne des fleurons supérieurs de mème longueur ou à Brone EN GRAPPE peine plus courte que l'externe. ... (m).

$\S$ I. Paléole externe à peine ciliée-pubescente; plantes vivaces.

a) Brome inerme. (Bromus inermis L.) Souche cespiteuse-subtraçante; chaumes droits de 7 a 11 décimètres; feuilles larges, glabres ou hérissée: de quelques poils, lisses, enroulées dans leur jeunesse; ligule courte; panicule droite, d'abord resserrée, un peu étalée à la floraison; rameaux. verticillés par $4-8$; locustes linéaires-lancéolées à วั-10 fleurons; paléole extérieure obtuse ou légèrement bidentée, mutique, ou munie d'une petite aréte au-dessous du sommet ou dans une échancrure qu'elle présente quelquefois. Vivace; fleurit en juin et en juillet.

Prés et hois avoisinant la Meuse. 
b) Brome dressé. (Bromus erectus Huds.) Souche cespiteuse; chaume de 9 à 15 décimètres; feuilles radicales disposées en gazon, très-étroites, ordinairement carénées, ciliées, ainsi que les caulinaires qui sont deux à trois fois plus larges que les radicales et pliées dans leur jeunesse; ligule courte; panicule égale, roide, à rameaux disposés inférieurement par 2-6, dressés ; locustes linéaires-lancéolées; paléole extérieure à $\breve{-7}$ nervures, légèrement bidentée au sommet; arête de moitié plus courte que la paléole, insérée dans l'échancrure qu'elle présente. Vivace; fleurit en juin et en juillet.

Bords des champs et prés secs.

c) Brome rude. (Bromus asper L.) Souche cespiteuse; chaumes peu nombreux de 8 à 19 décimètres, durs, velus; feuilles larges, planes, presque glabres ou pubescentes en dessus, légèrement velues en dessous; gaînes recouvertes de longs poils un peu roides, dirigés par en bas; panicule rameuse, à rameaux inférieurs ordinairement géminés, très-allongés, penchés; locustes linéaires-lancéolées à 7-26 fleurons; paléole extérieure à 3 nervures, légèrement bidentée, portant une arête droite de moitié environ plus courte que la paléole. Vivace; fleurit en juin et en juillet.

Dans les bois montagneux et couverts et dans les buissons.

$\S$ II. Paléole interne ciliée-pectinée; plantes annuelles ou bisannuelles.

A. - palllette externe $a$ une nervure, L'interne A troIs NERVURES.

d) Brome roide. (Bromus rigidus Roth.) Chaume roide, droil, de 5 à 7 décimètres, glabre; 
feuilles planes, étroites, pubescentes, un peu rudes; gaines pubescentes; panicule simple, rarement rameuse, droite, un peu resserrée, à lameaux droits pubescents, lisses ou à peine scabres, rudes; locustes ovales, rudes, à 5้-11 fleurons; arêtes presque terminales, droites, plus longues que la paléole. Annuel; fleurit en juin el en juillet.

Prés secs de la province de Luxembourg.

e) Brome stérile. (Bromus sterilis L.) Chaumes presque droits, de 5 à 10 décimètres, glabres, nus dans le haut; feuiles larges, planes, velues, un peu rudes; panicule làche, étalée, à rameaux (les inférieurs sont quelquefois divisés et portent כ̋-1丂 locustes) longs, menus, scabres, penchés, portant la plupart 1-2 locustes comprimées, oblongues, élargies au sommet après la floraison par la divergence des fleurons qui sont au nombre de ऽ-9; celles-ci distiques, écartées; arêtes presque terminales, droites, plus longues que la paléole. Annuel et bisannuel; fleurit de juin à septembre.

Croît dans les champs et les lieux incultes, les haies et au pied des murs.

f) Brome des toits. (Bromus tectorum L.) Chaumes droits, de 5 à 6 décimètres, grêles, ordinairement pubescents dans leur partie stiperieure; feuilles assez larges, planes, pointues, le plus souvent hérissées, ainsi que les gaines, de poils mous, dont la plante se dépouille quelquefois par l'àge, rarement glabre; panicule rameuse, étalée, un peu penchée au sommet, presque unilatérale; rameaux doux au toucher ou à peine scabres, grèles, verticillés, portant $14-\breve{3}$ locustes ou plus; locustes linéaires, élargies au sommet, pubescentes; fleurons imbriqués; arête presque terminale, à peine ṕlus 
longue que la paléole. Annuel; fleurit de mai à juillet.

Dans les endroits sees, au pied des murs, dans les décombres, les lieux arides et stériles.

\section{B. - Palllette interne a 5-5 Nervures ou plus.}

g) Brome squarreux. (Bromus squarrosus L.) Chaume grêle, de 3 à $\breve{~ d e ́ c i m e ̀ t r e s ; ~ f e u i l l e s ~ e ́ t r o i t e s, ~}$ molles et pubescentes sur les deux faces; gaine velue; panicule simple, lâche, étalée, penchée avant et après la floraison; locustes ovoïdes-lancéolées, à 6-18 fleurons, à paléole externe ovale-elliptique, échancrée; fleurons rapprochés sur deux rangs et munis d'une arête assez forte qui diverge plus ou moins à mesure que la maturité avance; paléole interne beaucoup plus courte que l'externe; la plus grande paillette à $\breve{5}-9$ nervures. Annuel; fleurit en juin.

Dans les moissons : rare.

h) Brome des champs. (Bromus arvensis L.) Chaumes nombreux, de 7 à 11 décimètres, droits, glabres, lisses; feuilles et gaînes poilues; gaînes inférieures couvertes de poils courts qui forment un duvet cotonneux, grisâtre; panicule ouverte, droite avant la floraison, penchée lors de la maturité; rameaux de la panicule très-longs, les inférieurs souvent six à dix fois plus longs que les locustes qui sont linéaires, étroites, à $\breve{-10}$ fleurons elliptiques, lancéolés, imbriqués; paléole interne des fleurons supérieurs dépassant l'externe, la plus grande à 5 nervures; arête droite de même longueur environ que la paléole. Annuel; fleurit en juillet et en août.

Dans les moissons et les prés. 
i) Brome michel. (Bromus michelianus Nob. Bromus triaristatus Lois. Libertia arduennensis Lej. Michelaria bromoïdea Dmtr.) Souche cespiteuse; chaume droit, de cinq à onze décimètres, simple, lisse, glabre, strié, nu ou calleux sous la panicule; nouds légèrement velus, luisants et jaunâtres inférieurement; gaînes glabres plus courtes que le limbe; ligule ovale, lacérée; feuilles à sept ou neuf nervures, glabres en dessous, scabres en dessus ; axe terminal continu, articulé, paniculé ; panicule presque simple, un peu penchée; rameaux scabres; locustes obovales, scabres, de sept à dix fleurons distiques; paléole externe auriculée vers le milieu de ses bords (présentant de chaque côté une dent) et munie de trois arêtes terminales dont celle du milieu deux fois environ aussi longue que les latérales; paléole interne un peu plus courte que l'externe. Annuel; fleurit en juin et en juillet.

Se trouve dans les moissons de quelques localités de la province de Liége; se rencontre aux environs d'Aiwaille, Comblain-au-Pont; dans la province de Namur, aux environs de Dinant et de Rochefort.

j) Brome épais. (Bromus grossus DC.)Chaume droit, de sept à onze décimètres, glabre; feuilles larges, planes, glabres ou hérissées de quelques poils blancs; gaînes des feuilles glabres; panicule étalée, penchée après la floraison; locustes pubescentes, grisàtres, peu nombreuses, oblongues-lancéolées, de neuf à vingt fleurons; arètes de la longueur des paléoles, droites ou presque droites; la plus grande pailletteà sept ou neuf nervures; fleurons devenant distinctes à la maturité et laissant paraittre la scobine; paléole interne sensiblement plus courte que 
l'externe; locustes quelquefois vivipares. Annuel; fleurit en juillet et en août.

Dans les champs secs.

k) Brome luisant. (Bromus nitidus Dmtr.) Chaumedroit, de sept à dix décimètres, glabre, lisse; feuilles étroites, planes, pointues, hérissées de quelques poils longs et soyeux; gaines inférieures glabres; panicule longue, làche, penchée, peu garnie, à rameaux grêles, rudes, fort longs, inégaux et verticillés; locustes ovales-lancéolées, glabres, luisantes; fleurons imbriqués; arête droite; paléole externe un peu plus courte que l'interne. Annuel; fleurit en juin et en juillet.

Dans les moissons et les prés des Ardennes.

l) Brome seiglin. (Bromus secalinus L.) Chaume droit, de 9 à 13 décimètres, glabre, lisse; feuilles larges, planes, glabres en dessus, chargées en dessous de quelques poils épars ; gaînes glabres, striées; panicule penchée, à rameaux inégaux, disposés par 3-7 et formant un verticille incomplet, les inférieurs ne dépassant pas la plupart quatre fois la longueur de la locuste; locustes glabres ou pubescentes, à 5 -9 fleurons imbriqués et espacés à la maturité; paléole intérieure dépassant l'extérieure; la plus grande paillette présentant 7-9 nervures; arêtes ordinairement flexueuses, droites, plus courtes que la paléole. Annuel; fleurit de mai à juillet.

Se rencontre dans les moissons et les prés.

m) Brome en grappe. (Bromus racemosus L.) Chaume droit, de 6 à 11 décimètres, glabre, lisse; feuilles planes, glabres ou hérissées de quelques poils; gaînes des inférieures velues; panicule droite ou légèrement penchée, contractée ou étalée après la floraison, à rameaux simples ou divisés ; 
locustes ovales-oblongues, glabres ou presque glabres, à ว̆-10 fleurons ou plus, imbriqués, ordinairement d'un vert pâle; paléole externe des fleurons supérieurs de chaque locuste à bords scarieux, étendus, ovalaires, à nervures peu saillantes; palénle interne des fleurons supérieurs un peu plus courte que l'externe, celle des fleurons supérieurs à peine plus courte ou de mème longueur que celle-ci ; arête presque aussi longue qu'elle; la plus grande paillette à 7-9 nervures. Annuel; fleurit de mai à juillet.

Dans les moissons et sur les bords des prairies.

n)Bromeéchangé.(Bromus commutatus Schrad.)

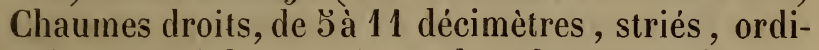
nairement glabres ou à nœuds pubescents; feuilles planes, molles et velues, ainsi que leurs gaînes, notamment les inférieures; panicule lâche, un peu étalée, penchée au sommet après la floraison, à rameaux, les uns simples, les autres divisés ; locustes lancéolées, oblongues, glabres, un peu comprimées, de 7 à 13 fleurons oblongs-elliptiques, serrés et se recouvrant les uns les autres, ordinairement d'un rouge violacé sur la face exposée aux rayons du soleil; paléole extérieure dépassant sensiblement l'intérieure; celle des fleurons supérieurs de chaque locuste à bords étendus présentant vers leur quart supérieur un angle très-marqué et à nervures peu saillantes : cet angle existe au-dessus du milieu ou vers le tiers supérieur dans les fleurons inférieurs. Annuel; fleurit de mai à juillet.

Dans les moissons et les prés.

Quelques auteurs ont encore décrit comme espèce belge le brome étalé de M. et K. Les échantillons qu'on nous a communiqués des Ardennes, sous ce nom, constituent une simple variété du 
brome échangé à arêtes divergentes. Or, la divergence des arêtes est un caractère de trop peu d'importance pour qu'on s'y arrête. En effet, les dunes nous offrent diverses variétés debromes, enlreautres le brome mou à arêtes divergentes, réfléchies, qui, semés dans une terre fertile, reproduisent le brome mou à arêtes droites. Lebrome squarreux, que nous considérons comme une espèce exotique, ne présente presque jamais dans notre pays des arêtes divergentes réfléchies. Le brome en grappe et le brome échangé ont entre eux beaucoup d'analogie et ne sont apparemment que des variétés d'une même espèce; l'angle que présente la paléole externe du brome échangé en constitue le principal caractère diagnostique.

o) Brome mou. (Bromus mollis L.) Chaume droit, de 4 à 7 décimètres, glabre dans le bas, velouté, ou velu sous la panicule; feuilles planes, pointues, assez larges, couvertes, ainsi que les gaînes, surtout celles des feuilles inférieures, de poils mous, blanchâtres; panicule droite, rameuse ou simple, plus ou moins resserrée; rameaux plus ou moins nombreux, inégaux ; locustes ovales, à 丂̆-11 fleurons, pubescentes; paléole externe fortement nerviée à la maturité, l'interne sensiblement plus courte que l'externe; arête presque aussi longue que la paléole, la plus grande paillette à 7-9 nervures. Annuel; fleurit en juin.

Sur les bords des prairies et le long des chemins. 
GeNre 15. - ÉNodie (ENodidi P. D. B.)

Locustes subconiques à 5 -ə̆ fleurons, le supérieur au moins stérile; paillettes un peu carénéesconvexes, mutiques, inégales; paléoles mutiques presque égales, l'extéricure convexe, demi-cylindrique à 5̆ nervures, l’intérieure bicarénée, émarginée; paléolules 2, obovales, libres, obliquement tronquées; ovaire glabre; styles allongés; stigmates 2, plumeux; grain oblong, cylindrique, présentant un sillon étroit; panicule spiciforme ou étalée; chaume présentant 1 à 8 nouds très-rapprochés à la base, où s'insèrent les feuilles au nombre de 2 à 6 ; gaîne de la feuille inférieure recouvrant les nœud. el les gaines des autres feuilles.
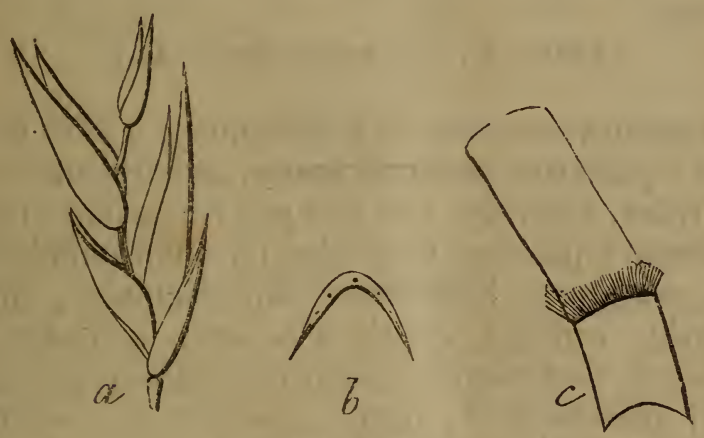

Fig. 16 (1).

Énodie bleue. (Enodium cœruleum P. D. B.) Souche cespiteuse à racines robustes, dures, d'un blanc jaunâtre; bases des feuilles des années précédentes, jaunâtres ou brunâtres; chaumes de 5

(1) a Locuste d'enodie bleue; $b$ coupe de la palcolc externe; portion de feuille avec la ligule pilcuse. 
à 13 décimètres, droits, glabres, lisses ou un peu rudes, n'ayant le plus souvent qu'un seul noud très-prononcé, placé à la base; feuilles glabres ou un peu velues, étroites ou assez larges, pointues ou émoussées; la supérieure à gaîne fort longue, striée, atteignant souvent plus de la moitié de la longueur du chaume; ligule pileuse ; panicule étalée ou resserrée, un peu régulière ou unilatérale; locustes à 2-6 fleurons, très-rarement uniflores.

Dire qu'il n'y a pas deux pieds qui se ressemblent entièrement, c'est exposer l'impossibilité où l'on se trouve de découvrir des caractères qui fassent reconnaitre les nombreuses variétés que présente l'énodie bleuâtre. Vivace; fleurit de juillet à septembre.

Bois taillis, bruyères, lisières des bois, sapinières.

$$
\text { GENRE 14. - brize (briza L.) }
$$

Locustes comprimées latéralement, à 4-30 fleurons; paillettes presque égales, suborbiculaires, convexes, ventrues, tant soit peu comprimées latéralement; paléoles herbacées ou submembraneuses, mutiques; l'extérieure suborbiculaire, comprimée, convexe, cordée à la base, arrondie au sommet; l'intérieure bicarénée, beaucoup plus petite; paléolules 2, libres, entières ou lobées; ovaire arrondi, glabre; styles 2 , courts, terminaux; stigmates 2 , plumeux, glanduleux; grain comprimé, terminé par deux petites pointes filiformes; panicule étalée ou engaince à la base; locustes souvent penchées, mobiles. 

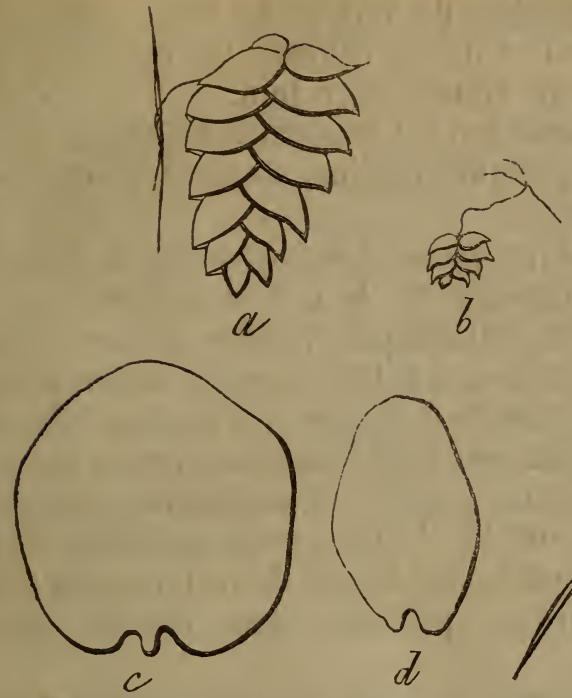

Fig. 17 (1).

ANALYSE DES ESPÈCES.

Plante annuelle. .......

Plante vivace; panicule rameuse;

ligule courte........... Beize moyenne (b).

Panicule simple. ...... Brize a gros EPIL-

Panicule rameuse; ligule longue, LETs (a).

lancéolée. ..................

a) Brize à gros épillets. (Briza maxima L.) Chaume ordinairement rameux, de 3 à J̆ décimètres, garni de deux à quatre feuilles linéaires, planes, glabres; panicule simple, étalée, composée de 2 à 11 locustes, pendantes, contenant $\breve{~ a ̀ ~} 50$ fleurons,

(1) a Locuste de brize à gros ćpillets ; b locuste de la brize moyenne ; $c$ paléole externe étendue; $d$ palćole externe vue de profil; $c$ coupe de la paléole externe. 
d'abord panachés de vert et de blanc, puis d'un blanc roussâtre ou de couleur ferrugineuse. Annuelle; fleurit en mai et en juin.

Cette plante qui est originaire d'Espagne et cultivée dans quelques jardins, se montre rarement à l'état sauvage.

b) Brize moyenne. (Briza media L.) Souche traçante; chaume de 4 à 9 décimètres, droit, glabre, lisse, cylindrique ; feuilles glabres, larges, planes, pointues, à gaînes presque aussi longues que les mérithalles; ligules très-courtes, obtuses; panicule rameuse, étalée ou roussàtre, à rameaux souvent géminés, grëles et capillaires, assez fréquemment ondulés ; locustes assez nombreuses, verdâtres, panachées de blanc et de vert, ou rougeâtres, à 4-8 fleurons; paillettes plus courtes que la locuste.

On en trouve une variélé remarquable panicule resserrée et engainée que quelques auteurs considèrent comme le Briza virens de Linné. Vivace; fleurit en juillet et en aoùt.

Au bord des bois, dans les prés secs et tourbeux.

c) Brize petite. (Briza minor L.) Chaume de 2 à 6 décimètres, souvent rameux, un peu étalé à la base; feuilles linéaires-lancéolées, à bords rudes; ligule très-longue, lancéolée, entière ,ou lacérée; panicule étalée ou engainnée, à rameaux flexueux; paillettes de même longueur environ que la locuste composée de 4 à 7 fleurons verdâtres ou purpurescents. Annuelle; fleurit en mai et juin.

Aux bords des bois, dans les terres sablonneuses. Rare. 
GenRe 1ว̆. - Glycérie (GLyceria R. BR.)

Locustes plus ou moins comprimées latéralement, à 5 -15 fleurons libres; paillettes presque membraneuses; paléoles subherbacées, mutiques, presque égales en longueur, l'extérieure convexe, demi-cylindrique, obtuse, arrondie et scarieuse au sommet, à 5-7 nervures, l'intérieure bicarénée, entière ou émarginée; paléolules 2 ; ovaire glabre; styles terminaux un peu allongés, à base persistante ; stigmates 2, plumeux; grain oblong, sillonné d'un côté, souvent libre; panicule rameuse ou presque simple, assez souvent unilatérale, rarement ample, régulière.
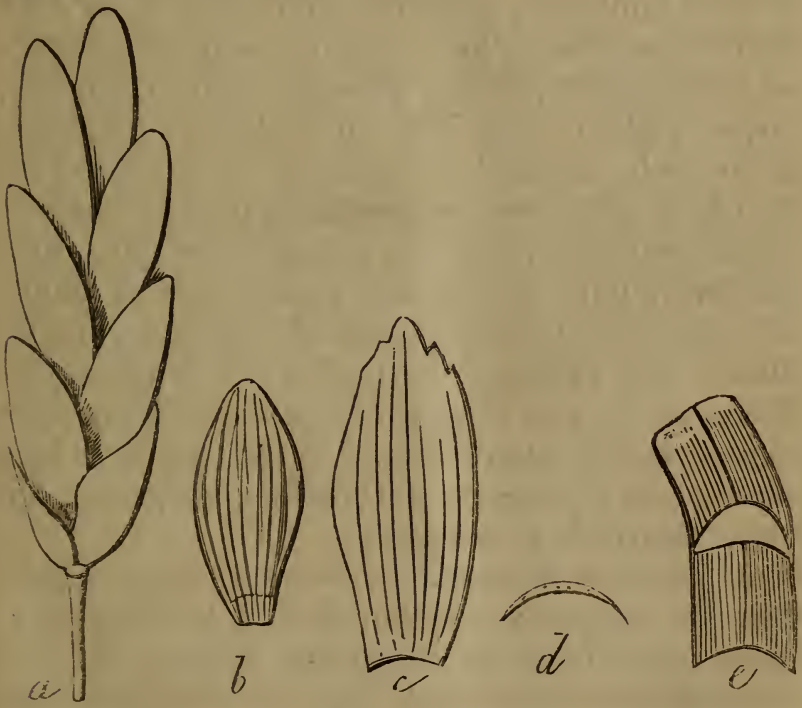

Fig. 18 (1).

(1) a Locuste de glycérie élevée, grossie ; $b$ paléole extcrne de la mème; $c$ paléole externe de la glycérie flottante; $d$ coupe de la paléole externe; e portion de feuille avec sa ligule membrancuse.

11. 


\section{ANALYSE DES ESPE்CES.}

1) Paléole externe à $7-9$ nervures. .

1 Paléole externe à 4-5 nervures. . .

f Panicule régulière ample. . . . . Glycérie AQUati-

2 Panicule longue, effilée, unilatérale QUE (a). ou presque unilatérale . . . . . G Grycérie flottante

Feuilles planes; rameaux de l'étage (b). inférieur de la panicule disposés par 2-7; paléole externe obtuse, subironquée.................. Glycérie distante

5 Feuilles plus ou moins roulées en gouttière, ou pliées; étage inférieur de la panicule à 2-3rameaux; paléole ex- GLycÉrie maritume terne un peu obtuse. . . . . . . . (d).

a) Glycérie élevée. (Glyceria spectabilis $\mathbf{M}$ et K.) Souche rampante, genouillée; chaumes de 10 à 18 décimètres, droits, forts, plus ou moins comprimés ou cylindriques, striés, glabres ; feuilles longues, larges, rudes et coupantes sur les bords, marquées d'une tache triangulaire brunàtre ou fauve à l'entrée de la gaîne qui est entière ou presque entière; ligule courte, arrondie ou presque tronquée, présentant le plus souvent une pointe plus ou moins allongée vers son milieu; panicule régulière, ample, étalée, très-rameuse; locustes à $4-9$ fleurons verdàtres ou un peu rougeâtres; paillettes à une nervure; paléole externe obtuse, à sept nervures bien. prononcées; locustes quelquefois vivipares. Vivace; fleurit de juin à juillet.

b) Glycérie flottantc.(Glyceria fluitans.R. Br.) Souche rampante; chaume de 5 à 9 décimètres, assez gros, mais peu consistant, flasque, plus ou moins droit ou ascendant, strié, un peu comprimé, glabre, recouvert souvent jusqu'a la panicule par les gaines des feuilles; feuilles assez larges, plus ou moins carénées, molles, un peu rudes sur les bords 
et sur le dos; ligule allongée; panicule longue, effilée, à rameaux d'abord dressés, s'écartant ensuite à angle droit pendant la floraison, unilatérale ou presque unilatérale; locustes grêles, lisses, cylindriques, atteignant ordinairement deux centimètres de longueur et contenant 7-12 fleurons d'un vert tendre; paléole externe à sept nervures, blanchâtre et argentée en ses bords.

Il y en a deux variétés bien distinctes : l'une à paléole externe, à peine dentée ou rongée, et à rameaux de la panicule ternés ou quaternés inférieurement, le plus long très-ramifié, portant plus de quatre locustes, les deux plus cour's n'en portant qu'une ; l'autre à paléole externe marquée ordinairement de sept dents colorées et à rameaux inférieurs de la panicule le plus souvent ternés, le plus long ne portant jamais plus de trois locustes, les autres n'en portant qu'une. Vivace; fleurit en mai-juin.

Bords des fossés, prairies humides et tourbeuses.

c) Glycérie distante. (Glyceria distans Waht.) Souche cespiteuse; chaume de $\mathbf{2}$ à $\mathbf{3}$ décimètres, couché à la base ou presque droit, nu supérieurement; feuilles étroites, de 5 à 6 millimètres de largeur, planes ou un peu roulées en dessous; panicule égale, régulière, diffuse, à rameaux longs disposés par 4-8, divariqués ou réfléchis après la fécondation, rarement resserrés; locustes à 4-7 fleurons; paillettes très-obtuses, l'une uninerviée et l'autre trinerviée; paléole externe à 4-ว̆ nervures, comme tronquée et denticulée au sommet, l'intérieure émarginée, à peine ciliée. Vivace; fleurit de juin à août.

Mares et fossés salins.

d) Glycérie maritime. (Glyceria marilima 
Pauq.) Souche cespiteuse(1), émettant souvent des rejets stériles quelquefois plus long̀s que les fertiles, paraissant être formés par une série de gaines qui se dégagent les unes des autres; chaumes de $\overline{3}$ à 9 décimètres, couchés et coudés à leurs nœuds; feuilles radicales nombreuses; limbes linéaires plus ou moins roulés, jamais planes; panicule régulière ou unilatérale, à rameaux disposés par 2-5, plus ou moins resserrés après la fécondation ; locustes linéaires à 4 -12 fleurons; paillettes marquées chacune de 5 nervures; paléole externe à $\zeta$ nervures, obtuse et membraneuse au sommet.

Il en existe deux variétés: l'une à panicule étalée lors de la fécondation et resserrée avant et après, et l'autre à panicule contractée subspiciforme. Vivace; fleurit en juin.

Sables maritimes.

GENRE 16. - SCLÉROCHLOE (SCLEROCHLOA P. B. B.)

Le genre sclérochloë ne diffère essentiellement dı genre glycérie que par sa paléole externe émarginée-mucronée.

\section{ANALYSE DES ESPÈCES.}

\section{Locustes ovales-oblongues à $4-5$} feurons; rameaux portant la plupart de 3 à 15 locustes. . . . . . . . Screrochloè cou-

Locustes linéaires-lancéolées à CHéE (a). 6-13 fleurons; rameaux la plupart ne Sci.erocriloe rodde portant qu'une locuste...... (b).

(1) Beaucoup de botanistes croient que la souche de la glycéric mavilime émel des rhizomes tracants; mais rien n'est moins fondé que refte assertion ; ear elle ne produit que des rejets siériles décombants qui émettent des racines quand leur's nieuds sont enterrés. Or, e'esi ce qui arrive à cette espèce dans les sables maritimes où le reflux àmène du sable qui opère ainsi une espèce de marcottagr 
DEUXIĖME PARTIE. - ANALYSES ET DESCRIPTIONS. 135
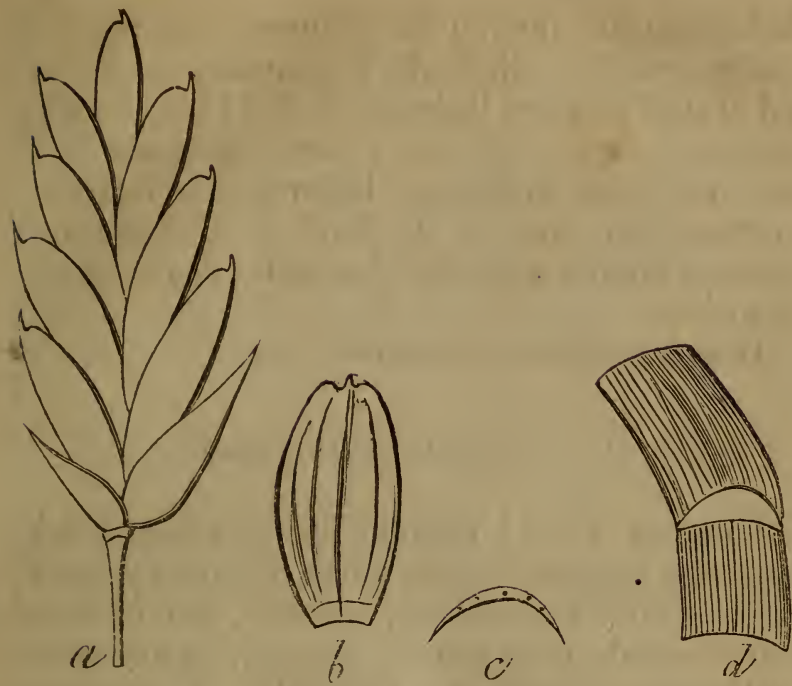

Fig. 19 (1).

a) Sclerochloë couchée. (Sclerochloa procumbens P. D. B.) Souche cespiteuse; chaumes de 2 à 7 décimètres, couchés, redressés; feuilles planes; ligule courte ; panicule roide, unilatérale, à rameaux cylindriques, rudes; locustes ovales oblongues, à 3-丂 fleurons; paléole externe obtuse, presque tron-

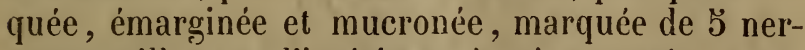
vures saillantes; l'intérieure à peine rongée ou entière. Annuelle; fleurit en août et septembre.

Dans les sables maritimes; très-rare en Belgique.

b) Sclerochloë roide. (Sclerochloa rigida L. K.) Souche cespiteuse; chaumes de 1 à 5 décimètres, coudés, diffus; feuilles étroites, planes ou roulées,

11) $a$ Locuste de slerochloë roide, grossie; $b$ paléole externe de sclerochloë couchée; $c$ coupe de la méme; $d$ portion de feuille avec sa ligule membraneuse courte. 
de la longueur environ du chaume; panicule resserrée, roide, unilatérale, à rameaux la plupart à $1-2$ ou 5 locustes linéaires de 5 -1 1 fleurons plus

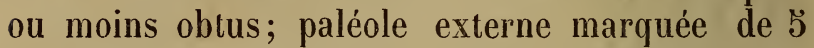
nervures assez saillantes, légèrement échancrée, avec un petit mucron au fond de l'échancrure, l'interne bifide ou dentée. Annuelle; fleurit en mai et en juin.

Dans les sables et les endroits secs.

\section{genre 17. - aelbroeckie (aelbroeckia N.)}

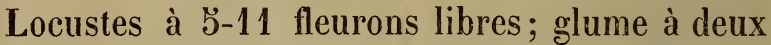
paillettes inégales, aiguës, concaves, non carénées ; paléole externe mucronée, concave, non carénée, dépourvue de poils courts, laineux, ou aranéeux; l'intérieure mucronée; paléolules 2, membraneuses, entières ou bidentées, ou acuminées, étamines 3 ; ovaire glabre; styles très-courts; stigmates 2, plumeux; grain oblong; locustes dispo-
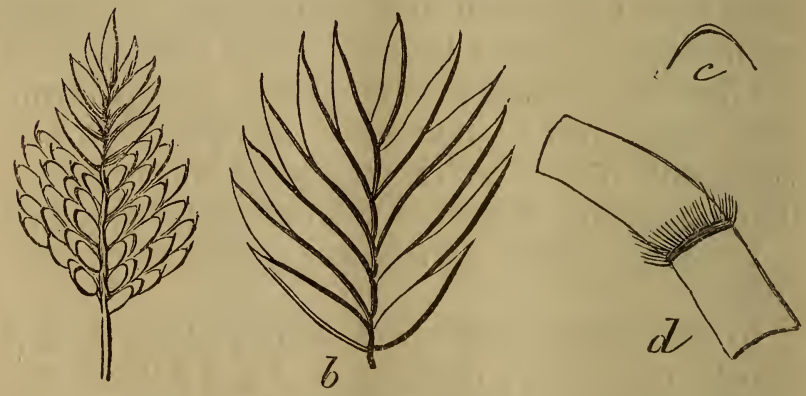

Fig. 20 (1.)

(1) a Division secondaire de la paniculespiciforme ou épi composé, grossie; $b$ locuste grossie; $c$ coupe de la paléole externe; $d$ portion de feuille avec sa ligule inembraneuse courte, fortement cilice. 
sées en épi composé unilatéral; axe primaire de l'inflorescence se divisant en axe secondaire portant des locustes sessiles ou subsessiles; ligule fortement ciliée.

Aclbroeckie maritime. (Aelbrocckia maritima Nob.) Chaume de 5 à 5 décimètres, roide, glauque; feuilles distiques, glauques, roides, roulées ou pliées en gouttière; ligule membraneuse, mais fortement ciliée, ressemblant d'abord à une rangée de poils; épi composé, interrompu, unilatéral ; locustes serrées, renfermant chacune ל̌-10 fleurons distiques, glabres, d'un vert un peu rougeàtre, devenant assez fréquemment violet ì la maturité. Vivace; fleurit en juillet.

Sables maritimes de la Flandre occidentale.Rare.

\section{III sous-tribu. - Poacées.}

Tous les fleurons dépourvus d'un involucelle de longs poils soyeux; pas de locustes réduites à des bractées pectinées ou pinnées; paléole externe carénée.

$$
\text { GenRe 18. - DACTyle (DAGTylis L.) }
$$

Locustes à 2-8 fleurons, fortement comprimées et courbées-concaves, rapprochées en glumerules compactes; paillettes inégales, lancéolées et carénées, à côtés inégaux, leplus large convexe, leplus étrcitconcave; paléole extérieure carénée, à cinq nervures portant une arête courte, terminale ou presque terminale, l'intérieure bicarénée, bidentée au sommet; paléolules 2, lobées, inégales; ovaire turbiné, glabre; styles raccourcis, terminaux; stigmates 2 , plumeux; grain allongé, se détachant souvent de la 
glumelle par le battage; panicule unilatérale, lâche, lobée ou spiciforme.
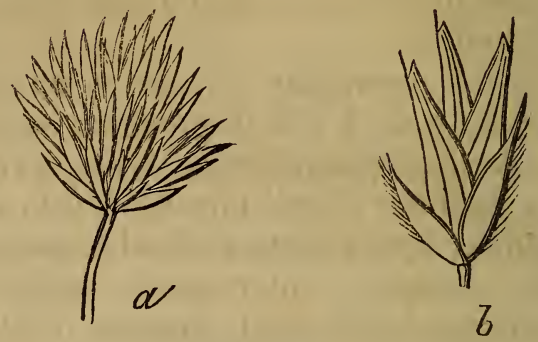

Fig. 21 (1).

Dactyle gloméré. (Dactylis glomerata L.) Souche cespiteuse; chaumes de 7 à 12 décimètres, forts, droits ou un peu coudés à la base; feuilles carénées ou planes, pliées dans leur jeunesse, plus ou moins rudes, ainsi que leurs gaînes, qui sont comprimées ; liguleallongée, déchirée; panicule formée de plusieurs pelotons de fleurs serrées, la plupart tournés du même côté, le peloton inférieur porté par un pédoncule assez long; panicule étalée à la floraison et resserrée avant et après. La panicule de cette plante est plus ou moins fournie et les rameaux sont droits ou étalés. Vivace; fleurit en juin et en juillet.

Dans les prés, le long des chemins el des haies.

\section{genRe 19. - paturin (}

Locustes comprimées latéralement, à 2-50 fleurons; scobine se détachant avec les fleurons en autant d'articles; pailletles plus ou moins herbacées, entières; paléole externe carénée, entière ou émargince, mutique, à $1-5$ nervures plus

(1) a Glomerule de locustes du dactyle gloméré ; $b$ locuste du mème. 
ou moins marquées, glabre, velue ou munie dans sa partie inférieure de poils laineux ou aranéeux; paléole intérieure bicarénée, entière, émarginée, bidentée ou tronquée; paléolules 2, entières, obtuses, tronquées, ou présentant une dent latérale; ovaire glabre, presque rond; styles courts, terminaux; stigmates 2, plumeux; grain oblong, oblongtrigone ou subglobuleux, étroitement renfermé entre les paléoles, présentant un sillon sur une de ses faces; panicule rameuse, à locustes non rapprochées en glomerules; ligule membraneuse.

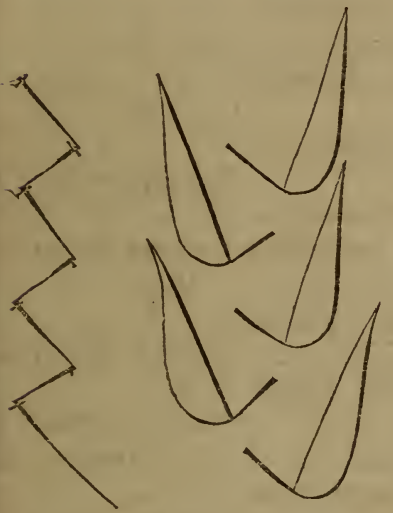

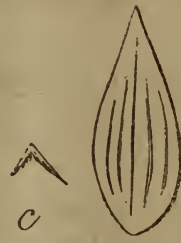

Fig. 22 (1).

ANALYSE DES ESPÈCES.

Souche émettant des rhizomes lon-

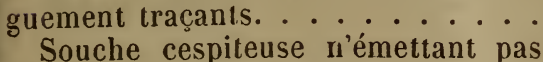
des rhizomes traçants. . . . . . .

Feuilles ayant à peine 3 millimètres de largeur. . . . . . . . . . .

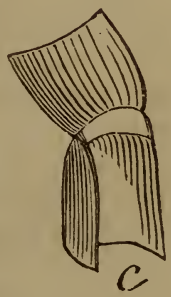

Feuilles ayant environ le double de Paturiv de Silésie largeur. ............ (j).

(1) $a$ Scobine entière; $b$ fleurons munis chacun d'une portion de la scobine; $c$ coupe de la paléole externe; $d$ paléole externe; $e$ portion de feuille munie de sa ligule membraneuse. 
(Chaume fortement comprimé,à deux angles tranchants; locustes à 5-9 fleu- Paturin comprimé ${ }_{3}$ rons; panicule presque unilatérale . . Chaume presque cylindrique, rarerement comprimé, à כ̆-5̆fleurons; pani- Paturin des PRÉs cule égale. ............

Chaume renflé en bulbe à la 4 base; locustes souvent vivipares. . . Paturin bulbeux(c) Chaume non renflé à la base. . . . 5 .

Gaîne de la feuille supérieure plus ô longue que le limbe. ........

o Gaîne de la feuille plus courte que le limbe. . . . . . . . . . . .

Rameaux de la panicule géminés ou ${ }_{6}\left\{\begin{array}{c}\text { solitaires, très-rarement ternés. } \\ \text { Rameaux de la panicule disposés } \\ \text { par verticille de } 3-7 . \ldots . . . .\end{array}\right.$

(h). (i).

Paléole externe glabre, subéchan$7\left\{\begin{array}{l}\text { crée, mucronée au sommet. . . . . . . Paturin annuel (a) } \\ \text { Paléole externe pubescente ou ve- }\end{array}\right.$ lue à la base, sur le dos et sur les Paturin des alpes borls. .............. (b).

Ligule courte, tronquée; plante 8 d'un glauque bleuâtre. ....... Paturin bleuatre 8 Ligule oblongue-lancéolée, plante (g).

verte. .............. Paturin commun $(\mathrm{f})$ Ligule courte, presque nulle . . . Paturin des bois (d) 9 Isigule de la feuille supérieure oblongue aiguë. . . . . . . . Paturin fertile (e)

A. - Souche cespiteuse n'émettant pas des RHIZOMES LONGUEMENT TRAC̣ANTS.

a) Paturin annuel. (Poa annua L.) Souche cespiteuse ; chaumes de 2 à ら̌ décimètres, ordinairement obliques, comprimés au-dessus du deuxième noud inférieur, coudés, quelquefois radicants, non en bulbes à la base, feuillés du bas; feuilles planes ou ondulées sur leurs bords; ligule des feuilles su- 
périeures oblongue; panicule le plus souvent unilatérale ou hémicycloïde; rameaux inférieurs géminés ou solitaires, très-rarement ternés, trèslongs relativement aux supérieurs, s'ouvrant presque toujours à angle droit avec l'axe primaire de l'inflorescence, ou se réfléchissant en quelque sorte sur le chaume; locustes ovales-oblongues à 5-7 fleurons verdàtres ou rougeâtres; paléole externe souvent émarginée ou échancrée, glabre. Annuel; fleurit toute l'année.

Très-commun partout.

b) Paturin des Alpes. (Poa alpina P. C.)

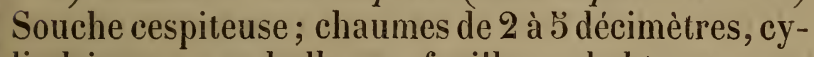
lindriques, non bulbeux; feuilles subobtuses, mucronées et courbées en gouttière au sommet, à gaîne plus longue que le limbe de la feuille supérieure; ligule des feuilles inférieures courte, tronquée, celle des feuilles supérieures quelquefois oblongue-lancéolée, aiguë; panicule régulière ou unilatérale, plus ou moins étalée; rameaux inférieurs géminés; locustes ovoïdes à 3-7 fleurons libres, pubescentes à la base, sur le dos et sur les bords, rarement liées par des poils aranéeux; paillettes et paléoles externes soyeuses ou hérissées, parfois un peu recourbées en crochet; locustes assez souvent vivipares. Vivace; fleurit en juin et en juillet.

Terrains secs, sables maritimes.

c) Paturin bulbeux. (Poa bulbosa L.) Souche cespiteuse; chaume de 4 à 7 décimètres, droit, glabre, presque nu; feuilles glabres, planes, étroites, les supérieures courtes, à gaînes très-longues; les inférieures nombreuses, ramassées en gazon; ligule oblongue plus ou moins aiguë ; gainnes jaunâtres formant une espèce de bulbe à la' base; panicule égale ou un peu unilatérale, souvent un peu 
contractée à la maturité, flexueuse au sommet; rameaux inférieurs géminés ou verticillés, scabriuscules; locustes ovales à 3-7 fleurons; paléole externe n'ayant ordinairement qu'une nervure, velue sur le dos et sur les bords, l'intérieure bifide.

Il y a une variété à locustes vivipares; paléoles quelquefois allongées et développées en forme de feuilles ; panicule paraissant comme chevelue et frisée; feuilles inférieures jaunàtres. Vivace; fleurit en mai et en juin.

d) Paturin des bois. (Poa nemoralis L.) Souche cespiteuse ou presque traçante; chaume de 4 à 9 décimètres, courbé ou droit au sommet, non en bulbe à la base; feuilles supérieures à gaîne plus courte que le limbe; ligule courte, presque nulle, à peine distincte; panicule droite ou penchée; rameaux inférieurs presque toujours disposés par 3-5, ordinairement dressés; locustes à 2-3 fleurons

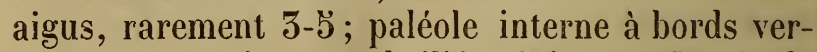
dâtres ou scarieux, subciliée. Vivace; fleurit de mai à juillet.

Croît dans les bois, les lieux ombragés, les haies, les fentes des rochers, les sols rocailleux.

Le paturin des bois présente un assez grand nombre de variétés, qui sont :

$1^{\circ}$ Le paturin noueux. Panicule régulière ; chaumes émeitant au-dessus des nouds des fibres radicales adventives formant des paquets oblongs ou subglobuleux; feuilles et gaines vertes.

$2^{\circ}$ Le paturin des bois subuniflore. Panicule simple, â rameaux solitaires ou géminés, ou presque simple, ne portant qu'une dizaine de locustes vertes environ; feuilles et gaines vertes.

$3^{\circ}$ Le paturin des bois tenelle. Panicule droite à rameaux disposés par 4-6, étalés, portant la 
DEUXIĖME PARTIE. - ANALYSES ET DESCRIPTIONS. 141

plupart, même les inférieurs et les plus longs, 1 à 4 locustes vertes; feuilles et gaines vertes.

$4^{\circ}$ Le paturin des bois vulgaire. Panicule large, assez ample; rameaux inférieurs disposés par 4-7, presque étalés, les plus longs portant $\breve{a}$ à 15 locustes vertes; feuilles et gaines vertcs.

$5^{\circ}$ Le paturin des bois des montagnes. Panicule resserrée, à rameaux inférieurs disposés par 2-6, les plus longs portant 4-7 locustes rougeàtres ou violacées; feuilles et gaines vertes.

$6^{\circ}$ Le paturin des bois resserré. Panicule allongée, resserrée, à rameaux disposés par 5-7, les plus longs portant 4 à 10 locustes teintes de jaune; feuilles et gaines vertes.

$7^{\circ}$ Le paturin des bois ferme. Panicule ferme, resserrée, à rameaux disposés par 5-6, les inférieurs plus longs, presque appliqués contre l'axe portant $\breve{\jmath}$ à 7 locustes vertes; feuilles et gaînes verles.

$8^{\circ}$ Le paturin des bois glaucescent. Panicule à rameaux plus ou moins étalés, les plus longs portant 5 à 10 locustes; feuilles et gaines d'un vert foncé glauque : c'est le paturin glauque de Smith.

e) Paturin fertile (Poa fertilis Host.) Souche cespiteuse rarement presque traçante; chaumes de 4 à 9 décimètres, très-souvent radicants à la base ; feuilles glabres, étroites, pointues, ordinairement lisses, ainsi que les gaines, qui sont plus courtes que le limbe; ligule des feuilles supérieures oblongue, aiguë; panicule égale, à rameaux disposés par 4-10 locustes ovales-lancéolées à 2-5̆ fleurons légèrement velus sur le dos et à a base; scobine en massue saillante au sommet, parfois in peu aranéeuse sous la massue.

Le paturin fertile de Host ne parait être qu'une 
variété du paturin à feuilles étroites de $\mathbf{L}$. (Poa angustifolia); il en est de même du paturin épars (Poa effusa), et du paturin des marais (Poa palustris ) de Roth. Quoi qu'il en soit, nous regardons le paturin fertile de Host comme la souche des deux autres qui n'en sont que des variétés. Le paturin fertile des marais présente la panicule la plus fournie des trois, ensuite le paturin fertile vulgaire, et enfin le paturin épars, qui a la panicule la moins fournie. Vivace; fleurit de mai à septembre.

Dans les prairies, au bord des rivières et des fossés.

f) Paturin commun. (Poa trivialis L.) Souche cespiteuse; chaume de 5 à 15 décimètres, glabre, cylindrique, rude, quelquefois lisse au-dessous de la panicule; feuilles glabres, planes, pointues; gaîne rude, plus longue que le limbe; ligule allongée, aiguë; panicule ferme, à rameaux inférieurs au moins ternés, le plus souvent disposés par 4-7, formant souvent presqu'un angle droit avec l'axe : quelques rameaux avortent parfois et se présentent alors sous la forme d'appendices blanchâtres; locustes à $\mathbf{3}$-ə̆ fleurons laineux à la base; paléole externe à cinq nervures; scobine subsétiforme, saillante au sommet.

Le paturin commun présente quelques variétés locales qui se distinguent par leur panicule plus ou moins fournie et étalée. Vivace; fleurit de mai à juillet.

Dans les prairies, les fossés, sur les bords des chemins et des champs.

g) Paturin bleuâtre. (Poa coesia Dmtr.) Souche cespiteuse; chaume de 4 à 7 décimètres, trèsglauque, bleuâtre, ainsi que les feuilles; limbe plus 
court que la gaine; ligule courte, tronquée ou subtronquée; panicule à rameaux robustes, étalés, les inférieurs disposés par 3-9, formant un angle presque droit avec l'axe de l'inflorescence, rarement dressés à l'époque de la floraison, plus ou moins resserrés avant et après; locustes à $5-7$ tleurons libres, rarement liés entre eux par des poils aranéeux; paléole externe pubescente sur la carène et sur les bords ; paléole interne à peine émarginée, ciliée, pubescente. Vivace; fleurit en mai et en juin.

Rochers et collines des environs de Spa.

B. - SOUChe ÉMETTANT DES RHIZOMES LONGUEMENT TRAĢANTS.

h) Paturin des prés. (Poa pratensis L.) Souche émettant des rhizomes longuement traçants; chaume de 4 à 12 décimètres, cylindrique ou ancipité, lisse ou à peine scabriuscule; feuilles très-variables; gaîne supérieure plus longue que la limbe; ligule courte, tronquée ou très-obtuse; panicule étalée, égale, diffuse ou oblongue, un peu resserrée; rameaux inférieurs disposés par 5-7; locustes ovales, verdàtres ou colorées, à 5-5 fleurons laineux à la base.

Cette plante présente beaucoup de variétés locales, dont les principales sont :

Le paturin des prés vulgaire. Chaume cylindrique, de 3 à 9 décimètres; feuilles radicales et caulinaires de même largeur; plante verte.

Le paturinà larges feuilles. Chaume cylindrique, de 2 à 5 décimètres; feuilles larges; plante verte.

Le paturin de Lejeune. Chaume cylindrique, de 
3 à 4 décimètres; panicule ferme, presque roide; locustes colorées; plante verte.

Le paturin glauque. Chaume cylindrique; plante entièrement glauque, lisse.

Le paturin à feuilles étroites. Chaume cylindrique, de 5 à 12 décimètres, presque nu; feuilles très-étroites, pliées, presque sétacées.

Le paturin ancipité. Chaume ancipité.

Le paturin élancé. Chaume cylindrique, de 4 à 12 décimètres; feuilles étroites, presque enroulées, glaucescentes, rudes.

i) Paturin comprimé. (Poa compressa L.) Souche longuement traçante; chaume de 3 à 6 décimètres, fortement comprimé, à deux angles tranchants, glabres, lisses, coudés, à demi couchés; feuilles glabres, planes, courtes, étroites et pointues; gaîne plus longue que le limbe; ligule très-courte, très-obtuse ou tronquée; panicule plus ou moins roide, serrée, comprimée, unilatérale, à rameaux supérieurs presque de même longueur apparente que les inférieurs; locustes ovoïdes-oblongues, à fleurons, à peine pubescents à la base, souventrougeâtres sur les bords. Vivace; fleurit de juin à août.

Terrains secs et sablonneux, parmi les décombres et sur les vieux murs.

j) Paturin de Silésie. (Poa sedutica Haenke.) Souche longuement traçante; chaume de 4 à 13 décimètres, droit, scabre, aplati, strié, un peu genouillé dans le bas; feuilles glabres, larges, pointues, planes à la base, plus ou moins pliées au sommet, rudes sur les bords ; gaine comprimée en forme de carène, plus longue que le limbe; ligule très-courte; panicule oblongue, ample, étalée, à rameaux scabres, la plupart disposés par cinq; locustes oblongues, ovales, glabres, ordinairement at 
trois fleurons; paléole externe nerviéc, ne présentant point de poils à la base.

Il en est une variété qui est verte dans toutes ses parties et une autre qui est rougeâtre ou violette. Vivace; fleurit en juin.

Bois montagneux.

Genre 20. - Gatabrose. (Catabrosa P. D. B.)

Locustes comprimées latéralement, à 2-5 fleurons; scobine se détachant avec les fleurons en autant d'articles; pailleltes subherbacées, l'extérieure plus courte, l'intérieure obovale, rongée en ses bords ; paléole externe carénée, trigone, tronquée, denticulée et scarieuse au sommet, à 5-כ̌nervures, l'intérieure convexe, bicarénée, tronquée, émarginée; paléolules 2, libres, un peu tronquées; ovaire glabre; stigmates 2 , plumeux, subsessiles, terminaux, à base persistante ; grain oblong, un peu comprimé; panicule rameuse.

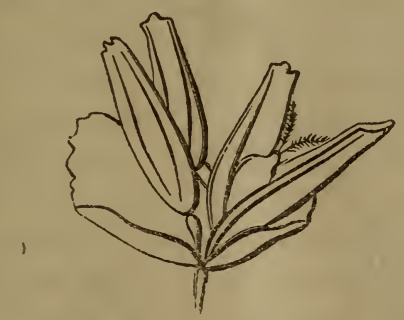

Fig. 25 (1).

Catabrose aquatique. (Catabrosa aquatica $\mathrm{P}$. D. B.) Chaumes de 3 à 18 décimètres, couchés, radicants dans leur partie inférieure, ordinairement nageants, d'où s'élèvent à angle droit, de distance en

(1) Locuste étalée du catabrose aquatique. 
distance, des rameaux de 3 à 7 décimètres, tendres, lisses, glabres ; feuilles planes, lisses, glabres, courtes; ligule øblongue, entière ou déchirée; panicule lâche, plus ou moins étalée; locustes presque toujours biflores, rarement 3 -כ̆ fleurons souvent violacés; paléole externe glabre ou velue, verdâtre ou rougeâtre; l'une des paillettes de la longueur environ de la scohine qui supporte le deuxième fleuron. Vivace; fleurit de mai à juillet.

Dans les prairies humides à sol sablonneux, les fossés, les mares.

genre 21. - Éragrostide. (eragrostis P. D. B.)

Locustes comprimées latéralement, linéaires-
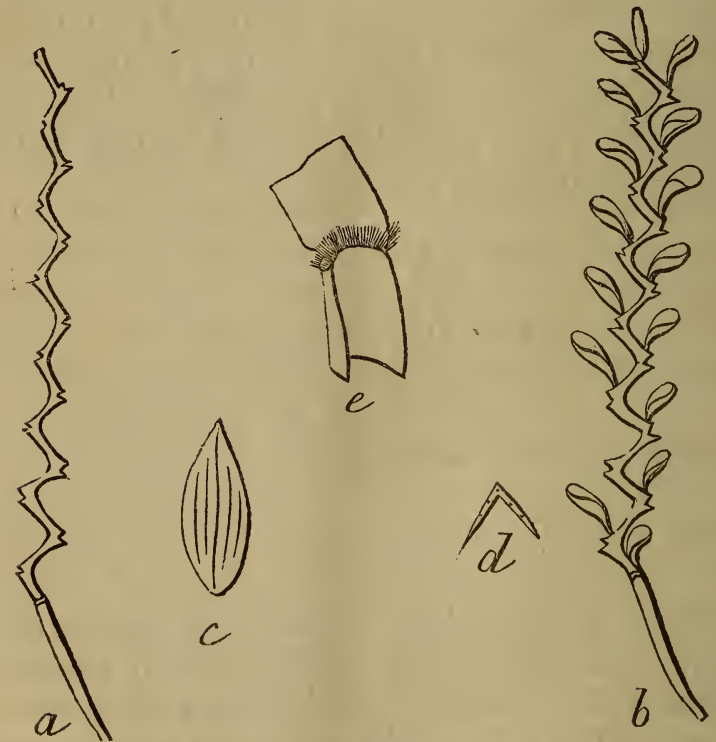

Fig. 24 (1).

(1) $a$ Scohine persistante de l'éragrostide vulgaire ; $b$ la même portant la paléole interne; $c$ paléole externe; $d$ coupe de la paléole externe; $e$ portion de feuille avec sa ligule pileuse. 
oblongues, presque planes, contenant 5 à 50 fleurons, à scobine persistante; paillettes membraneuses mutiques, caduques, inégales; paléoles membraneuses mutiques, l'extérieure caduque, subherbacée, obtuse, entière ou émarginée, embrassant l'intérieure qui est bicarénée, persistante sur le rachis; paléolules 2 , peu charnues, entières, obtuses ou tronquées, plus courtes que l'ovaire; ovaire glabre; styles allongés, terminaux; stigmates 2, plumeux; grain transparent, subglobuleux, à peine comprimé ; panicule rameuse.

\section{ANALYSE DES ESPĖCES.}

Rameaux de la panicule solitaires Éragostride vuLou géminés.

GaIRE (b)

Rameaux de la panicule disposés Éragostride poilue par verticille de $4-6 . \ldots \ldots \ldots$

a)Eragrostide poilue. (Eragrostispilosa P.D.B.) Racines fibreuses, glabres; chaumes nombreux, de 1 à 4 décimètres, dressés ou obliques; feuilles d'abord planes, puis roulées au sommet, ordinairement d'un vert pâle; ligule pileuse; gaînes présentant deux faisceaux de poils sur le niveau et en dehors de la ligule; panicule à verticilles inférieurs formés de 5- rameaux, égale, diffuse, allongée, peu étalée pendant la floraison, ouverte à la maturité ; locustes linéaires à 5̆-12 fleurons; paléole extérieure à trois nervures, les latérales peu marquées ou nulles. Annuelle; fleurit en juin et en juillet.

Bords de la Meuse. (?)

b) Éragrostide vulgaire. (Eragrostis vulgaris Coss. et Germ.) Racines fibreuses, parfois pubescentes; chaumes nombreux, rameux, obliques; feuilles larges, ordinairement d'un vert foncé, glabres ou parsemées de poils rares, ainsi que la 
gaine; ligule pileuse, rarement deux faisceaux de poils sur le niveau et en dehors de la ligule; panicule à rameaux solitaires, régulière, d'un vert noirâtre et presque roussâtre à la maturité; locustes lancéolées à 8-20 fleurons distiques; paléole externe marquée de trois nervures dont les deux latérales très-saillantes. (?)

MM. Cosson et Germain qui ont fondé l'Éragrostide vulgaire y rapportent l'Eragrostide grande (Eragrostis major) de Host ou la Brize éragrostide de Linné et l'Eragrostide faux paturin de Palisot de Beauvois. Nous croyons que ces habiles botanographes ont eu raison en agissant ainsi, les caractères qui avaient servi de bases n’étant tirés que du plus ou moins de développement d'un organe qui n'a pas l'importance que certains agrostographes lui attribuent.

\section{IWe sous-tribu. - Arundinées.}

Locustes à 1-6 fleurons, l'inférieure mâle, glabre, les autres pourvues à la base de longs poils soyeux; paléole extérieure carénée; panicule trèsrameuse.

GENRE 22. - ROSEAU. (ARUNDO L.)

Locustes comprimées latéralement, à 2-6 fleurons, l'inférieure mâle dépourvue de poils à la base, les autres entourées chacune de longs poils soyeux; paillettes carénées, aiguës, plus courtes que la locuste, inégales; l'inférieure de moitié plus petite que l'intérieure; paléole extérieure étroite, allongée, subulée, presque deux fois plus longue que l'intérieure qui est bifide, dentée; paléolules tron- 
quées, presque frangées. Ovaire glabre; styles 2, terminaux, assez longs; stigmates en goupillon, sortant vers la partie moyenne du fleuron; grain oblong non sillonné; panicule très-rameuse, brunâtre, roussâtre ou violacée.

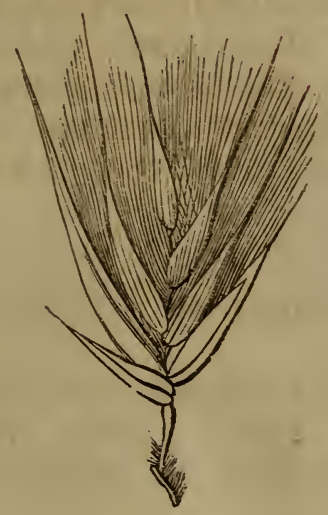

Fig. 23 (1).

Roseau à balais. (Arundo phragmites L.) Souche émettant des rhizomes longuement traçants; chaumes de 1 à 2 mètres, fermes, droits, trèsfeuillés; feuilles assez longues, larges, planes, glabres, glaucescentes, rudes ct coupantes en leurs bords, finissant en une longue pointe ; ligule cotonneuse ou pileuse; panicule très-rameuse; locustes membraneuses, minces, violettes ou d'un jaune fauve, à 1-6 fleurons ; paléole externe très-longue, étroite, pointue.

Cette plante offre deux variétés qu'on a considérées comme des espèces. La première, qui est l'espèce type de Linné, présente des locustes la

(I) Locuste de roscau à balais. 
plupart fertiles, à $\breve{5}-\check{\text { fleurons; }}$ la seconde a une panicule moins ample et des locustes la plupart stériles, neutres ou mâles, les locustes fertiles ordinairement à un fleuron hermaphrodite, la plupart placées sur la partie supérieure de la panicule. C'est le roseau noirâtre de Merat (A rundonigricans Merat). Vivace ; fleurit d'août à octobre.

Ce géant des graminées de notre pays est trèsabondant dans les fossés aquatiques, sur les bords des étangs, des rivières et dans les prairies humides.

ve sous-tribu - Tiodiées.

Paléole externe arrondie, non carénée; locustes embrassés en grande partie par la glume, à 2-6 fleurons dont les supérieurs au moins stériles.

GENRE 25. - MÉlique. (Melica L.)

Locustes à 2-4 fleurons dont un ou deux supé rieurs stériles rudimentaires; paillettes convexes, mutiques, presque égales; paléoles mutiques, presque égales, coriaces, l'extérieure semi-cylindrique; une ou deux paléolules, libres ou soudées entre elles ; ovaire ovoïde, turbiné, glabre; styles courts, terminaux ; stigmates 2, plumeux; grain ovoïde, oblong, comprimé, non sillonné; panicule spiciforme ou lâche, unilatérale ou presque unilatérale ; chaume présentant plusieurs nouds placés et espacés normalement. 

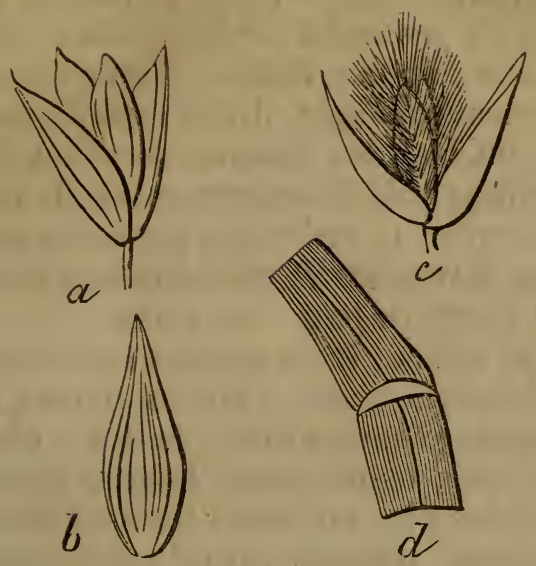

Fig. 26 (1).

ANALYSE DES ESPÈCES.

1

Paléole externe glabre. ....

Paléole externe velue-ciliée. . . Méliq ue cilíé (a).

Chaumes forts, dressés d'un mètreet plus; feuilles larges; panicule spici-

2 forme, unilatérale, très-fournie. . . MÉLIQUe ÉLEvÉE(d).

Plantes ne réunissant pas ces caractères. . . . . . . . .

3.

Gaîne des feuilles présentant à Mélique uniflorli llopposite du limbe un appendice vert.

Pas d'appendice vert à l'opposite du Mélique pexchéc limbe.

(1) Mélique ciliée. (Melica ciliata L.) Souche cespiteuse; chaumes de 4 à 9 décimètres, nom-

(1) a Locuste de mélique penchée; $\iota$ paléole externe de la mème; c locuste de mélique ciliée; $d$ portion de feuille munie de sa ligule membraneuse. 
breux, droits, glabres, grêles, roides, d'un vert glauque, un peu rudes; feuilles rudes, pubescentes, étroites, un peu roulées ; ligule oblongue, bifide; panicule allongée, droite, spiciforme, le plus souvent unilatérale; locustes à un ou deux fleurons fertiles; paléole externe garnie de longs poils blancs, soyeux et redressés, parallèles pendant la floraison, divergents et très-visibles à la maturité. Vivace; fleurit en juin et en juillet.

Sur les collines et les rochers calcaires.

b) Mélique penchée. (Melica nutans L.) Souche traçante; chaume droit, de 3 à 7 décimètres, glabre, grêle; feuilles assez larges, planes, pointues, à gaîne plus ou moins triangulaire, entière; ligule courte, tronquée; pas d'appendice vert opposé au limbe des feuilles; locustes pendantes, disposées en panicule subspiciforme, unilatérale, penchées; deux fleurons fertiles; paléole externe à 7 nervures, glabre; glume violacée ou rougeâtre. Vivace; fleurit en mai et en juin.

Dans les bois secs et élevés.

c) Mélique uniflore. (Melica uniflora Retz.) Souche traçante; chaume de 4 à 7 décimètres, droit, glabre, grêle, obscurément triangulaire ou tétragone; feuilles glabres, assez larges, pointues, à gaînes triangulaires, entières ; un pelit appendice linéaire vert placé à l'opposite du limbe de la feuille, munie d'une ligule courte; panicule rameuse, ouverte, unilatérale; locustes le plus souvent rougeâtres, droites, à un fleuron fertile; paléole extérieure glabre. Vivace; fleurit en mai et en juin.

Dans les bois ombragés du Luxembourg; abonde dans la province de Brabant, depuis Rillaer à Wesemael (par la Bétasie). 
d) Mélique élevée. (Melica altissima L.) Souche vivace; chaume de 8 à 12 décimètres; feuilles larges, rudes; panicule spiciforme, longue, unilatérale; locnstes à trois fleurons, dont deux hermaphrodites et un neutre subclaviforme, violacées ou rougeâtres, rarement vert-jaunâtre; paillettes ovales, minces, scarieuses.

Fleurit de juin à juillet. Vivace. Peu cultivée.

$$
\text { GENRE 24. - TRIODIE. (TRIOdIA P. D. B.) }
$$

Locustes d'abord cylindriques, puis comprimées latéralement, à 5-8 fleurons, dont le supérieur stérile; glume à deux paillettes un peu arrondies, mutiques, presque égales; paléole extéricure tridentée; l'intérieure bicarénée, mutique; paléolules 2 , entières ou émarginées; ovaire turbiné, glabre; styles courts, terminaux; stigmates 2, plumeux; grain ovoïde, comprimé, à peine sillonné-canaliculé, libre ou étroitement enveloppé par les paléoles; panicule simple à 3-8 locustes.
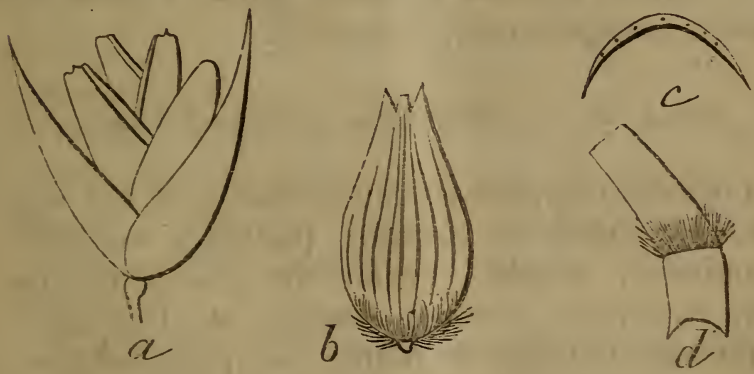

Fig. 27 (1).

(1) $a$ Locuste de triodie inclinée; $b$ paléole externe de la méme: c coupe de la paléole externe; $d$ portion de feuille avec sa ligule pilcuse. 
Triodie inclinée. (Triodia decumbens P. D. B.) Souche cespiteuse ou un peu traçante, émettant un grand nombre de fascicules de feuilles disposées

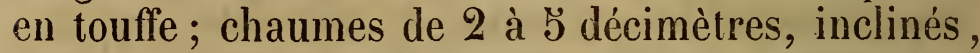
ascendants après la floraison, roides, glabres et lisses ; feuilles dures, planes, garnies sur les deux faces de quelques poils rares, ainsi que sur leurs gaînes, dont l'entrée est munie de deux houppes de poils assez longs; ligule très-courte, pectinée ou pileuse; panicule simple, resserrée, composée de 3-8 locustes, verdâtres ou purpurescentes, solitaires sur les rameaux supérieurs, au nombre de 2-3 sur les inférieurs, contenant 5 -5̆ fleurons, dont il en avorte souvent; paléole externe munie près de ses bords de touffes de poils blancs, satinés. Vivace; fleurit en juin et en juillet.

Dans les bois et les bruyères ou sables humeux incultes.

VIre sous-tribu. - Seslériées.

Locustes à 2-6 fleurons, dont la supérieure ferlile; paléole externe carénée; glume embrassant presque complétement la locuste.

\section{GenRe 25. - KOELERIE. (KoEleria Pers.)}

Locusles comprimées latéralement, à 2-כ̆ fleurons, le supérieur fertile; paillettes carénées, acuminées, inégales, l'extérieure plus petite; paléole extérieure carénée, entière ou légèrement échancrée, mutique ou munie, un peu au-dessous de son sommet, d'une petite arète courte; l'intérieure bicarénée, bifide; étamines 5 ; palénlules inégales, obliquement tronquées ou bi-trifides; ovaire ghabre; styles courts; stigmates, 2, phu- 
DEUXIÈME PARTIL. - ANALYSES ET DESCRIPTIONS. 1\%̈

meux; grain oblong, ni sillonné, ni canaliculé, ni cortiqué par les paléoles; panicule spiciforme, plus ou moins compacte; locustes de la moitié supérieure de la panicule solitaires ou géminées, subsessiles, à pédoncules ne dépassant pas la longueur des locustes.

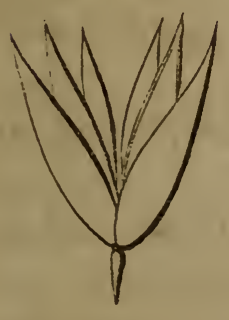

a

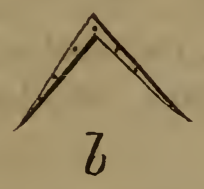

Fig. 28 (1).

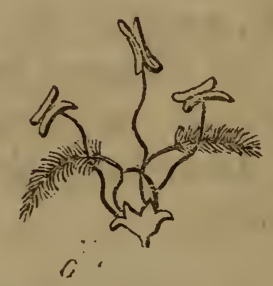

Koelerie crêtée. (Koleria cristata Pers.) Souche cespileuse émettant un grand nombre de fascicules de feuilles formant des touffes serrées; chaume de 2 à 7 décimètres, droit, s'élevant du centre de petites touffes de feuilles; feuilles velues, souvent ciliées; les inférieures étroites, planes ou pliées, pubescentes-ciliées; les caulinaires plus larges, à gaines plus ou moins velues; ligule courte; panicule resserrée en épi souvent interrompu à la base; paléole externe aristée au-dessous du sommet, ou mutique, ciliée sur la carène ou presque glabre.

Cette espèce présente quelques variétés qui sont à :

$1^{\circ}$ Panicule spiciforme non interrompue; locustes inutiques; gaine veloutée; chaume très-grêle ;

(1) a Loru-te de koterie crétéc; $b$ coupe de la paléole externe; c organes sexuels de la mème, présentant en outre àleur base lrs parléolules. 
feuilles étroites, pliées ( $\boldsymbol{K}$. des sables. $\boldsymbol{K}$. arenaria Dmtr.);

$2^{\circ}$ Panicule spiciforme, interrompue à la base; paléole externe, mutique, non ciliée ( $\boldsymbol{K}$. délicate. K. gracilis Pers.);

$3^{\circ}$ Panicule spiciforme, rameuse à la base (K. rameuse. $\boldsymbol{K}$. ramosa);

$4^{\circ}$ Paléole externe munie d'une petite arête (K. aristée. $\boldsymbol{K}$. aristata);

$5^{\circ}$ Chaume très-bas; panicule spiciforme engainée ( $\boldsymbol{K}$. engainée. $\boldsymbol{K}$. vaginata).

Dans les endroits secs, les dunes, et sur les coteaux.

genRe 26. - Seslérie. (SEsleria Ard.)

Locustes comprimées latéralement, à 2-6 fleurons; glume à paillettes inégales, mucronées ou mutiques ; paléole extérieure carénée, irrégulière-

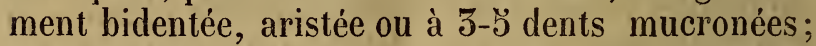
paléole interne bicarénée, tronquée, bifide ou à 4-6 dents; paléolules à 2-5 lobes acuminés, plus longues que l'ovaire; ovaire turbiné, glabre, ou pu-

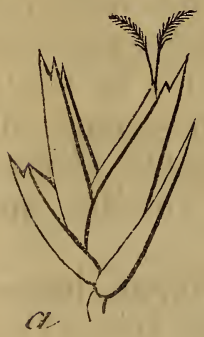

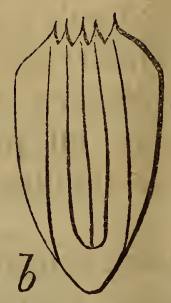

Fig. 29 (1).
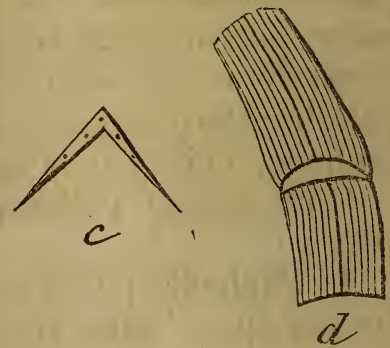

(1) a Locuste de seslérie bleue, grossie; $b$ paléole externe de la nocme; $c$ coupe de la paléole externe; $d$ portion de feuille avec sa ligule membraneuse. 
bescent au sommet; style simple; stigmates trèslongs, pubescents, sortant au sommet des paléoles; grain oblong, un peu comprimé; épi compacte, subglobuleux ou oblong, presque toujours entouré à la base d'un involucre composé de plusieurs bractées caduques, que l'on regarde comme des glumes stériles.

Seslérie bleue. (Sesleria corulea Ard.) Souche cespiteuse traçante, émettant des touffes de feuilles d'où s'élèvent des chaumes droits, grêles, peu feuillés ; feuilles radicales linéaires, planes, dures, roides, arrondies, mucronées au sommet; limbe des feuilles caulinaires court; gaine très-longue, non fendue; épi ovale ou oblong, souvent unilatéral; locustes comprimées à 2-5 fleurons, luisantes, bleuâtres ou violacées; paléole extérieure présentant obscurément 4 à 6 pointes. Vivace; fleurit en avril et en mai.

Sur les collines sèches et calcaires de la Belgique.

IV tribu. - Acéncacées.

Locustes bi ou pluriflores, disposées en une panicule plus ou moins rameuse, rarement spiciforme, compacte ; glume embrassant ordinairement la locuste; paléole externe d'un fleuron au moins, munie d'une arête basilaire, épibasilaire ou dorsale, tordue ou genouillée, rarement avortante par la culture. (Voir figures $30 a b c$, page 15̆8.)

GENRE 27. - CORYYEPHORE. (CORYNEPHORUS P. D. B.)

Locustes comprimées latéralement, à 2 fleurons accompagnés souvent d'un rudiment d'un troisième fleuron; glume à paillettes carénées, mutiques, presque glabres; enveloppant la locuste; paléole 
extérieure entière au sommet, portant vers la base ou au-dessus une arête droite, articuléc à sa partie
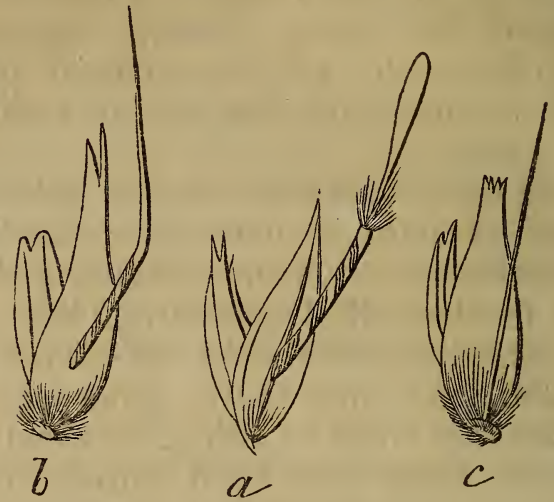

Fig. 30 (1).

moyenne, poilue à l'articulation et renflée en massue au sommet; paléole intérieure bicarénée à la base, trilobée au sommet, imberbe; paléolules 2, presque toujours bifides; ovaire glabre; styles très-courts, subterminaux ; stigmates 2 ; grain oblong, comprimé, enveloppé par les paléoles; panicule rameuse.

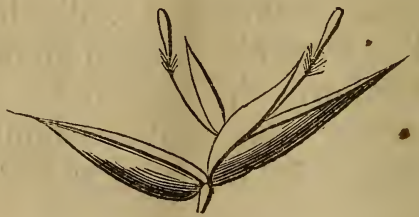

Fig. $\overline{1} 1$ (2).

Corynéphore blanchâtre. (Corynephorus canescens P. D. B.) Plante d'un aspect glauque et

(1) a Fleuron de corynéphore blanchatre, grossi; $b$ fleuron l'avoine caryophillée; $c$ fleuron de eanche cespiteuse.

(2) Locuste de coṛynéphore blanchâtre. 
blanchàtre; chaumes nombreux, disposés en touffes, grêles, lisses, un peu coudés aux nœuds inférieurs; feuilles sétacées, roides et un peu rudes, d'une couleur blanchâtre, celles du haut de la tige ayant une gaine élargie; panicule droite, un peu étalée à la floraison, rarement spiciforme, quelquefois engainée à la base ; locustes biflores, d'une couleur argentée, mêlées de rose et de violet; arête plus courte que la glume.

On trouve parfois, mais très-rarement, un des fleurons de chaque locuste avorté et ne consistant qu'en un simple rudiment. Vivace; fleurit de juin à aoùt.

Se trouve aux endroits sablonneux incultes et stériles.

GENRE 28. - AVOINE. (AVENA L.)

Locustes subcylindriques ou comprimées latéralement, à 2-11 fleurons, le supérieur presque toujours stérile; paillettes convexes plus ou moins comprimées; paléole inférieure bidentée, bipartite ou biaristée au sommet el portant une arète dorsale ou hypodorsale, tordue ou genouillée vers le milieu, rarement droite ou nulle, conséquence de la culture; paléole intérieure bicarénée, mutique; paléolules 2, entières ou inégalement bifides; ovaire glabre ou hérissé au sommet; styles terminaux courts; stigmates 2 ; grain atténué aux deux extrémités, rarement obtus, présentant un sillon sur une de ses faces, glabre ou poilu, étroitement enveloppé par la glumelle, ou se dégageant de celte enveloppe par le battage; panicule étalée, régulière ou unilatérale, rarement spiciforme, subcompacle. 

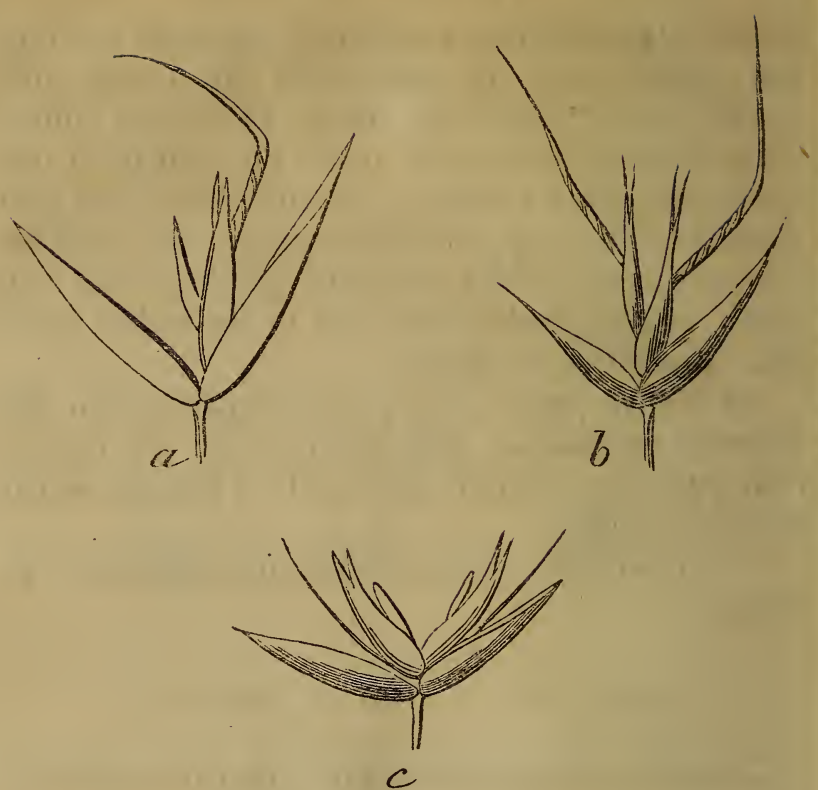

Fig. 32 (1).

ANALYSE DES ESPÈCES.

1

Locustes pendantes. ......

2.

L'ocustes non pendantes.

8.

Paléole externeglabre ou hérissée de

quelques poils rares; scobine glabre.

Paléole externe velue-ciliée, ainsi

que la scobine. ........

3.

7.

I.ocustes à $\overline{3}-8$ fleurons, dépassant

la glume; grain se détachant de sa

3 balle à la maturité. ....... Avorne nue (a).

Locustes à 2-3 fleurons, incluses dans la glume; grain étroitement enveloppé par la glumelle.....

4.

(1) a Locuste d'avoine cultivée ; $b$ locuste d'avoine élancée ; $c$ locuste d'avoine précoce. 
DEUXIÈyE PARTIE. - ANALYSES ET DESCRIPTIONS. 161

Paléole externe triaristée dont une $\{$ arête dorsale tordue. . . . . . . Avorne élancée.e.e.

Paléole externe bifide ou bidentée. 5 .

Panicule unilatérale contractée et Avone onirntale. très-fournie. ............ (c).

Plantes ne réunissant pas ces caractères. . . . . . . . . . . (c)

Paléole externe bidentée; locuste atteignant environ 12 millimètres de longueur; panicule presque unilatérale. ................ AvoIne courte (d).

Paléole externe bifide; locustes ayant plus de 12 millimètres de lon- Avorne cultrvée gueur; panicule régulière...... (b).

6.

Paléole externe biaristée au som\{met et munie d'une arête dorsale. . . Avorne hérissée(f).

Paléole externe bifide et munie (d'une arête dorsale. . . . . . . Avoine folde (g).

f Paillettes à 5-11 nervures; ovaire glabre................... Avorne ténue (h). Paillettes à 1-3 nervures. . . . . 9.

Rameaux de la panicule portant 1-3 locustes; ovaire poilu. ....... Rameaux de la panicule portant $5-8$ (locustes ou plus; oraire glabre. . . . 10. 11 ,

Scobine chargée de poils courts; locustes à $4-8$ fleurons. .......... Avorne des prés (i).

Scobine chargée de poils qui égalent presque la moitié de la longueur du Avorne pubescente fleuron. ............. (j).

Arête épidorsale; feuilles planes, Avoine jaunatre assez longues. ....................

Arête hypodorsale; feuilles trèsétroites. . . . . . . . . .

(k).

12.

Panicule spiciforme, oblongue. . Avorne PRÉCOCE(I). Panicule à rameaux allongés, étalés. Avorse CarvopHul.ÉE $(m)$. 
$\S$ I. Locustes pendantes; paillettes à 5̆-11 nervures; ovaire plus ou moins poilu.

A. - Paléole externe glabre ou nunie de QuelQUES POILS ÉPARS; SCOBINE GLABRE OU PRESQUE gLAbre, oU COUverte a LA BASE DE POILS COURTS.

a) Avoine nue. (Avena nuda L.) Chaume de 9 à 17 décimètres, droit, fort; feuilles glabres, striées; panicule régulière; locustes à 2-8 fleurons, plus longues que la glume; palćoles membraneuses, glabres, se séparant spontanément du grain à la maturité; fleuron supérieur toujours mutique; grain pubescent au sommet, glabre dans le reste de son étendue. Annuelle; fleurit en juillet. On cullive deux variétés d'avoine nue, savoir :

La petite avoine nue, dont la presque totalité des grains'se dépouillent de la balle et dont le grain est petit, roux, et les paléoles jaunâtres;

La grosse avoine nue, dont un grand nombre de grains ne se dépouillent pas de la balle; panicule large, étalée; paille forte; paléole grise noirâtre; grain blane bien nourri, deux fois aussi fort que le précédent.

b) Avoine cultivée. (Avena sativa L.) Chaume de neuf à dix-septdécimètres, droit, ferme, glabre; feuilles larges, planes, un peu rudes au toucher; panicule régulière étalée; rameaux semi-verticillés, hispides, les uns divisés, lesautres simples; locustes à deux ou trois fleurons fertiles, plus courtes que la glume; paillettes à onze nervures; fleuron supérieur neutre; paléole extérieure lancéolée, bifide ou bidentée au sommet, présentant à la base un petit 
faisceau de poils courts; arête longue, rousse à la base et tortillée, ou toutes les fleurs mutiques, à cause de la caducité de l'arête, provoquée par la culture; grain étroitement renfermé entre les paléoles. Annuelle; fleurit en juillet et aoùt.

Parmi les nombreuses variélés sur lesquelles nous avons acquis des données expérimentales, on peut signaler :

$1^{\circ}$ L'avoine blanche des Flandres. - Grain (1) très-allongé, assez maigre; balle d'un blanc jaunâtre; paille élerée.

2. L'avoine patate. - Grain blanc, court, pesant, à balle fine peu coriace; panicule grande, à locustes abondantes; pédoncules droits ou à peine un peu flexueux. 'Tardive.

$3^{\circ}$ L'avoine de Kamschatka ou de Sibérie. Grain blanc-jaunâtre, gros, pesant ; balle épaisse, subcoriace, dure; panicule très-grande à rameaux supérieurs et latéraux flexueux. Très-précoce.

Cette variété présente une sous-variété qui se rapproche beaucoup de la précédente; elle ne s'en distingue que par sa panicule moins forte et fournie, et tout aussi précoce qu'elle. Elle est connue depuis longtemps sous le nom d'avoine de Géorgie; toutes deux conservent longtemps leur grain.

$4^{\circ}$ L'avoine de Hopetown. - Grain blanc assez court, un peu renflé; balle épaisse subcoriace, dure; paille très-élevée; panicule longue; fournie. Très-tardive.

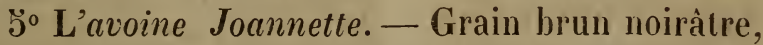
un peu allongé et renflé, bien nourri ; balle fine, subpapyracée. Elle s'égrène assez promptement.

(1) Le grain d'avoine, quoique enveloppé de la glume ou bal'e, est désigné dans le commerce et dans les descriptions agricoles sous le nom de grain. 
$6^{\circ}$ L'avoine de Brie. - Grain brun-noirâtre, assez court, renflé. Cette variété a beaucoup d'analogie avec la précédente et s'en distingue assez difficilement. M. Vilmorin lui assigne comme caractère distinctif subsidiaire qu'une partie des grains ne se séparent point au battage et restent attachés deux ensemble par leur base.

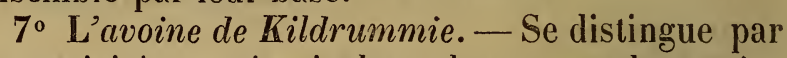
sa rusticité et réussit dans les terres les moins fertiles; mais son rapport est loin de pouvoir être comparé à celui des espèces précédentes.

$8^{\circ}$ L'avoine de Frise. - Se rapproche beaucoup de celle des Flandres; mais elle ne vient bien que dans les terres sablonneuses fraiches.

c) Avoine orientale. (Avena orientalis Schreb.) Chaumes de neuf à dix-huit décimètres, glabres, lisses, gros; feuilles larges, glabres, striées; panicule unilatérale, à rameaux les uns simples, les autres divisés ; locustes plus courtes que la glume, à deux fleurons fertiles, souvent accompagnés d'une troisième fleur non développée; un des fleurons fertiles mutique; arête presque droite, peu genouillée; paillettes marquées de neuf à onze nervures; paléole externe bidentée ou bifide, non biaristée.

On en cultive deux variétés, la blanche et la noire. La noire est plus productive et de meilleure qualité dans les bons terrains que la blanche, qui réussit même assez bien dans les plus médiocres. Annuelle; fleurit en juillet.

d) Avoine courte. (Avena brevis Roth.) Chaume de neuf à dix-huit décimètres, droit; feuilles planes, glabres, plus ou moins glauques, ou couvertes d'une espèce de poussière grisâtre; panicule égale ou presque unilatérale, étalée; locustes à deux fleu- 
rons fertiles aristés; paléole externe bidentée au sommet et plus ou moins hérissée, poilue supérieurement; paillettes de sept à neuf nervures; arêtes scabres; grain très-obtus au sommet. Annuelle; fleurit en juillet.

e) Avoine élancée. (Avena strigosa Schreb.) Chaume de sept à quinze décimètres, droit, arrondi, strié, glabre; feuilles planes, rudes; panicule unilatérale ou presque unilatérale, droite, contractée ou étalée, légèrement penchée au sommet, assez garnie; locustes à deux ou trois fleurons, aussi longues que la glume; paillettes lancéolées, aiguës, l'intérieure à sept ou neuf nervures; paléoles glabriuscules, lancéolées, atténuées au sommet, l'extérieure bifide à deux arêtes subterminales, droites, ayant environ le tiers de la longueur de la paléole et portant une arête dorsale brun-noirâtre peu tordue dans son milieu, deux fois aussi longue que la glume.

On en cultive deux variétés, l'une à balle jaunâtre et l'autre à balle brun-noirâtre ; elle est glabre ou hérissée supérieurement. Annuelle ; fleuit en juillet.

B. - palÉole externe COUverte dans sa moitié iNFÉRIEURE DE LONGS POILS; SCOBINE pOILUE.

f) Avoine hérissée. (Avena hirsuta Nob.) Chaume de huit à quinze décimètres, glabre; feuilles striées, larges, ciliées; panicule égale ou presque égale; locustes à deux ou trois fleurons, tous aristés; paléole extérieure couverte de poils longs, blanc-roussâtre, et hiaristée au sommet. Annuelle, fleurit de juillet à septembre. 
Parmi les avoines cultivées, notamment dans les provinces de Liége, de Hainaut, et de Luxembourg.

g) Avoine folle. (Avena fatua L.) Chaume de 9 à 15 décimètres, dressé, glabre; feuilles planes, striées, larges; panicale étalée, régulière ou presque unilatérale; rameaux semiverticillés, souvent flexueux, hispides; locustes de deux à quinze fleurons, plus courtes que la glume; paléole externe bidentée au sommet, munie d'une arête dorsale longue, tordue, genouillée dans son milieu et garnie dans sa moitié inférieure, au moins, de poils longs, abondạnts, roides, blanchâtres dans leur jeunesse, roussâtres à la maturité.

On a obtenu en Ecosse une variété d'avoine folle qui se distingue par ses paléoles munies de quelques rares poils roussâtres.

Nous considérons l'avoine stérile (avena sterilis L. ) comme une variété de l'avoine folle. Annuelle; fleurit en juin et juillet.

$\S$ II. Locustes non pendantes; paillettes de cinq à onze nervures; ovaire glabre ou à peine poilu au sommet.

h) Avoine ténue (Avena tenuis Moench.) Chaumes rameux à la base, roides, souvent genouillés vers le bas, un peu rudes, surtout supérieurement; feuilles étroites, linéaires ; ligule oblonguelancéolée, souvent laciniée; panicule régulière à rameaux semiverticillés, simples ou divisés; 10 custes de trois à cinq fleurons dépassant la glume; fleuron inférieur muni d'une arête terminale; les autres biaristées au sommet et pourvues d'une arête dorsale fortement genouillée vers le niveau de la terminaison des arêtes terminales; scobine poilue aux noeuds. Annuelle; fleurit en juillet. 
DELXIÈME PIRTIE. - ANALYSES ET DESCRIPTIONS. 167

$\S$ III. Locustes non pendantes; paillettes pourvues de une à trois nervures.

G Rameaux de la panicule portant une à trois locustes; ovaire poilu au sommet.

i) Avoine des prés. (Avena pratensis L.) Chaume de quatre à sept décimètres, droil, roide, glabre, garni à la base de faisceaux de feuilles droiles, rudes en dessus, glabres, subpliées ou roulées en long; les caulinaires à limbes courts, ordinairement planes et à gaînes très-allongées; panicule resserrée en épi, droite; rameaux inférieurs verticillés, les uns très-courts portant une locuste, les autres plus longs en portant deux ou trois, ou locustes solitaires ou géminées, portées sur des rameaux plus ou moins longs ou subsessiles ; locustes de quatre à neuf fleurons; arête presque tétragone, droite inférieurement, ensuite tortillée, souvent divariquée, presque constamment panachée de noir et de blanchâtre.

On distingue les variétés suivantes :

A voine des prés faux brome. Rameaux de la panicule verticillés, les uns très-courts ne portant qu'une locuste, les plus longs en portant deux ou trois; locustes de quatre à six fleurons; paléole externe munie à la base d'un petit renflement poilu.

Avoine des prés vraie. Locustes solitaires ou géminées à sept ou huit fleurons; paléoles glabres ou presque glabres à la base. Vivace; fleurit en juin et en juillet.

Coteaux arides et bois montueux.

j) Avoine pubescente. (Avena pubescens L.) Chaumes de cuatre à huit décimètres, assez grêles; feuilles courtes, planes; ligule tronquée ou aiguë, non ciliée; panicule plus ou moins étalée, régulière, à rameaux disposés par 5-ơ dans le bas, 
géminés ou solitaires dans le haut; locustes à deux ou trois fleurons aristés; scobine desfleurons supérieurs chargée de poils qui égalent presque la moitié de la longueur des paléoles; arête longue, fléchie et contournée vers son milieu, d'une couleur plus ou moins violette à la base.

Il y a une variétéà feuilles et gaînes pubescentes et une autre à feuilles et gaînes glabres. Vivace, fleurit en mai et en juin.

Dans les prés et les pâturages secs et sablonneux. $b$ Rameaux de la panicule portant de cinq à huit locustes ou plus;
ovàire glabre.

k) Avoine jaunatre. (Avena flavescens L.) Chaume de cinq à douze décimètres, assez grêles, droits, garnis de feuilles planes, larges, glabres ou plus ou moins pubescentes; gânes inférieures velues; ligule très-courte, tronquée et presque toujours ciliée ou denticulée, lacérée; panicule étalée, multiflore; locustes jaune-verdâtres, de deux à cinq fleurons aristés et luisants; scobine un peu velue; paillettes inégales, comprimées, rudes vers le sommet; paléole externe bifide à divisions aiguës, à peine velue, à cinq nervures, pourvue d'une arête naissant au-dessus de la partie moyenne de la paléole; arête courbée, pliée ou genouillée après la floraison. Vivace; fleurit en juin et en juillet.

Dans les prés.

m) Avoine précoce. (Avena procox P. D. B.) Chaumes de deux à quatre décimètres, disposés en touffes menues, vertes, lisses, un peu couchés, garnis dans le bas de quelques feuilles courtes et sétacées; gaines anguleuses; liguleallongée; locustes dressées; panicule spiciforme, oblongue, compacte, n'atteignant presque jamais au delà de deux centi- 
DEUXIĖuE PARTIE. - ANALYSES ET DESCRIPTIONS. 169

mètres de longueur; locustes biflores d'un vert blanchàtre; paillettes à une nervure; paléole externe munie d'une arète un peu genouillée, insérée au-dessous du milieu de la longueur de la paléole. Annuelle; fleurit de mars à juin.

Croît fréquemment dans les lieux sablonneux et stériles.

n) Avoine caryophillée. (Avena caryophillea. Wigg.) Plante renant en petites touffes d'une couleur glauque; chaumes de un à trois décimètres, très-fins et droits; feuilles courtes et sétacées; ligule très-allongée, bifide, à divisions trèsaiguës; panicule presque toujours trichotome; locustes biflores, rassemblées au sommet des ramifications; les pédoncules souvent plus courts que les locustes qui contiennent deux fleurons, à paléoles externes bifides, aiguës, à une nervure glabre ou presque glabre.

Il y a une variété à fleuron supérieur stipité et une autre à fleuron supérieur sessile ou subsessile. Annuelle; fleurit en juin et juillet.

Lieux secs, élevés el sablonneux.

$$
\text { GENRE 29. - CANCHE. (AIRA L.) }
$$

Locustes comprimées latéralement, à 2 fleurons accompagnés parfois d'une troisième fleur rudimentaire; paillettes carénées, mutiques, presque égales; paléole externe munie de trois à cinq dents irrégulières au sommet, et présentant vers le bas une arête géniculée ou droite, plus ou moins tordue inférieurement; paléole intérieure bicarénée; paléolules 2; ovaire glabre; styles courts, naissant au-dessous du sommet; stigmates 2 ; grain oblong, comprimé non sillonné; panicule rameuse. 

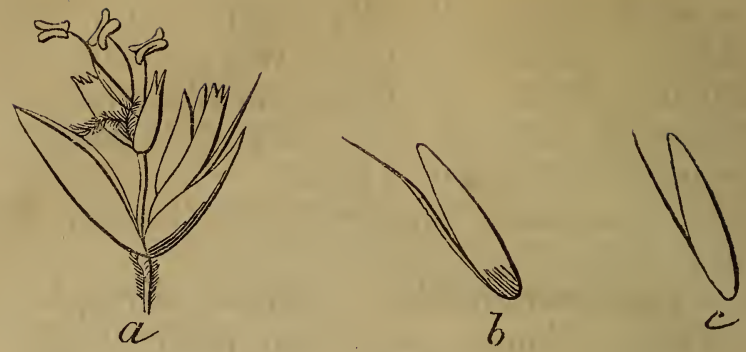

Fig. 55 (1).

ANALYSE DES ESPÈCES.

(Arête droite, ne dépassant pas ou dépassant à peine la paléole; feuilles Canche cespiteuse. 1 Arête dépassant la glume, genouillée; feuilles filiformes. 2. (Ligule très-longue, acuminée. . Canche Discolore(b). 2 Ligule tronquée ou obtuse, non acu-Canche flexueuse. minée........... (c).

a) Canche cespiteuse. (Aira cospitosa L.) Plante formant ordinairement des toufies de gazon serrées et arrondies; chaumes de sept à treize décimètres, peu feuillés, droits, durs, striés, lisses; feuilles planes, striées et rudes en dessus et sur les bords, lisses en dessous; ligule allongée, fendue; panicule étalée à rameaux rudes; locustes à deux ou trois fleurons ordinairement luisants; paléole extérieure dentée au sommet, à cinq nervures; arête presque basilaire, à peine tordue à la base et droite ou presque droite, ne dépassant pas la glume.

Cette plante présente trois variétés principales : la première, qui constitue une espèce pour quelques

(1 a Locuste de canche cespiteuse; $b$ paléole de la canche flexueuse ; c paléole de la canche cespiteuse, vue de profil. 
auteurs, que l'on désigne sous le nom de canche à petite fleur, se distingue par seslocustes dont le fleuron supérieur ne dépasse pas la glume et par sa paléole externe qui ne présente qu'une seule nervure, tandis qu'il en existe deux ou trois plus ou moins distinctes dans la canche cespileuse. La seconde, qui est connue par quelques agronomes sousle nom de canche naine (aira minor) a un chaume faible et des feuilles enroulées. La troisième, enfin, sous le nom de grande canche (aira major), présente des feuilles planes, une panicule variée de vert et de violet ou de jaunàtre, et des locustes assez grandes, dont le fleuron supérieur, ainsi que l'arête, dépasse un peu la glume. Vivace; fleurit en juin et en juillet.

Endroits un peu humides aux bords des fossés, bois ombragés.

b) Canche discolore. (Aira discolor L.) Chaumes droits de quatre à neuf décimètres, glabres, grêles, presque nus ; feuilles la plupart radicales, minces, filiformes, glabres, fort longues; ligules très-longues, acuminées; panicule plus ou moins étalée ; rameaux filiformes plus ou moins flexueux; locustes souvent rougeàtres; arète coudée dépassant la glume; scobine qui supporte le fleuron supérieur, de moitié environ plus courte que lui. Vivace; fleurit d'août à septembre.

Dans les bois-taillis, les marais tourbeux, les eaux tourbeuses.

c) Canche flexueuse. (Aira flexuosa L.) Chaumes de quatre à huit décimètres, droits, s'élevant d'un gazon serré; feuilles très-minces et capillaires; les ligules des feuilles caulinaires tronquées ou trèsobtuses, presque toujours bifides ou multifides, à divisions très-obtuses ou tronquées; panicule peu fournie, subtrichotome, étalée, ou plus ou moins 
resserrée, à rameaux capillaires, un peu rudes; paillettes luisantes, souvent panachées de blanc argenté et de violet; paléole externe à cinq nervures, garnie à sa base d'un faisceau de poils courts et d'une arète assez longue dépassant la glume, coudée dans son milieu; scobine supportant le fleuron supérieur au moins quatre fois plus courte que lui, ou fleuron subsessile.

On connaît plusieurs variétés locales qui sont dues à la position, à la situation, au sol et au climat dans lesquels elles se présentent. Il en est une à locustes blanchâtres; les autres sont panachées de blanc et de violet, mais la panicule est tantòt très-large et étalée, et d'autres fois elle est plus ou moins contractée et à rameaux droits. Vivace; fleurit d'août à septembre.

Abonde dans les bois ombragés et sablonneux.

ve tribu. - Ampénathémacées.

Les arrhénathéracées présentent une panicule rameuse, étalée ou spiciforme, des locustes à deux ou trois fleurons dont un hermaphrodite et un ou deux mâles, ou bien des locustes contenant deux paléoles aristées; les paillettes sont carénées et les stigmates sortent vers la partie inférieure des glumelles, rarement au sommet du fleuron.

ANALYSE DES SOUS-TRIBUS.

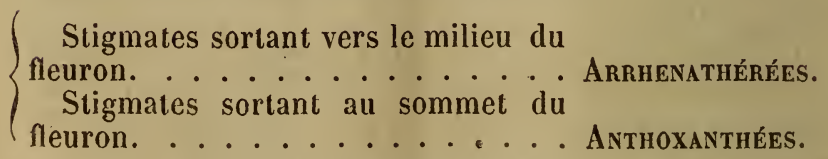

SUCCESSION.

10 Arrhénathérées.

2० Anthoxanthées. 
DEUXIẺME PARTIE. - ANALYSES ET DESCRIPTIONS. 173

I cr sous-tribu. - Arrhénatérées.

Stigmates sortant vers le milieu du fleuron.

genre 50. - ARrhénathère. (Arrhenatherum F. D. B. )

Locustes biflores présentant presque toujours un troisième fleuron rudimentaire réduit à un pédicelle grêle; fleuron supérieur hermaphrodite, fleuron inférieur mâle; paillettes convexes mutiques, l'intérieure égalant la longueur des paléoles.

Fleuron mâle; paléole extérieure convexe, portant une arête dorsale genouillée, tordue inférieurement.

Fleuron hermaphrodite : paléole extérieure mutique ou munie d'une arête courte presque terminale; paléolules 2, lancéolées, entières ou unidentées latéralement; ovaire turbiné, poilu au sommet; stigmates sessiles terminaux, plumeux, sortant vers la partie inférieure ou vers le milieu du fleuron; grain sillonné sur l'une de ses faces; panicule rameuse, étalée.

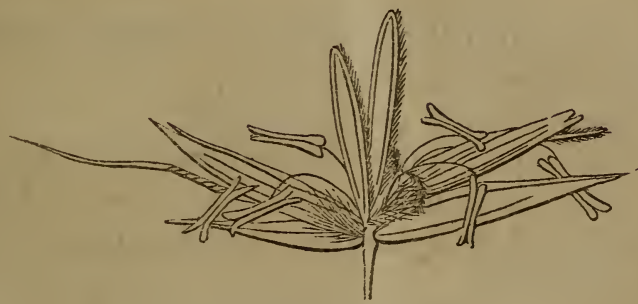

Fig. 34 (1).

(1) Locuste d'arrhénathère fausse avoine. 
Arrhénathère fausse avoine. (Arrhenatherum avenaceum P. D. B.) Souche cespiteuse ou un peu traçante émeltant beaucoup de chaumes de sept à quinze décimètres, droits ou un peu obliques supérieurement, glabres ou velus, surtout sur les nouds; feuilles assez longues, planes; panicule plus ou moins oblongue, un peu étroite et souvent penchée à la floraison; locustes à deux fleurons développés, l'un ou tous les deux aristés; le fleuron inférieur muni d'une longue arête coudée et tortillée dañs son milieu; glume et glumelle d'une couleur plus ou moins jaune violacée.

L'arrhénathère fausse avoine offre plusieurs variétés dont les caractères sont assez constants; ce sont :

$1^{\circ}$ L'arrhénathère bulbeuse, qui présente un rhizome composé d'une ou de plusieurs nodosités ou tubérosités placées les unes sur les autres, contiguës et disposées comme les grains d'un chapelet;

$2^{\circ} L^{\prime}$ 'arrhénathère rupestre, dont le rhizome est granulé, le chaume et les feuilles glauques et le fleuron supérieur très-relu ;

$3^{\circ}$ L'arrhénathère $\dot{a}$ une arête; rhizome noueux ou tubéreux ; fleuron hermaphrodite mutique;

$4^{\circ}$ L'arrhénathère élevée, à rhizome noueux et à deux fleurons aristés.

Vivace; fleurit de juin à août.

Dans les prés secs ct un peu humides; assez fréquent aux bords de l'eau, dans les lieux ombragés; les variétés 1 et 2 dans les endroits secs. 
DEUXIÉME PARTIE. - ANALYSES ET DESCRIPTIGNS. $17 ̈ 0 ̈$

GeNre 51. - HOUQUE. (HOLCUS L.)

Locustes polygames, biflores; scobine droite ; fleuron inférieur hermaphrodite; fleuron supérieur mâle; glume à paillettes comprimées, carénées, presque égales, à trois nervures, enveloppant la locuste.

Fleuron mâle; paléole extérieure carénée, portant une arête épidorsale plus ou moins tordueà la base; étamines 3 .

Fleuron hermaphrodite ; paléole extérieure mutique; paléoles 2, entières; étamines 3 ; ovaire glabre; stigmates terminaux, sessiles, plumeux, sortant vers la partie inférieure ou vers le milieu du fleuron; grain réniforme, arrondi ou oblong, non sillonné; panicule rameuse plus ou moins étalée.

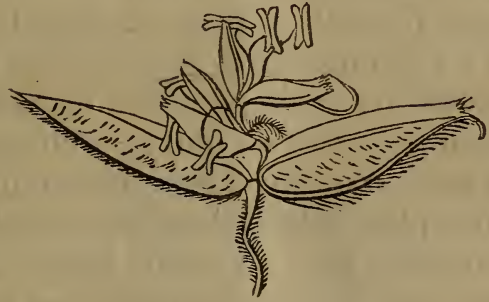

Fig. $5 \ddot{3}(\mathbf{I})$.

ANALYSE DES ESPÈCES.

Souche traçante; arête genonillée dépassant sensiblement la glume. Hovque nolle (a). Souche cespiteuse; arête recourbée en hameçon à la maturité, ne dépas- Houque caineuse sant pas la glume ......... (b).

(1) Locuste de houque laincuse, grossie. 
a) Houque molle. (Holcus mollis L.) Souche émettant des rhizomes longuement traçants ; chaume de cinq à douze décimètres, presque glabre, à nouds couverts de poils assez longs, blancs; feuilles planes, molles, un peu rudes sur les bords, avec quelques poils épars sur leurs gaînes; panicule étalée lors de la floraison, plus ou moins resserrée avant et après, d'une couleur d'abord verdâtre, puis roussâtre; paillettes très-aiguës, glabres ou velues, légèrement ciliées sur le dos; locustes plus courtes que la glume; fleuron supérieur muni à sa base de quelques poils blancs atteignant environ le tiers de sa longueur; arête d'abord droite, ensuite un peu coudée ou genouillée, dépassant sensiblement la glume, plus longue que la paléole qui la porte. Vivace; fleurit de juillet à septembre.

Dans les bois un peu humides et aux bords des champs, rarement dans les prés.

b) Houque laineuse. (Holcus lanatus L.) Souche cespiteuse; chaumes assez forts et plus ou moins droits et genouillés à la base, mollement pubescents; feuilles planes, douces au toucher, chargées, ainsi que leurs gaînes, d'un duvet cotonneux qui fait parajtre souvent la planteblanchâtre; panicule étalée pendant la floraison, plus ou moins colorée de violet, assez fournie; paillettes un peu arrondies au sommel, subaristées, velues et ciliées; locustes plus petites que la glume; paléole externe du fleuron supérieur glabre ou presque glabre, ou garni à la base de quelques poils extrêmement courts n'ayant jamais le tiers de sa longueur; arête en hameron à la maturité, ne dépassant pas ou dépassant à peine la glume et n'atteignant pas la longueur du fleuron. Vivace; fleuril de mai à novembre.

Prairies, bois. 
DEUXIÈME PARTIE. - ANALYSES ET DESCRIPTIONS. 177

Ir sous-tribu. - Anthoxanthées.

Stigmates sortant vers le sommet du fleuron.

GENRE 32. - HIEROGHLOË. ( IIEROChloË GMEL.)

Locustes triflores; scobine bipliée; fleurons latéraux mâles, fleuron intermédiaire hermaphrodite; glume à deux paillettes membraneuses enveloppant la locuste; fleuron màle; paléole extérieure entière ou fendue, portant une petite arête épidorsale; étamines 3 ; fleuron hermaphrodite; paléole extérieure mucronée; étamines 3 ; paléoles 2 , ordinairement entières; ovaire glabre; styles 2 , dressés; stigmates en goupillon, divergents, sortant au sommet des glumelles; grain oblong ou un peu réniforme, arrondi, non sillonné ou à peine canaliculé ; panicule rameuse.

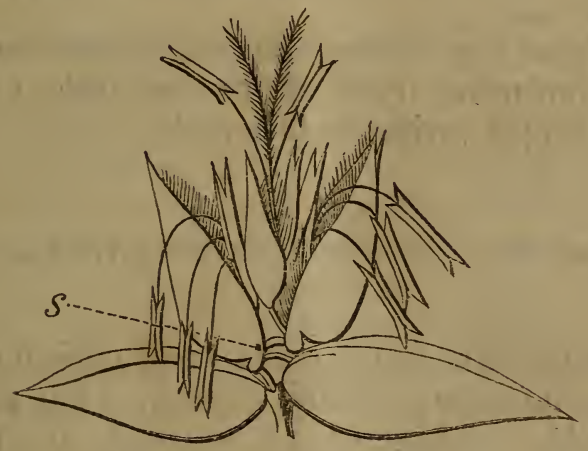

Fig. 56 (1).

ANALYSE DES ESPÉCES.

Rameaux de la panicule glabres. . H. BORÉALE. (a). Rameaux de la panicule velus. . II. austrack (b).

1) Locuste de thierochloë boreale, grossic. 
a) Hierochloé boréale. (Hierochloë borealis Gmel.) Souche traçante; chaumes grêles, garnis vers leur base de feuilles longues, étroites : souvent il n'existe sur le chaume qu'une longue - gaîne presque toujours ventrue, terminée par le rudiment d'une feuille avortée; paniculeà rameaux géminés, presque unilatérale, un peu lâche, peu garnie, de couleur le plus souvent jaunâtre, mêlée de brun ou de violet; rameaux glabres; paléole externe velue-ciliée, portant une arête courte subterminale. Vivace; fleurit de mai à juillet.

Prés humides du nord de la Belgique. (?)

b) Hierochloë australe. (Hierochloë australis Dmtr.) Celle-ci a beaucoup d'affinité avec l'espèce précédente ; elle ne s'en distingue que par ses rameaux qui sont velus et par la paléole externe des fleurons mâles fendue et portant une arête dorsale. (?)

Il se peut que ces deux espèces doivent leur origine à un même type et que l'australe ne soit qu'une simple variété de la boréale.

GeNRE 33. - FLoUVE. (ANThOXANThUM L.)

Locustes à une fleur fertile accompagnée de deux fleurons inférieurs réduits, chacun, à une paléole canaliculée, échancrée au sommet et munis d'arêtes, dont une au moins genouillée; paillettes carénées, l'extérieure de moilié plus courte que l'intérieure ; paléole externe du fleuron fertile beaucoup plus courte que la paléole des fleurons stériles, membraneuse, naviculaire et mutique; élamines 2 ; ovaire glabre; styles 2, longs, 
terminaux et soudés inférieurement; stigmates 2, allongés, plumeux, sortant au sommet du fleuron; grain oblong étroit, un peu comprimé, étroitement renfermé entre les paléoles; panicule spiciforme.

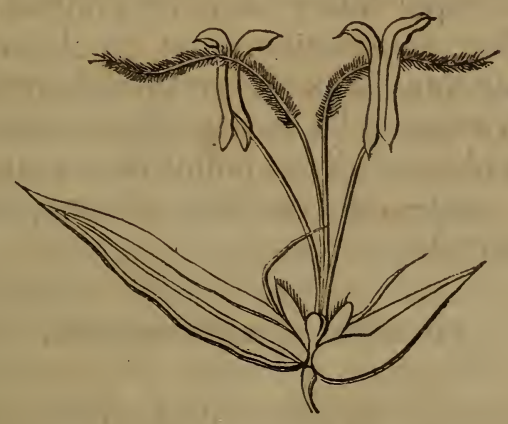

Fig. 57 (1).

Flouve odorante. (Anthoxanthum odoratum L.) Souche cespiteuse; chaumes de quatre à neuf décimètres, disposés en touffes simples, droits; feuilles planes, au nombre de deux à quatre sur le chaume, rudes sur les bords et un peu ciliées à la base, plus courtes que leurs gaines ; panicule spiciforme ovaleoblongue, compacte, rarement presque diffuse ; locustes légèrement pédonculées; paléoles des fleurons stériles, arrondies, obluses, velues, à poils appliqués, bruns, luisants, l'une portant une arête sétiformedroite, l'autre une arête tortillée à la base et genouillée, dépassant la glume.

Les variétés de la flouve sont peu nombreuses, nonobstant les stations diverses qu'on la voit occuper ; les caractères qu'elles revètent dépendent

(1) Locuste de flouve odorante, grossic. 
uniquement du sol qui leur imprime plus ou moins de vigueur et de développement. C'est ainsi que sur les hauteurs, la flouve prend des locustes velues et une panicule contractée; dans les sols fertiles de plaine, elle acquiert une panicule rameuse, lâche et presque étalée; dans les prairies irriguées ou humides, sa panicule, tout en devenant plus nourrie que dans la première variété, reste cependant contractée, et les locutes deviennent glabres. Quant au chaume, il est tantôt lisse et tantôt scabre dans toutes les variétés. Vivace; fleurit en avril, mai et juin.

\section{VI tribu. - Mgrostidicées.}

Les agrostidicées oflirent deslocustes à un fleuron fertile, parfois accompagné d'un rudiment, disposées en panicule étalée ou spiciforme, jamais digitée; paillettes carénées ou un peu arrondies et velues - ciliées, ou subplumeuses; stigmates 2 , sortant vers la partie inférieure ou moyenne du fleuron.

ANALYSE DES SOUS-TRIBUS.

$1\left\{\begin{array}{c}\text { Panicule rameuse plus ou moins } \\ \text { étáe. Aanicule spiciforme compacte. . A Amostidées. }\end{array}\right.$

SUCGESSION.

10 Agrostidées.

$2^{\circ}$ A Ammophilées. 
DEUXIĖyE P.ARTIE. - ANALYSES ET DESCRIPTIOXS. 181 Ire sous-tribu. - Agrostidées.

Panicule rameuse plus ou moins étalée.

GeNRE 34. - Calanagrostide. (CAlaMigrostis Adans.)

Locustes à un fleuron fertile accompagné parfois d'un rudiment pénicilliforme ; fleuron entouré à la base d'un involucelle de poils plus ou moins longs; paillettes plus longues que les paléoles qui sont inégales, l'extérieure plus grande, bifide, quadrifide ou à quatre dents obscures, munie d'une arête qui prend naissance au dos, au sommet ou dans l'échancrure de la paléole; paléolules 2, entières; ovaire glabre; stigmates terminaux, subsessiles, plumeux; panicule rameuse étalée ou lobée.
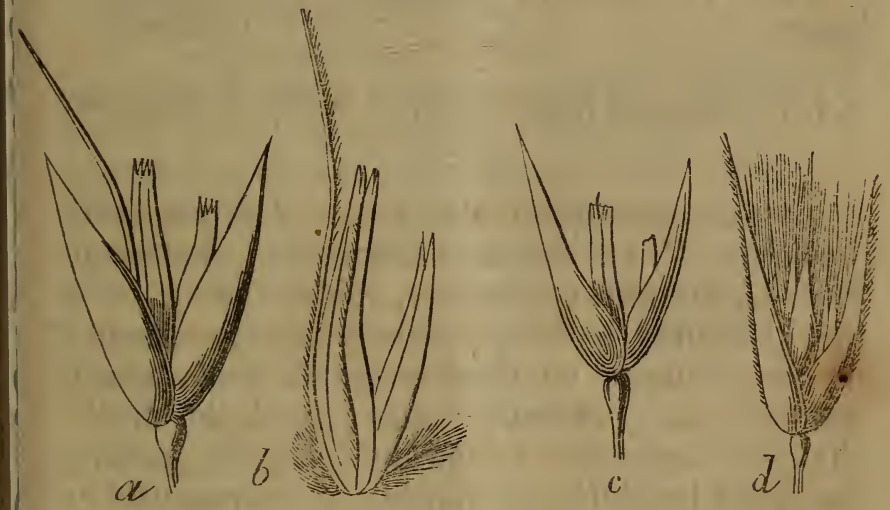

Fig. 38 (1).

(1) $a$ Locuste de la calamagrostide des bois ; $b$ fleuron de la mème ; $c$ locuste de la calamagrostide lancéolée; $d$ id. de la calamagrostide terrestre; toutes grossies. 


\section{ANALYSE DES ESPÈCES.}

$\int$ Un rudiment pénicilliforme à la Paléole interne dépourvue de rudiment pénicilliforme. ....... Arête dépassant longuement la glume; rudiment atteignant environ la moitié de la longueur de la paléole interne; Calamagrostide 2 poils environ quatre fois plus courts. DES BoIs (a). Arête dépassant à peine la glume; rudiment presque aussi long que la paléole interne...........

(Feuilles inférieures à gaînes poilues, notamment vers la partie supérieure; Calamagrostride Lligule oblongue-obtuse ....... des montagnes (b).

5 Feuilles inférieures à gaînes glabres ou presque glabres; ligule oblongue- Calamagrostide lancéolée ............. subulée (c). ¡ Arête dorsale. . . . . Calamagrostide terrestre (d).

4 Arête terminale ou émarginale, Calamagrostide courte. . . . . . . . . . . LancÉolée (e).

$\S$ I. Un rudiment pénicilliforme à la base de la paléole interne.

a) Calamagrostide des bois. (Calamagrostis sylvatica D. C.) Souche subtracante; chaume de douze à dix-huit décimètres, roide ; feuilles étroites, pointues; gaîne présentant supérieurement à la face externe un demi-cercle de poils courts disposés sur plusieurs rangs; panicule droite, étalée ou contractée, à rameaux fasciculés, scabres; paillettes lancéolées-acuminées, membraneuses en leurs bords; paléole extérieure à quatre dents au sommel, portant au dos une arêle genouillée dépassant longuement la glume; rudiment pénicilliforme atteignant environ la moitié de la longueur 
de la paléole; poils de l'involucelle peu abondants, quatre fois environ plus courts que le fleuron. Celte espèce a tantôt une panicule éialée ou fasciculée; d'autres fois la panicule est contractée. Vivace; fleurit en juillet-août.

Bois montagneux de la province de Liége et de Luxembourg.

b) Calamagrostide des montagnes. (Calamagrostis montana Host.) Souche traçante; chaume de douze à dix-huit décimètres, roide; feuilles étroites, pointues; gaines inférieures poilues, munies supérieurement à la face externe d'un demicercle de poils courts disposés sur plusieurs rangs ; ligule oblongue-obtuse; panicule étalée; paillettes acuminées; paléole externe portant une arête plus ou moins genouillée, dépassant à peine lá glume; poils de l'involucelle presque aussi longs que le fleuron; rudiment pénicilliforme presque aussi long que la paléole interne. Vivace; fleurit en mai.

Bois qui avoisinent l'Escaut.

c) Calamagrostide subulée. (Calamagrostis subulata Dmtr.) Souche traçante; chaumes de neuf à quinze décimètres, élevés, fort scabres ; feuilles et gainnes presque glabres; ligule oblonguelancéolée; panicule droite, étalée, roide; paléole externe munie d'une arête plus longue que le fleuron et les poils qui sont plus courts ou d'égale longueur que le fleuron; rudiment pénicilliforme. Vivace; fleurit de juin à août.

Sur les coteaux arides et boisés. 
§ II. Paléole interne dépourvue de rudiment pénicilliforme.

\section{a) ARÊTE DORSAI:E.}

d) Calamagrostide terrestre. (Calamagrostis epigeios Roth.) Souche tracante; chaumes de douze à dix-huit décimètres, droits, striés, plus ou moins rudes en-dessous de la panicule, un peu glauques, ainsi que les feuilles qui sont assez longues, glabres ou légèrement pubescentes; ligule scarieuse, fendue; panicule plus ou moins étalée ou lobée, souvent panachée de vert et de violet noirâtre; paillettes aiguës, lancéolées, membraneuses, un peu ciliées et rudes sur le dos, surtout vers le haut: l'extérieure à une et l'intérieure à trois nervures; paléoles membraneuses, transparentes; poils de l'involucelle plus longs que le fleuron; arête plus courte que les poils ou de la même longueur environ qu'eux, naissant vers le tiers inférieurde la paléole. Vivace; fleurit de juillet à août.

Sur les coteaux secs, sablonneux, arides, et dans les bois montagneux.

b) ARÈte terminale ou ÉMARgiNale courte.

e) Calamagrostide lancéolée. (Calamagrostis lanceolata Roth.) Souche traçante; chaumes rameux, assez faibles, rudes, très-garnis de feuilles à la base ; feuilles très-étroites, roulées et finissant en pointe au sommet; panicule étalée; paillettes longues, inégales, hispides sur le dos; paléole externe portant une arête très-courte qui prend naissance dans l'échancrure de la paléole; poils de l'involucelle plus longs que la paléole extérieure. Vivace; fleurit en juillet et en août.

Dans les prairies humides, paludeuses, aux bords des fossés et des rivières. 
vers sa base ; paillettes carénées, enveloppant complétement le fleuron; paléoles glabres ou munies de quelques faisceaux de poils extrêmement courts; paléole externe mutique ou portant une arête dorsale ou presque terminale; l'interne bicarénée, quelquefois très-petite ou nulle; paléolules 2 , entières ou presque entières; ovaire glabre; styles très-courts, terminaux; stigmates 2 , plumeux ; panicule rameuse à rameaux inférieurs verticillés.

\section{ANALYSE DES ESPÈCES.} ${ }_{1}\left\{\begin{array}{c}\text { Un rudiment pédicelliforme à la } \\ \text { base de la paléole interne. }\end{array}\right.$

Pas de rudiment pédicelliforme à la base de la paléole interne . . . . .

Paléole externe très-aiguë, mutique. Agrostidedu Miexi2 Plante vivace. . . . . . . .

Paléole externe munie d'une arête (naissant au-dessous du sommet. . . .

Panicule ample, étalée; anthères li- Agrostide Jovet

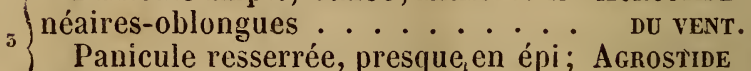
Panicule resserrée, presque en épi; Agrostrde inter-
anthères ovoïdes........... rompue.

(Feuilles radicales étroites, enroulées; paléole externe nulle ou très-

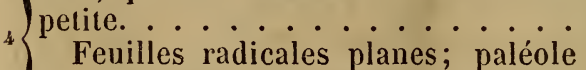
interne un peu plus petite que l'ex-

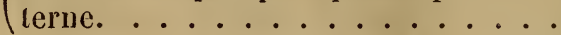

Agrostide des CHÊNES.

5.
Ligule des feuilles inféricures très- courte, tronquée; panicule plus ou
s moins étalée, même après la floraison. Agrostide vul- Ligule oblongue; panicule étroite, GaIRE. i contractée après la floraison. . . . . . Agrostide blanche 
§I. Une arête naissant un peu au-dessous du sommet, trois à six fois plus longue que la locuste, et un rudiment pédicelliforme à la base de la paléole interne. Plante anuuclle. (Voir fig. 39, lettres $a$ et $b$.)

a) Agrostide épi du vent. (Agrostis spica venti L. ) Chaumes de huit à quinze décimètres, droits, glabrès, striés, présentant de trois à six nœuds; feuilles rudes en leurs bords, toutes linéaires, planes; gaînes striées longitudinatement ; ligule oblongue; panicule ample; rameaux très-divisés et capillaires, étalés pendant la floraison et se redressant ensuite; verticilles inféricurs de la panicule recouvrant en partie le verticille qui est audessus; paillettes lancéolées, aiguës, hispides; paléole externe munie d'une arête un peu au-dessous du sommet, dépassant au moins la glume de trois à quatre fois la longueur de celle-ci; paléole interne présentant un rudiment pédicelliforme; anthères linéaires-oblongues; grain blanc et terne. Une variété présente une panicule plus ou moins contractée. Annuelle; fleurit en juin et juillet.

Dans les moissons $\mathrm{et}$ aux bords des champs.

b) Agrostide interrompue. (Agrostis interrupta L.) Chaumes de cing à neuf décimètres, droits au sommet, présentant un ou deux nœuds, rarement trois ; feuilles étroites; gaines longues; ligule oblongue; panicule longue, atténuée, filiforme, composée de verticilles dont les rayons sont très-inégaux; le verticille inférieur ne recouvrant que peu le verticille qui est au-dessus; verticilles très-rapprochés dans le haut, simulant un épi; paléole externe munie d'une arête trois à cinq fois plus longue que la locuste; paléole interne munie à sa base d'un rudiment pédicelliforme, tronqué; 
anthères ovoïdes. Annuelle; fleurit en juin et juillet.

Dans les moissons, les terres sablonneuses et sur les vieux murs. (?)

§ II. Paléole externe mutique très-aiguë ; paléole interne présentant à sa base un rudiment pédicelliforme. Plantes vivaces. (Voir fig. 39, lettres $\dot{c}$ et $d$.)

c) Agrostide du Mexique. (Agrostis mexicana L.) Chaumes de sept à quinze décimètres, droits; feuilles radicales et caulinaires planes; ligule courte, souvent subpectinée ou frangée; locustes disposées en glomerules allongés, plus ou moins compactes, qui donnent à la panicule une apparence quasi-lobée, resserrée avant et après la floraison; paléole externe mutique, couverte à la base de quelques poils qui ne dépassent presque jamais le tiers de la longueur du fleuron; paléole interne présentant à la base un rudiment pédicelliforme quelquefois tortueux, atteignant souvent la longueur de la paléole.

Cette plante, originaire des prés montueux du Mexique, est propagée çà et là dans les prairies de la Belgique. Vivace; elle fleurit de mai à août.

§ III. Paléole externe mutiquc ou aristée, l'interne dépourvue de tout rudiment pédicelliforme. Plantes vivaces.

d) Agrostide vulgaire. (Agrostis vulgaris Withering. ) Souche cespiteuse ou traçante;chaumes de quatre à treize décimètres, dressés ou obliques-ascendants, souvent radicants inférieurement; feuilles radicales et caulinaires presque toujours planes ; ligule des feuilles inférieures très-courte, tronquée; panicule à rameaux plus ou moins diffus, 
divariqués ou étalés même après la floraison; locustes souvent rougeâtres ou violacées; paillettes presque égales, aiguës, souvent un peu hispides sur la carène; paléoles 2 , l'intérieure un peu plus grande, presque toujours mutique.

Cette espèce présente un grand nombre de variétés dont les caractères distinctifs sont des plus obscurs; nous nous bornerons à signaler les plus importantes, qui sont :

L'A grostide vulgaire. Panicule d'un vert violacé, étalée.

L'Agrostide jaunâtre. Panicule très-lâche ; locustes d'un jaune verdâtre.

L'A grostide des bois. Paillettes et paléoles trèsallongées et locustes vivipares. Vivace; fleurit en juillet-aoùt.

Dans les prés, aux bords des fossés et dans les bois de toute la Belgique.

e) Agrostide blanche. (Agrostis alba With.) Souche cespiteuse ou traçante; chaumes de quatre à treize décimètres, dressés ou obliques-ascendants, souvent radicants ou stolonifères; feuilles radicales et caulinaires à ligule oblongue; panicule plus ou moins étalée, à rameaux dressés après la floraison ; locustes verdâtres ou violacées; paillettes presque égales, aiguës, souvent un peu hispides sur la carène; paléoles 2, l'extérieure un peu plus grande, presque toujours mutique; locustes rarement vivipares. Parmi les variétés que présente cette espèce, nous croyons devoir signaler :

L'Agrostide tracante. Souche émeltant III grand nombre de rhizomes longuement traçants; chaume ferme, un peu coudé aux nouds;

L'Agrostide stolonifère. Souche cespileuse ; chaumes couchés, faibles; 
L'Agrostide maritime. Chaume de quatre à huit décimètres, droit; panicule contractée ;

L'A grostide géant, qui se distingue par l'ampleur de toules ses partieset par sa panicule très-fournie d'un vert pàle. Vivace; fleurit de juin à septembre.

Dans les prés, les endroitsinondéspendantl'hiver, aux bords des chemins et dans les sables maritimes.

Les deux espèces qui précèdent offrent une variété dont la paléole est aristée au dos.

f) Agrostide des chiens. (Agrostis canina L.) Souche cespiteuse ou traçante, émettant fréquemment des rejets stériles non radicants; chaumes dressés ou ascendants, ou couchés à la base; feuilles radicales étroites, roulées, sétacées, les caulinaires petites, assez étroites, le plus souvent planes, parfois roulées; ligule oblongue, souvent déchirée; panicule rameuse très-variable, ordinairement resserrée avant et après la floraison, à rameaux rudes, à ramifications souvent très-fines et tortueuses; locustes violacées, rarement verdâtres; paillette externe plus grande et plus forte que lïnterne; paléole extérieure portant une arête hypodorsale un peu coudée, une ou deux fois plus longue que la locuste, rarement mutique; paléole intérieure très-pelite et souvent nulle. Vivace; fleurit de juillet à septembre.

Endroits humides, tourbeux et marécageux.

Ir sous-tribu. - Ammophilées.

Panicule spiciforme compacte.

genre 36. - lagurier. (lagurus L.)

Locuste à un fleuron fertile accompagné d'un rudimentétroit, a!longé, plus ou moins cilié; pail- 
lettes presque égales, très-longues, étroites, veluesciliées, comme plumeuses; paléole extérieure subcarénée, terminée par deux arêtes, et portant un peu au-dessous du milieu de sa longueur, une arête plus ou moins tordue à sa base; l'intérieure entière et mutique ou un peu acuminée ; étamines 5 ; paléolules 2, entières, ou un peu dentées, ou dechiquetées en leurs bords, glabres, un peu renflées à leur base; ovaire turbiné, styles 2 , courts ; stigmates 2 ; grain allongé, non sillonné ou à peine canaliculé; panicule spiciforme compacte, molle.

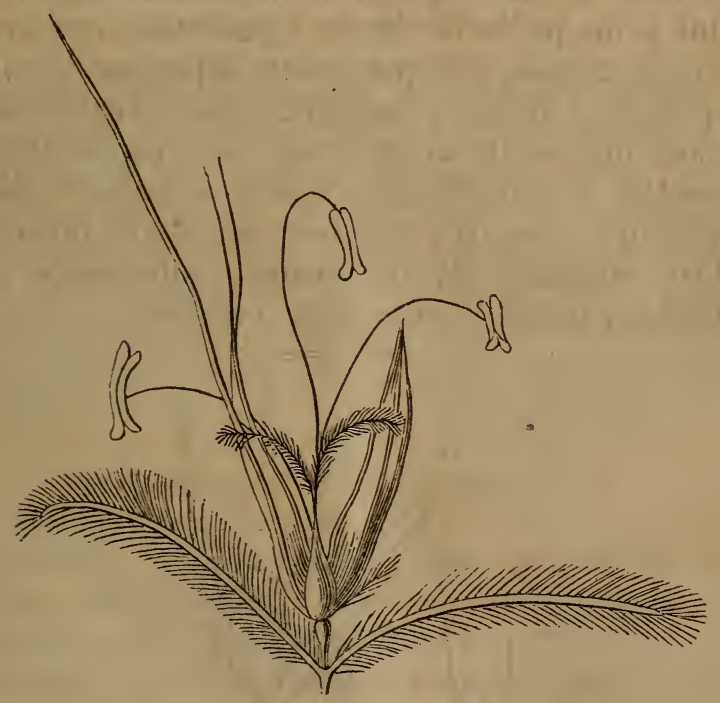

lig. 40 (1).

Lagurier ovale. (Lagurus ovalus L.) Chaume de 5 a 7 décimètres, rameux, glabre ou glabrius-

(1) Loctisle de lagurier orale, grossie. 
cule; feuilles molles, pubescentes; gaines blanchâtres, la supérieure renflée ; panicule contractéespiciforme, ovale-oblongue, rarement un peu sphéroïdale, très-velue, blanchâtre ou roussâtre. Cette espèce présente une variété presque entièrement glabre. Annuel; fleurit en juin et juillet.

Champs secs et sablonneux du Limbourg.

GENRE 37. - AMMOPHILE. (AMMOPHILA HOST.)

Locustes à un fleuron fertile entouré de poils à sa base et accompagné d'un fleuron supérieur' réduit à un pédicelle barbu; paillettes entières, carénées, aiguës, presque égales, dépassant à peine les paléoles; paléoles presque égales, l'extérieure portant une petite arête au-dessous du sommet; paléolules 2, entières, lancéolées-acuminées, plus longues que l'ovaire; étamines 3 ; ovaire turbiné, glabre; stigmates 2, terminaux, subsessiles, en goupillon; panicule spiciforme compacte.

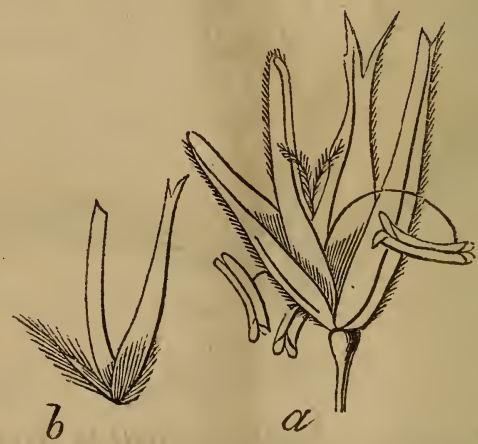

Fig. 41 (1).

(1) a Locuste d'ammophile des sables; $b$ flcuron du mème, grosisis. 
Ammophile des sables. (Ammophila arenaria Host.) Souche émettant des rhizomes longuement traçants; chaumes de 7 à 9 décimètres, droits; feuilles touffues, glauques, roulées, subpiquantes, souvent aussi lougues quele chaume ; ligule cellulovasculaire, allongée, bipartite, à lobes lancéolés, acuminés; panicule cylindrique, jaunâtre; paillettes linéaires-lancéolées, droites; paléoles munies à la base de poils trois fois plus courts qu'elles. Vivace, fleurit en juillet et août.

Sables maritimes, landes sablonneuses.

VII tribu. - stipacées.

Les stipacées présentent des locustes cylindriques ou un peu comprimées, à un fleuron fertile, disposées en une panicule rameuse ou presque simple, non digitée; la paléole externe s'enroule en cylindre autour de l'ovaire et se termine en une arête plus ou moins longue, tordue à la base; trois paléolules; stigmates sortant vers la partie inférieure ou au-dessous de la partie moyenne des paléoles.

\section{GENRE 38. - STIPE. (STIPA L.)}

Locustes uniflores, sans aucun rudiment de fleuron stérile; glume à pailletles canaliculées, acuminées ou longuement subulées, aristées, presque égales; paléoles coriaces s'enroulant en cylindre autour del'ovaire, par conséquent non carénées, à peine écartées lors de la floraison, l'externe se terminant en une arête articulée à son insertion, tordue dans sa partie inférieure, glabre ou plumeuse; paléole intérieure mulique, enveloppéc par l'extérieure; étamines renfermées dans les pa- 
léoles, d'où elles sortent à peine lors de la floraison ; anthères nues au sommet; paléolules 3 , plus ou moins épaisses, entières, soudées à leur base avec le gynophore; ovaire oblong, glabre; styles courts, soudés en une colonne inférieurement; stigmates subsessiles, plumeux; grain oblong-allongé, attenué aux deux extrémités, étroitement enveloppé par les paléoles coriaces, indurées; panicule plus ou moins rameuse.
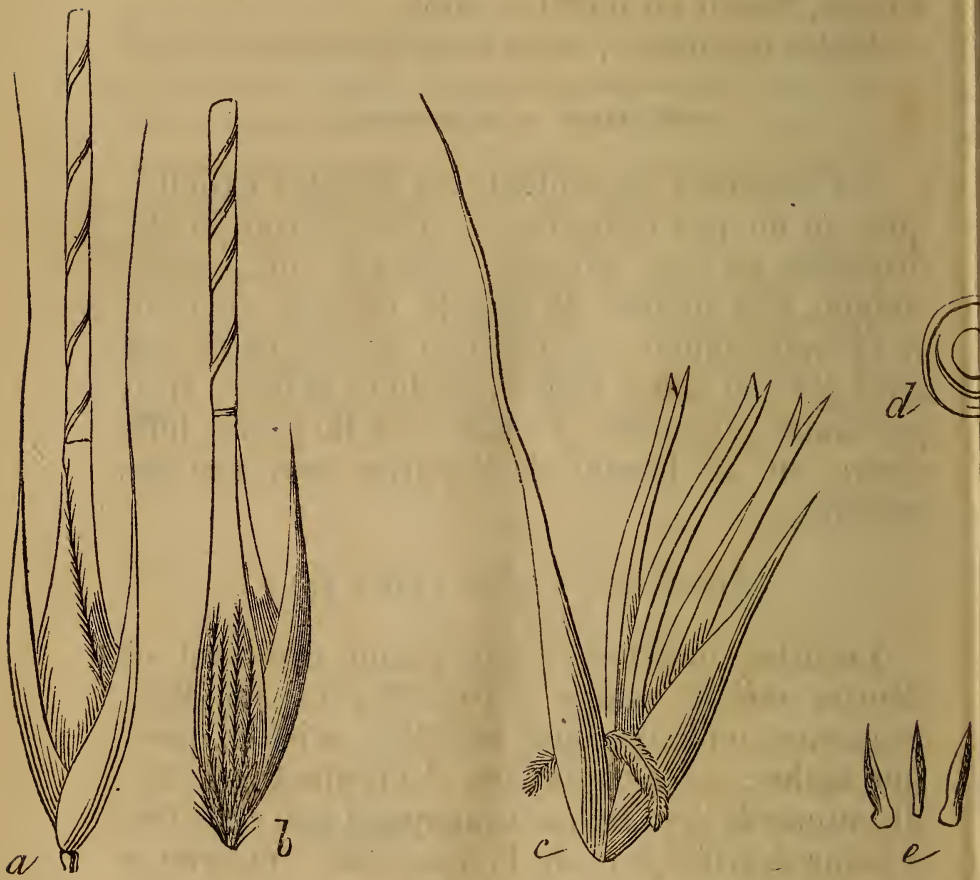

Fig. 42 (I).

(1) a Locuste de stipe empenné ; $b$-c flemron id.; $d$ coupe du fleuron faisant voir l'enroulement; $c$ glumellule à trois paléolules. 
ANALYSE DES ESPÈCES.

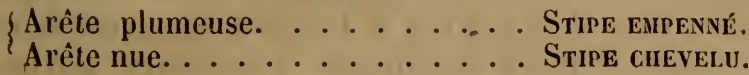

a) Stipe empenné. (Stipa pennata L.) Souche cespiteuse; chaumes de 4 à 8 décimètres, dressés; feuilles très-longues, roulées, filiformes, velues en dedans; panicule serrée, à rameaux simples ou divisés, très-souvent engainée; locustes peu nombreuses; paillettes lancéolées, aristées; paléole externe présentant à sa base cinq lignes de poils fins, soyeux, munie d'une arête longue, atteignant souvent 10 à 12 centimètres, plumeuse ou garnie de poils blancs, soyeux dans sa partie supérieure, glabre inférieurement. Vivace; fleurit en mai-juin.

Sur les coteaux arides et secs. Très-rare.

b) Stipe chevelu. (Stipa capillata L.) Souche cespiteuse ; chaumes de 7 à 18 décimètres, dressés; feuilles plus ou moins roulées ; face interne ou supérieure pubescente; ligule très-allongẻe, sourent divisée; panicule souvent engainée; locustes peu nombreuses; paléole externe portant unc arête glabre, diversement courbée, moins longue que dans l'espèce précédente. Vivace; fleurit en juinjuillet. Très-rare.

\section{GENRE 39. - LASIAGROSTIDE. (LASIAGROSTIS LNK.)}

Locustes uniflores, à fleuron courtement stipité; glume à deux paillettes membraneuses, mutiques, dépassant la locuste, l'intérietire un peu plus grande; glumelle à deux paléoles membraneuses, l'extérieure un peu comprimée latéralement, sub- 
carénée, velue-ciliée, aristée; arête non articulée, à peine tordue, subgéniculée vers le milieu; paléole interne plus courte, enroulée, binerviée. Etamines 3, à filets adnés à la base du gynophore; anthères à lobes terminaux supérieurs barbusétoilés et submucronés à la base; ovaire stipité, glabre, bifide au sommet; styles 2 , très-courts; stigmates 2 , plumeux ; paléolules 3 , membraneuses, glabres, la médiane affectant une forme différente des autres; grain fusiforme, arrondi, lisse, glabre; embryon ayant le quart de la longueur du grain; panicule rameuse, diffuse.

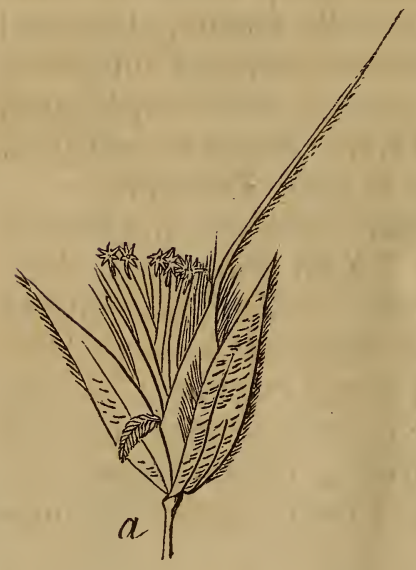

Fig. 43 (1).

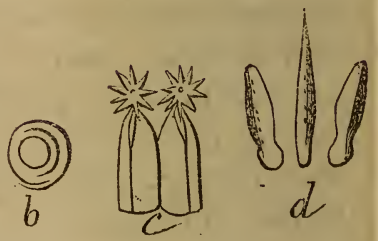

Lasiagrostide calamagrostide. (Iasiagrostis calamagrostis Wahlenb.) Souche cespiteuse-tracante; chaume rameux à la base, s'élevant souvent

(1) a Locuste de lasiagrostide calamagrostide; $b$ coupe du fleuron; c portion d'une anthère; $d$ lumellule à trois paléolules. 
d'un à deux mètres de hauteur; feuilles longues, planes ou un peu roulées sur les bords; panicule rameuse, large, longue; paillettes argentées ; paléole extérieure velue-ciliée et aristée; arête environ deux fois plus longue que la glume. Vivace; fleurit en juillet-août.

Endroits montagneux.

GeNre 40. - Piptathère. (Piptatherum P. D. B.)

Locustes uniflores; glume persistante, à deux paillettes presque égales, membraneuses, mutiques ; glumelle à deux pa!éoles subcoriaces, l'externe convexe, glabre, entourant l'interne, à peine bidentée au sommet et aristée entre les dents; arête articulée à la base, caduque; paléole interne binerviée; glumellule à trois paléolules membraneuses, glabres, la médiane plus petite, d'une autre forme; étamines 3 ; anthères nues ou barbues; ovaire stipité, glabre; styles 2 , terminaux, courts; stigmates plumeux; grain elliptique, glabre; panicule rameuse, diffuse.
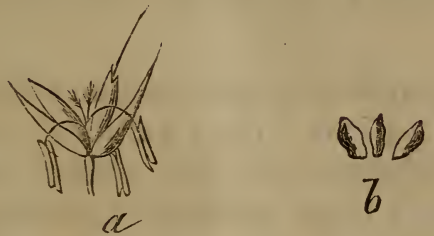

Fig. 44 (1).

Piptathère multiflore. (Piptatherum multiflorum P. D. B.) Souche cespiteuse émettant un

(1) $a$ Locuste de piptathère multiflore; $b$ paléolules; grossies. 
grand nombre de chaumes de 8 à 13 décimètres; feuilles planes, glabres; ligule allongée; panicule large effilée ou étalée, à rameaux verticillés; paléole externe à cinq nervures; paléole aristée; arête de même longueur environ qu'elle. Vivace; fleurit de mai à juillet.

Se rencontre dans les bois-taillis de quelques parties de la Flandre orientale, où elle a été semée à titre d'essai agricole.

VIII tribu. - Mtiliacées.

Les miliacées présentent des locustes un peu comprimées par le dos, disposées en panicule rameuse; les locustes contiennent un fleuron fertile; paléole externe convexe, arrondie au dos, coriace, mutique, non enroulée autour de l'ovaire; paléolules 2 ; stigmates sortant vers la partie moyenne des paléoles; grain étroitement renfermé entre les paléoles indurées.

$$
\text { GENRE 4. - MiLlet. (MLIUI L.) }
$$

Locustes uniflores, sans aucun rudiment de fleuron stérile; glume à deux paillelles convexes non carénées, mutiques, égales, glabres, ni ciliées, ni scabres sur la ligne médiane; paléoles mutiques presque égales ; étamines 5 ; paléolules 2 , épaissies, presque bifides; ovaire glabre; styles 2, courts, terminaux; stigmates plumeux ; grain ovale-oblong, atténué aux extrémités, étroitement enveloppé par les paléoles coriaces-indurées; panicule rameuse, à rameaux subverticillés. 

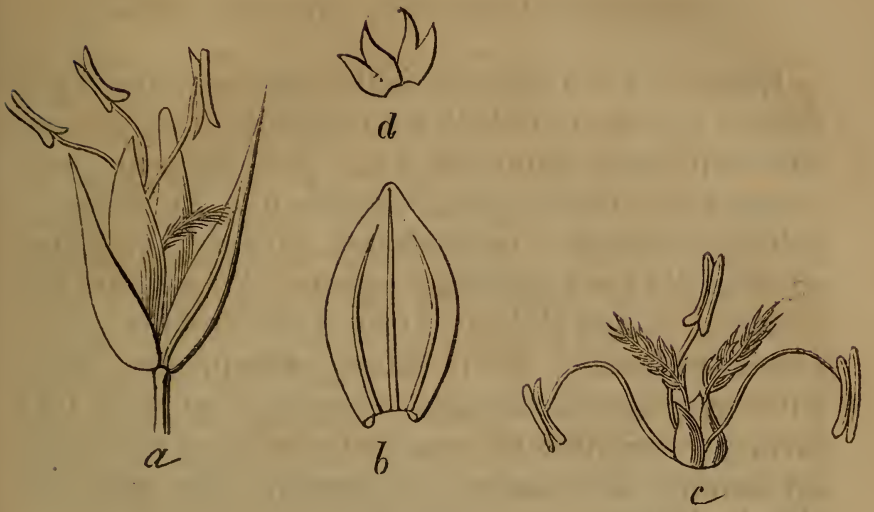

Fig. 45 (1).

Millet épars. (Milium effusum L.) Souche traçante; chaumes droits, assez forts, glabres, lisses, de 9 à 15 décimètres de hauteur; feuilles lancéolées-linéaires, glabres, lisses; ligule oblongue, obtuse, érodée; locustes disposées en une panicule ample, très-lâche et étalée, ou contractée, penchée après la floraison; paléole externe aiguë. Vivace; fleurit en mai-juin.

Dans les endroits ombragés humides.

\section{IXe tribu. - Cynodonées.}

Locustes à un fleuron fertile, disposées en épis linéaires formant, au sommet du chaume, une panicule simple, digitée; paillettes et paléoles carénées; stigmates sortant au-dessous du sommet des paléoles.

(1) a Locuste du millet épar's ; $b$ paléole externe du mẻme; $c$ organes sexucls avee les paléolules; $d$ palíolules. 
GENRE 42. - CHIENDENT. (GYNODON RIGH.)

Locustes à un fleuron fertile, accompagné d'un fleuron supérieur réduit à un pédicelle subulé ou subclaviforme; glume à deux paillettes un peu inégales et ouvertes, plus courtes que la locuste; paléole extérieure naviculaire, carénée, mutique ou apiculée au-dessous du sommet; l'intérieure bicarénée; ovaire glabre; styles 2 , terminaux; stigmates plumeux; grain oblong, comprimé, libre entre les paléoles; locustes disposées en épis filiformes, unilatéraux, partant plusieurs ensemble du sommet du chaume et formant une panicule simple digitée.
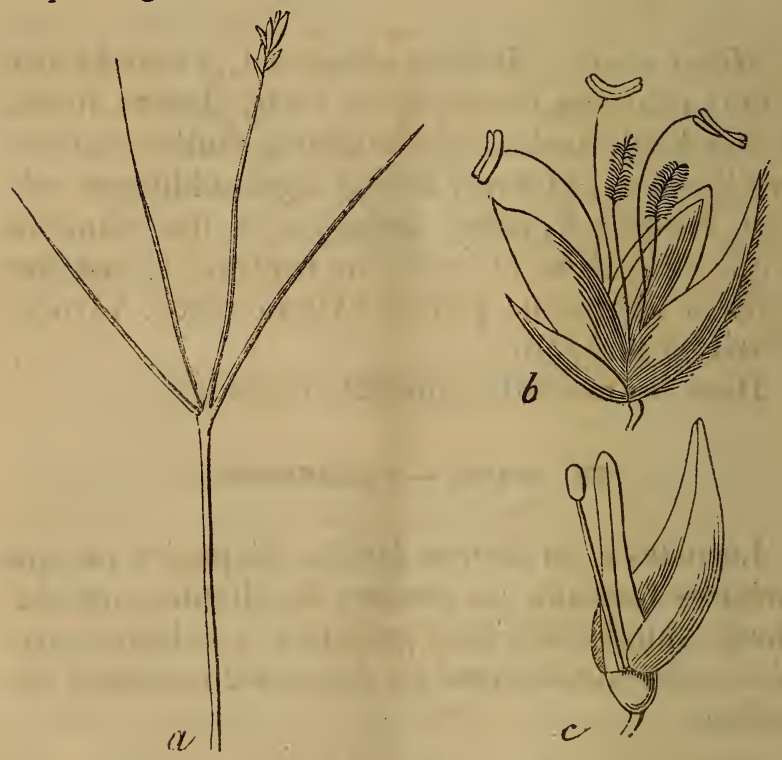

Fig. 46 (1).

(1) a Axe dinfloresence; $b$ locuste de chiendent dactyle; $c$ fleuron du mène. 
DEUXIENE PARTIE. - ANALISES ET DESCRIPTIONS. 201

Chiendent dactyle. (Cynodon dactylon. Rich.) Souche émettant des rhizomes longuement traçants, d'où s'élèvent perpendiculairement des chaumes dressés, souvent munis à leur base de bourgeons flexueux, courbés et écailleux ; feuilles courtes, placées presque sur deux rangs, glabres ou quelquefois hérissées en-dessous et garnies de longs poils à l'orifice de leur gaine; panicule simple, digitée, c'est-à-dire inflorescence composée d'un axe primitif court, se divisant en trois à seize axes secondaires qui deviennent la base d'autant d'épis linéaires, un peu rougeâtres; paléoles glabres ou un peu ciliées. Virace; fleurit de juillet à septembre.

Dans les champs en friche, dans les terres sablonneuses sujettes aux inondations, et aux bords des champs. (?)

Xe tribu. - Plactarialncées.

Les phalaridacées présentent des locustes à un fleuron fertile, disposées en épi ou en panicule spiciforme, jamais digitée, rarement étalée, à fleurons nus ou munis de deux rudiments pénicilliformes; paillettes et paléoles externes carénées, presque toujours coriaces-indurées; stigmates sortant au sommet des paléoles très-rarement inclus ou sortant vers le milieu du fleuron.

\section{INALYSE DES SOUS-TRIBUS.}

Glume nulle. ......... Asprellées. Une glume. . . . . . . . . 2.

Paléole externe mutique. . . . . Pinllaridées.

Paléole externe aristée. . . . . Alopecunées. 
ORDRE DE SUCCESSION DES SOUS-TRIBUS.

$1^{\text {re }}$ Asprellées.

2. Phalaridées

$3^{\text {e }}$ Alopécurées.

Ire sous-tribu. - A sprellées.

Locustes à un fleuron fertile ; glume nulle; paléole externe carénée; stigmates inclus ou saillants vers le milieu du fleuron (1).

GENRE 43. - ASPRELle. (ASPRELla SChreb.)

Locustes uniflores; glume nulle; glumelle à deux paléoles, l'extérieure deux fois plus large que l'intérieure, coriace, herbacée ou papyracée, comprimée, naviculaire, carénée, à 5 nervures, souvent hispide et ciliée sur le dos; l'intérieure étroite, très-comprimée latéralement, à 3 nervures; paléolules 2, petites, aiguës, presque toujours entières, glabres, rarement un peu velues; étamines 1-6 renfermées dans la paléole externe; pistil emprisonné

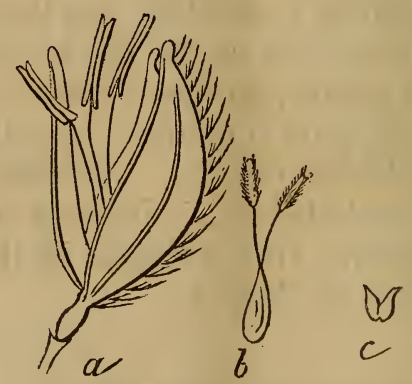

Fig. 4.7 (2).

(1) Ce dernier caractère n'a guc̀re d'importance pour la classification du genre Asprelle; comme il ne rentre dans aucune autre tribu, nous avons cru pouvoir le placer parmi les phalaridacécs, avec lesquelles il a beaucoup d'affinité. Il sera d'ailleurs facilement diagnostiqué, puisque e’est le seul gempe de graminée indigène à inflorescence paniculéc où la glume fasse défiut.

(2) a Locuste d'asprelle laux riz; $b$ pistil iul. ; $c$ palćolules id. 
en quelque sorte dans la paléole interne; ovaire turbiné; styles 2 ; stigmates 2 , plumeux, poilus; grain ovale-oblong, plus ou moins sillonné ou canaliculé, enveloppé par les paléoles, notamment par l'externe; panicule rameuse dressée, làche, libre ou engainée.

Asprelle faux riz. (Asprella oryzoïdes. Lam.) Souche à rhizomes traçants; chaumes droits ou genouillés, à nœuds relus; gaîne de la feuille supérieure un peu renflée; feuilles présentant une forte nervure scabre, assez longues, planes, trèsrudes de bas en haut, plus ou moins ciliées; ligule courte; panicule làche, étalée, souvent engainée, à rameaux flexueux; locustes subovales, subunilatérales et inférieures; paléoles ciliées sur la carène; paléolules 2, aiguës; grain finement strié, à stries longitudinales ou très-légèrement incurvées, subponctulé ou chagriné. Vivace; fleurit d'août à octobre.

Endroits marécageux, bords des fossés, des fleuves.

\section{Ire sous-tribu. - Phalaridées.}

Une glume; paléole externe mutique; stigmate sortant au sommet du fleuron.

GENRE 44. - MiBore. ( MIBORA adUIRS. )

Locustes uniflores sans rudiment de fleuron stérile; paillettes légèrement carénées, arrondies, tronquées et presque frangées, l'intérieure un peu plus courte; paléoles presque égales, pelites, minces, laciniées, soyeuses ou velues, ciliées, l'exlérieure à cinq nervures, embrassant l'intéricure qui présente deux nervures à peine marquées; les pa- 
léolules (on n'en trouve souvent qu'une) forment autour de l'ovaire une espèce de godet; ovaire glabre; styles 2, terminaux; stigmates filiformes, un peu glanduleux; grain oblong comprimé, terminé en pointe, libre entre les paléoles; épi filiforme, presque unilatéral, composé de 4-12 locustes.
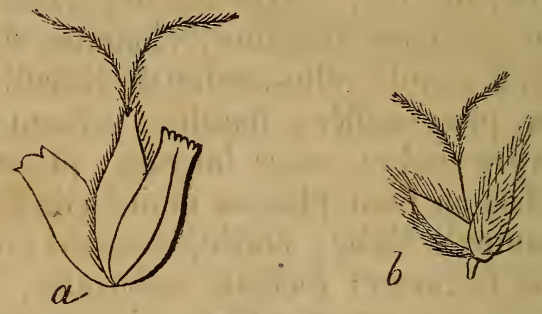

Fig. 48 (1).

Mibore naine. (Mibora minima Dmtr.) Souche cespiteuse; chaume nombreux, capillaires, feuilles courtes, étroites, n'existant qu'à la base des chaumes, qui s'élèvent à $2-4$ pouces, terminés par un épi filiforme, linéaire, verdâtre ou mêlé de violet et de pourpre, composé de 4-12 locustes subsessiles, purpurescentes. Annuelle; fleurit de mars à mai.

Dans les landes sablonneuses et dans les dunes de la Flandre occidentale.

$$
\text { GENRE 45. - ALPiste. (Phalaris L.) }
$$

Locustes à un fleuron fertile accompagné d'un ou de deux rudiments de fleurons stériles qui se présentent sous la forme d'écailles fortement ciliées simulant un corps pénicilliforme, ou glabres,

1) a Locuste de mibole naine; $b$ fleuron id. 
DEUXIÈME PARTIE. - ANALISES ET DESCRIPTIONS. 20弓

ou presque glabres et simulant des paléoles plus ou moins développées; locustes inférieures des divisions de la panicule rarement stériles; paillettes presque égales, naviculaires, carénées; à carène ailée ou non ailée, enveloppant la locuste; paléoles entières, coriaces, carénées-naviculaires, mutiques; paillettes et paléoles rarement tronquées, rongées au sommet; étamines 5 ; paléolules ovées ou lancéolées, entières, velues; ovaire glabre; styles 2 ,
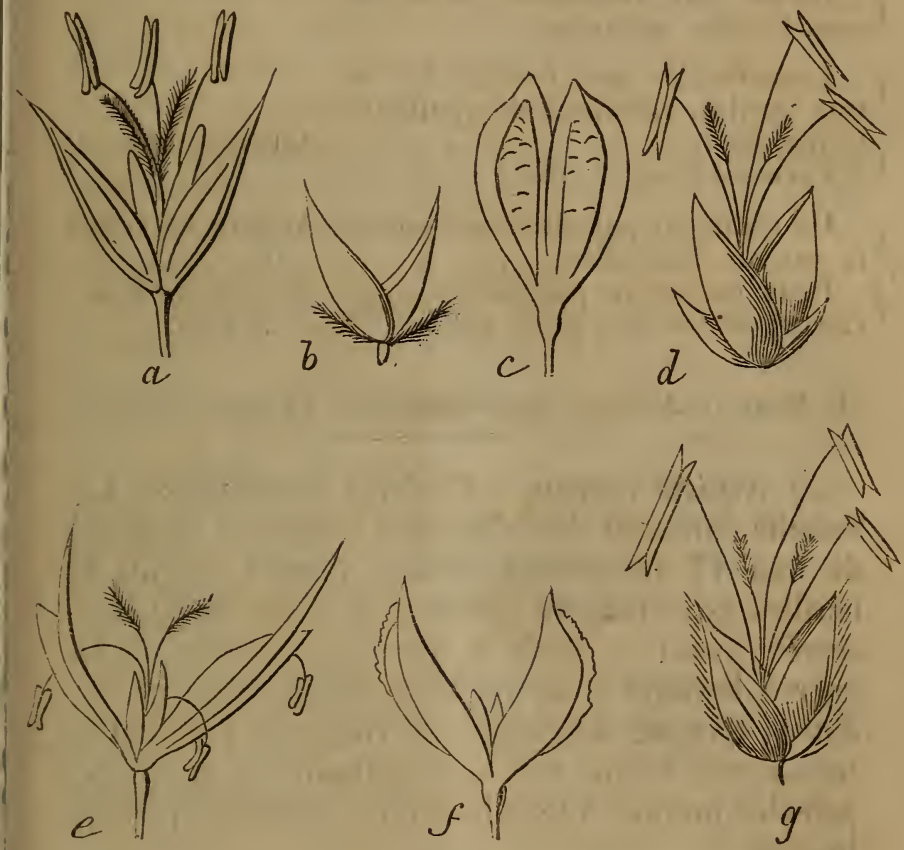

Fig. 49 (1).

(1) a Locuste d'alpiste roseau; $b$ fleuron id. arec ses rudiments étalés; $c$ glume de l'alpiste des Canaries; $d$ fleuron du mème avec ses rudiments paléoliformes; $e$ locuste de lalpiste rongée; $f$ locuste de l'alpiste aquatique; $g$ fleuron id. arec son rudiment paléoliforme. 
terminaux; stigmates longs, plumeux; grain oblong, plus ou moins comprimé, étroitement renfermé entre les glumelles; panicule spiciforme compacte ou panicule rameuse lobée.

\section{ANALYSE DES ESPÈCES.}

Deux rudiments pénicilliformes.

Plantes vivaces. . . . . . . Alpiste rosead (a)

Un ou deux rudiments paléolifor-

mes. Plantes annuelles. . . . . . 2. 2.

Locustes, les unes fertiles, les au-

$2\left\{\begin{array}{l}\text { tres stériles, réduites à des pailletles } \\ \text { tronquées. }\end{array}\right.$

tronquées. ................... Alpiste rongée (d)

Locustes toutes fertiles. . . . . . 3 .

Un rudiment paléoliforme; aile de Alpiste aquatique \{ la carène denticulée. ....... (c).

5 Deux rudiments paléoliformes, co- Alpiste des Canariaces; aile de la carène entière. . . n nies (b).

J. Deux rudiments pénicilliformes. Plantes vivaces.

a) Alpiste roseau. (Phalaris arundinacea L.) Souche émettant des rhizomes traçants; chaumes de 9 à 17 décimètres, droits, fermes, garnis de feuilles assez longues, planes ; panicule làche, res.serrée avant et après la floraison, colorée ou bigarrée de violet et de verdâtre, ou entièrement verdàtre; locustes fasciculées et entassées; paillettes très-aiguës à trois nervures saillantes, non ciliées; paléoles munies à leur base d'un rudiment pénicilliforme.

On en cultive une variété à feuilles marquées de raies longitudinales blanches et vertes. Vivace; fleurit en juin-juillet.

Sur les bords des rivières el des fossés. 
DEUXIÈie PARTIE. - ANALYSES ET DESCRIPTIONS. 207

II. Un ou deux rudiments paléoliformes. Plantes annuelles.

b) Alpiste des Canaries. (Phalaris canariensis L.) Chaumes rameux, glabres, garnis de feuilles assez larges; gaînes longues, renflées, surtout celle de la feuille supérieure; ligule plus ou moins longue et laciniée ou dentée; panicule spiciforme ovale, panachée de vert et de blanc; locustes toutes fertiles; paillettes aiguës, ailées sur le dos, à ailes entières, contenant une fleur fertile munie à la base de deux rudiments paléoliformes, glabres ou presque glabres, de moitié plus courts environ que le fleuron fertile; paléoles recouvertes de poils appliqués. Annuelle; fleurit en juillet.

Originaire des îles Canaries.

c) Alpiste aquatique. (Phalaris aquatica L.) Souche plus ou moins bulbeuse à la base; panicule spiciforme, compacte, ovale-oblongue, panachéc de vert et de blanc; locustes toutes fertiles; pailletle à carène denticulée; paléoles couvertes d'un duvet soyeux; un rudiment paléoliforme à la base du fleuron, n'atteignant pas la moitié de la longueur de celui-ci. Annuelle; fleurit en juillet-septembrc.

Cultivée.

d) Alpiste rongée. (Phalaris promorsa Lam.) Chaume droit, feuillé; feuilles à gaîne supérieure presque toujours renflée, roulée; panicule spiciforme panachée de blanc et de vert, à peine sortie de la gaîne, à rameaux un peu noueux, courbés et renflés supérieurement; partie supéricure de la paniculc composée de locustes à paillettes presque toutes entières; parlie inférieure de la panicule composée de locustes à pailleltes dont les deux tiers 
sont tronquées, caronculées; rudiments tridentésacuminés au sommet. Fleurit en juillet-août.

Annuelle, peu cultivée.

\section{GENRE 46. - FLÉOLE. (PHLEUM L.)}

Locustes à un fleuron fertile, sans rudiment de fleuron stérile, ou présentant à la base un pédicelle plus ou moins allongé; glume à paillettes presque égales, plus longues que la locuste, navi-
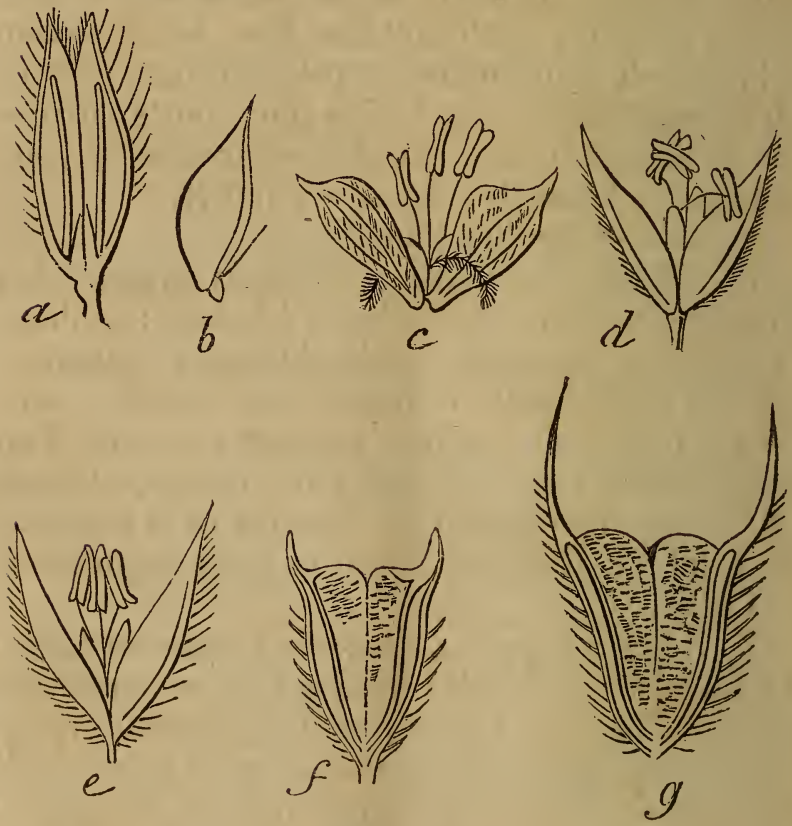

Fig. 50 (1).

(1) $a$ Locuste de fléole des sables; $b$ flcuron id. avec son rudiment pédicelliforme; $c$ locuste de fléole âpre; $d$ locuste de fléole lisse; $e$ locuste de fléole hérissée; $f$ locuste de fléole des prés; $g$ locutes de la mème à long mucron; grossis. 
culaires-carénées, plus ou moins ciliées ou scabres sur la carène, acuminées insensiblement, ou tronquées-acuminées, non soudées entreelles; paléole extérieure tronquée, mutique ou mucronée, l'intérieure bicarénée; étamines $\mathbf{5}$; paléolules lancéolées, aiguës; ovaire glabre; styles 2; stigmates en goupillon; grain oblong, légèrement comprimé, souvent terminé par deux petites pointes, libre entre la glumelle; panicule spiciforme compacte ou épi cylindrique.

ANALYSE DES ESPÈCES.

Paléole interne munie à la base d'un rudiment pédicelliforme; paillettes insensiblement acuminées. . . . .

Paléole interne dépourvue de rudiment pédicelliforme; paillettes tronquées-acuminées. ........ 5 .

Paillettes presque cunéiformes ... Fléole aPRE (b).

Paillettes non subcunéiformes. . . 3 .

Plante annuelle; panicule spici- Fléole des Sables forme, ovale-oblongue .......

Plantes vivaces; panicule spiciforme, cylindrique. . . . . . .

Paillettes ciliées sur la carène; feuilles planes assez larges, d'un vert grisâtre. . . . . . . . . . FLÉole HéRISSĖe(e)

Paillettes scabres ou ciliées sur la carène; feuilles plus ou moins enrou- FuÉole lisse. lées, étroites, d'un vert foncé sombre. (d).

Epi ovale-oblong. . . . . . . . . Fut́ole des Alpes(f) Epi cylindrique . . . . . . . . Flếle des PRÉs(e)

$\S$ I. Paillettes insensiblement acuminées; un rudiment filiforme à la base.

a) Fléole des sables. (Phleum arenarium L.) Souche cespiteuse; chaume de 1 à 3 décimètres, 
venant en touffes; gaines des feuilles supérieures un peu renflées; panicule spiciforme, ovale-oblongue, atténuée; paillettes lancéolées à carènes ciliées. Aunuelle; fleurit en juin-juillet.

Fréquente dans les sables maritimes, les dunes.

b) Fléole âpre. (Phleum asperum Vill et Jacq.) Chaumes venant en touffes, rameux à la base; feuilles glabres; panicule spiciforme ou épi cylindrique, ordinairement vert, rude au toucher; paillettes presque cunéiformes, un peu gibbeuses, glabres, non ciliées, mucronées. Annuelle; fleurit en juin-juillet.

Dans les endroits secs.

c) Fléole hérissée. (Phleum hirsutum Sut.) Souche cespiteuse; chaume de 5 à 9 décimètres, dur, glabre, strié, couché et feuillé dans le bas, nu et redressé dans le haut; feuilles planes, assez larges, d'un vert grisâtre; panicule spiciforme, cylindrique, continue, ayant 11-15 millimètres de diamètre à la floraison; paillettes lancéolées, entières, ciliées sur le dos. Vivace; fleurit en juinjuillet.

Dans les prés sces.

d) Fléole lisse. (Phleum love Schrad.) Souche cespiteuse; chaume de 5 à 9 décimètres, glabre, rude, un peu genouillé dans le bas; feuilles plus ou moins enroulées, étroites, pointues, d'un vert foncé sombre et scarieuses sur les bords; les supérieures fort courtes; ligule très-courte, tronquée; panicule spiciforme, cylindrique, grêle, souvent un peu interrompue, ayant 8.9 millimètres de diamètre à la floraison ; pailletles à carène scabre ou cilice. Vivace; fleurit en mai el en juin.

Dans les lieux arides, bords des bois et des prés secs. 
§ II. - Pailletles tronquées-acuminées.

e) Fléole des prés. (Phleum pratense L.) Souche cespiteuse; chaumes de 7 à 15 décimètres, droits ou un peu coudés inférieurement, présentant parfois une espèce de rhizome alternativement étranglé et renflé; feuilles assez larges, planes; épi verdàtre, cylindrique, serré, obtus; paillettes oblongues, tronquées transrersalement et surmontées d'un pointe aiguë plus courte que la paillette; carène verte fortement ciliée.

Celte espèce présente quelques variétés remarçuables, dont l'une a le rhizome continu, non alternatirement étrangléet renflé; l'autre présente un chaume bulbeux à la base ou un rhizome alternativement étranglé et renflé. Vivace; fleurit de mai à septembre.

Dans les prairies et aux bords des chemins.

f) Fléole des Alpes. (Phleum alpinum L.) Souche cespiteuse; chaume de 2 à 4 décimètres, droit ; feuilles courtes ; épi court, ovale-oblong, ordinairement d'une teinte violette; paillettes oblongues-tronquées, munies d'une pointe ou soie aristiforme, dont quelques-unes au moins plus longues qu'elles, subvelues; carène ciliće. Vivace; fleurit de juin à aoùt.

Dans les lieux herbeux, élevés, ainsi que dans les terrains sablonneux et maritimes. (?)

H1 sous-tribu. - Alopécurées.

Une glume; glumelle réduite à une paléole externe munie d'une arête; stigmates sortant au sommet du fleuron.

geNRE 47. - VULPin. (Alofecunus L.)

Locustes à une fleur fertile dépourvue de rudi- 
ment de fleuron stérile; glumes à deux paillettes égales, naviculaires, soudées dans leur partie inférieure : cette soudure se prolonge quelquefois jusque vers le milieu de leur longueur; glumelle à une seule paléole comprimée, soudée en cornet dans sa partie inférieure, et portant une arête dorsale ou épibasilaire; étamines 5 ; ovaire surmonté

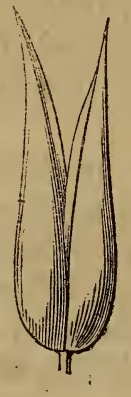

$a$

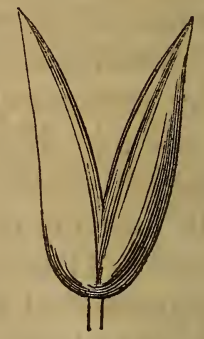

$b$
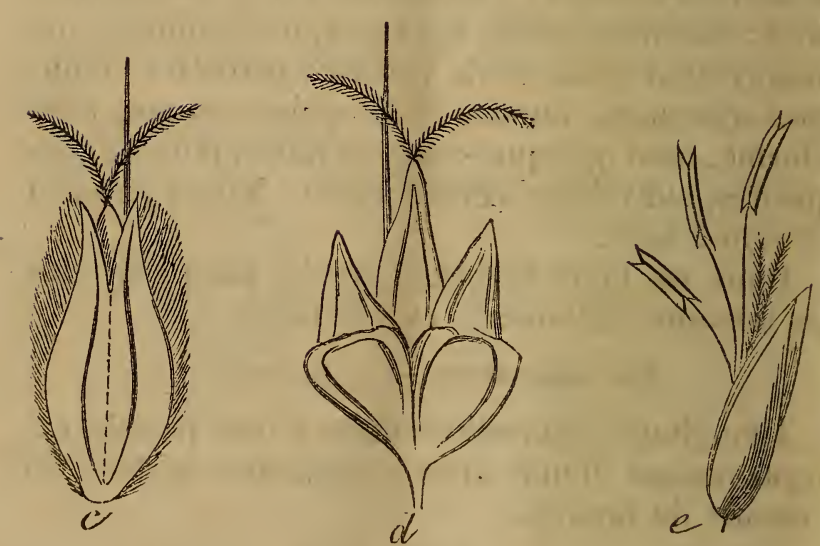

Fig. 51 (1).

(1) a et $b$ Glumes; $c$ locuste de vulpin des champs; $d$ locuste de vulpin utriculé; $e$ fleuron de vulpin où l'on a dégagé les stigmates. 
de 2 styles terminaux, soudés en une colonne; stigmates 2, filiformes; grain oblong, libre entre la glumelle; panicule spiciforme compacte, ou épi plus ou moins cylindrique, atténué aux deux extrémités, rarement ovale.

\section{ANALYSE DES ESPÈCES.}

Paillettes soudées dans leur tiers inférieur...............

Pailletles soudées à la base seulement............. 4 .

Rameaux de la panicule portant 4-6 locustes; plante vivace ...... VulpIN DES PRÉS (a)

Rameaux de la panicule ou de l'épi ne portant qu'une ou deux locustes; plante annuelle........

Epi ovoïde ou ovoïde-oblong; gaine de la feuille supérieure renflée. . . Vu Vupin utriculé(c) $\left\{\begin{array}{r}\text { Epi cylindrique allongé, attenuéaux Vulpin } \\ \text { deux extrémités ....... (b). }\end{array}\right.$

Chaume bulbeux à la base ...... Vulpin Bulbeux (d) Chaume non bulbeux à la base. . . 5 .

Arête s'insérant vers le quart inférieur ou vers la base de la paléole et dépassant longuement la glume .. Vulpingenouillé (e) Arête s'insérant vers le milieu de la longueur de la paléole et dépassant à peine ou ne dépassant pas la glume. Vulpin fauve (f).

§I. Paillettes soudées dans leur tiers inférieur.

a) Vulpin des prés. (Alopecurus pratensis $\mathbf{L}$.) Souche cespiteuse; chaumes de 7 à 10 J̀ décimètres, glabres; feuilles glabres, rudes sur les bords, aiguës; gaine de la feuille supérieure renflée; rameaux de la panicule spiciforme courts, portant quatre à six locustes; paillettes aiguës, blanchâtres, velues et ciliées sur le dos, soudées dans leur tiers inférieur ; paléole g̣labre, portant une arête 
presque basilaire. Cette espèce a quelques variétés qui dépendent du sol sur lequel elles se développent; on distingue notamment les suivantes :

$1^{0}$ Le Vulpin des prés pàle. Chaume et feuilles d'un vert pale; gaine quatre fois plus longue que la lame; arète plus longue que la glume.

$2^{\circ}$ Le Vulpin des prés aquatiques. Chaume de dix à dix-sept décimètres, glabres, ainsi que les feuilles qui sont rudes; gaine scabre vers le haut; panicule ayant sept à douze centimètres de longueur; arète plus longue que la glume.

$5^{\circ}$ Le Vulpin des prés tondu. Arète plus courte que la glume ou la dépassant à peine.

Vivace; fleurit de mai à septembre.

Dans les prés humides, sur les bords des fossés et dans les bois frais.

b) Vulpin des champs. (Alopecurus agrestis L.) Chaumes de quatre à huit décimètres, droits ou couchés, croissant en touffes; feuilles assez larges à gaines cylindriques; épi cylindrique, longy, atténué aux deux extrémités; rameaux portant 1-2 locustes; paillettes soudées dans leur moitié inférieure, aiguës, glabres, vertes ou panachées de rouge et de vert; carène légèrement ailée et eiliće; paléole externe munie d'une arète très-longue, plus ou moins tortillée. Cette espèce présente deux variétés : dans l'une les anthères sont violettes, dans autre elles sont d'un blane jaunitre.

Dans les moissons, les champs incultes et sur les bords des chemins.

c) Vulpin utricule. (Alopecurus utriculatus Pers.) Chaumes de deux ì trois décimètres, droits ou ascendants, s'élevant d'une toufie de feuilles; feuilles molles; gaine supérieure renflée, ventrue et vésiculeuse; panicule spiciforme, compacte, 
courte, ovale; rameaux portant une ou deux locustes; paillettes renflées, arquées et ailées, surmontées d'une pointe aiguë, verte et comprimée; arète tordue, longue et divergente. Annuel; fleurit en mai-juin.

Dans les prairies humides. (?)

II. Paillettes libres ou soudées seulement à la base.

d) Vulpin Gulbeux. (Alopecurus bulbosus L.) Souche cespiteuse; chaumes de trois à huit décimètres, grêles, droits, bulbeux à la base; paléoles glabres, étroites, pointues; épi grêle, très-simple, un peu velu, cylindrique, atténué aux extrénitéé, d'un vert pâle; arêtes longues, géniculées. Vivace; fleurit en juin-juillet.

Prés humides salins.

e) Vulpin genouillé. (Alopecurus geniculatus L.) Souche cespitcuse; chaumes de quatre à treize décimètres, couchés, coudés aux nouds inférieur's, souvent radicants à la base, glabres; feuilles à gaines un peu comprimées; panicule spiciforme cylindrique, jamais simple, mais plus ou moins rameuse; paillettes obtuses, ciliées; paléole munie d'une arête insérée vers la base ou vers le quart inférieur de sa longueur et deux plus fois longue qu'elle; anthères brunes après la floraison. Vivace; fleurit de juin à août.

Dans les endroits herbeux, humides, marécaeageux et tourbeux.

f) Vulpin fauve. (Alopecurus fulvus Smith.) Souche cespiteuse; chaumes de quatre à neuf décimètres, couchés et coudés aux nouds inférieurs, puis ascendants, glabres; gaines d'un vert glauque et blanchatres; panicule spiciforme, cylindrique, 
un peu amincie aux extrémités; paillettes obtuses, ciliées; paléole munie d'une arête aussi longue qu'elle environ, insérée vers le milieu de sa hauteur; anthères jaune-safran après la floraison. Vivace; fleurit de mai à août.

Dans les endroits herbeux, humides, aux bords des fossés; peu commun dans les prairies inondées pendant l'hiver.

\section{GENRE 48. - POLYPOGON. (POLYPOGON DERF.)}

Locustes à un fleuron fertile; glume à deux paillettes presque égales, légèrement échancrées et surmontées chacune d'une arète; paléoles membrancuses, l'externe munie d'une arête courte, presque terminale; l'interne bifide, dentée; étamines 3 ; ovaire turbiné; style profondément

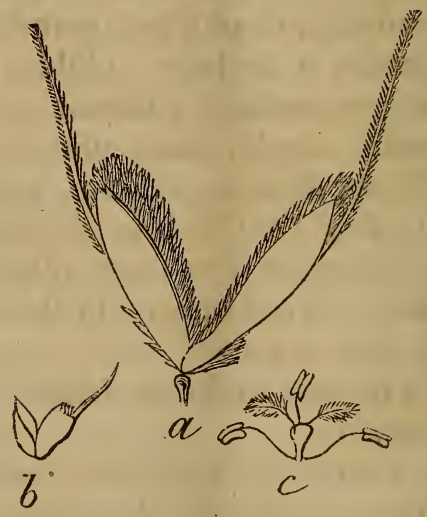

Fig. 32 (1).

1) a Glume du polypogon de Montpellier; $b$ glumelle du mêine; e organes sexucls iil., tous grossis. On en a tronqué les arèles. 
DEUXIĖME PARTIE. - ANALYSES ET DESCRIPTIONS. 217

divisé ; stigmates 2, plumeux; grain peu adhérent aux paléoles et non sillonné; panicule spiciforme plus ou moins compacte.

ANALYSE DES ESPÈCES.

(Souche cespiteuse, annuelle; paillettes munies d'une arête trois fois plus longue qu'elles. ........ Polypogon DE

Souche traçante, vivace ; arêtes de Montpellier. (a) même longueur environ que les pail- Polypogon des lettes.............. nivages. (b)

a) Polypogon de Montpellier. (Polypogon monspelliensis Desf.) Chaume de 5 à 8 décimètres, un peu flexueux, glabre; feuille supérieure à gaine membraneuse; panicule d'un vert pàle, virant vers le jaune, resserrée en épi compacte, quelquefois divisée en paquets ou lobes inégaux; paillettes pubescentes, scabres ou ciliées, portant une arète trois fois aussi longue qu'elles; paléoles externes aristées au-dessous du sommet. Cette espèce présente trois variétés distincles qui sont caractérisées :

La première, par sa panicule spiciforme lobée; la deuxième, par sa panicule spiciforme compacte et par ses paillettes entières ou légèrement fendues, et la troisième, par sa panicule spiciforme compacte et par ses paillettes fendues jusqu'au milieu. Annuel ; fleurit en juillet.

Il se trouve dans les sables marilimes.

b) Polypogon des rivages. (Polypogon littoralis Smith.) Souche traçante; chaumes de 5 à 7 décimètres, glabres; feuilles supérieures à gaines plus ou moins herbacées ou membrancuses; panicule 
spiciforme; arêtes des paillettes ne dépassant pas ou ne dépassant guère celles-ci en longueur ; paléoles externes munies d'une arête presque terminale. Vivace; fleurit en juillet et août.

On dit qu'il se trouve dans les mêmes licux que le précédent. (?)

\section{XI tribu. - Panicacées.}

Les panicacées présentent des locustes à un fleuron fertile, disposées $1^{\circ}$ en panicule simple digitée, à digitations au nombre de 3-14, rapprochées au sommet du chaume, $2^{\circ}$ en panicule simple à axes secondaires alternant sur l'axe primaire, ou $3^{\circ}$ en une panicule rameuse élalée; les paillelles et paléoles externes sont à dos convexe ou plan, non caréné, et ne s'enroulent point autour de l'ovaire; lesstyles sont allongés et les stigmates sortent vers le sommet des glumelles.

ANALYSE DES SOUS-TRIBUS.

Locustes géminées, l'une fertile, 1 hermaphrodite, l'autre stérile ou mâle. AndropogonéEs.

Locustes toutes fertiles ......

2.

Locuste à un fleuron fertile dépourvu de rudiment de fleuron stérile panicule non digitée. ......

2 Locuste à un fleuron fertile accompagné d'un ou de plusieurs rudiments paléoliformes ou panicule simple digitée............... Panictes.

3 Paléole externe aristée. . . . . Gastridićes.

Palćole externe mutique. . . . . Traginées. 
DEUXIĖME PARTIE. - ANALYSES ET DESCRIPTIONS. 219

ORDRE DE SUCCESSION DES SOUS-TRIBUS.

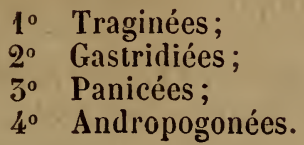

Ire sous-tribu. - Traginées.

Locustes à un fleuron fertile dépourvu de rudiment de fleuron stérile; paléole externe mutique ; panicule non digitée.

\section{GENRE 49. - TRAGIER. (TRAGUS DESF.)}

Locustes uniflores; paillette inférieure (intérieure, parce qu'elle répond à la face plane de la locuste) très-petite, plane, mince, membraneuse; la supérieure beaucoup plus grande, à dos convexe, coriace, presque cartilagineuse, à sept nervures chargées d'aspérités crochues, enveloppant la locuste; paléoles membraneuses, concaves, mutiques, très-inégales; étamines 3 ; paléolules 2 ; ovaire glabre; styles 2 , terminaux, divergents ; stigmates 2, plumeux; grain oblong, plus ou moins

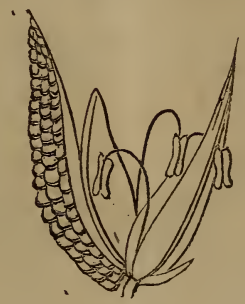

Fig. อ5 (1).

(1) Locuste de tragier en grappes. 
comprimé, libre entre les paléoles ; locustes géminées, ternées, quaternées ou quinées, disposées en une panicule simple; locustes supérieures ordinairement stériles.

Tragier en grappes. (Tragus racemosus Desf.) Chaumes rameux, étalés, redressés ; feuilles courtes, un peu ciliées; locustes médiocrement pédonculées, disposées en une panicule simple, grêle, lâche, linéaire, d'une teinte purpurine ou rougeâtre à sa maturité. Annuel; fleurit en juin.

Se trouve dans les terrains secs et sablonneux, le long des côtes maritimes. (?)

\section{Ire sous-tribu. - Gastridiées.}

Locustes à un fleuron fertile dépourvu de rudiment d'un deuxième fleuron; paléole externe aristée; panicule non digitée.

genRe 50. - Gastridier. (Gastridium. P. D. B.)

Locustes uniflores; glume à deux paillettes convexes-ventrues à la base, environ trois fois plus

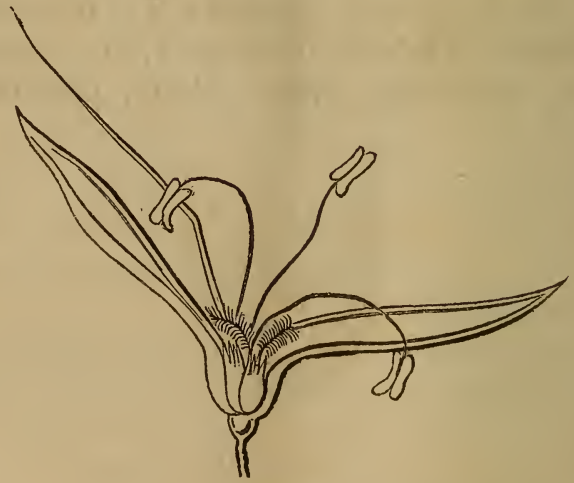

Fig. 54 (1).

(1) Locuste de gastridier leudigère. 
longues que la glumelle qui est à deux paléoles inégales, coriaces; paléole externe à 5 - 5 dents au sommet, munie d'une arête subapicale; l'interne échancrée; paléolules 2, submembrancuses ou à peine charnues, presque fendues ; ovaire ovale-lurbiné; styles 2 , très-courts ou soudés en un seul, terminaux; grain oblong, un peu comprimé, aminciaux extrémités el làchement enveloppé par les paléoles.

Gastridie lendigère. (Gastridium lendigerum P. D. B.) Souche cespileuse; chaumes dressés, rameux, feuillés; feuilles inférieures souvent roulées; les caulinaires planes, pointues ; panicule quelquefois engainée, d'un vert tendre, soyeuse; locustes dressées, plus ou moins serrées, d'un vert pâle ; paillettes très-aiguës, longues ; glumelle courte; paléole externe à trois ou quatre dents; arète fine, presque toujours genouillée. Annuelle; fleurit de mai à juillet.

Indiquée sur les bords de la Meuse. (?)

\section{III sous-tribu. - Panicées.}

Locustes à un fleuron fertile accompagné de restiges d'un ou de deux fleurons stériles, rarement nuls par avortement.

$$
\text { GENRE 51. - SÉtAiRE. (SETARIA B. D. B.) }
$$

Locustes à une fleur fertile accompagnée d'un fleuron inférieur stérile, réduit à deux paléoles inégales, ou à une paléole simulant une troisième paillette; glume à deux paillettes, l'inférieure (in(érieure) très-petite, la supérieure plus grande, convexe, mutique; paléoles convexes, indurées; l'inférieure mutique embrassant la supérieure; ovaire glabre; styles 2 , terminaux; stigmates plu- 
meux; grain ovoïde comprimé, plan d'un côté, convexe de l'autre, renfermé entre les paléoles; locustes présentant à la base une ou plusieurs soies roides, denticulées, scabres, rudiments d'autant de locustes réduites à leur pédoncule, disposées en une panicule spiciforme, presque simple ou composée, lobée, compacte, quelquefois interrompue.
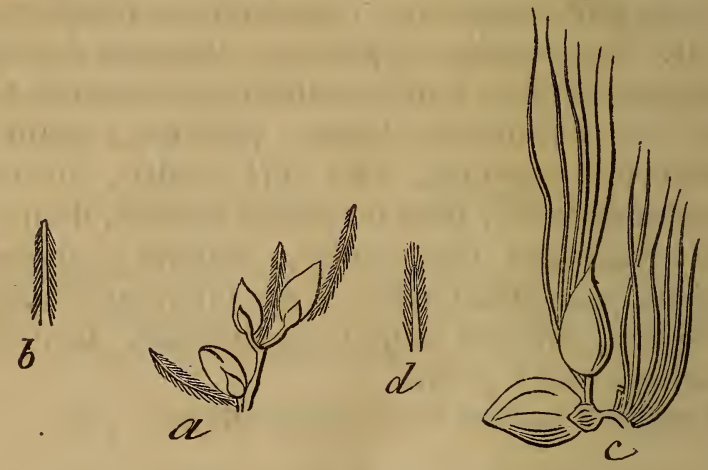

Fig. (54).

ANALYSE DES ESPÈCES.

Soies de l'involucre à denticules 1 dirigées de bas en haut. . . . ...

2.

1 Soies de l'iurolucre à denticules Sétaire verticildirigées de haut en bas. ...... LÉE (a).

(Paléole externe du fleuron fertile, Sétarre glauque $2\left\{\begin{array}{l}\text { rugucuse transversalement. . . } \\ \text { Paléole externe du flenron fertile, }\end{array}\right.$ lisse ou presque lisse transversalement.............

5.

(1) $a$ Ramean fleuri de la sétaire verticillée; $b$ portion de soie faisant voir la direction des denticules de haut en bas; $c$ rameau fleuri de la sétaire verte ; $d$ portion de soie faisant voir la direction des denticules de bas en haut. 
DEUXIĖME PARTIE. - ANALYSES ET DESCRIPTIONS. 223

Involucre unilatéral de 1 -2 soies pour chaque locuste; panicule spiciforme ovale-oblongue, à axes secon- Sétaire d'Italie daires allongés. ....... (d).

Involucre de 4-16 soies pour une, deux ou plusieurs locustes; panicule spiciforme, cylindrique, à axes secondaires courts ......... Sétaire verte(c).

a) Sétaire verticillée. (Setaria verticillata. P. D. B.) Chaumes de $\breve{5}$ à 15 décimètres, plus ou moins droits et rameux à la base, rudes au-dessous de l'axe fleuri; feuilles relues sur les bords de la gaine ; ligule mixte; locustes disposées en petits paquets verticillés formant unc panicule spiciforme serrée, quelquefois interrompue, cylindrique, rerdâtre et souvent un peu violette ; involucre composé de soies à denticules, la plupart dirigées de haut en bas, ce qui les rend accrochantes de bas en haut ; paléoles lisses. Annuelle; fleurit en juin-aoùt.

Dans les champs et les jardins, aux bords des chemins.

b) Sétaire glauque. (Setaria glauca P. D. B.) Chaume de 4 à 9 décimètres, simple ou rameux ; feuilles un peu glauques, munies de quelques poils sur la face supérieure; ligule mixte; locustes subsessiles disposées en panicule spiciforme, simple, cylindrique, resserrée ; involucre ordinairement à 8-10 soies denticulées, presque toutes dirigées de bas en haut, roussâtres; paléoles du fleuron fertile rugueuses transversalement.

Il en est une variété à chaume aplati couché sur le sol. Annuelle; fleurit de juillet à septembre.

Dans les champs sablonneux.

c) Sétaire verte. (Setaria viridis P. D. B.) Touffes composées de chaumes de 5 à 7 décimè- 
tres, rudes au-dessous de l'axe fleuri; feuilles larges, velues vers les bords de leur gaine: locustes disposees par petits paquets subsessiles formant une panicule spiciforme, cylindrique, verdâtre, serrée, droite; involucre pour deux ou plusieurs locustes, ordinairement à 4-6 soies, à denticules dirigées presque toutes de bas en haut, ce qui les rend accrochantes de haut en bas; paléoles du fleuron stérile presque lisses.

Il en est une variété plus grande dans toutes ses parties, que M. Dumortier considère comme une espèce et qu'il appelle Sétaire à beaucoup de soies (setaria multiseta). Annuelle ; fleurit en juilletaoût.

d) Sétaire d'Italie. (Setaria italica P. D. B.) Chaumes de 7 à 13 décimètres, droits, garnis de feuilles larges présentant quelques poils vers leur base, et souvent un peu ondulées, velues vers et sur les bors de la gaine; ligule mixte; locustes insérées sur l'un des côtés des axes secondaires ou tertiaires plus ou moins allongés, formant des glomerules subunilatéraux, disposées en panicule spiciforme, ovale-oblongue, compacte, lobée, souvent interrompue à la base; axe couvert de poils laineux, penché à la maturité; involucre unilatérale à 1-2 soies pour chaque locuste, à denticules dirigées de bas en haut, par conséquent, accrochantes de haut en bas; paléoles lisses. On en cultive plusieurs variétés dont les principales sont:

$1^{\circ}$ La sétaire. d'Italie maritime. (Setaria italica maritima Mich. et Lej.) Soies de l'involucre rougeâtres ou purpurines, trois fois au moins plus longues que la locuste.

$2^{\circ}$ La sétaire séteuse. (Setaria setosa $\mathrm{R}$ et $\mathrm{S}$.) Soies de i'involucre très-longues, jaunâtres. 
DEUXIEME PARTIE. - INALYSES ET DESCRIPTIONS. 22\%

$3^{\circ}$ La sétaire de Germanie. (Setaria gormanica.) Panicule contractée, jaunàtre; soies de l'involucie plus courtes, rarement plus longues que les locustes.

GENRE 52. - Digitaine. (Digitaria SCOP.)

Locustes à un fleuron fertile accompagné d'un fleuron stérile réduit à une paléole à $4-7$ nervures; glumes à deux paillettes, l'inférieure souvent très-petite, presque imperceptible ou nulle et

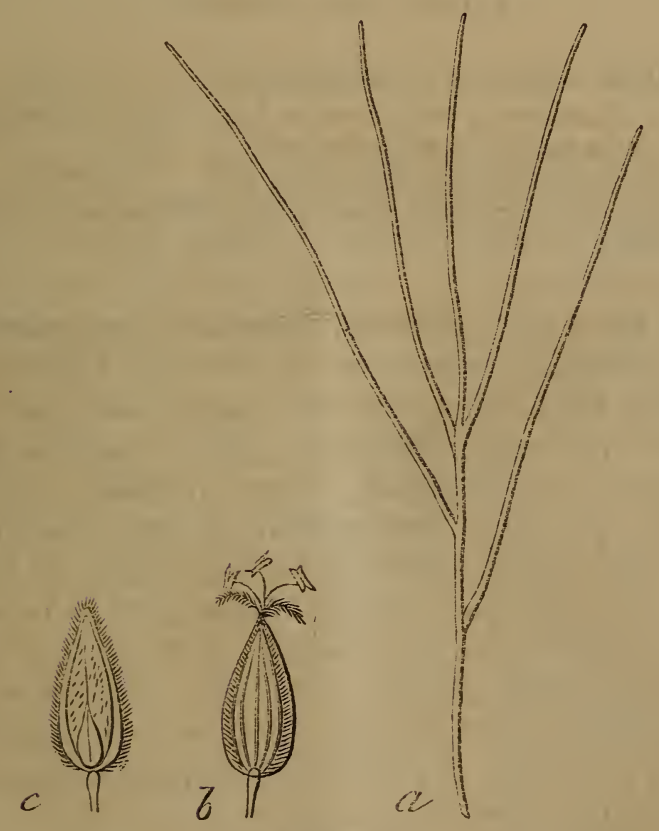

Fig. $56(1)$.

(1) a Axe dinflorescence; $b$ locuste vue par la face convexe ou externe; $c$ locuste vue par la face interne ou plane. 
anerviée; la supérieure plus grande; paléoles coriáces, mutiques, l'extérieure enveloppant l'intérieure au moins sur ses bords; ovaire glabre; styles 2, terminaux; stigmates plumeux; grain oblonǵ, arrondi, convexe sur les deux faces, enveloppé par la glumelle; axe primaire de l'inflorescence se divisant en 2-12 axes secondaires qui deviennent la base d'autant d'épis linéaires, unilatéraux et formant une panicule simple digitée ou ailée; locustes ordinairement géminées.

\section{ANALYSE DES ESPÈCES.}

$\left\{\begin{array}{l}\text { Paillette supérieure atteignant en- } \\ \text { viron le tiers ou la moitié de la lon- Digitaire sanguine } \\ \text { gueur de la paléole correspondante. } \\ \text { Paillette supérieure atteignant en- } \\ \text { viron la longueur de la paléole corres- Iigrtatre glabre } \\ \text { pondante.............. }\end{array}\right.$

a) Digitaire sanguine. (Digitaria sanguinalis Scop.) Souche cespiteuse; chaumes de 3 à 9 décimètres, droits ou ascendants ; feuilles assez larges, molles, souvent rougeàtres, plus ou moins poilues, ainsi que les gaines, rarement glabres; ligule courte; panicule simple à 5-10 épis linéaires, dont souvent trois placés à l'extrême bout de l'axe primaire de l'inflorescence, ordinairement colorés de pourpre; locustes oblongues-lancéolées; glume à paillettes très-inégales entre elles, glabres ou à peine pubescentes sur les nervures, ou cilićesvelues; pailletle supérieure alleignant environ le tiers ou la moitié de la longueur de la paléole correspondante, l'autre presque toujours nulle. Annuelle; fleurit tout l'été.

Dans les terressablonneuses cultivées el incultes. 
DEUAIÈME PIRTIE. - ANALYSES ET DESCRIPTIONS. 227

b) Digitaire glabre. (Digitaria glabra P. D. B., non Dmtr.) Souche cespiteuse; chaumes de 5 à 6 décimètres, étalés; feuilles et gaînes glabres, ligule courte; axe primaire de l'inflorescence se divisant ordinairement en 2-4 axes secondaires, souvent d'un rouge brun; locustes ovales-oblongues; paillette supérieure atteignant environ la longueur des paléoles, glabre ou à peine ciliée sur les nervures, l'autre presque toujours nulle. Annuelle; fleurit de juillet à septembre.

Cette espèce se rencontre plus fréquemment que la précédente.

GENRE 53. - OPLISHÈNE. (OPLISMENUS DMTR.)

Locustes à une fleur fertile accompagnée d'un fleuron inférieur stérile, à paléoles très-inégales, dont la plus grande, souvent aristée, simule une troisième pailıtte; glume à deux paillettes, dont l'inférieure est beaucoup plus petite que la supérieure ; paléoles coriaces, presque égales, l'extérieure terminée par une pointe plus ou moins allongée et embrassant l'intérieure; élamines $\mathbf{5}$; ovaire glabre; styles 2, terminaux; stigmates plumeux; grain oblong, convexe, non adhérent aux glumelles; locustes dépourvues de soies denticulées à la base; paillelles plus ou moins hérissées de poils et d'aspérités; panicule simple, composée de divisions presque alternes, parfois conjuguées sur l'axe primaire de l'inflorescence, très-rarement subrameuse. 


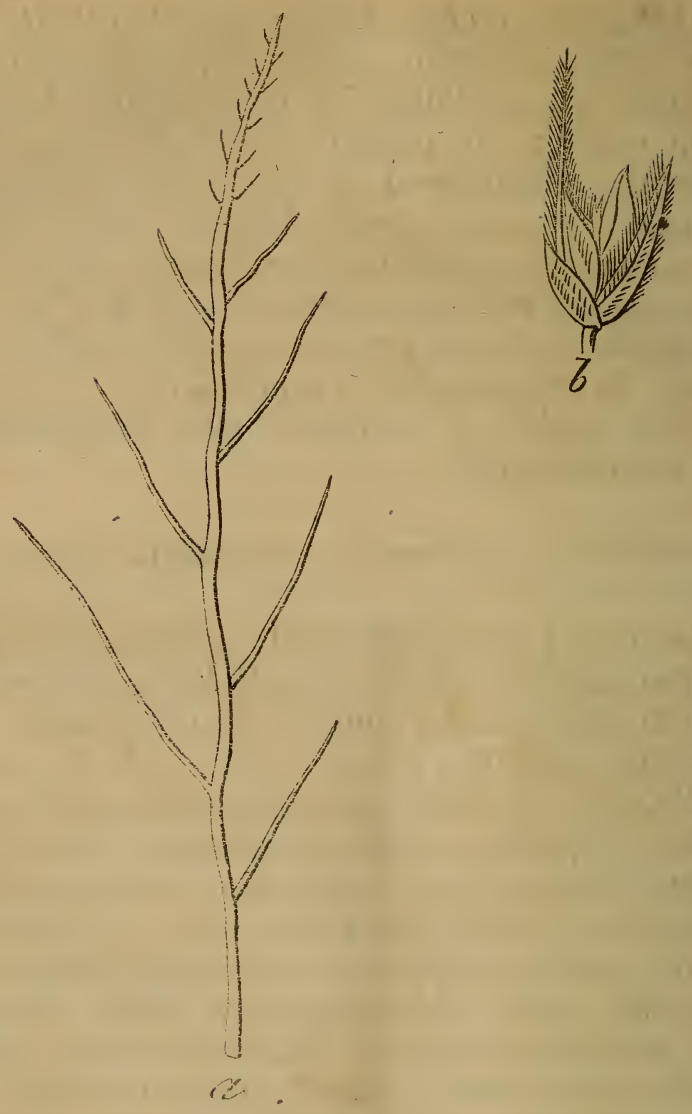

Fig. 57 (1).

Oplismène pied de coq. (Oplismenus crus galli. Dmtr.) Chaumes de 4 à 8 décimètres, droits ou plus ou moins couchés; feuilles larges, planes, glabres, ordinairement ondulées sur les bords;

1) a . Ixe re ل̈̈nllorescence; b locuste doplismèue pied de cor. 
ligule nulle, remplacée par un demi-cercle couleur de rouille ou blanchâtre; panicule simple ou axe primaire allongé, présentant des axes secondaires disposés alternativement, d'autant plus courts qu'ils approchent du sommet, et formant la base de petits faux épis presque unilatéraux, rarement rameux; paillettes striées, hérissées de poils et d'aspérités; paléoles mutiques ou terminées par une pointe plus ou moins allongée.

Cette espèce présente trois variétés assez distinctes qui sont :

$1^{\circ}$ Le panis pied de coq ordinaire. Paléoles courtement aristées ou mutiques et axe à cing angles.

$2^{\circ}$ Le panis pied de coq aristé. Paléoles longuement aristées ; axe à cinq angles.

$5^{\circ}$ Le panis pied de corbeau. Axe à 2-ā angles. Annuel; fleurit en juillet et en aoùt.

Champs sablonneux, aux bords des fossés et des chemins.

\section{GENRE 54. - PANIS. (PANICUM L.)}

Locustes à un fleuron fertile accompagné d'un fleuron neutre réduit à une ou deux paléoles; glume à deux paillettes, l'inférieure plus petite que la supérieure; paléoles du fieuron mutiques, prèsque égales, minces, membraneuses; étamines 3 ; paléolules 2, plus courtes que l'ovaire; oraire glabre; styles 2; stigmates 2, plumeux; grain enveloppé par la glumelle dure, coriace; locustes disposées en une panicule rameuse plus ou moins étalée ou penchée. 


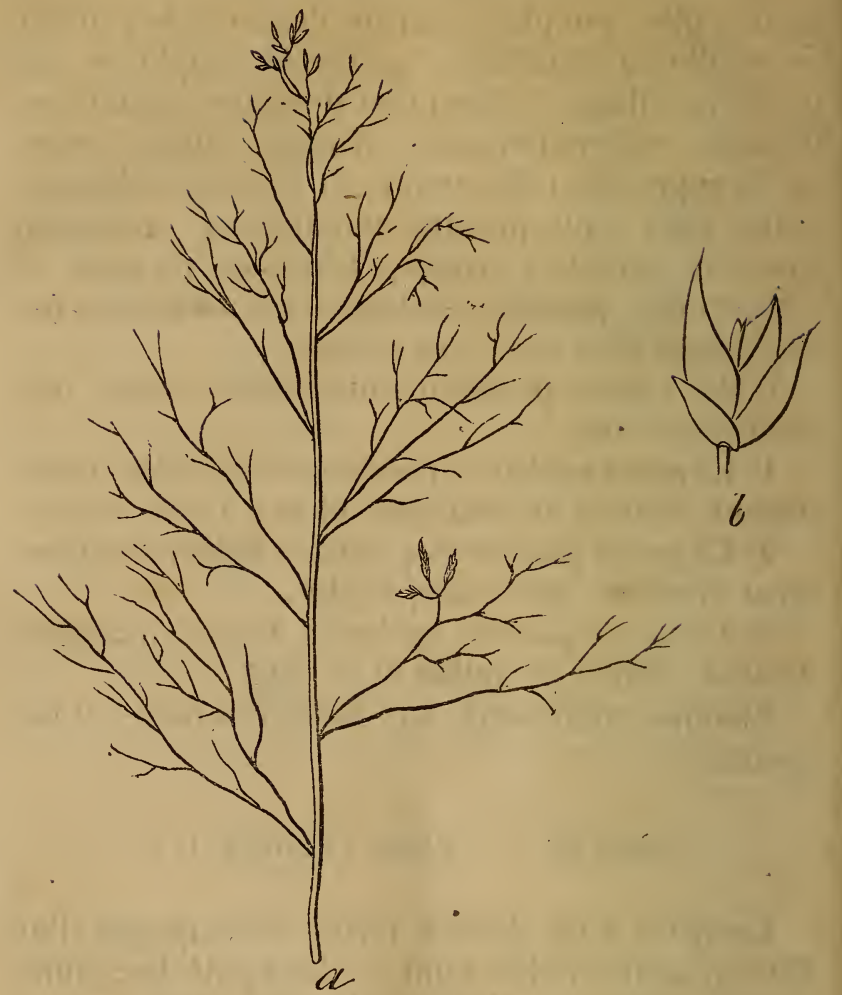

Fig. 58 (1).

AN.ILYSE DES ESPÈCES.

4) Souche rampante; vivace. . . . Panis b́levé (c).

1 Souche cespiteuse, annuelle . . . . 2.

( Chaume fort, d'un mètre environ de hauteur; panicule grande, lourde, 2 penchée au sommel : ..... Panis millet (a). Chaume de 5-5 décimètres; pani- Panis capllatane cule à rameaux capillaires, élalés . . (b).

(1) $a$ Panicule du panis miliacé ; $b$ locuste de panis miliacé ou millet des oiseaux, grossie. 
a)Panismillet.(Panicum miliaceum. L.) Souche annuelle, cespiteuse ; chaume fort, ascendant, de 7-9 décimètres ou plus de hauteur, velu; feuilles larges, hérissées de longs poils, principalement sur leurs gaines; panicule grande, penchée ; locustes glabres, orales, aiguës, d'un vert jaunâtre; grain luisant, blanc-jaunâtre, rougeâtre ou noirâtre, suivant les variétés. Annuel; fleurit en juillet. Cultivé.

b) Panis capillaire. (Panicum capillare L.) Souche annuelle, cespiteuse; chaume ne dépassant presque jamais $\mathbf{3}$-ว̃ décimètres de hautcur ; gaines des feuilles très-velues; panicule dressée à rameaux capillaires, formant un angle presque droit avec l'axe primaire de l'inflorescence; paillettes lisses ; paléole externe ovale-lancéolée, aiguë. Annuel; fleurit en juillet et en août.

Champs cultivés.

c) Panis élevée; Herbe de Guinée. (Panicum jumentorum. Pers; Panicum altissimum auct.) Souche vivace, rampante, émettant un assez grand nombre de chaumes de 2 à 3 mètres, droits, glabres; feuilles étroites, un peu rudes au toucher, longues d'un demi-mètre environ; panicule à 2-5 décimètres, très-rameuse, étalée, à rameaux verticillés, scabres; locustes verdâtres, subgéminées, ovales-oblongues; paillettes inégales, glabres; fleuron neutre à deux paléoles. Vivace; fleurit en août-septembre.

IVe sous-tribu. - Androgoponées.

Locustes géminées, l'une sessile hermaphrodite, l'autre pédonculée stérile; paléole externe du fleuron fertile aristée. 


\section{GENRE 55. - SORGHIER. (SORGHUM PERS.)}

Locustes géminées, dont l'une pédicellée uniflore mâle ou neutre, l'autre sessile hermaphrodite, biflore, l'un fleuron fertile et l'autre neutre ou stérile; glume à deux paillettes coriaces, l'inférieure à trois dents obscures; glumelle à deux paléoles ; l'inférieure subcoriace, bidentée au sommet, aristée entre les dents; arête tordue, pliée ; l'externe membraneuse; hyaline glumellule à deux paléolules émarginées; ovaire glabre; styles 2; stigmates 2, terminaux, plumeux; grain oblong, un peu comprimé ; panicule ample, très-rameuse.
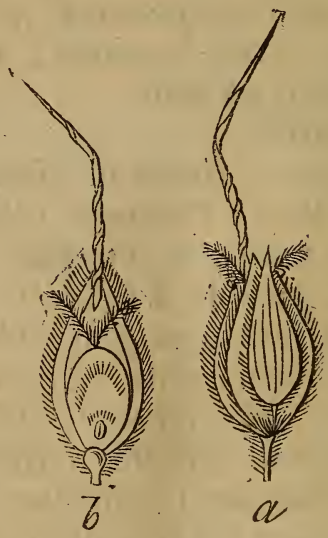

Fis. $59(1)$.

Sorghier vulgaire. (Sorghum vulgare Pers.; Holcus sorghum L.) Chaumes forts, articulés, pleins de moelle, de 1 à $\mathbf{3}$ mètres; feuilles larges, analogues à celles du maïs, pointues; nervure mé-

(1) a locustes géminées du sorghier vulgaire; $b$ locuste hermaphrodite du mème dont on a enlevé quelqques parties. 
DEUXIEME PARTIE. - ANALYSES ET DESCRIPTIONS. 253

diane forte, blanche; ligule courte, membraneuse; panicule grande, droite, un peu serrée; locustes d'un blane sale ou roussàtre; grain elliptique, arrondi, assez gros et de diverses nuances. Annuel; fleurit de juillet à septembre.

$$
\text { GENRE 56. - B.RBON. (INDROPOGON L.) }
$$

Locustes lancéolées, géminées, l'une sessile hermaphrodite, l'autre pédicellée mâle ou neutre par
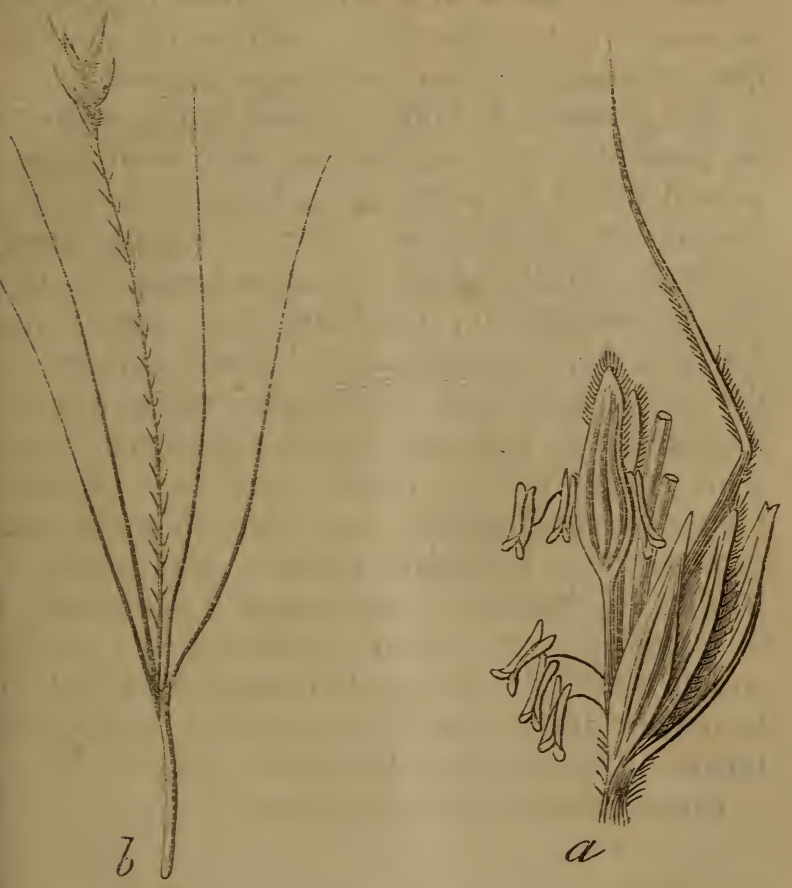

Fig. $60(1)$

(1) a Axe dïnfloreseence du harbon digité; $b$ lorustes gẻminces. 
avortement; locustes hermaphrodites : un fleuron fertile accompagné d'un second fleuron stérile, rudimentaire, se présentant sous la forme d'une paléole; glume à deux paillettes entourées de poils laineux; paillette extérieure arrondie ou à dos plan; paillette intérieure carénée ; paléole extérieure souvent réduite à une arête contournée, l'intérieure très-petite, quelquefois nulle; ovaire glabre; styles 2, terminaux; stigmates plumeux; grain oblong un peu comprimé.

Locustes mâles ou neutres : glume comme dans la locuste hermaphrodite ; paléole externe mutique, ordinairement solitaire, non carénée.

Axe primaire de l'inflorescence court, se divisant en plusieurs axes secondaires qui deviennent la base d'autant de petits épis, lesquels forment au sommet du chaume une panicule simple digitée.

Barbon digité. (Andropogon ischœmum L.) Souche subcespiteuse, émettant des racines nombreuses, dures et coriaces; chaumes rameux, redressés, à mérithalles inférieurs renflés, allant en s'amincissant jusqu'au nœud supérieur; nœuds d'un rouge violet; feuilles radicales étroites, planes ou canaliculées, parsemées de poils blanes et rares; les caulinaires glabres, plus larges à la base et se rétrécissant subilement à l'ouverture de la gaîne qui est poilue; ligule courte; panicule simple à 2-12 digitations d'un aspect blanchâtre ou brunâtre; arêtes d'un brun roussâtre, genouillées, longues. Vivace; fleurit de mai à août.

Lieux sablonneux et arénaires. 


\section{TROISIÈME PAR'IIE.}

\section{INICATIONS}

SUR

\section{LES STATIONS, LES PROPRIÉtÉS ET LE RENDENENT \\ DES GRANINÉES.}

Nous venons de passer en revue et d'étudier une à une toutes les espèces et les principales variétés degraminées céréales et fourragères que l'on rencontre en Belgique.

Il reste maintenant, dans cette troisième partie, à indiquer les qualités économiques et agricoles qui distinguent les espèces et les variétés les unes des autres, eu égard à leur rendement et à leurs propriétés nutritives.

Mais avant de passer à cet ordre de faits, nous croyons indispensable et urgent d'attirer l'attention des agronomes et des agriculteurs sur quelques précautions de la plus haute utilité, au défaut desquelles les meilleures acquisitions se perdent quelquefois au bout de peu d'années de culture.

Comme on le sait, un grand nombre de variétés de céréales cultivées ont entre elles beaucoup d'affinité. Eh bien, pour éviter toute confusion et tout doute sur la dégénération subite des céréales, il importe de se procurer la semence dans toute sa pureté et de recueillir avec tous les soins possibles celle dont on a besoin pour ses semailles. Pour atteindre sûrement le but qu'on se propose, la conservation des variétés, la science et l'art réclament, d'une part, leur connaissance parfaite basée sur des caractères certains, et, d'autre part, la récolte de la semence sur des plantes qui ont conservé toute leur 
intégrité, toute leur pureté spécifique : on y réussira en la recueillant sur les pieds les micux venus de panicule à panicule et d'épi à épi. En agissant de la sorte, et en changeant toutefois de sol et d'exposition, on ne conservera pas seulement la variétéavec toutes ses qualités, mais on l'améliorera d'une manière sensible au bout de quelques générations, et on aura la certitude de ne pas infester de mauvaises herbes le sol qui doit les produire.

Ces observations sont aussi applicables à la semence des graminées fourragères qui doivent servir à la formation de prairies sèches ou irriguées, car l'emploi du fond des fenils, où l'on rencontre presque toujours une infinité de graines inutiles ou nuisibles, est une pratique des plus vicieuses et à laquelle il faut renoncer. En effet, les prés, tout aussi bien que les guérets, ont une destination spéciale : les uns doivent fournir del'herbe réunissant un ensemble de bonnes qualités; les autres sont appelés à produire une récolte quelconque dégagéc de toute autre plante adventice. Or, s'il est contraire à une bonne exploitation d'avoir des guérets infestés de mauvaises herbes, pourrait-il être rationnel de voir les prés envahis par des plantes inutiles, médiocres ou nuisibles, qui pourraient être remplacées par des espèces plus nutritives ou plus productives? Cependant il n'arrive que trop souvent que les prés humides ou secs sont envahis par des espèces de qualité inférieure.

Il est donc de l'intérêt du cultivateur de veiller sur la productivité de son exploitation et de profiter de toutes les lecons dont la nature se montre si prodigue aux yeux de celui qui sait utiliser les recherches des botanistes; et l'agronome peut dans toutes les circonstances, avec les données exactes 
des stations que les végétaux recherchent de préférence, faire un choix convenable et productif parmi les espèces et variétés que nous allons examiner successivement.

Nous suivrons la marche que nous avons adoptée dans la partie qui précède, et nous indiquerons successivement ce que l'on a aequis sur les stations et les sols qu'elles affectionnent, le rendement (1) qu'elles fournissent, et ce que l'on sait comparativement de leurs propriétés nutritives (2).

(1) Les produits que nous indiquons ont été obtenus dans les meilleures conditions et doivent être considérés comme le maximum que les espèces dénommées puissent fournir, sauf quelques rares exceptions. Nous les empruntons aux travaux remarquables du comte de Gasparin. Le foin dont il est question contient encore 10 à $15 \%$ d'humidité et constitue le foin normal.

(2) L'étude des propriétés nutritives, corroborée par des expérienres directes, est encore très-peu avancée jusqu ici, en ce qui concerne les graminées fourragères. La théorie des chimistes et des physiologistes a conduit à des résultats dont les agronomes ont le plus grrand intérêt à vérifier le côté pratique, dans leurs étables, leurs pàturages et leurs pacages. Noas les empruntons à II. de Gasparin. Cettc théorie, comme on le sait, a pour point de tépart les matičres azotées et considère les plantes comme d'autant plus nutritives qu'elles contiennent plus d'azote. Toutefois, nous ferons observer que la même plante obtenue dans des stations diverses fournit des résultats différents; ce qui d'ailleur's s'explique facilement. On se gardera aussi de confondre les propriétés nutritives avec les propriétés engraissantēs, qui sont deux choses très-distinctes.

Pour faciliter la comparaison des qualités nutritives, nous les réunissons ci-après dans un seul tabicau.

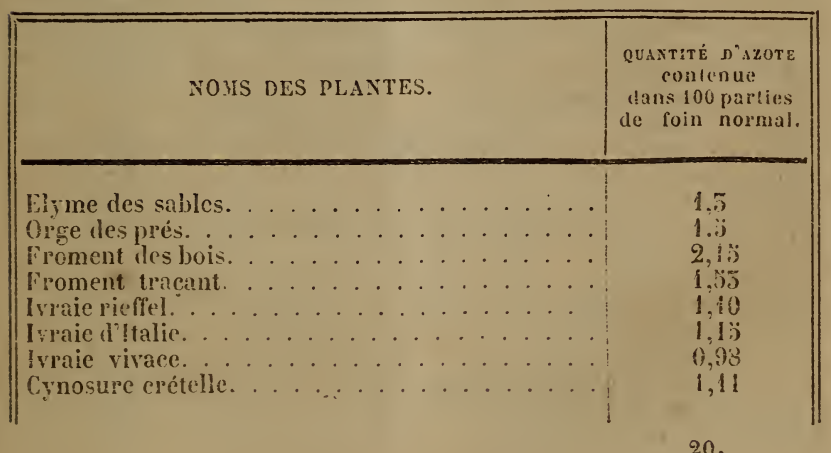

20. 
Après avoir épuisé ce cadre, et comme conclusion, nous grouperons les meilleures espèces qu'il convient de choisir pour les diverses stations qui peuvent se présenter dans la pratique.

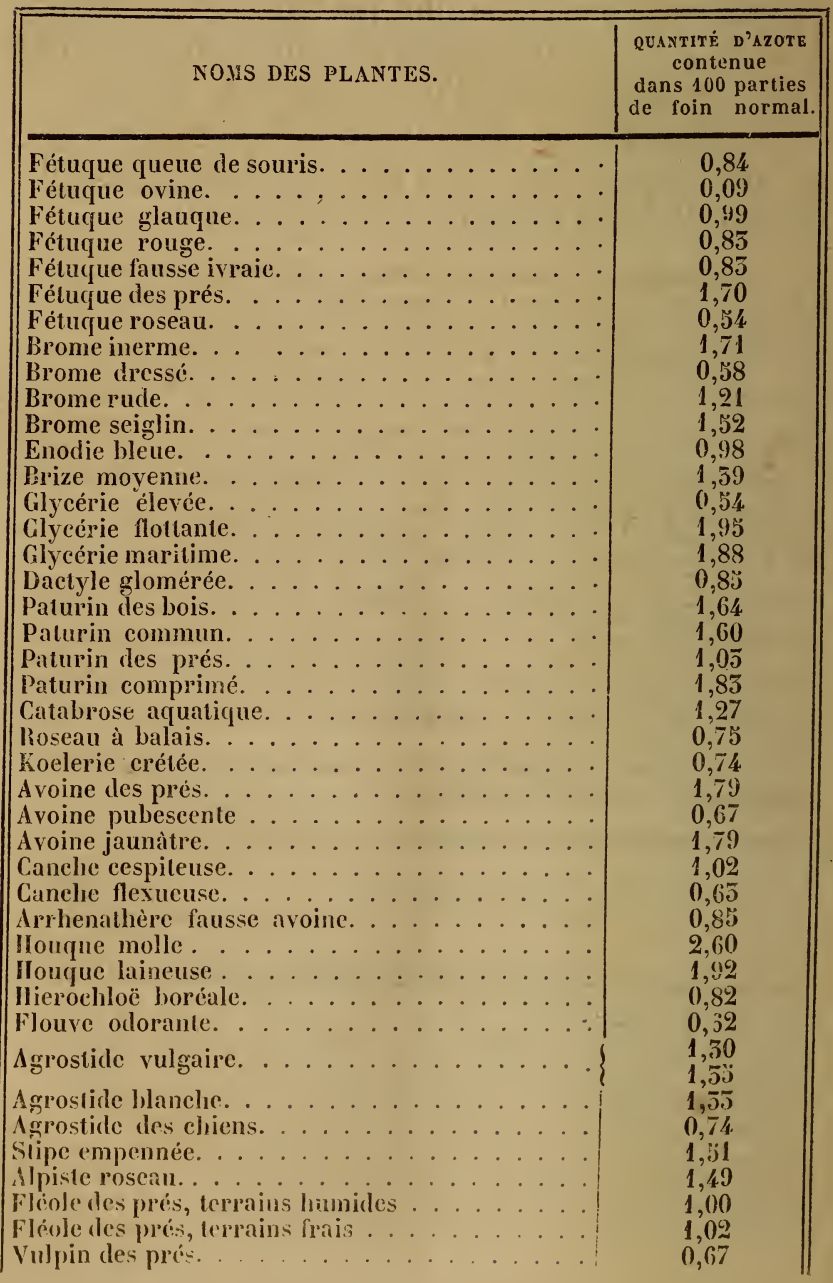


gre tribn. - Raydés.

Mays cultivé. Le maïs cultivé est une plante annuelle qui aime un sol fertile, plutôt glaiseux que sablonneux, et qui demande une bonne exposilion chaude.

Quelques variétés peuvent être cultivées pour leur grain; ce sont : le maïs quarantain, le maïs à poulet et le maïs à bec. De ces trois variétés, le maïs à poulet est le plus précoce, mais le moins productif, à cause de la petitesse de ses grains ; ensuite le maïs à bec, qui est aussi hàtif que le maïs quarantain, mais plus productif que celui-ci.

Les autres varićtés ne peurent être cultivées en Belgique que comme espèces fourragères qui donnent un produit très-considérable et de très-bonne quålité tant en rert que sec. On les coupe lorsque les panicules de fleurs màles commencent à monttrer leurs pointes, jusqu'à la pleine floraison.

La farine de maïs, qui forme la base d'une bonne nourrilure pour les animaux à l'engrais, depuis la poule jusqu'au bouf, n'est pas propre à la panification ; le pain qui en est fait se durcit considérablement en très-peu detemps : il est d'ailleurs lourd etassez indigeste, àmoins que sa farine ne soit mêlée arec celle de froment. La farine de maïs contient environ 70 p. c. d'amidon, 12 p. c. de gluten et 10 p. c. d'huile grasse. L'hectolitre de grain de maïs pèse de 66 à 69 kilogrammes.

Ig tribn. - Triticacées.

Elyme des s.tbles. - Virace, traçante.

Stalions. - Bords de la mer, dunes, ou il fixe par ses rhizomes tracants les sables maritimes, el terrains sees. 
Propriétés. - Les ruminants (boufs, moutons, chèvres, etc.)le broutent quand il est jeune; quoique riche en matière sucrée, ils ne touchent plus ni à ses chaumes, ni à ses feuilles, à cause de leur dureté, lorsqu'ils ont acquis leur entier développement.

Rendement. - Il peut donner 27,565 kilogrammes de foin.

Elyme géniculé. - Mêmes observations.

Elyme d'Europe. - Vivace.

Stations. - Terrains secs et humides, calcaires et schisteux; bords des routes, bois et lieux ombragés des montagnes.

Propriétés. - Les ruminants l'aiment beancoup quand il est jeune.

Rendement. - Les trois espèces qui précèdent perdent environ la moitié de leur poids par la dessiccatation; le foin constitue un fourrage des plus médiocres.

Orge des prés. - Vivace.

Stations. - Terrains loameux sujets aux débordements, prés humides, pâturages gras et prairies.

Propriétés. - Plait à tous les animaux dans sa jeunesse, mais fournit un fourrage qui n'est pas sans danger lorsque les épis sont formés (nous entendons par là que les arêtes ont acquis de la consistance), à cause des arêtes munies de denticules qui s'introduisent dans le palais et sous la langue et les font beaucoup souffrir.

Rendement.-Peu productive, perd environ les trois cinquièmes par le fanage.

Dans des cas exceptionnels, le rendement s'élève à 3,67 hilogrammes.

Orge des souris. - Annuelle.

Stations. - Terrains loameux, décombres. 
TROISIENE PARTIE. - STATIONS, ETC. 241

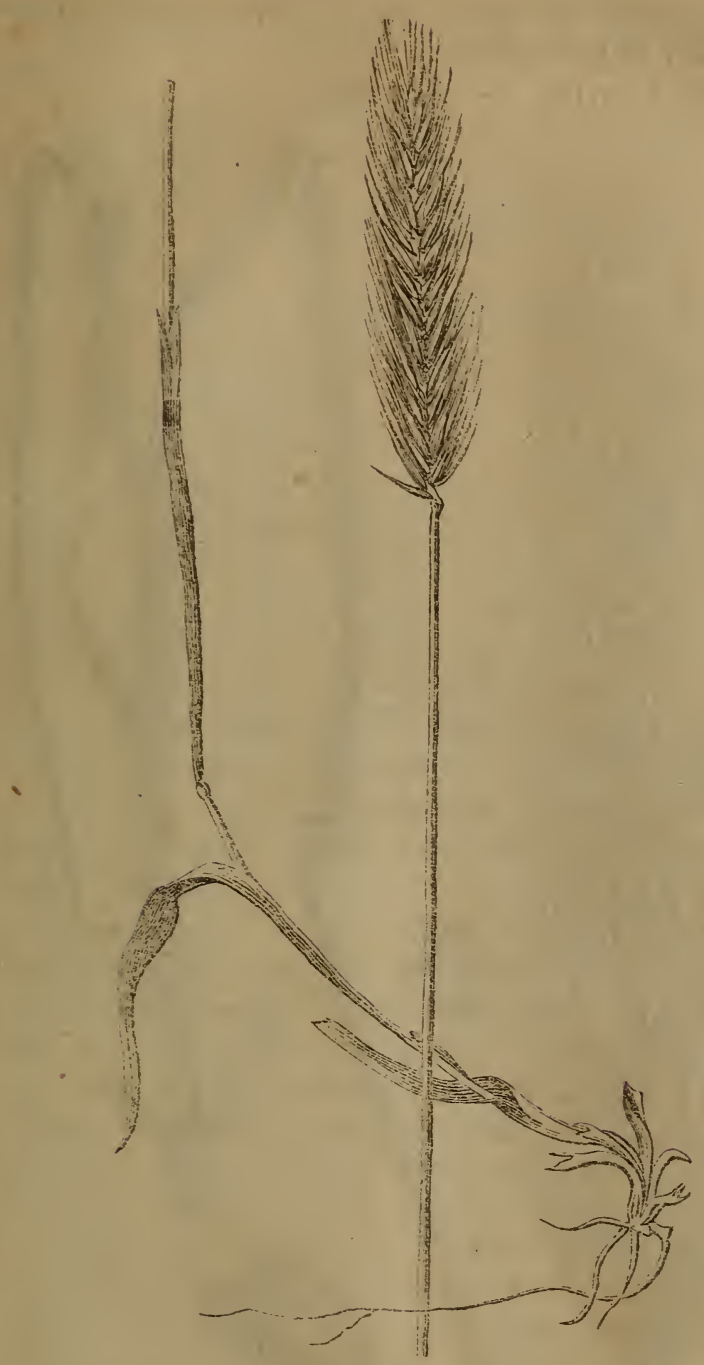

Yis. (t) 
Propriétés et rendement. - Mèmes observations que pour la précédente.

Orge maritime. - Annuelie.

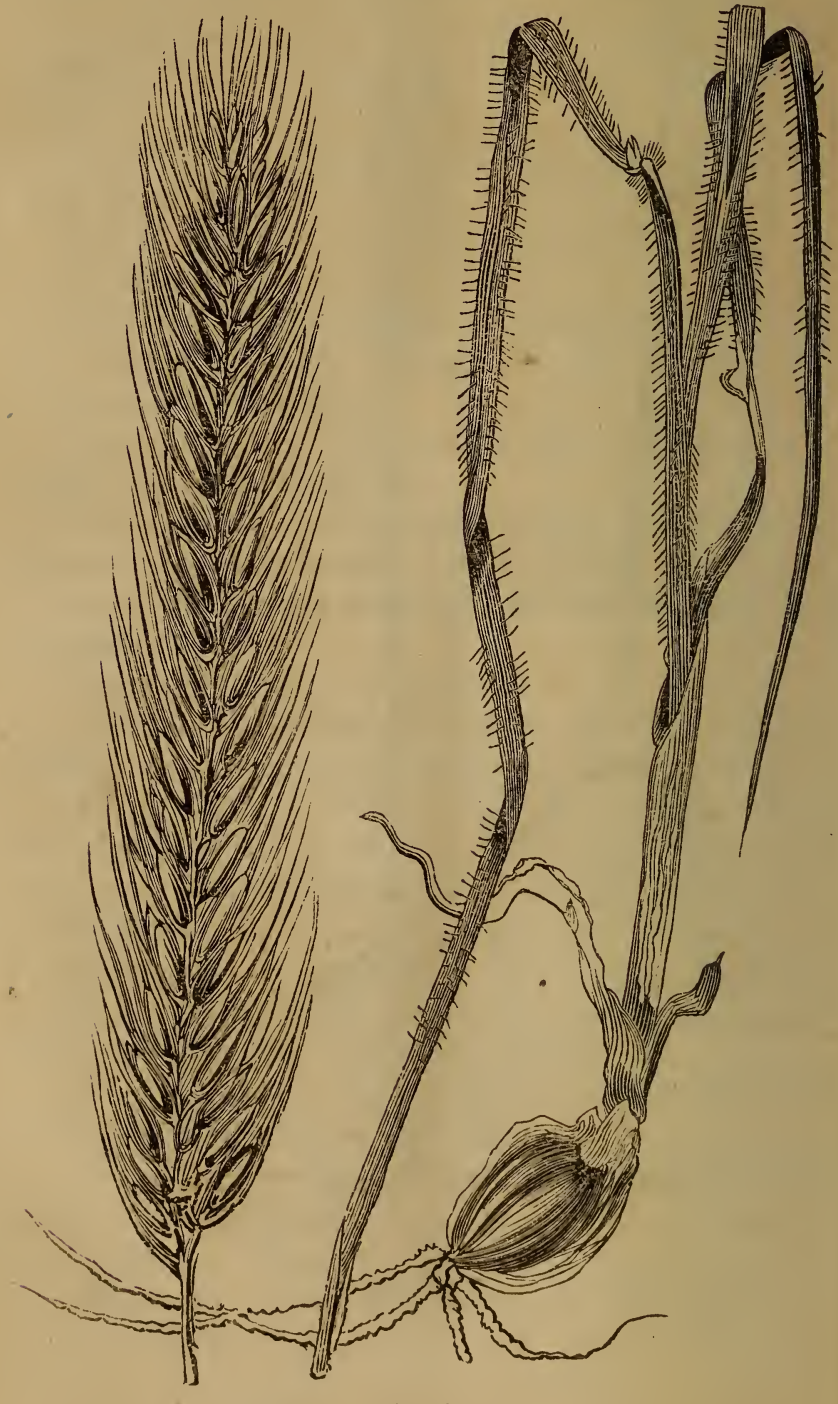

Fig. 62.

Orge bulbeuse. 
Stations. - Dunes, sables maritimes.

Propriétés. - Plait aux bestiaux quand elle est jeune.

Rendement. - Très-peu productive, perd les deux tiers de son poids par la dessiccation.

Orge bulbeuse. - Vivace. (Fig. 62.)

Stations. - Terrains loameux fertiles, profonds.

Propriétés. - Très-bon fourrage tant vert que sec : doit ètre coupée avant la formation de l'épi; très-bonne espèce pour la formation de prairies artificielles de longue durée.

Rendement. - Cette orge donne un grand produit et est extrèmement précoce; elle peut fournir dès la mi-mai une première coupe abondante et deux coupes ultérieures non moins productives: elle perd environ les deux tiers de son poids par la dessiccation, qui s'opère difficilement. Le rendement monte souvent à 20,000 kilogrammes.

Orge distique. - Annuelle; d'élé.

Stations. - L'orge distique, comme toutes les orges en général, aime une terre loameuse où le sable prédomine.

Propriétés. - De toutes les orges distiques cultivées, celle qui fournit le plus grand produit et de meilleure qualité est sans contredit la variété dite orge chevalier parmi les vêtues; l'orge nue, appartenant à cette espèce, dont on peut recommander la culıure comme la plus productive et la meilleure, est la variété dite grosse orge nue.

Orge pìramidale. - Annuelle; d'été.

Stations. - Terraius plats, loameux; mais réussit cependant aussi dans les terrains compactes, froids, et résiste également bien à la sécheresse. 
Propriétés. - Son grain est bien nourri et lourd.

Rendement. - Produclive.

Orae a six rangs. - Annuelle; d'hiver.

Stations. - Liorge à six rangs prospère dans un terrain loameux fertile.

Propriétés. - Elle se cultive comme plante granifère el fourragère. Elle est plus productive en grains que toutes les autres orges, mais sa qualité parait être un peu inférieure à l'orge vulgaire. Comme plante fourragère, celle-ci est infiniment préférable.

Orge vulgare. - Escourgeon.

Stations. - Elle exige un terrain meuble profond et fertile.

Propriétés.-Celleorge se cultive comme plante granifère et fourragère.

Parmi les variétés vêtues les plus productives et les plus recherchées, on peut citer :

L'orge commune d'hiver et l'orge carrée du printemps.

L'orge céleste, parmi les nues, est celle qui doit mériter la préférence sous le rapport de la qualité et du produil; elle cst de mars. Comme fourragère, l'orge vulgaire sera toujours préférée à cause de l'abondance du produit. C'est une herbe des plus succulentes, recherchée par tous les bestiaux; elle exerce une influenee salutaire sur la sécrétion laiteuse qui gagne considérablement en quantité et en qualité; tous les jeunes animaux s'en trouvent très-bien, de mème que ceux qui sont échaufés par les fatigues.

Ces propriétés sont connues depuis des sic̀cles; elles nous ont été transmises par lillustre Olivier de Serres qui s'exprime en ces termes : 
"A vec le seul orge chevalin ou d'hiver, faict-on aussi de bon farrage. On sème cet orge, quand et en semblable temps quel'autre farrage, et de mème le bestail le paist en campagne durant l'hiver. Si de ce l'on se veut abstenir, gardéjusquesau printemps; cet orge est fauché ou moissonné en herbe, mais petit à petit, pour de jour à autre le faire manger aux chevaux, dont profitablement ils se purgent, de là prenant le commencement de leur graisse. Tout autre bétail, gros et menu, s'en porte aussi très-bien, si on le paist modérément de cette herbe, car de leur en donner à discrétion scrait en danger de s'en trouver mal, par trop de replection, tant abondante est-elle en substance. Couppé à la fois, cestorge, en herbe, seché etserré au grenier, comme l'autre foin, est aussi bonne viande (nourriture), pour tout bestail en hiver, et arenant que la couppe en soit faicte comme sur la fin d'avril ou commencement de maix, le reject de ses racines, conservé, produira gaillardement nouvelle herbe et de grain arec, le temps n'estant extraordinairement chaud."

La paille peut aussi servir comme fourrage trèsmédiocre, à moins qu'elle n'ait été récoltée sur un terrain élevé et chaud; dans tous les cas, la paille d'avoine lui est préférable : un hectare donne de 1,600 à 1,900 kilogrammes. L'hectolitre d'orge d'été pèse de 59 à 66 kilogrammes; l'orge d'hirer pèse de 63 à 70 kilogrammes. La farine contient environ 60 p. c. de fécule et un vingt-quatrième de gluten et d'albumine.

Tout le monde connaît les usages variés de toutes les orges cultivées : l'orge esı une céréale indispen. sable dans la fabrication de la bière; elle forme la hase de la boisson désignée sous le nom d'orgeai. Le peu de gluten qu'elle renferme fait que le pain 
en est indigeste et lourd, quoiqu'il paraisse plus nourrissant que le pain de seigle; il ne peut convenir qu'aux personnes qui se livrent à des travaux fatigants. Dans l'antiquité, les gladiateurs en faisaient en quelque sorte leur nourriture exclusive; aussi une mesure d'orge était-elle la récompense des athlètes vainqueurs à ces jeux de la Grèce.

On la réduit en gruau pour en faire des polages d'un goût assez agréable, des bouillies et des lisanes rafraìchissantes.

L'orge mondé est le grain dont on a enlevé les paléoles et le péricarpe (pellicule) et arrondi les extrémités.

L'orge perlé est celle qu'on a réduite en grains demi-transparents, polis comme une perle; il est moins âcre et moins amer que l'orge mondé et plus propre à être employé, en guise de riz, avec le lait et le bouillon.

L'orge est aussi une bonne nourriture pour les chevaux et les bestiaux à l'engrais.

Une orge, pour être bonne, doit être pesante, à surface unie, non ridée, à pellicule mince. Les orges d'hiver sont préférées aux orges d'été par les brasseurs : pour les distinguer entre elles, ils ont recours à l'examen de la scobine, qui est fortement ciliée ou poilue dans les orges d'hiver, tandis qu'elle est presque glabre dans les orges d'été. Il y a des variétés qui peuvent facilement induire les négociants en erreur. Il est donc plus sùr de recourir à la balance, qui ne trompe jamais sur le poids réel ou la densité d'une marchandise.

Seigle cultivé. - Annuel.

Stations. - Le seigle aime un sol profond, meuble, sablonneux ou loameux; il ne réussit pas si bien dlans les terres fortes, froides et compactes. 
Le seigle de Rome est de toutes les variétés celle qui présente le plus de qualités comme plante granilère. (Fig. 63.)

Le scigle de Russie a été recommandé vivement, dans ces derniers temps, comme donnant deux ou trois coupes de fourrage la première année, et, par conséquent, comme étant plus productif qu'aucune autre graminée fourragère. Sans entrer dans des déreloppements sur celte théorie, il nous semble, ainsi qu'Oscar Lecler'q l'a très-judicieusement fait remarquer, que le semis en juin, qui a été préconisé par M. Lecocf, ne pourrait avoir lieu sans mettre à sa charge la rente entière de l'année, puisque aucune récolte de plantes sarclées n'a encore été faite à cette époque, ce qui ne serait nullement avantageux. Aussi, d'après quelques expériences que nous avons faites, nous rangeons-nous de l'avis de M. de Gasparin : " que le maïs fourrage lui est bien supérieur ; mais, continue-t-il, ce seigle, comme le seigle d'hiver, et mieux que lui sans doute, peut offrir des ressources au printemps par la précocité de sa pousse; il donne, mieux que toute autre plante, un fourrage abondant et précieux dans cette saison. "

Les dernières expériences qui ont été faites en Angleterre et répétées récemment dans notre pays, ont démontré que le seigle est un peu moins nutritif que l'orge et moins bon fourrage qu'elle pour les vaches laitières, les chèvies et les moutons. C'est ce que tous les herbagers savent depuis longtemps.

Cependant, nous n'hésitons pas à recommander comme fourrage de printemps la culture du seigle de Russie, semé à la mème époque que les autres - seigles.

Le seigle fournit un pain lourd et compacte qui 
convient aux personnes habituellement constipées; il est einployé comme toutes les autres céréales dans les distilieries; sa farine en cataplasme est résolutive.

- La paille de seigle sert de base à la litière de nos bestiaux, à faire des liens et des paillasses, à empailler les chaises et à couvrir les habitations rustiques. Elle peut aussi servir à l'alimentation des moutons et du gros bétail, quand on la mêle avec du trèfle, de la luzerne, etc.

Un hectolitre de seigle pèse de 69 à 76 kilogrammes; la farine contient environ $64 \mathrm{p}$. c. damidon, 10 p. c. de gluten et d'albumine, 11 p.e. de gomme, $51 / 2$ p. c. de matières grasses et 3 p. c. de sucre.

Fromext cultivé. - Annuel.

Stations. - Toutes les varićtés de froment cullivé aiment une terre argileuse, forte, pour donner un grain bien nourri, riche en fécule et en gluten.

Propriétés. - Parmi les variétés de froment d'hiver roux, nous recommandons : $1^{\circ}$ le froment de Brabant roux; 2 le froment à balle rouge de Dantzich; $5^{\circ}$ le froment rouge d'or (Mary gold red); $4^{\circ}$ le froment rouge d'Ecosse.

Ces deux dernières ne sont que des sous-variétés du froment de Brabant modifié en Ecosse, et demandent une terre fertile, argilo-glaiseuse.

Parmi les froments d'hiver blancs: $1^{\circ}$ le blanzé de Flandre, avec sa sous-variété dite blé wittington, qui ne convient que dans les honnes terres fraiches; $2^{\circ}$ le froment hichling etses sous-variétés, et $5^{\circ}$ le froment velouté blanc, dit blé tunstall.

Les froments de mars mulques, qui méritent d'ètre recommandés à l'allention des agriculteurs, 
TROISIÈ PARTIE. - STATIONS, ETC.

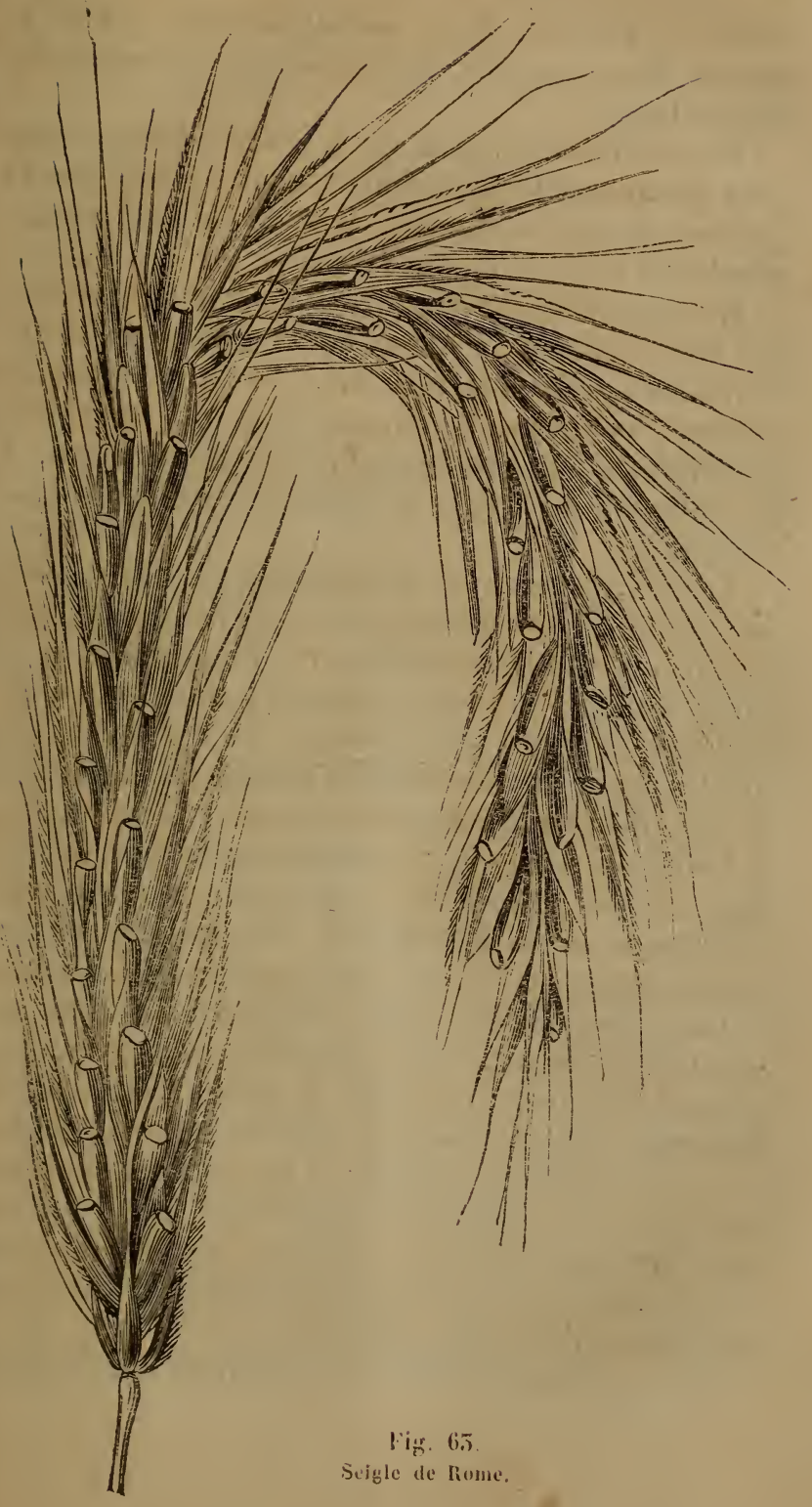


sont : $1^{\circ}$ le blé de Talavera de Bellevue, et $2^{\circ}$ le blé de Marianopolis; mais on doit les semer de bonne heure.

Les variétés aristées sur lesquelles l'expérience s'est prononcée favorablement, sont : $1^{\circ}$ le froment de mars barbu ordinaire, et $2^{\circ}$ le blé du Caucase amélioré, et $3^{\circ}$ le blé hérisson.

Les variétés appartenant au froment cultivé sont les plus estimées, à cause de la finesse et de la blancheur de leur farine; mais le sol, le mode de culture, les influences climatériques et l'exposition coniribuent pour beaucoup à l'augmentation ou à la réduction du chiffre des éléments contenus dans le grain.

Les chimistes et les physiologistes, pour estimer la qualité d'une céréale quelconque, s'adressent au chiffre des matières azotées et l'évaluent selon sa plus ou moins grande richesse en gluten et en albumine. D'après ces domnées, certaines espèces, que les praticiens considèrent comme de qualité médiocre, devraient occuper le premier rang. Ainsi, le blé de miracle, qui est en quelque sorte repoussé des marchés, contient, à en croire les analyses chimiques de M. Rossigneux, corroborées par les raisonnements de $\mathbf{M}$. le comte de Gasparin, autant, si pas plus, de gluten que les espèces les plus renommées de notre pays; cependant, tous les jours, on voit diminuer la culture de cette variété qui est beaucoup plus productive dans les sols riches que le meilleur froment cultivé. Une observation dont nous pouvons garantir l'exactitude, e'est que la pâte, dans la fabrication du pain, s'élend, au lieu de se gonfler, en fermentant el fournit un pain peu aggréable et d'un goût douceâtre.

Quoiqu'il en soit, les froments roux contiennent 
plus de gluten que les froments blancs et donnent un pain plus lié et plus nutritif.

Les qualités que l'on doit rechercher dans le froment sont un grain bien nourri, bien développé et un sillon très-étroit à bords droits.

L'hectolitre de froment pèse de 76 à 80 kilogrammes. Dansquelques cas très-rares, on a vu ce chiffre atteindre environ 100 kilogrammes. La farine contient environ de 79 à 86 p. c. de fécule et de cellulose et 12 à 26 p. c. de gluten et d'albumine.

Le froment fournit encore à l'économie agricole sa paille qui est considérée à juste titre comme la plus nourrissante ; elle est presque toujours améliorée par la présence de diverses plantes légumineuses, telles que les vesces, les gesses, le trèfle, etc., qui croissent naturellement dans les guérets; mais elle est aussi quelquefois dépréciée par la présence de divers chardons, centaurées (le blenet, etc.), agrostemme, calystégie, etc. La paille qui est couverte de lignes ou de taches rousses, jaunes-rouges, noirâtres ou noires, doit être rebutée; car elle pourrait faire naitre des accidents sérieux chez les animaux qui s'en nourriraient.

Pour qu'elle soit de première qualité, la paille doit avoir une couleur jaune, vive ou dorée, et ne présenter aucune odeur de moisissure ou de champignon ; màchée, elle doit avoir une saveur légèrement sucréc. Les pailles des céréales d'hiver sont plus recherchées par les bestiaux que celles des variétés du printemps, et celles récoltées sur un terrain élevé et chaud sont meilleures que celles des terrains bas et froids. La paille est utilisée en nature ou hachée; ce dernier procédé , lorsqu'clle 
estmêlée à d'autres aliments, est le plus économique et le plus profitable.

275 kilogrammes de paille équivalent à $100 \mathrm{ki}-$ logrammés de bon foin.

Fronent turgide. - Annuel.

Stations. - Le froment turgide et ses variétés exigent un terrain loameux, humide, fertile, où les autres blés verseraient ou se rouilleraient.

Propriétés. - De toutes les variétés à épi simple, celles connues sous les noms de pétanielle noire de Nice et de poulard blanc, ou roussâtre velouté, sont de beaucoup les plus productives; mais la qualité de leur farine est médiocre. Celle du poulard blanc est bise; l'autre est un peu grisâtre, rude et grossière, analogue à celle du blé de miracle, dont le rapport est considérable, mais qui est difficile sur le terrain et assez délicat à l'hivernage. Aucune des trois n'est propre à faire un bon pain blanc, lapâte n'ayant pas de corps et étantgrisâtre; mais, vu l'abondance de leur rendement, elles peuvent servir utilement à la confection du pain de méteil et à l'alimentation des animaux à l'engrais : leur grain a un. dixième de valeur moindre que celui du froment cultivé. La nonette de Lausanne, qui a été rebaptisée du nom de géant de Sainte-Hélène, est très-productive et présente un grain glacé très-propre à faire des semoules.

Toutes ces variétés produisent une grande masse de paille sur la nature de laquelle les éleveurs ne s'accordent pas. Il en est qui la considèrent comme très-nutritive ; d'autres, au contraire, la trourent de qualité médiocre. Malgré cette dissidence d'opinion, les bestiaux la mangent avec plaisir.

Fronent dur. - Annuel.

Stations. - Le froment dur ne réussit bien que 
dans les pays chauds et sur des terrains élevès et fertiles.

Propriétés. - La trimenia de Sicile, qui est un blé de mars, se montre peu difficile sur le terrain; le blé de Xerès est plus exigeant.

On s'est demandé s'il n'y aurait pas d'avantage à préférer l'une de ces variétés au froment culiivé a grain tendre. Toutes les especes dont nous arons tenté la culture dans les stations les plus varices, ne nous ont domé aucun résultat qui puisse en recommander la culture. D'abord, elles mùrissent difficilement sous le climat belge, le grain n'acquiert point son complet développement, le sillon est ouvert, et la dureté va toujours en diminuant; ensuite, quoique la farine en soit riche en gluten et en amidon, sa valeur vénale est au moins un dixième en dessous de celie des blés lendies.

Froment de Pologne. - Toulce que nous venons de dire des blés durs s'applique au froment de Pologne, sauf que son grain est encore plus blane et un peu plus riche en gluten et en amidon. Son rendement est insignifiant.

Frouent épelutre. - Annuel.

Stations. - Cette espèce aime les sols profonds, un peu humides et fertiles ; cependant, il ne redoute pas la sécheresse.

Propriétés. - L'épeautre rouge doit ètre préféré aux autres, parce qu'il résiste très-bien à l'humidité et au froid, qưil talle mienx, quiil pousse des chaumes plus forts et plus devés, et qu'il donne des épis bien développés et une farine plus belle et plus liante.

Rendement. - L'épeautre rend de 52 à 60 heetolitres de grain vêlu à Thectare. 


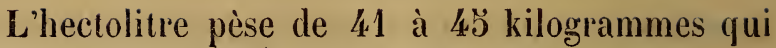
donnent de 30 à 34 kilogrammes de grain net.

L'épeautre présente un grain plus nutritif et d'aussi bonne qualité que le meilleur froment cullivé. On en jugera par l'analyse suivante, faite par M. Boussingault :

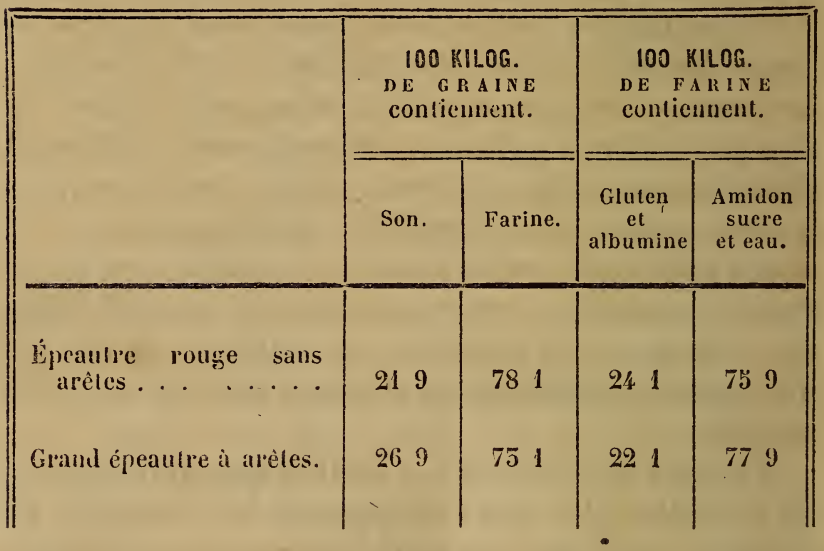

Froment amidonnier. - Annuel.

Stations. - Il aime une terre loameuse, meuble et fertile.

Propriétés. -- Cette espèce est cultivée en vue de la grande quantité d'amidon qu'elle peut fournir; elle se distingue par la beauté de son ggrain et la bonne qualité de la farine très-riche en fécule.

Frouent engrain. - Petil épeautre. - Annuel.

Stations. - Cette plante peu productive aime un sol fertile, sablonneux, situé sur une hauteur; mais il réussit aussi dans d'autres conditions.

Propriétés. - Son grain qui est subglacé fournit le meilleur et le plus fort de tous les gruaux. 
Rendement. - Comme ce petit épeautre réussit sur de mauvais terrains, soit sablonneux, siliceux ou crayeux, il constitue une bonne ressource pour les localités qui abondent en ce genre de sols où d'autres plantes ne prospéreraient pas. II fournit aussi une grande quantité de fourrage dont les bestiaux sont friands.

Froment des bois el froment pinné. - Vivaces.

Stations. - Se trouvent dans les sols sablonneux, calcaires; dans les bois, sur les collines sèches, dans les plaines arides et les buiśsons.

Propriétés. - Les animaux ne les broutent qu'avant le déreloppement des chaumes qui se montrent vers le mois de juillet; les moutons s'en accommodent dans leur jeunesse.

Rendement. - Peu productifs, ils perdent environ la moitié de leur poids par la dessiccation. Quelques agronomes élèvent le rendement de ces espèces de 18,000 à 22,000 kilogrammes de foin; nous croyons que ces chiffres sont exagérés.

Fronent cilié et froment nard. - Annuels.

Stations. - Ils aiment un sol sablonneux ou calcaire et n'ont aucune utilité comme fourrage.

Le Fronext roide et le Fronent des CHIENS aiment les lieux ombragés des bois, où ils poussent avec beaucoup de vigueur un grand nombre de fascicules de feuilles stériles qui forment un bon fourrage. 


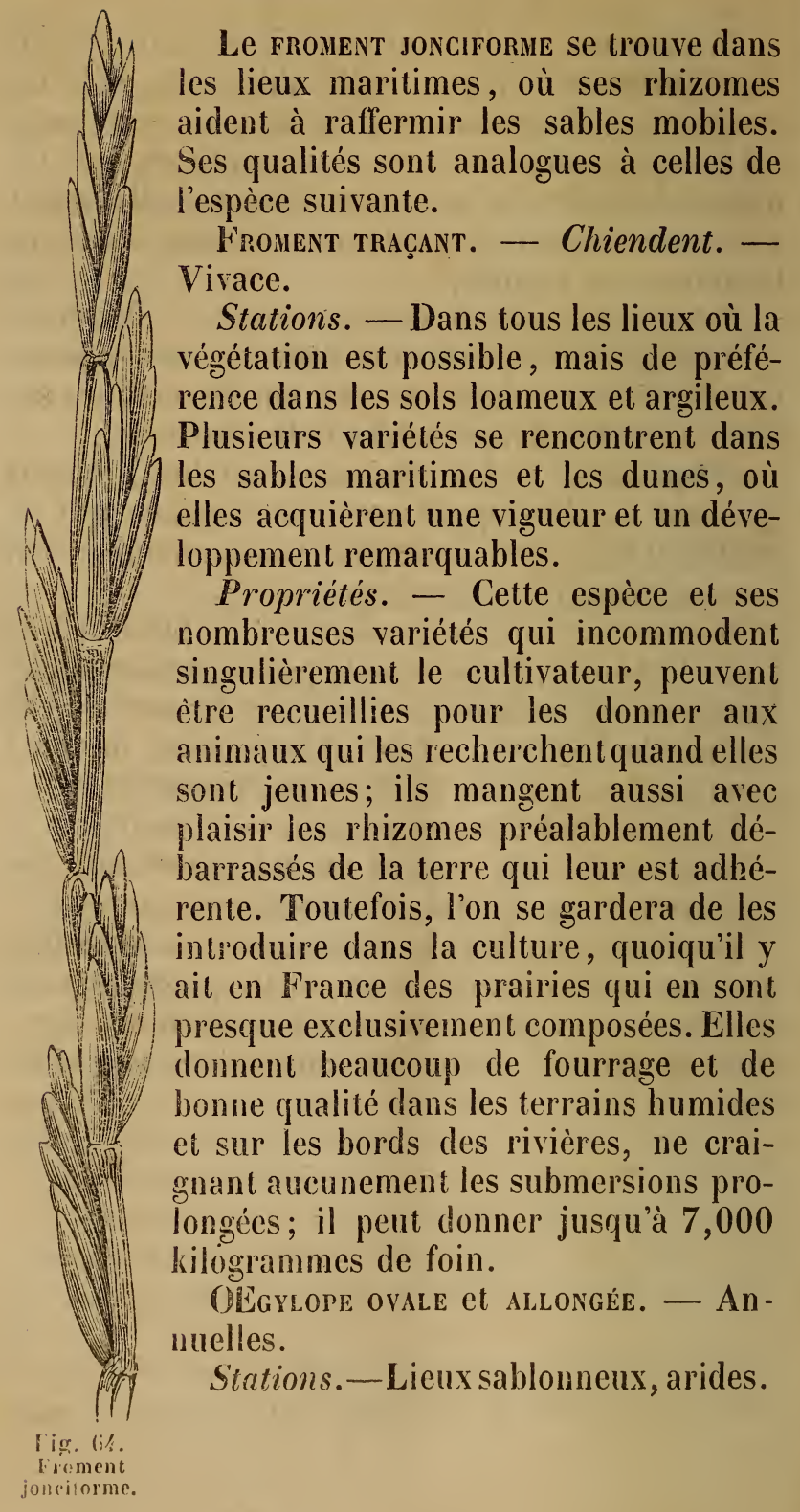




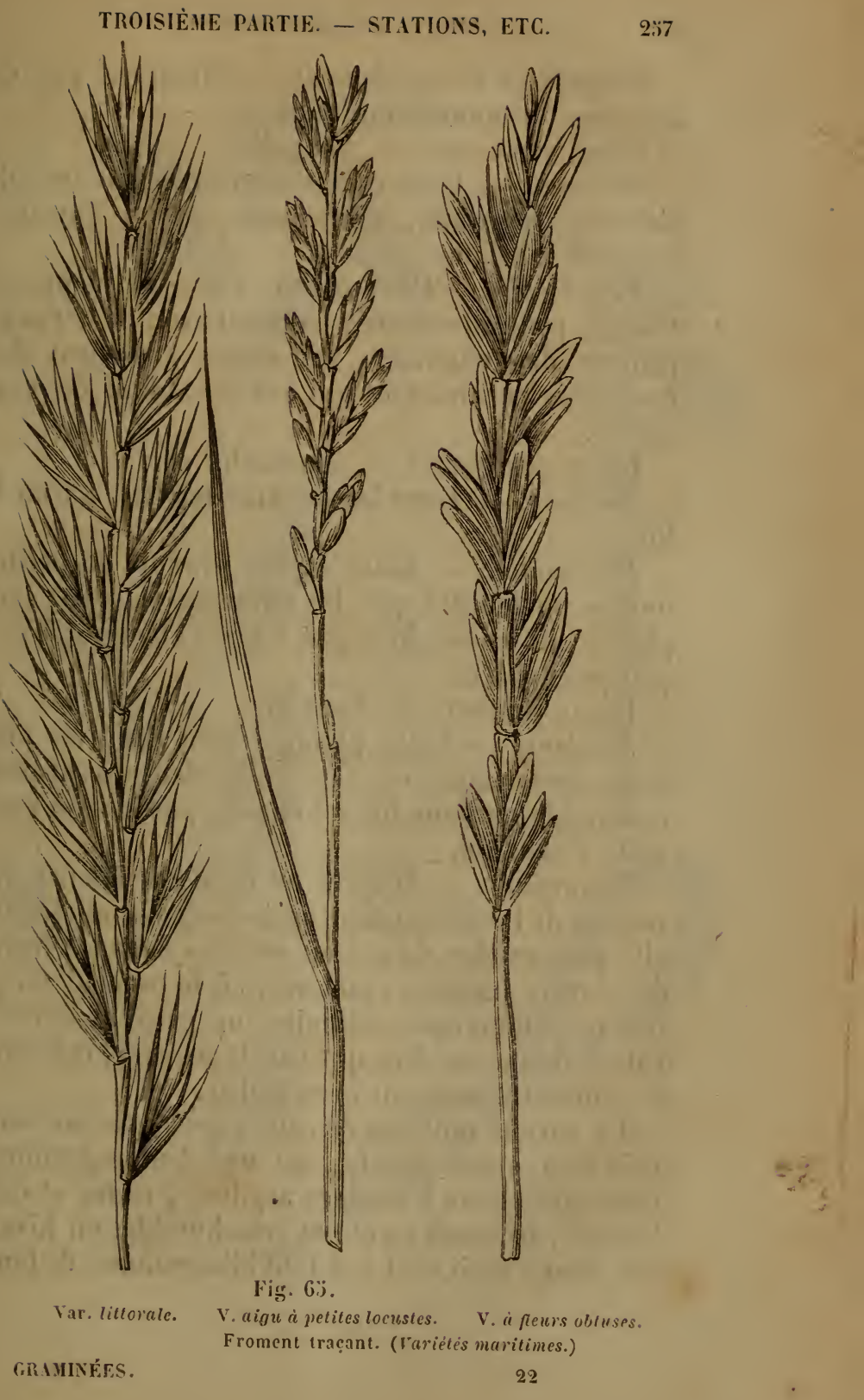


Propriétés et rendement. - Donnent peu de fourrage, de qualité médiocre.

Inraie enivrinte. - Anmuelle.

Stations. - Celte espèce se troure dans les sols glaiseux, schisteux, sablonneux, parmi l'avoine, l'orge et le seigle.

Propriétés. - Cette plante, qui se déreloppe en touffes, peut ètre donnée au bélail avant le développement des chaumes; ses grains possèdent des propriétés pernicieuses qui ont cependant été exagérées.

Ivraie des champs. - Annuelle.

Stations. - Dans les terrains fertiles, parmi le lin.

Propriétés. - Cette espèce produit de petites toufles de feuilles que les animaux mangent arec plaisir; ils ne touchent pas a leurs chaumes. Trèspeu productive.

IVraie Rieffel. - Annuelle.

Stations. - Cette plante régète dans les plus mauvaises conditions, c'est-à-dire dans un sol froid et humide ou dans un sol chaud, paurre, où l'eau reste stagnante.

Propriétés. - D'après les essais de Rieffel, directeur de l'établissement agricole de Grand-Jouan, elle peut rendre de grands services dans les terres de bruyère humides, maigres, où le trèfle ni aucun des bons fourrages ordinaires ne peurent réussir. Elle y donne un foin qui parait grossier, mais que les animaux mangent avec plaisir.

La variété mutique de cette espèce, encore nommée Ray-grass Bailly, est une bonne graminée fourragère dans les sables argileux, rudes et caillouteux, très-secs en été et très-humides en hiver; elle donne de $\$, 000$ à 8,000 kilogrammes de four- 
TROISIEUE PARTIE. - STATIONS, ETC.

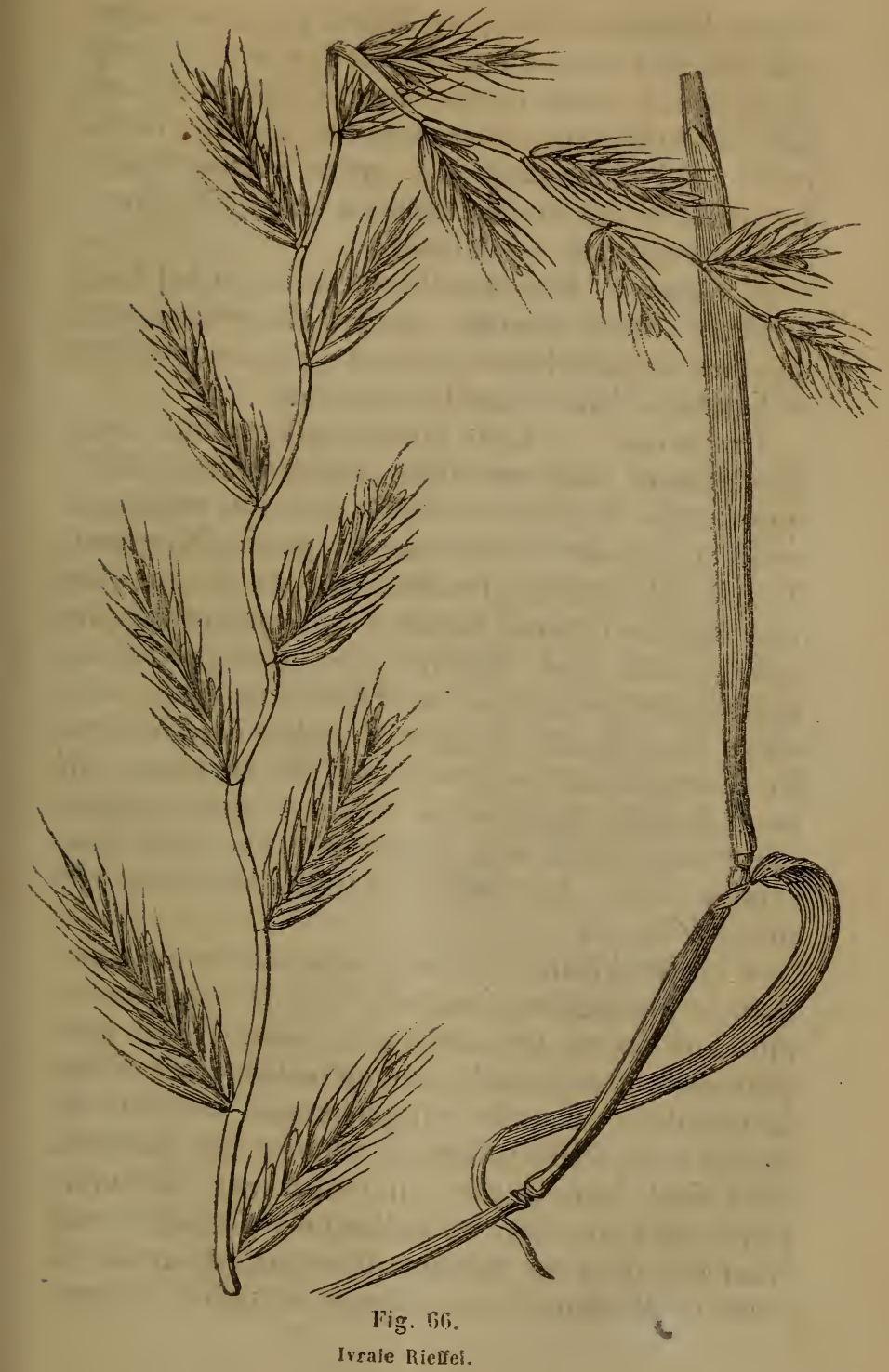


rage à l'hectare. Elle peut fournir, comme la précédente, une coupe abondante et une seconde coupe insignifiante. Pour emblaver un hectare, on emploie 30 kilogrammes de semence d'ivraie Rieffel et 20 à $2 \breve{b}$ kilogrammes de grain d'ivraie Bailly. Le semis se fait de septembre à la fin d'octobre.

Ivrale d'Italie. - Vivace.

Stations. - Elle aime l'humidité, un sol frais, argileux, bien amendé, quoiqu'elle croisse aussi dans les terrains légers, pourvu qu'ils soient riches et humides. Elle craint les sols secs.

Propriétés. - Cette plante, qui produit considérablement dans certaines circonstances, donne quelquefois de faibles récoltes dans des terres qui sembleraient devoir lui convenir particulièrement. Tantôt elle fournit, pendant cing ou six ans, des récoltes abondantes; d'autres fois, malgré tous les soins possibles de culture, elle disparait subitement après la deuxième ou la troisième coupe, sans qu'on en connaisse la cause. Lorsque l'ivraie d'Ilalie se trouve sur un terrain où elle se plait, elle peut donner, moyennant des arrosements abondants, de trois à huit coupes par an, dont l'ensemble s'élève de 9,500 à 18,000 kilogrammes de foin à l'hectare.

L'ivraie d'Italie se sème seule ou en mélange: dans le premier cas, on peut faire le semis en auautomne ou au printemps, à raison de 40 kilogrammes; quand on le sème en mélange, on réduit la quantité, selon les circonstances. On peut lui associer le trèfle ordinaire ou le trèfle incarnat, dont s'est bien trouvé un cultivateur de notre pays, qui avait suivi les indications d'un agronome françass; il fit un mélange d'un tiers de graine de trèfle et de deux tiers d'ivraic d'Italie, et après 
l'enlèvement du trèfle, il conserva pendant quatre ans une prairie d'ivraie qui a donné des coupes abondantes et des plus succulentes.

Un autre essai où la graine de trèfle fut mêlée à de l'ivraie d'Italie, a fourni un résultat supérieur à celui qu'on avait obtenu par l'emploi du trèfle et de l'ivraie vivace. Tous les animaux, tant les vaches laitières que les boufs à l'engrais, s'en accommodent très-bien; elle pousse à l'engrais et augmente et améliore la sécrétion laiteuse.

Elle perd environ les deux tiers de son poids par la dessiccation.

M. De Dombasle, qui a étudié et expérimenté la culture de cetle plante sous toutes ses faces et dans les conditions les plus variées, s'exprime ainsi : "Il semble démontré, d'abord, que c'est seulement dans des sols de haute fécondité, et probablement dans des argiles calcaires, situées dans une position fraiche, que l'on peut espérer que cette plante se soutienne avec tout son luxe de végétation, et je dois regarder comme des exceptions mes récoltes de 1829 et 1830 . Il est probable que la beauté de ces récoltes, sur des terres blanches et graveleuses, a été due à ce que la semence avait été récoltée sur des terrains qui conviennent mieux à la nature de ces plantes; mais la semence produite dans des sols médiocres n'a plus donné de beaux produits que lorsqu'on l'a reportée dans des sols plus riches et d'une autre nature. Enfin, l'unanimité des observations faites en Suisse démontre que le purin ou engrais liquide convient d'une manière spéciale au Ray-Grass d'Italie; et l'on ne connaît aucune plante qui jouisse à un plus haut degré que celle-ci de la propriété de s'assimiler avec promptitude les principes nutri- 
tifs administrés sous cette forme, et de convertir en un très-court espace de temps, en nourriture pour le bétail, les urines des animaux et leurs excréments délayés sous forme de purin."

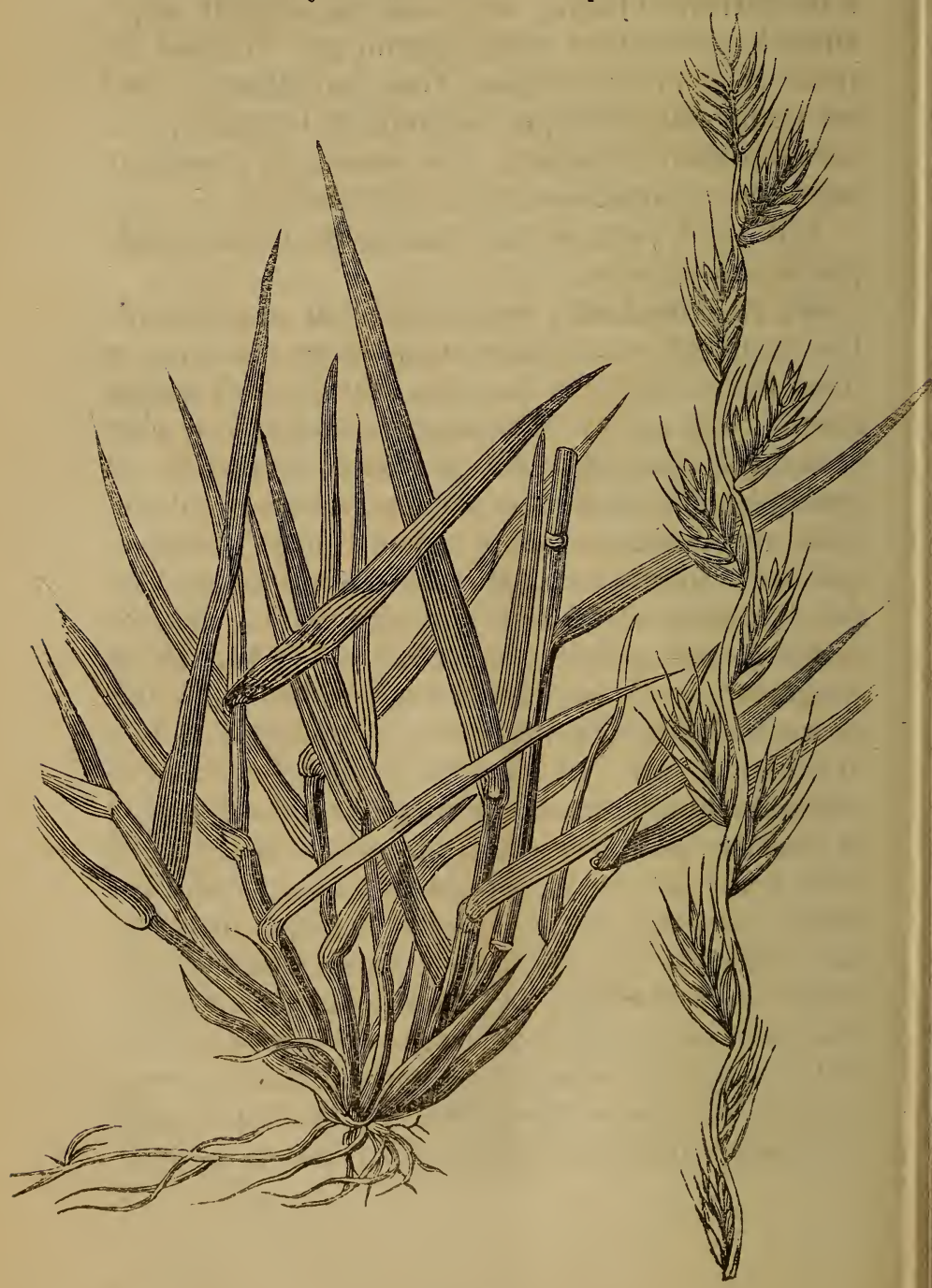

Fig. 67.

Irraic d'tlalic. 


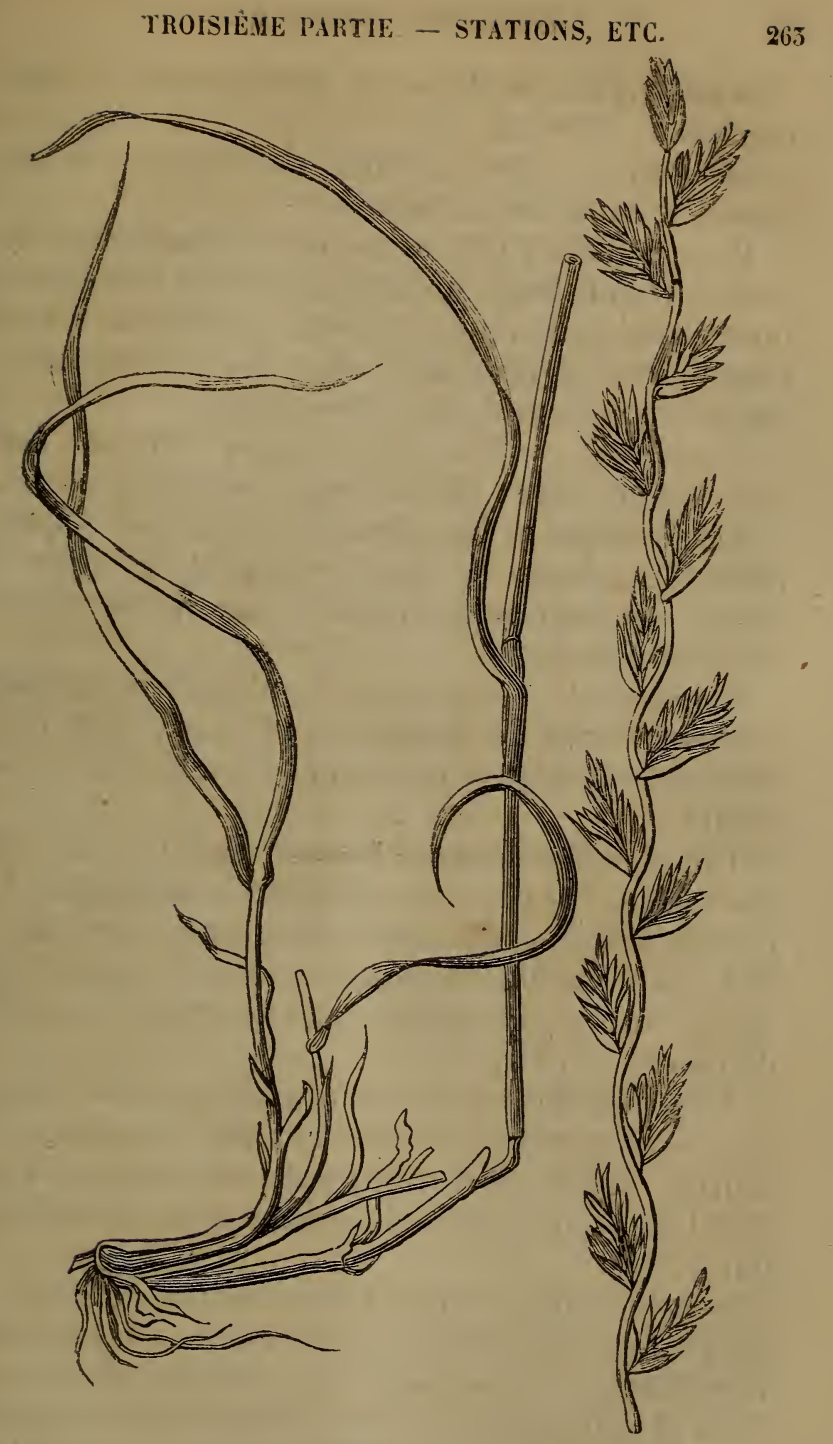

Fig. 68.

Irraie visace. 
Ivraie vivace. - Ray-gras d'Angleterre, gazon anglais. - Vivace.

Stations. - Prés, bords des champs et des fossés, le long des chemins.

Pour réussir, elle exige un sol gras, humide, assez fort, un loam, un sable argileux ; une constitution météorologique brumeuse et humide lui est singulièrement favorable : dans les terrains secs, légers et non fumés, elle reste chétive.

Propriétés. - Tous les animaux l'aiment, tant en vert que sec, et elle les engraisse promptement.

Les moutons ne la recherchent qu'avant l'apparition des chaumes; les chevaux et les bètes à cornes s'en montrent friands à tous les degrés de développement.

Rendement. - Lorsque cette plante très-précoce se trouve dans de bonnes conditions, elle peut donner trois coupes et fournir ensuite une bonne pâture.

Elle doit être coupée fréquemment; on la fauche lorsque les premières locustes se montrent. Le piétinement lui est nécessaire; aussi convient-il de la faire brouter de temps à autre, ce qui la fait taller, se ramifier et pousser une grande quantité de jeunes feuilles.

C'est une des meilleures graminées; elle donne une première coupe délicate et assez abondante, et fournit, après, d'excellents regains, lorsqu'on l'arrose largement et qu'on la submerge pendant quelque temps.

Semée scule, l'ivraie vivace donne pendant 2 à 10 ans de bon fourrage : nous la considérons comme la meilleure herbe pour les pâturages gras à sol frais et peu compacte ; c'est alors qu'elle procure le plus grand bénéfice. Les pâturages gras de Dix- 
mude et des environs sont en grande partie composés d'ivraie vivace et d'orge des prés.

Semée en mélange avec du trèfle ou de la lupuline, elle exige nécessairement un sol frais à soussol compacte. Cette prairie temporaire fournit une première coupe de fourrage, puis du pâturage pendant le reste de la saison.

Elle perd les deux tiers de son poids par la dessiccation ; et peut donner jusqu’a 3,750 kilogrammes de foin.

On emploie de 40 à 50 kilogrammes de semence par hectare de pâturage ou de prairie; pour la confection des gazons, on double cette quantité.

Lepiure arecé ét élancé. - Annuels.

Stations. - Lieux secs, sablonneux, dunes et sables maritimes.

Propriétés. - Ces plantes doivent ètre considérées comme des fourrages de médiocre qualité; d'ailleurs, elles sont peu productives. La dessiccation leur enlève la moitié environ de leur poids.

Nard serné. - Vivace.

Stations. - Lieux élevés, montagneux, secs, sablonneux et calcaires, rarement dans les terrains schisteux et les endroits marécageux : les terrains volcaniques sont ceux qu'il préfère.

Propriétés. - Cette plante est très-recherchée au printemps par les bêtes à cornes, mais elles ne touchent pas aux chaumes : elle est d'un grande utilité dans les endroits sablonneux où elle peut être pâturée.

Rendement. - Celle plante qui forme des touffes ëpaisses se montre très-difficile à couvrir un certain espace; par cela même, son rendement, lorsqu'elle est semée seule, ne peut avoir de l'importance. Une pelouse reg̣ulière peut donner 
MANUEL DES GRAMINÉES.

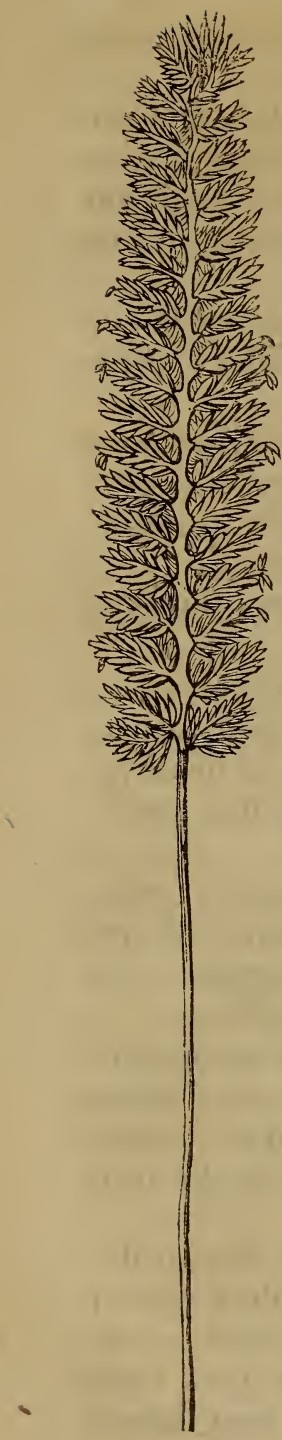

2,700 kilogrammes de foin. Elle perd les deux cinquièmes de son poids.

IIr tribu. - Eestucacées.

Gynosure cretelle. - Vivace.

Stations. - Dans tous les terrains sains où l'eau ne reste pas stagnante; prés et clairières des bois.

Propriétés. - Elle plaît à tous les bestiaux dans sa jeunesse et fournit un foin délicat qui indique des prairies favorables à l'hygiène du bétail : on lui attribue des qualités spéciales qui influeraient beaucoup sur la nature et le goût des fromages, mais on n'a pas encore constaté par la voie expérimentale jusqu'à quel point cette assertion est fondée.

Rendement. - Cultivée seule, cette plante donne un faible produit et perd les deux tiers de son poids par la dessiccation. Elle fournit 2,100 kilogrammes de foin.

Gynosure hérissé. - Annuel.

Stations. - Terrains humides chauds.

Propriétés. - Cette plante qui est recherchée dans sa jeunesse par tous les bestiaux, ne prospère pas bien en Belgique; d'ailleurs, son produit est peu abondant.

Fétuque $\Lambda$ UNe palleEtTe, fÉtuque DES SOURIS, FÉTUQUE QUEUE D'ÉCUREUIL.

Fig. 69.

- Annuelles. 
Cesplantes qui aiment les sols secs, sablonneux, et les décombres où elles se multiplient beaucoup, plaisent dans leur jeunesse à tous les ruminants ; mais comme ce sont des espèces peu productives et qui jaunissent de bonne heure et mème avant d'avoir fleuri, on doit les placer au dernier rang: elles sont d'ailleurs très-sujettes à la rouille.

Fétuque polynorpile. - Vivace.

Stations. - Lieux secs, élevés, sablonneux; coteaux pierreux, crayeux, siliceux.

Propriétés. - Celte espèce renferme deux races; la première présente deux variétés, dont l'une plaît aux moutons : c'est la fétuque ovine de Linné; l'autre est la fétuque à feuilles ténues de Sibthorp; ils ne la mangent qu'en hiver. Les autres ruminants la paissent volontiers. Son fourrage, tout en élant dur, est succulent et engraisse promptement les moutons.

Rendement. - Cette race formant des touffes épaisses, isolées, qui s'arrondissent et s'étendent successivement, n'est guère productive; cependant elle constitue une ressource précieuse pour les landes sablonneuses et siliceuses où elle peut ètre broutée pendant huit à dix ans.

Elle perd un peu moins des deux tiers de son poids par la dessiccation et peut donner $3,000 \mathrm{ki}-$ logrammes de foin.

M. Vilmorin qui a expérimenté la culture de cette plante seule et mêlée à d'autres, a remarqué que les troupeaux ne la pâturaient bien qu'en hiver et qu'en été les moutons ne mangeaient que les pieds isolés. Je l'emploie souvent en mélange, dit-il; mais j'en fais aussi des pièces séparées, à raison des ressources qu'elle offre pour l'hiver et de l'avantage qu'elle posséde de s'établir avec 
vigueur sur les terres arides, soit siliceuses, soit calcaires, et de les couvrir d'un gazon épais et durable. Quand on la sème seule, il faut environ 30 kilogrammes de grain à lhectare.

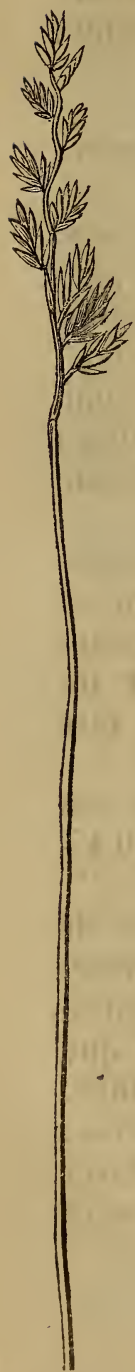

Fig. 70 .

Féluque à feuilies ténues.

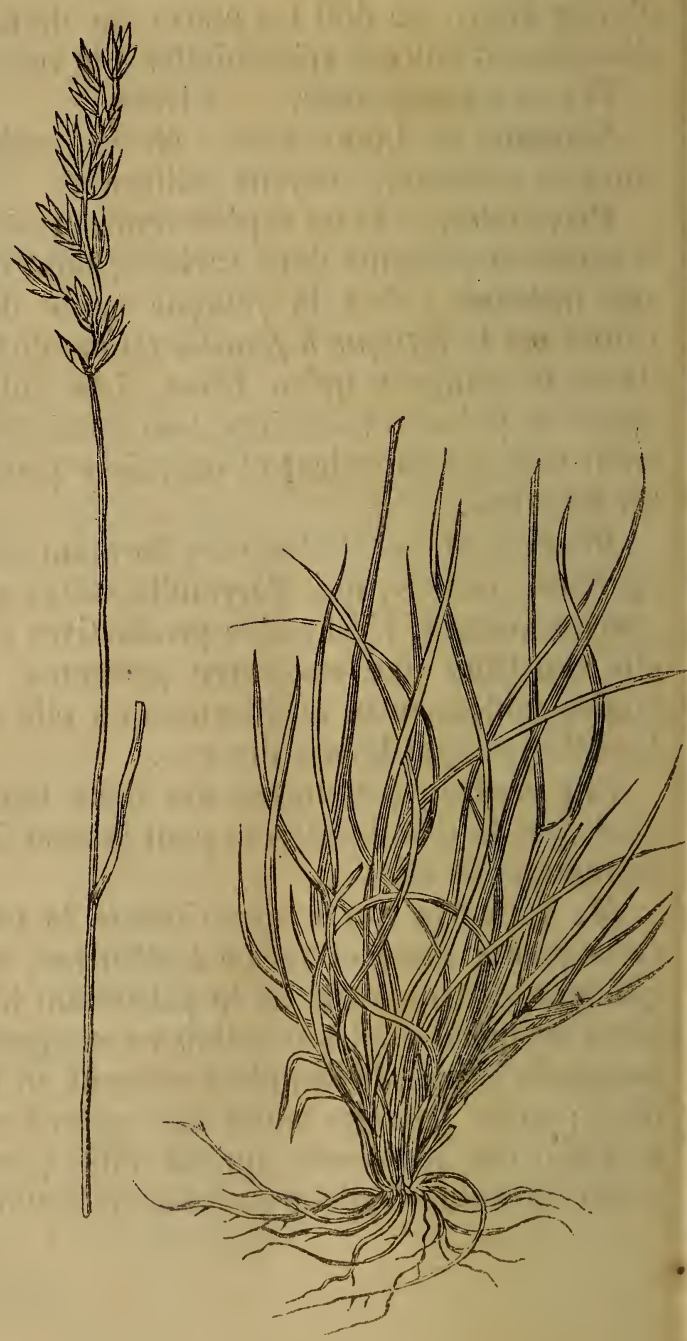

Fig. 71.

riluque ovine. 


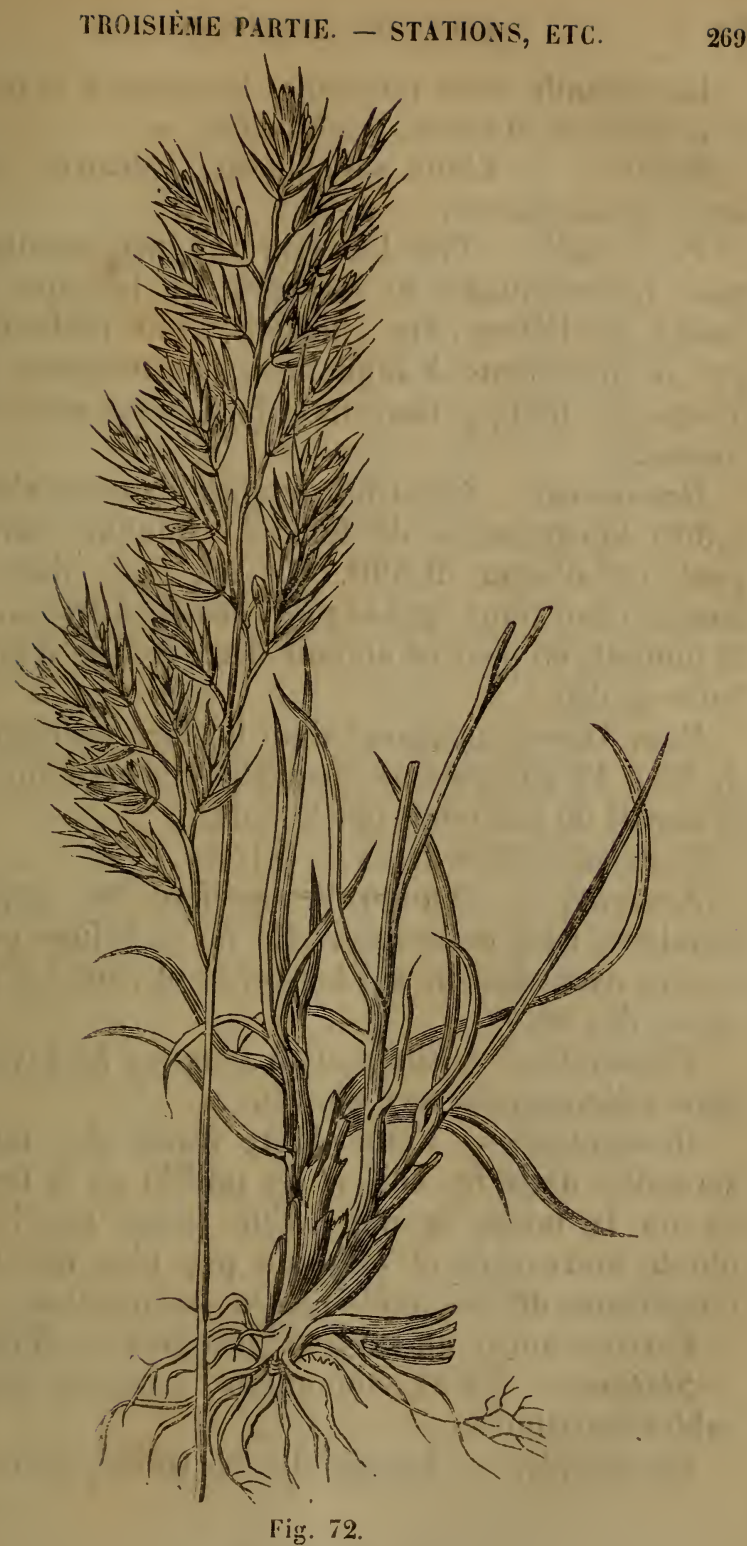

Fétuque durettle. 
La seconde race renferme la fétuque glauque et la fÉTuque durette. - Vivaces.

Stations. - Lieux sablonneux, calcaires, siliceux, montagneux.

Propriétés. - Très-bon fourrage, peu abondant, pour les ruminants et les rongeurs tels que les lapins, les lièvres, etc.; beaucoup plus recherchée que la précédente à laquelle ils ne touchent que lorsque la fétuque durette et la glauque sont consommées.

Rendement.-Celui de la fétuque glauques'élèveà 5,500 kilogrammes de foin; la fétuque durette peut en donner 9,300. On les sème dans les mêmes conditions que la précédente; l'arrosant et la fumant, on peut en obtenir deux coupes d'excellente qualité :

Pour l'ensemencement d'un hectare on emploie de 35 à 45 kilogrammes de grain ; elle perd environ la moitié de son poids par le fanage.

Fétuque hétérophyle. - Vivace.

Stations. - Quoiqu'elle préfère les terrains humides, bas, ombragés, elle ne se refuse pas à croitre avec vigueur sur les bords et dans les clairières des bois.

Propriétés. - Elle plaît à tous les herbivores, mais spécialement aux chevaux.

Rendement. - Cette plante forme des touffes arrondies dans les bois et les taillis; on la fauche ou on la laisse brouter. Elle forme une bonne plante fourragère et perd un peu plus des trois cinquièmes de son poids par la dessiccation.

Fétuque rouge ou fétuque traçante. - Vivace.

Stations. - Lieux sablonneux, siliceux, dunes, sables maritimes.

Propriétés. - Lorsqu'elle se trouve dans les 


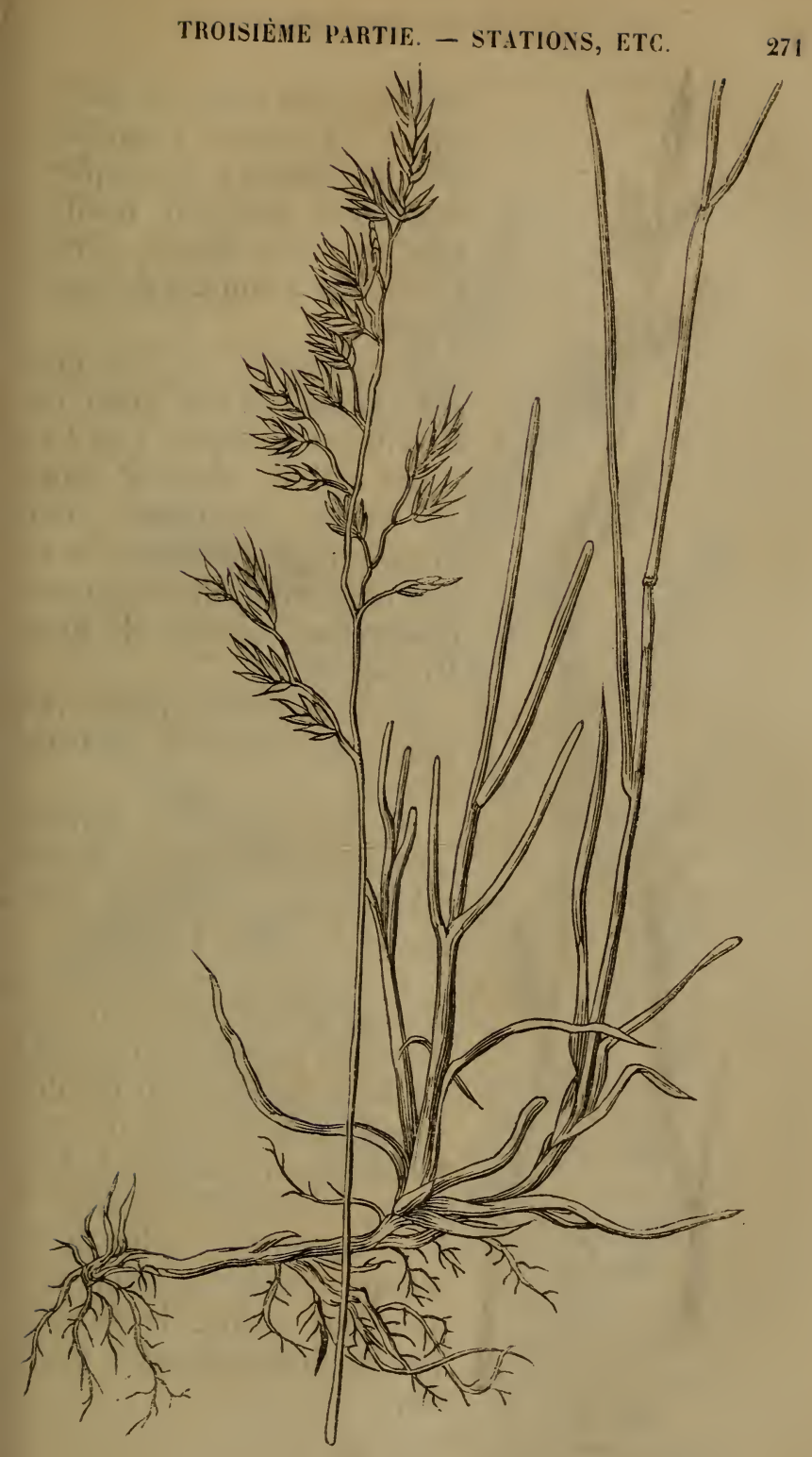

Fig. 75.

Fèturge ronge. 


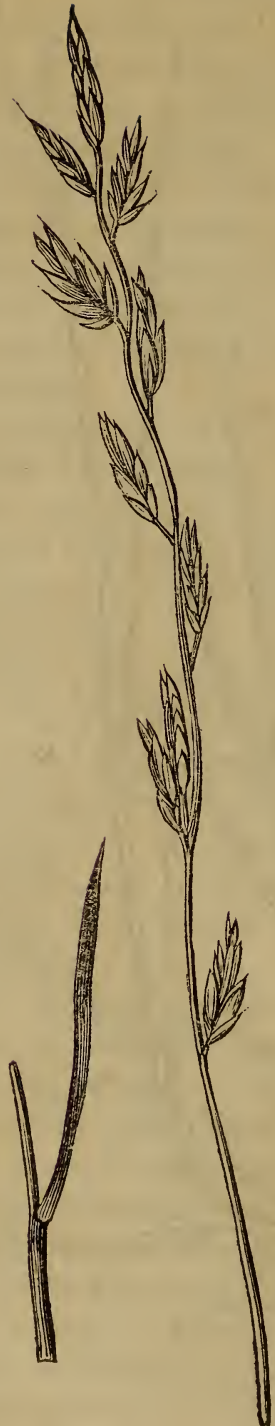

Fig. 74.

terrains secs susmentionnés, elle a les mêmes propriétés que la fétuque polymorphe; mais dans une terre fertile, elle peut être classée parmi les plantes à faucher debonne qualité.

Rendemient. - Un peu plus abondant que celui de la fétuque glauque. Les variélés qui se trourent dans les sables maritimes sont beaucoup plus productives et précoces; mais elles ont pour destination spéciale de fixer l'arène.

Fétuque fausse ivraie, féTUQUE DES PRÉS el FÉTUQUE roseau.

Stations. - Ces plantes aiment un sol loameux, ou un sable argileux frais et submergé de temps à autre.

Propriétés. - Elles fournissent un fourrage un peu grossier, composé en grande partie de fascicules de feuilles stériles, que les bestiaux consomment avec plaisir; le foin en est bon, facile à sécher et à se conserver : celui de la féluque ivraic est supéricur aux deux autres; la fétuque roseau fournit le plus grossier.

Felurgue fausse irraie. 


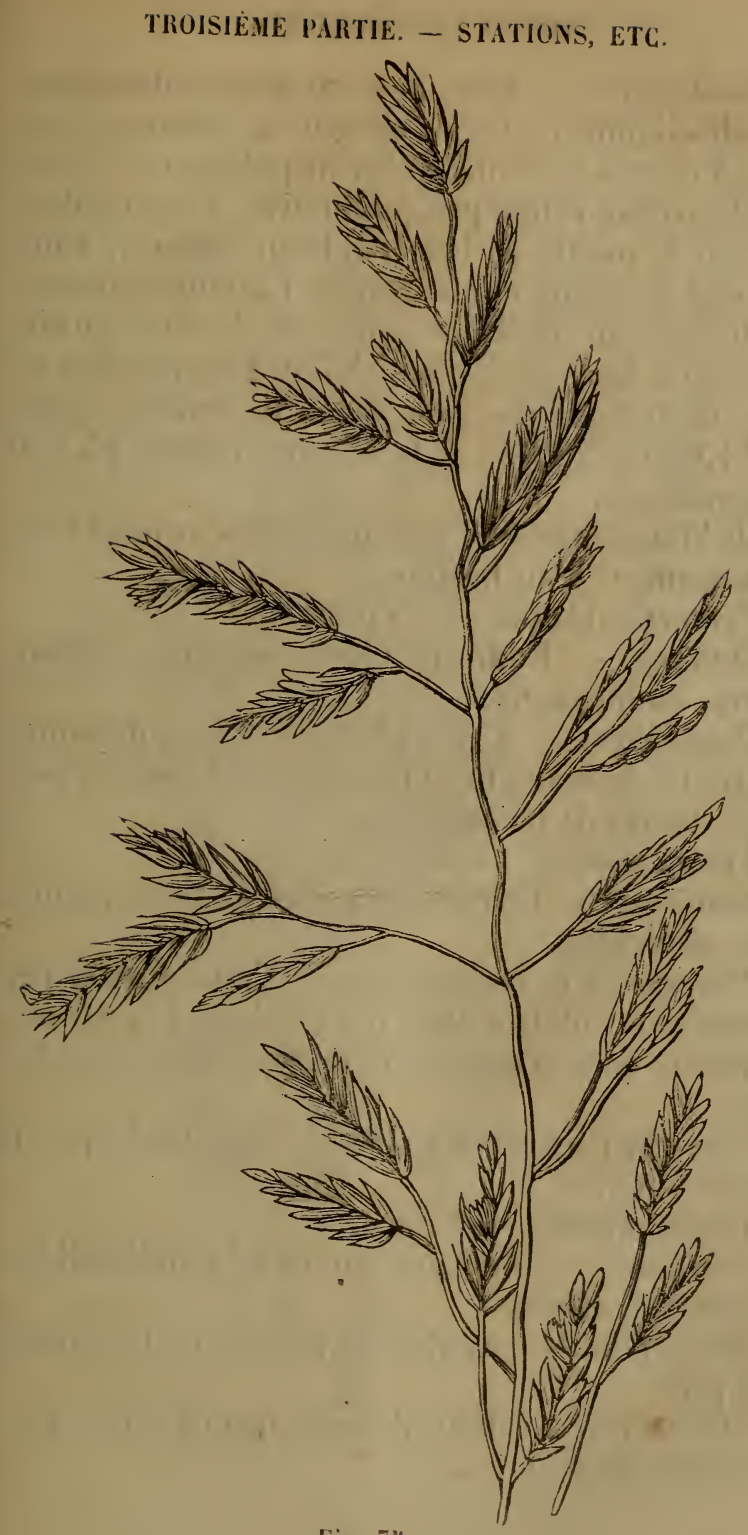

Fig. 73.

Fetuque des pres 
Rendement. - Elles peuvent donner deux coupes abondantes et servir ensuite de pàturage aux bêtes à cornes, à moins qu'on ne préfère en obtenir une troisième coupe peu productive. Elles perdent environ la moitié de leur poids en séchant. Elles donnent du regain en abondance. La fétuque fausseivraie devient d'autant plus productive qu'elle avance en âge; elle donne 14,350 kilogrammes de foin. La fétuque des prés, selon la variété, donne de 14,000 à 20,000 et la fétuque roseau 23,950 kilogrammes.

50 kilogrammes de semence suffisent pour l'ensemencement d'un hectare.

Fétuque calamaire. - Vivace.

Stations. - Endroits élevés, terrains calaminaires, montagneux.

Propriétés. - Cette plante se durcil de bonne heure; les bestiaux ne la mangent que dans les premiers temps de la végétation.

Fétuque GÉANt. - Vivace.

Stations. - Terrains humides de toute nature, mais ombragés.

Propriétés et rendement. - Cette plante, peu productive, plaît à tous les bestiaux et peut ètre convertie en foin avant le développement des chaumes.

Elle perd les trois quarts de son poids par la dessiccation.

Brome inerme. - Vivace.

Stations. - Terrains plutôt secs qu'lıumides, compactes, élevés.

Propriétés. - Analogues à ceux de la fétuque des prés.

Rendement. - Peut donner jusqu'à 6,000 kilagrammes de foin. 
TROISIEME PARTIE. - STATIONS, ETC.

Brome dressé. - Vivace.

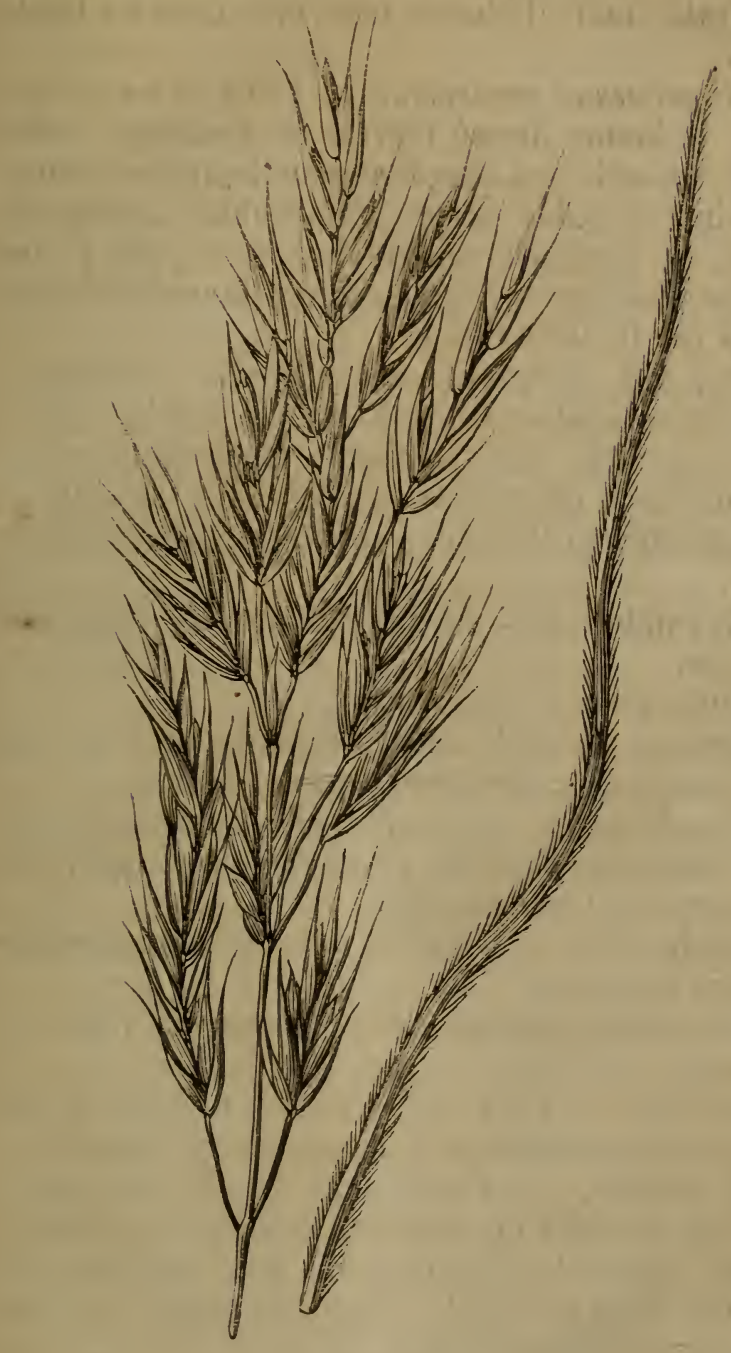

Fig. 76.

Brome dresse. 
Stations. - Il préfère les terrains loameux, un peu frais; mais il réussit aussi bien dans les hauts prés.

Propriétés et rendement. - Dans les terres fertiles, le brome dressé fournit un fourrage tendre et délicat, mais peu abondant; dans les terres sèches, calcaires et sablonneuses, il s'établit vigoureusement, eu égard à la qualité du sol, et peut y ètre fauché ou pâturé : il perd environ la moitié de son poids par la dessiccation.

On a fait quelques essais avec cette plante dans des terres médiocres où elle a été semée seule : elle y a donné deux coupes satisfaisantes et un regain tardif qui neétait pas dépourvu de mérite; son produit s'est élevé à 8,500 kilogrammes de foin.

On emploie 40 à $5 \breve{~ k i l o g r a m m e s ~ d e ~ g r a i n ~ p a r ~}$ hectare.

Brome rude. - Vivace.

Stations. - Sols un peu humides de toute nature, ombragés; dans les buissons, les bois.

Propriétés. - Les feuilles et les chaumes plaisent dans leur jeunesse : une fois développés, les chevaux n'y touchent plus.

Rendement. - Il monte à 4,500 kilogrammes de foin médiocre.

Brome roide, brome stérile et brome des toits. Annuels.

Stations. - Lieux secs, arides; prairies et pelouses sablonneuses; prés loameux, secs, élevés.

Propriétés. - Espèces fourragères médiocres que les bestiaux ne mangent qu'au premier printemps, avant la formation de leur panicule piquante. Elles perdent à peine la moitié de leur poids par la dessiccation. 
Brome squarreux, brome des Cilamps, brome de MICHEL, BROME ÉPAIS, BROME LUISANT, BROME SEIGLIN.

Toutes ces espèces qui sont annuelles et que l'on trouve dans les moissons et les prairies artificielles, fournissent un fourrage qui plait, lorsqu'elles sont jeunes, à tous les animaux herbivores: ils n'y touchent plus quand les chaumes ont acquis quelque dureié. Elles ne nuisent pas, néanmoins, aux produits des luzernes et des trèfles où elles pullulent parfois.

Quelques auteurs préconisent la culture du brome seiglin comme plante fourragère; nous croyons que cette recommandation ne repose sur aucun fait soigneusement observé et comparé avec d'autres fourrages graminées qui lui sont infiniment supéricurs sous le rapport de la qualité et de la quantité. L'orge, les ivraies et d'autres espèces analogues mériteront, dans tous les cas, la préférence, car le brome seiglin, pour donner un produit un peu important, exige une très-bonne terre bien fumée. On sera convaincu de l'exactitude de cette observation lorsqu'on aura parcouru quelques champs d'orge et qu'on aura comparé le nombre et le volume des jets de l'orge avec ceux du brome seiglin. Ensuite, la précocité, qui est un autre point non moins important, est aussi à l'avantage de l'orge commune. Le brome seiglin et ses congénères peurent fournir 15,000 kilogrammes de foin et plus.

BRome EN GRAPPE, BRONe ÉCHANGe el brone nou. - Ces espèces qui sont annuelles murissent à des époques différentes : le brome mon est le plus précoce, ensuite le brome échangé ; le plus tardif des trois est le brome en grappe. On les trouve dans les prairies, ordinairement le long des sen- 
tiers et les bords des fossés, ainsi que dans les endroits incultes et secs.

Tous les animaux les appètent avant l'apparition des chaumes; mais elles fournissent un fourrage peu abondant qui perd les deux tiers de son poids par la dessiccation.

Le brome mou, à cause de sa grande précocité, est une plante très-médiocre dans les prairies destinées à la fenaison. Dans les pàturages, les mouions le recherchent quand il est très-jeune.

Enodie bleue. - Vivace.

Stations. - Endroits humides, ombragés ; terrains argileux, glaiseux et argilo-sablonneux, inondés pendant l'hiver.

Propriétés. - Tous les ruminants et les petits animaux domestiques, comme les lapins, etc., en aiment les fascicules de feuilles avant la floraison, et n'y touchent plus lorsque la panicule est développée; les oiseaux de basse-cour se montrent friands de son grain, à la chair desquels il communique un fumet agréable.

Rendement. - L'enodie bleue ne donne qu'un très-faible produit qui n'en autorise pas la culture. 2,000 à 2,500 kilogrammes de foin.

Elle perd un peu plus de la moitié de son poids par la dessiccation.

Brize a Gros ÉPILlets, BRIZE MOYenNe et BRIZE NaINE. - De ces trois espèces de brizes qui sont également agréables à la vue dans les jardins et les prairies, il n'en est qu'une qui intéresse le cultivateur : c'est la brize moyenne, qui s'accommode de tous les terrains, mais de préférence des sols graveleux bien aérés. Ses feuilles courtes en font une plante de pâture très-recherchée par les moutons; aussi la sème-t-on avec avantage, mêlée à la 
TROISIÈE P.IRTIE. - STATIONS, ETC.

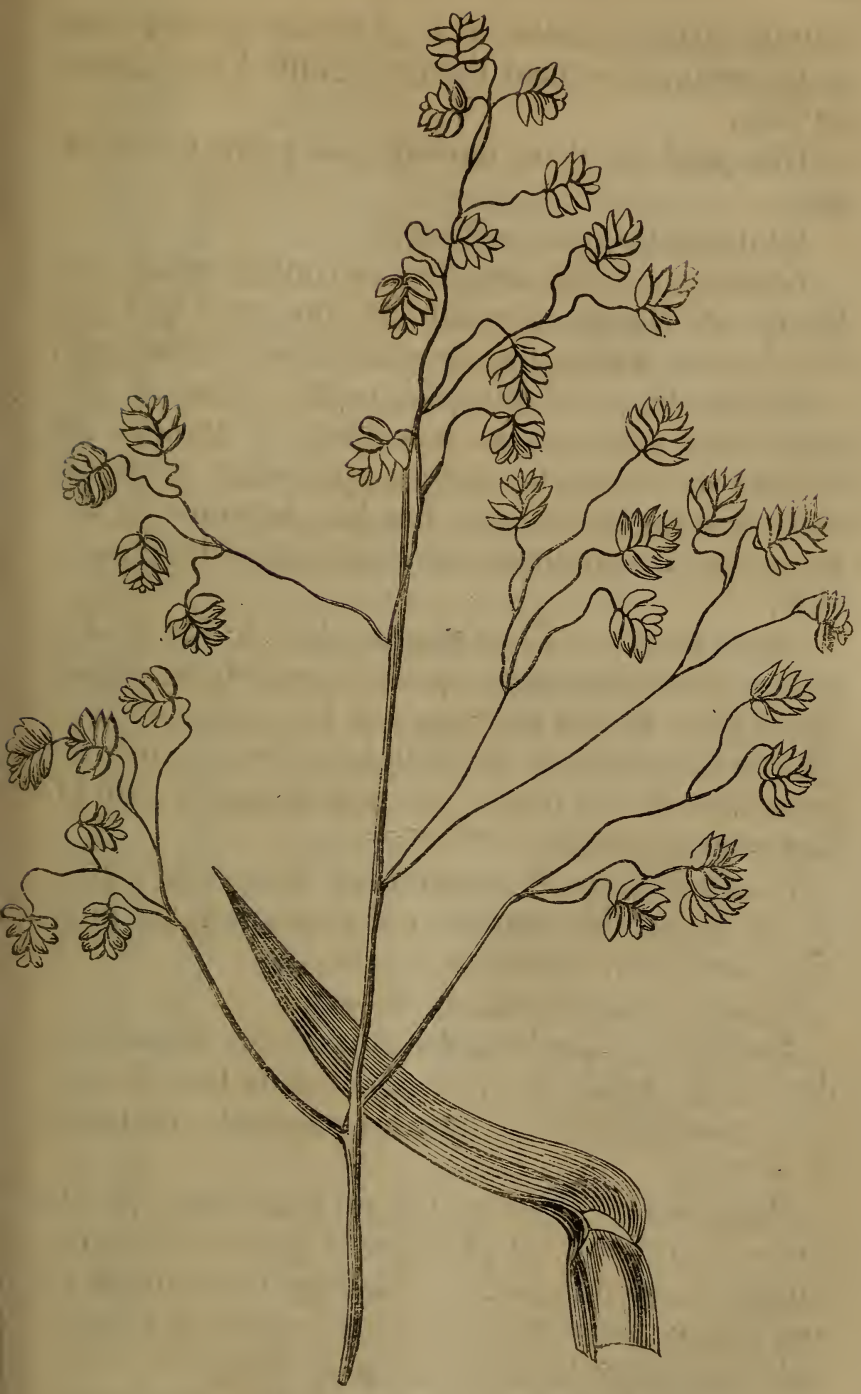

Fig. 77.

Erize moyenne. 
fétuque ovine, durette, etc., dans les terrains secs et pierreux. Elle peut donner $3, \mathfrak{5 0 0 0}$ kilogrammes de foin.

Elle perd les deux tiers de son poids par le fanage.

GLycÉrie élevéE. - Vivace.

Stations. - Elle aime les endroits humides, les fossés, les étangs, et vient partout, quel que soit le sol, pour autant que sa souche soit submergée.

Propriétés. - Cette plante plait dans sa jeunesse aux chevaux et aux bêtes à cornes; ses feuilles ses rejets stériles durcissent promptement et demandent à être fauchés de bonne heure, avant que les chaumes aient développé leur panicule.

Rendement. - Cetle plante, dans les terres humides et marécageuses, peut fournir deux coupes abondantes de bon fourrage que l'on donne en vert, ou que l'on fane; la fenaison lui enlève environ les deux tiers de son poids : elle peut donner 8,600 kilogrammes de foin.

En Angleterre, et notamment dans l'île d'Ely, on en forme des prairies qui produisent un fourräge abondant, tendre et succulent.

Glycérie flottante. - Vivace.

Stations. - Le long des ruisseaux, des mares, des fossés, bords des rivières et dans tous les terrains marécageux ou inondés pendant une partie de l'année.

Propriétés. - Il n'est pas de graminées que les chevaux recherchent plus que la glycérie flottante. Aussi ses feuilles et ses chaumes contiennent-ils une grande quantité des matières sucrée qui transsurleni pendant les jours les plus chands et se déposent sons la forme de taches et de stries brunà- 
TROISIĖIE PARTIE. - STATIONS, ETC.

tres à la surface du chaume vers le sommet et sur les divisions de la panicule.

Rendement. - Elle fournit un fourrage abondant, tendre et succulent, que l'on donne en vert ou en sec. Elle perd environ les trois quarts de son poids par la dessiccation et donne 5,600 kilogrammes de foin.

Le grain de cette espèce est trèsrecherché par les oiseaux aquatiques et les poissons; on prétend même que les carpes et les grenouilles se réunissent partout où la glycérie flottante se trouve en abondance.

Dans certains pays, et notamment en Pologne, on destine ce grain à divers usages économiques, et on en fait du gruau qui est trèsestimé.

La récolte de la semence se fait à la fin de l'été, en frappant avec des baguettes les locustes au-dessus d'un tamis.

On la multiplie au moyen du semis ou de fragments de rhizomes; le semis se fait très-clair, à cause de nombreux rhizomes qu'elle émet dès la première année.

Glycérie distante el glycérie m.ariTIIIE. - Vivace.

Stations. - Dans les sables maritimes et dans les prés sablonneux qui sont inondés de temps à autre par les Fig. 78.
cérie flotante. caux de la mer, et dans l'intérieur du 
pays où il existe des sources d'eaux minérales salines.

Propriétés. - Ces espèces sont recherchées par tous les bestiaux et donnent une grande quantité de fourrage excellent pour être consommé en vert; mais elles ne conviennent guère à être fanées, à cause des difficultés que l'on éprouve dans leur dessiccation. Elles perdent environ les deux tiers de leur poids par la dessiccation et peuvent donner ๖, 500 kilogrammes de foin.

SclérochloÉ covché. - Annuel.

Stations. - Lieux sablonneux inondés par les eaux de la mer, prairies des polders maritimes.

Propriétés. - Plaît à tous les bestiaux et est en quelque sorte l'analogue du paturin annuel.

Rendement. - Produit peu de fourrage et perd les deux tiers de son poids par le fanage, qui s'en opère assez difficilement.

Slérochló́ roide. - Cette plante, qui est annuelle, aime les endroits secs, sablonneux et calcaires, mais n'est guère recherchée par les bestiaux à cause de sa dureté.

Aelbroeckie narituae. - Mème importance que la glycérie maritime.

Dactyle pelotonné. - Vivace.

Stations. - Croît partout, mais recherche les terrains frais, substantiels, un peu ombragés.

Propriétés. - Les bœufs mangent volontiers cette plante jusqu'à l'époque 'dé la maturité des grains ; les chevaux et lesmoutons en aiment la pâture avant le durcissement des chaumes. C'est à la fois la plus précoce et la plus tardive de toutes les graminées vivaces. Les chiens la recherchent pour se faire vomir.

Rendement.-Par sa vigueur et sa rapide crois- 
sance, le dactyle étouffe souvent les espèces plus faibles qui l'entourent; il convient plutôt pour être consommé en vert et pâturé qu'à être converti en foin. Si on le destine à ce dernier usage, on doit le couper fréquemment, car ses feuilles et ses chaumes acquièrent bientôt assez de dureté pour déplaire au bétail. Quand il est semé seul, ce qui n'est pas à recommander, on doit le faucher près du sol; il fournit trois coupes et perd un peu plus de la moitie de son poids par la fenaison. Son produit en foin s'élève à 14,000 kilogrammes, celui du regain à 5,400 kilogrammes.

Il mûrit en juillet, et se sème au printemps ou en automne, à raison de 30 à 40 kilogrammes à l'hectare.

Le paturin annuel, dont le tableau a été retracé avec tant de vérité par Poiret, est celte graminée commune que l'on rencontre partout, dans les terrains incultes ou cultivés, dans les villes, les villages, le long des routes, dans les rues peu fréquentées, entre les pavés des cours, qu'il est d'ailleur's si difficile de détruire, qui ne cesse de se multiplier, quoique piétinée, broulée, arrachée; elle ne craint ni les froids du nord, ni les chaleurs du midi; elle forme des touffes très-étendues, fleurit et fructifie en tout temps, mème dans l'hiver, lorsqu'il ne gèle pas; elle offre le spectacle intéressant de la végétation luttant contre l'intempérie des saisons, contre les efforts de l'homme pour la détruire, lorsqu'elle cesse de lui être utile; elle couvre, en peu de temps, d'une belle verdure, les sols stériles et abandonnés. Si les longues sécheresses l'altèrent, les moindres pluies la raniment ; si les neiges la recouvrent après leur fonte, elle reparaît au milieu des frimas comme une tenture qui mas- 
TROISIÉME PARTIE. - STATIONS, ETC.

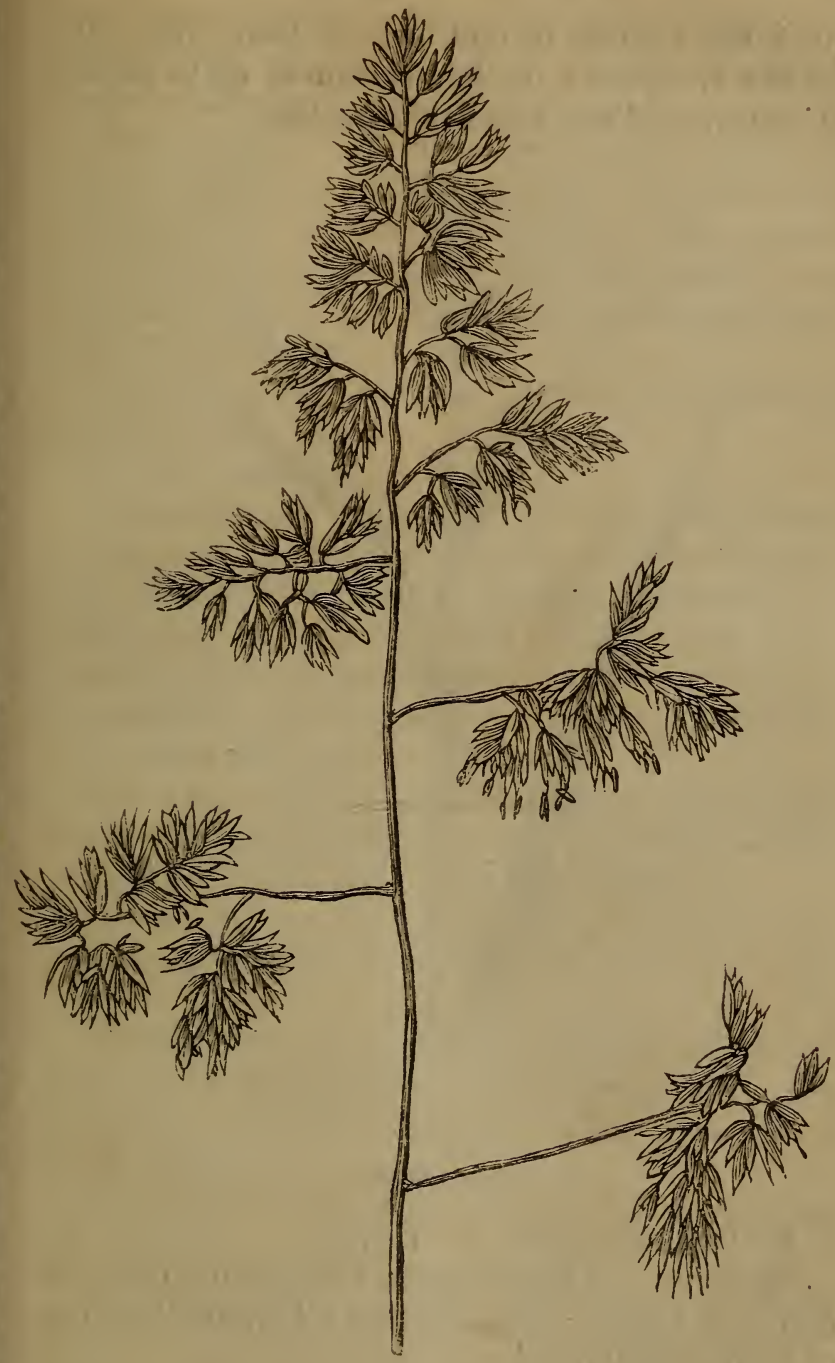

Fig. 80

Dactyle pelotonne. 
que à nos regards la nudité de la terre. Elle fournit aux troupeaux, malgré la rigueur de la saison, un pâturage d'une excellente qualité.

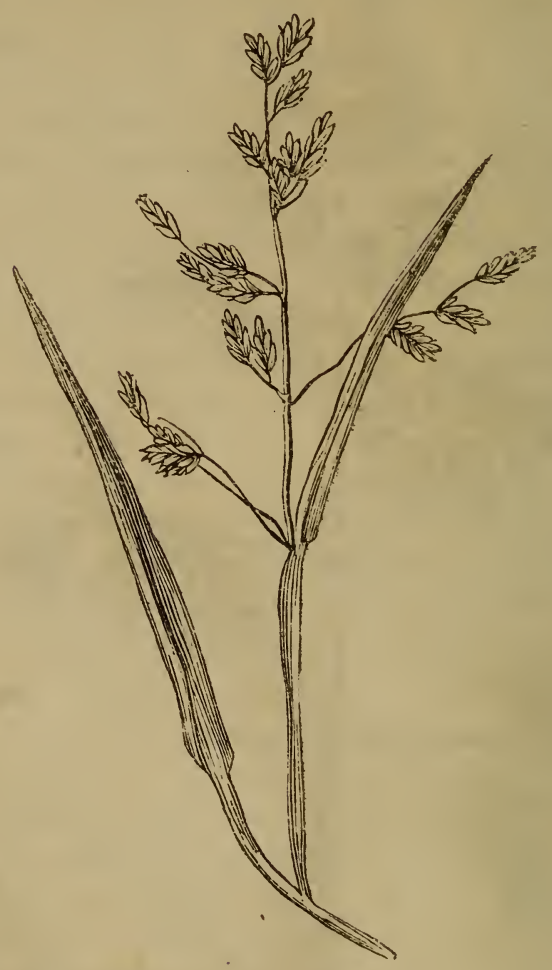

Fig. 81.

Paturin annuel.

Paturin des Alpes. - Vivace.

Stations. - Terrains secs, sablonneux, calcaires élevés; il réussit aussi dans un sol fertile, loameux el argilo-sablonneux.

Propriétés. - Cette plante est très-recherchée 
par tous les bestiaux; le lait des vaches et celui des chèvies en acquièrent des qualités butyreuses remarquables et un bon goût. Précoce.

Rendement. - II forme des touffes denses de feuilles assez longues, trop courtes cependant pour convenir dans les prairies fauchées, dont il pourrait tout au plus former le fond ; par contre, c'est une des herbes les plus propres pour les pâturages gras et les pacages.

Il perd environ les deux tiers de son poids par la dessiccation.

Paturin buibeux. - Vivace.

Cette espèce se trouve dans les lieux sablonneux, calcaires, arides, sur les vieux murs et dans les graviers d'alluvion: elle forme des touffes isolées que le bétail aime beaucoup; mais à cause de cette particularité, elle ne convient que pour les pâturages et les pacages : elle est d'ailleurs très-peu productive.

Paturin des bols. - Vivace.

Stations. - Lieux secs, ombragés; sols de toute nature, mais de préférence dans les terrains argilosablonneux et loameux, non submergés.

oropriétés. - Il est très-précoce et recherché par tous les besliaux, auxqeuls il fournit un fourrage excellent.

Rendement. - Cette plante, qui n'est pas gazonnante, perd les deux tiers de son poids par la dessiccation; on peut évaluer le produit en foin à 4,000 kilogr.

On emploie de 20 à $2 \breve{2}$ kilogrammes de semence par hectare.

Paturin fertile. - Celte espèce qui est vivace et qui aété considérée comme une variété de la précédente, aime les lieux humides des bois, forme des touffes assez serrẻes, et fournit un fourrage délicat 
MANUEL DES GRAMINÉES.

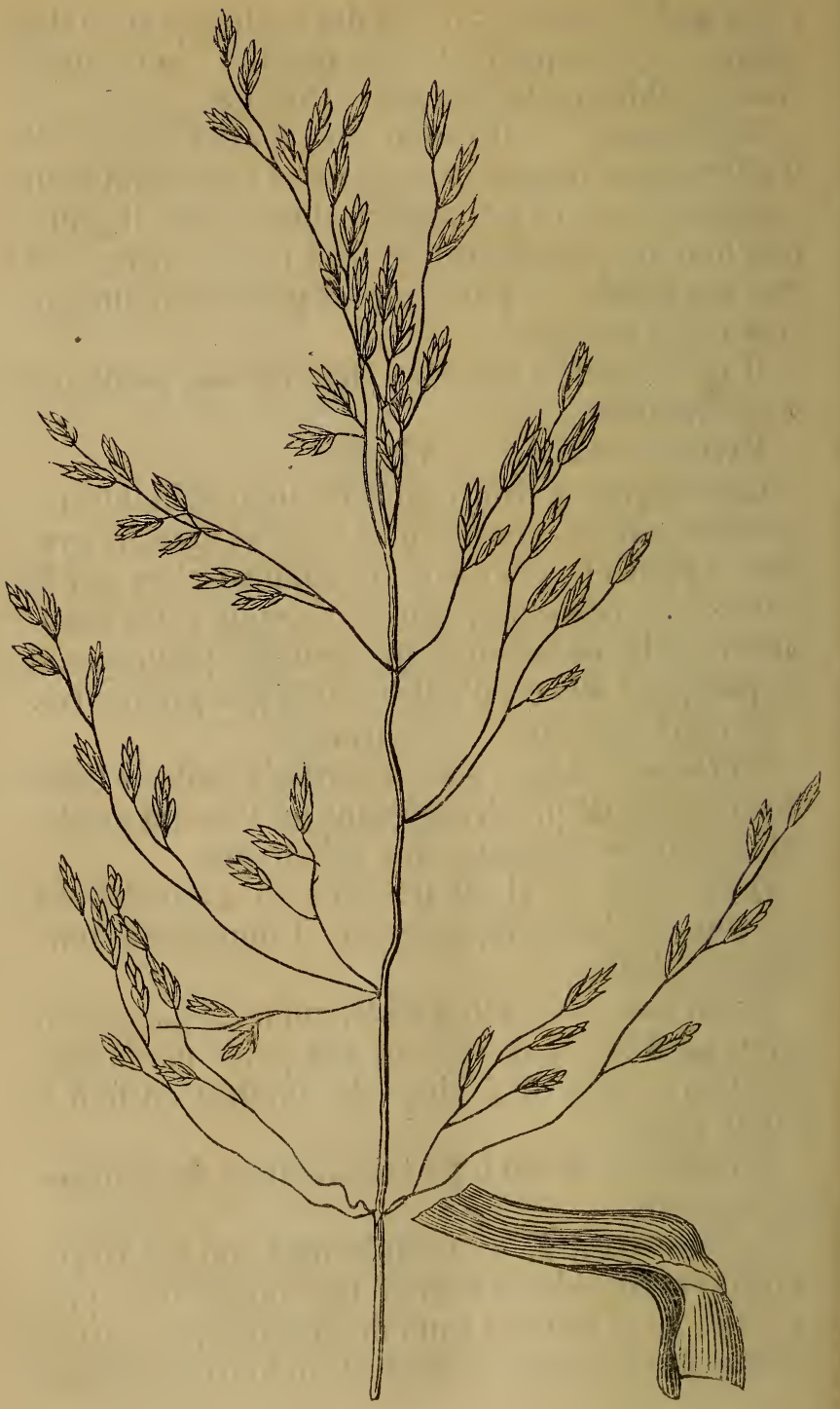

Fig. 82

raturin des bois. 
TROISIENE PARTIE. - STATIONS, ETC.

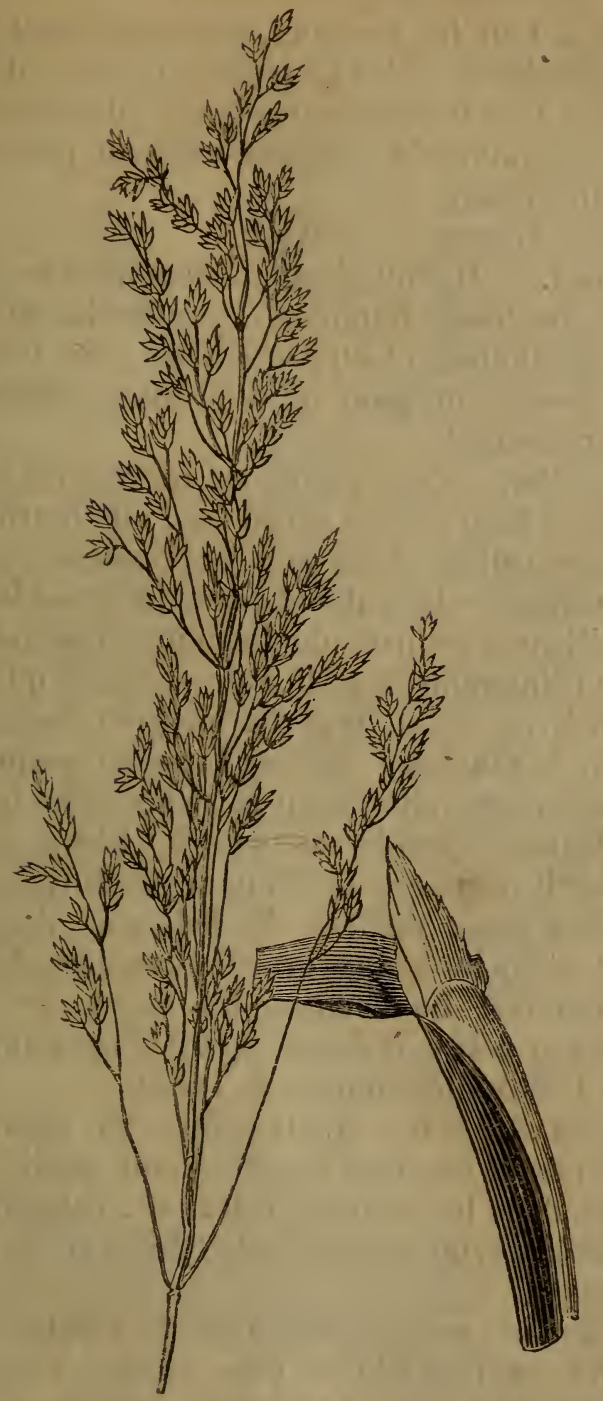

Fig. 83.

Paturiu fertile. 
qui plail à tous les bestiaux. Son rendement est à peu près double de celui du paturin des bois ;il perd environ les deux tiers de son poids par la dessiccation.

20 kilogrammes de semence suffisent pour emblaver un hectare.

Paturin commun. - Vivace.

Stations. - Il croît abondamment dans les prés, les haies, les fossés humides, et préfère les terrains frais, substantiels et un peu abrités : les terrains secs et froids sont pour lui des causes nécessaires de dépérissement.

Propriétés.-Cette plante est recherchée par le bétail aussi bien en vert qu'en sec; elle fournit un fourrage excellent et un foin de première qualité.

Rendement. - Le paturin commun forme la base des meilleures prairies de Belgique; comme il se trouve ordinairement mêlé à des espèces qui sont plus tardives, si on veut l'avoir arec toutes ses qualités, le fauchage doit se faire à l'époque de la floraison, car elle jaunit et se dessèche bientôt après. Dans les prés du comté de Willshire, en Angleterre, il acquiert la hauteur gigantesque de vingt-deux pieds anglais. En Belgique, il mesure souvent, dans les bonnes prairies irriguées, de 11 à 19 décimètres. La fenaison lui enlève les deux tiers de son poids; il donne 2,600 kilogrammes de foin et 1,600 kilogrammes de regain.

Paturin bleuatre. - Cette plante, qui est vivace, fournit peu de fourrage de très-bonne qualité; elle convient dans les terrains calcaires, calaminaires et sablonneux, où elle est recherchée par les petits ruminants.

Paturin des prés. - Celte espèce a beaucoup de rapports, sauf qu'elle est plus précoce encore de quelques jours, avec le paturin commun. Comme 
TROISIÉIIE PARTIE. - STATIONS, ETC.

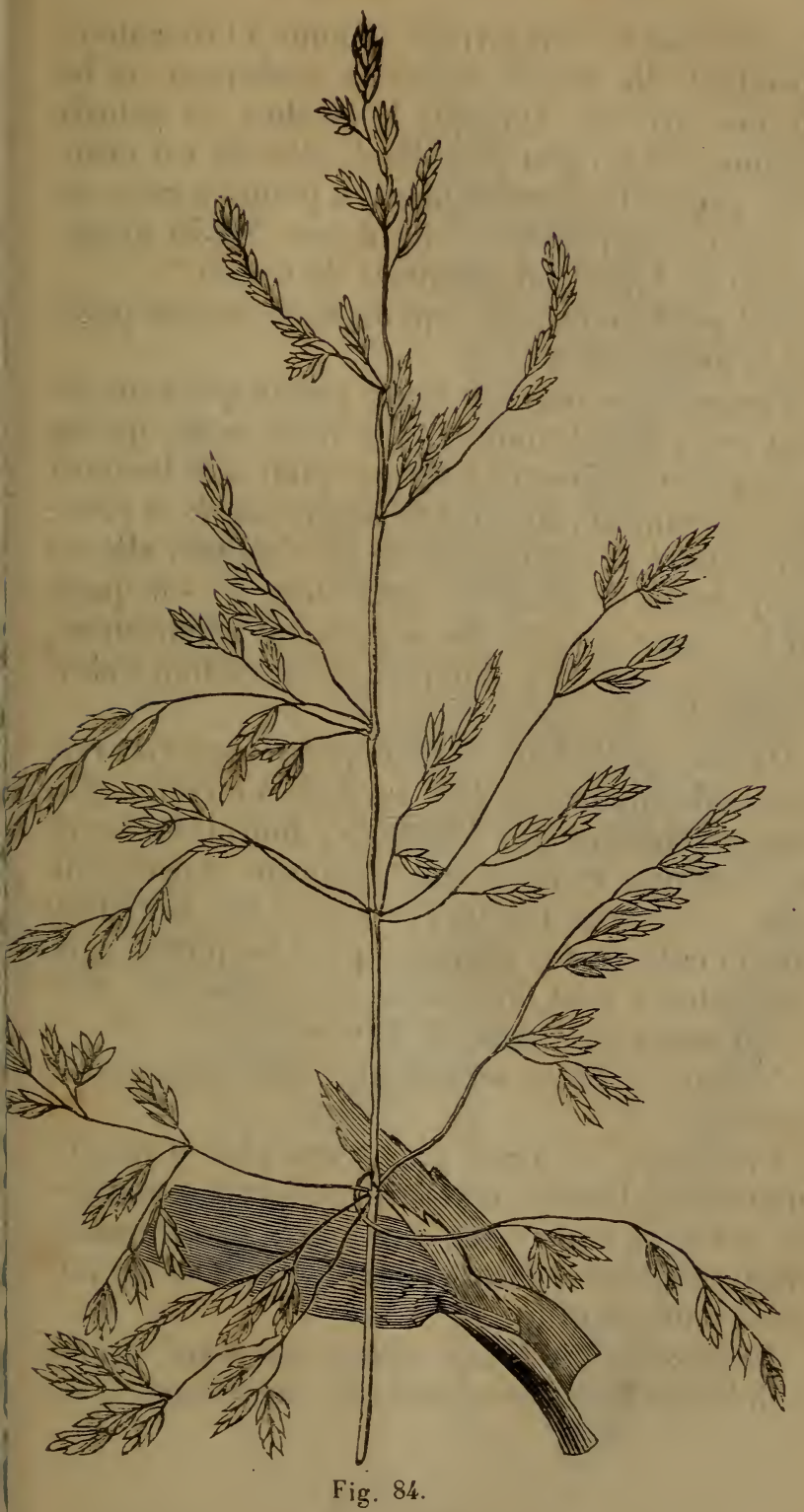

Palurin commun. 
lui, elle aime les sols fertiles, soumis à l'irrigation; cependant elle craint moins la sécheresse et les sols non arrosés. Quoique le produit du paturin commun soit un peu plus élevé, elle lui est néanmoins préférable comme plante à pâture à cause de sa prompte repousse : elle donne $3,250 \mathrm{kilogr}$. de foin et 1,380 kilogrammes de regain.

Elle perd environ les sept dixièmes de son poids par la dessiccation.

Paturin comprimé. - Cette plante qui aime les lieux secs, les décombres, les vieux murs qu'elle perce par ses rhizomes traçants, plaît aux bestiaux dans sa jeunesse, mais est dédaignée après, à cause de la dureté de ses tiges et de ses feuilles; elle est très-précoce et perd les deux tiers de son poids par le fanage, auquel elle ne convient aucunement, quoi qu'on en ait dit. Son rendement en foin s'élève à $1,6 \breve{0} 0$ kilogramınes.

Paturin de Silésie. - Cette plante que l'on rencontre abondamment dans les bois à terrains glaiseux et schisteux des Ardennes, fournit un excellent fourrage et en grande quantité. Cependant, nous ne croyons pas qu'elle puisse être introduite dans la culture des prairies, pour lesquelles quelques auteurs l'ont fortement préconisée.

Catabrose aquatique. - Vivace.

Stations.-Lieux aquatiques, ombragés; marais tourbeux.

Propriétés. - Cette plante qui plaît à tous les animaux herbivores quand elle est fauchée, est rebutée après sa conversion en foin : elle repousse très-promptement lorsqu'elle a été broutée ou fauchée en temps utile.

Rendement. - Celte espèce est d'une grande utilité dans les terrains humides, marécageux; son 
produit, qui n'est pas très-élevé (on l'évalue ì 5,675 kilogrammes), perd environ les quatre cinquièmes de son poids par la dessiccation; on la propage comme la glycérie flottante.

Eragrostide poilue, eragrostide vulgaire. - Ces espèces qui sont annuelles, et non vivaces, comme le dit à tort Lecoq, aiment les terrains secs, sablonneux; elles sont assez recherchées par le bétail, mais leur produit est insignifiant.

Roseau a balars. - Vivace.

Stations. - Cette plante est commune le Iong des fossés, des marais et des étangs, et dans les prés tourbeux.

Propriétés. - Elle plaît aux bœufs Iorsqu'elle est jeune et avant que ses feuilles aient pris la consistance qui les distingue; on pense, et quelques agronomes prétendent que ce fourrage qui contient une quantité notable de matière sucrée, augmente considérablement la sécrétion laiteuse et communique au beurre et au fromage qui en proviennent des qualités supérieures.

Rendement. - Elle produit considérablement, mais elle doit être fauchée jeune pour plaire aux bestiaux : coupée après son développement, ils la refusent. Son rendement monte à 20,000 kilogrammes de foin.

Mélique ciliéE. - La précocité est le principal caractère utile de cette plante ; elle est très-recherchée par les bestiaux, mais son mode de croître en touffes serrées est contraire à une culture régulière.

Mélique penciée et mélique uniflore. - Vivaces.

Stations. - Bois montagneux et lieux ombragés.

Propriétés. - Les auteurs ne sont pas d'accord sur les propriétés de ces espèces: les uns croient 


\section{TROISIEME PARTLE. - STATIONS, ETC. 293}

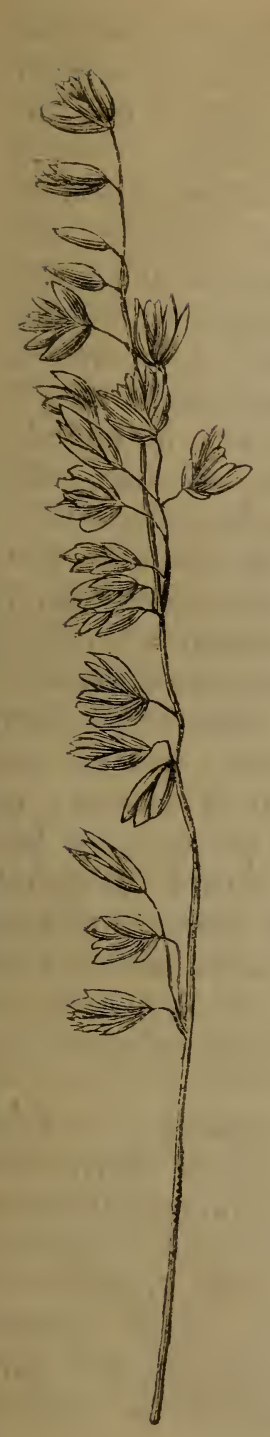

Fig. 86 .

Melique penchee.

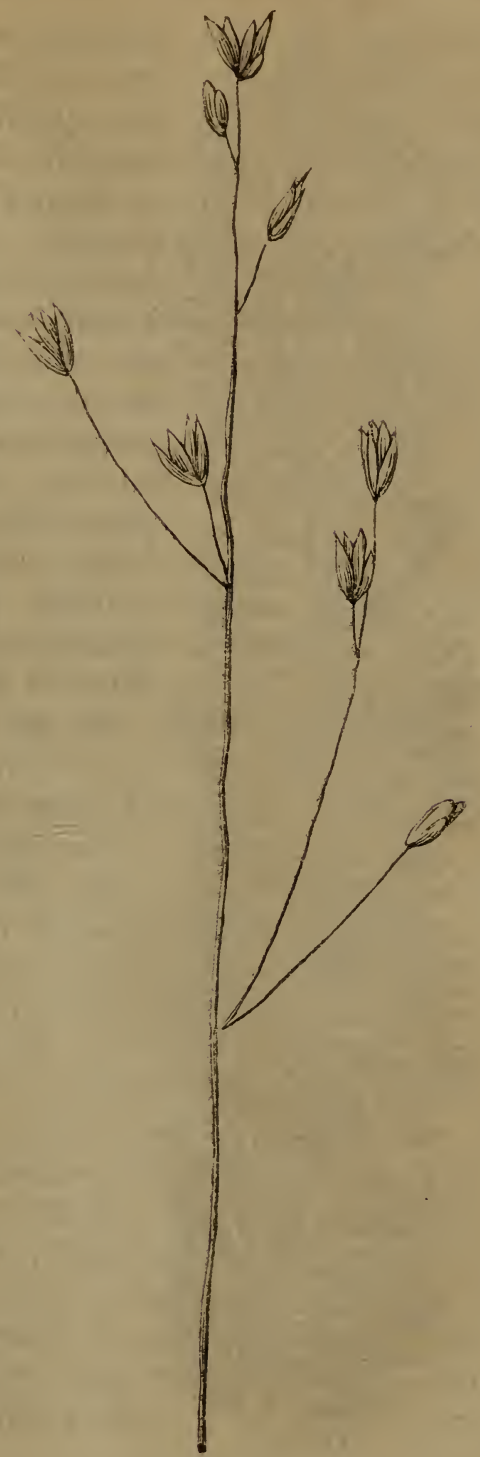

Fig. 87.

Meligue uniflore. 
MANUEL DES GRAMINÉES.

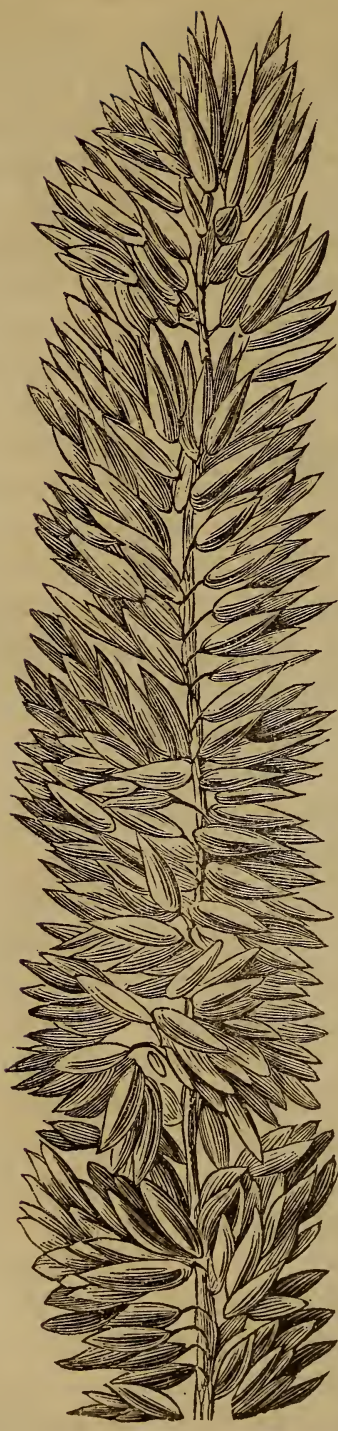

Fig. 88.

qu'elles plaisent à tous les bestiaux; les autres soutiennent que la plupart des animaux les refusent, et que si les chevaux les mangent quelquefois, c'est toujours avec un dégoût très-marqué. Quant à nous, nous pouvons assurer que nous les avons données à des chevaux et qu'ils n'en témoignèrent pas le moindre dégoût; au contraire, elles paraissaient leur plaire beaucoup; aussi pensons - nous qu'elles pourraient servir avantageusement à garnir le fond des bois secs et élevés.

Mélique Élevée. - Cette espèce vivace, qui est peu délicate sur le terrain, est recherchée par tous les bestiaux. M. Ivart dit qu'elle fournit un fourrage précoce d'une excellente qualité. Toutefois, il faut avoir soin de la couper avant qu'elle se soit durcie.

Triodie pencuée. - Cette espèce vivace se trouve dans les lieux ombragés, les boistaillis, les landes stériles, les prés secs, argileux et sablonneux. Cette plante, qui ne convient que pour le pâturage et la pacage, Mélique élevéc. 
constitue un bon fourrage, mais peu abondant.

Koelerie crêtée. - Celle plante aime les en- hool. crusten droits secs, sablonneux, calcaires, les dunes et tous les sols volcaniques : elle plait à tous les bestiaux, repousse facilement et fait, à juste titre, partic des meilleures pâlures sèches. Lorsque la souche commence à vieillir, elle forme des touffes grosses et saillantes auxquelles les animaux ne touchent qu'au printemps. Elle perd environ la moitié de son poids

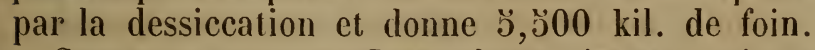

Seslérie bleue. - Celle plante vivace, qui est Ses? cuermiea des plus précoces, est commune dans les lieux montagneux où elle prospère dans les terrains maigres et rocailleux, pour autant qu'elle y trouve un peu d'humidité. Sa précocité la rend précieuse dans les localités montagneuses où les moutons peuvent la brouter immédiatement après la fonte des neiges : elle n'est pas propre au fauchage.

\section{Ive tribu. - Aénacées.}

Conynéphore blaxchitre. - Cette petite plante vivace, qui aime les lieux secs, sablonneux, fournit un fourrage délicat, mais en très-petite quantité.

Avorne NCE. - La grosse avoine nue qui fournit $\hat{A}_{2}$ ena muda un excellent gruau est beaucoup plus productive que la petite; mais elle demande une terre fertile.

Avorne cultivée. - L'avoine cultivée a fourni un Ar. saléra grand nombre de variétés qui s'accommodent les unes mieux que les autres des sols médiocres; celles qui fournissent le plus grand rendement et de meilleure qualité sont, dans les terres fertiles : l'avoine patate (malheureusement assez sujette au charbon), l'avoine blanche des Flandres, l'avoine de Kamschatkn, l'avoine de Brie. Dans les 
terres moins fertiles, on peut encore cultiver l'avoine de Kamschatki etl'avoine de Frise; dans les terrains peu fertiles et stériles, l'avoine de Kildrummic et l'avoine de Shérifque quelques auteurs considèrent, mais mal à propos, comme une variété de l'avoine élancée.

Il est de toute impossibité d'indiquer pour chaque localité les variétés qui lui conviennent, et il n'est pas moins difficile de faire connaitre le rendement de l'avoine; car, plus que toute autre récolte, elle est sujette à de nombreuses variations dans le chiffre de son produit et de ses qualités nutritives. L'hectolitre d'avoine pèse de 34 à 56 kil. 100 kil. d'avoine sont représentés par un équivalent de 180 kil. de foin. M. Royer le fixe, pour la France, à 175 kil. de foin, et d'autres de 116 à $2 \breve{0 ~ k i l . ~}$

L'avoine a fourni, pour 100 parties, 15,7 de gluten el d'albumine, 46,10 d'amidon, 6,7 de matières grasses, 6,0 de sucre, 5,8 de gomme et 21,7 de son.

L'avoine fournit encore à l'économie rurale sa paille; quelques agronomes croient qu'elle est a celle du froment cultivé comme $574: 253$, ou comme $160: 100$. M. Rieffel admet le rapport de $110: 100$.

La paille d'avoine consommée en grande quantité. communique au beurre et au lait une légère amertume : il en est de même des pailles d'orge et de seigle.

L'avoine cultivée constitue un excellent fourrage vert qui est du goût de tous les auimaux; aussi les feuilles et les jets contiennent.ils une forte proportion de matières sucrées qui ont une influence favorable sur la sécrétion laiteuse. On la sème rare- 
ment seule quand elle est destinée à être fauchée, et mieux vaut lui associer quelques plantes légumineuses, telles que vesces, gesses, pois, etc.

Les caractères que l'on doit rechercher dans l'avoine sont un grain bien nourri, pesant, sec; paléoles ou balles minces, membraneuses et tendres; nuances, de celles-ci indistinctes, mais bien prononcées pour leurs variétés; aucune odcur de moisissure ou de champignon.

La paille doit être d'un jaune doré ou d'un blanc jaunâtre luisant, ne présenter aucune couleur grisâtre ou noirâtre, et être privée de toute odeur désagréable : on la donne aux bestiaux sans être coụée.

Avoine orientale, avoine de Hongrie. - La ıariété noire est plus pesante et meilleure que la blanche; l'avoine orientale fournit, dans les terrains riches, un plus grand rapport que l'avoine cultivée, tant en grain qu'en paille; mais ses qualités sont un peu inférieures à celle-ci.

Avorne counte. - Le grain de celle avoine est court et peuriche en matières nutritives, mais plus excitant que l'avoine ordinaire; aussi la culture de cette espèce est-elle très-restreinte en Belgique: on la voit çà et là dans les Ardennes, mais on y attache plus d'importance comme fourragère que comme granifère. A ce titre, clle n'est pas privée de qualité,et il serait à désirer que la culture en prit plus d'extension dans les localités montagneuses et dans les plaines sablonneuses où elle récompense lecultivateur par un fourrage appétissant et abondant. Dans les bonnes terres, il n'est pas rare de la voir prendre une élevation de deux mètres. Nelangée avec des légumineuses, sa culture 

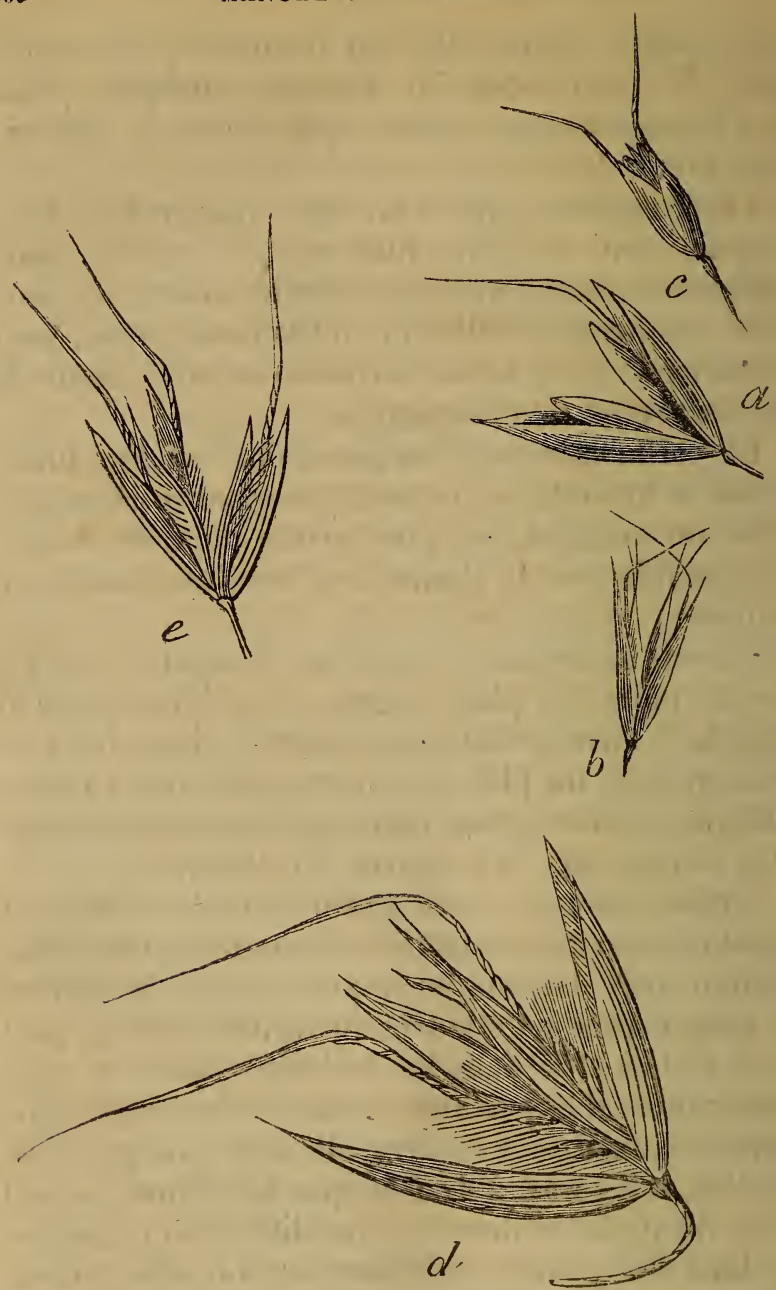

Fig. 89 (1).

(1) $a$ Locuste d'avoine cultivée; $b$ locuste davoine élancéc ; $c$ locuste d'avoine courte; d locuste d'avoine folle; $c$ locuste d'avoine stérile. 
paraitrait encore plus profitable; aucun essai ne nous autorise cependant à rien aflirmer à cet égard.

Avorne Élancée. - Celte plante qui vient dans les terrains pauvres, fournit un grain de médiocre qualité. Elle vaut mieux comme plante fourragère.

Avoine mérissée et avoine folle. - Ces deux espèces, propres aux terrains chauds, se rencontrent çà et là parmi les avoines cultivées. On dit qu'en Ecosse on a obtenu une variété d'avoine folle qui réussit dans les plus mauvaises terres. Cette variété qui nous a été envoyẻe par un agronome distingué de ce pays, nous a paru être la grande avoine blanche cultivée dans quelques arrondissements de la Flandre occidentale; de telle façon que nous ne possédons encore jusqu'ici aucune donnée certaine sur la valeur de cette variété. Si les échantillons que nous avons soumis à une culture soignée sont la vraie avoine folle perfectionnée, il ne peut exister le moindre doute sur la valeur de cette acquisition; mais des essais comparatifs sont nécessaires pour décider la question d'utilité et d'avantage.

Les bestiaux、mangent volontiers ces deux espèces qu'on leur donne quand on les arrache lors du sarclage des champs.

Avonse téxue. - Celte plante qui est annuelle aime les terrains secs, siliceux et calcaires. Elle fournit peu de fourrage qui plait assez aux moutons.

Avolne des prés. - Vivace.

Stations. - Endroits secs, montagneux, fertiles.

Propriétés. - Elle donne un bon fourrage qui dure très-longtemps et que les bestiaux aiment beaucoup.

Rendement. - Cette plante ne donne qu'une coupe abondante de feuilles et des chaumes très- 
nutritifs et un regain qu'on fait pâturer jusqu'aux premières gelées, car sa végćlation est très-tardive.

Elle perd environ les trois quarts de son poids par la dessiccation et fournit 2,100 kilogr. de foin.

Avorne pubescente. - Cette plante qui s'établit de préférence dans les terrains frais, croît aussi très-bien dans les sols secs, sablonneux, les dunes et les terrains calcaires; elle plaît assez aux bestiaux, quoique son foin soit un peu dur. C'est une des plantes les plus productives des prés élevés, car, fauchée ou broutée, elle repousse rapidement. Quand on la sème seule, on emploie 45 à 55 kilogrammes de grains; mais il est préférable de lui associer quelques espèces qui croissent naturellement dans les mêmes conditions : elle donne 6,600 kilogrammes de foin.

Avorne jaunatre. - Cette plante entre dans la composition de toutes les bonnes prairies irriguées.

Propriétés. - Elle est recherchée par tous les bestiaux et à toutes les époques de son développement. Les bœufs et les moutons s'en montrent particulièrement friands tant en vert qu'en sec.

Rendement. - Assez productive pour former le fond des prairies. Elle perd les deux tiers de son poids par la dessiccation : son rendement s'élève a 3,215 kilogrammes de foin.

Avoine précoce et avoine caryophillée. - Ces deux espèces qui plaisent à tous les bestiaux ne donnent qu'un produit insignifiant.

Canche cespiteuse. - Cette plante qui forme des touffes épaisses s'élevant au-dessus du sol, aime les endroits humides et ombragés : les herbivores la recherchent quand ses feuilles sont jeunes; au développement des chaumes, ils n'y touchent plus. C'est donc une espèce propre au pâturage et peu 
TROISIENE PIRTIE. - STITIONS. ETC.

30.3

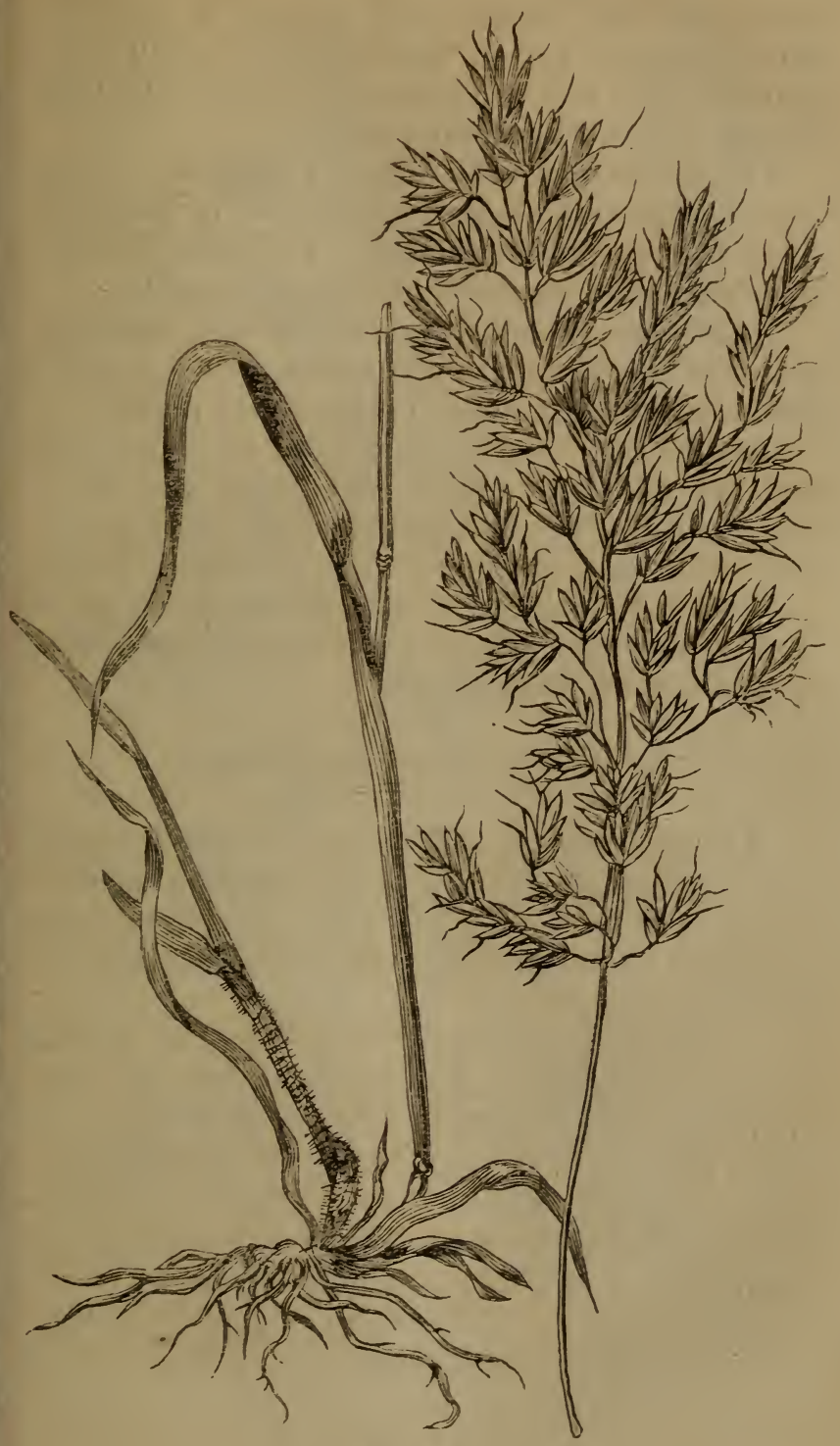

I ig. 91).

Aroine jaunálre. 
convenable au fanage; car quand sa panicule est développée, elle ne donne qu'un foin qu'ils ne mangent qu'à défaut d'autre nourriture. Elle peut donner 3,700 kilogrammes de foin.

Canche discolone et canche flexueuse. - Ces espèces qui sont vivaces se plaisent, la première dans les terrains marécageux, et l'autre dans les terrains secs et montagneux et dans les taillis.

Propriétés. - Elles donnent un bon fourrage, trop court cependant pour être fauché ; mais les boufs et les moutons les paissent avec plaisir.

Rendement. - Elles fournissent un produit peu important à cause de leur disposition en touffes, mais qui n'est pas à dédaigner parce qu'il est obtenu dans des conditions très-médiocres : elles perdent environ les deux tiers de leur poids par la dessiccation. Une pelouse uniforme pourrait donner 3,500 kilogrammes de foin.

\section{ษe tribu. - Anphénathésacées.}

Arrhénathère fausse-avoine. - Cette plante qui présente plusieurs variétés, aime un sol élevé et fertile. Elle redoute les eaux stagnantes, mais prospère, quoi qu'on dise, dans les prés frais et les prairies irriguées.

Propriétés et rendement. - Le bétail en général recherche l'arrhénathère fausse-avoine; les chevaux s'en montrent particulièrement friands, tant verte que sèche: sa végétation est précoce et vigoureuse; car on en obtient jusqu'à trois coupes abondantes. Tout en la faisant entrer dans les mélanges pour la formation de certaines prairies, on peut encore la cultiver seule ou en mélange avec quelques légumineuses. Cette espèce mûrit très-irrégulièrement 
TROISIEAME PARTIE. - STATIONS, ETC.

503

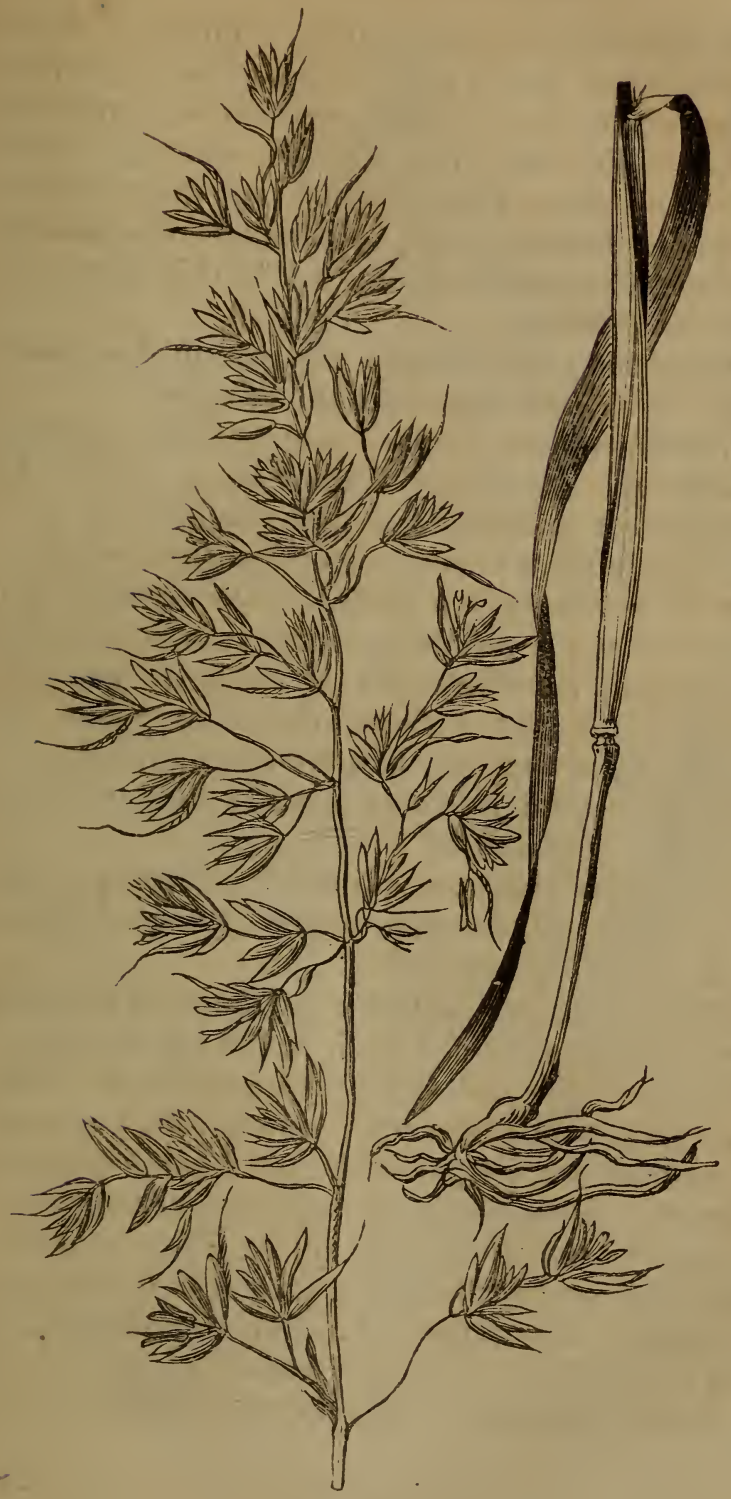

Fig. 91.

Arrhénathère fausze avoine. 
et les fleurons se délachent et tombent à terre. Pour récolter les grains, il faut donc retourner fréquemment sur les lieux, secouer les panicules et recueillir dans des paniers ceux qui sont mûr's : on peut aussi faucher les porte-graines lorsque les sommités de la panicule commencent à jaunir. On emploie de 65 à 75 kilogrammes de grains à l'hectare.

Quand on désire établir une prairie d'arrhénathère, on la sème souvent dans l'avoine.

L'arrhénathère fournit une grande quantité d'herbe que l'on fauche d'assez bonne heure. La floraison est le moment le plus propice. On perd alors sur le poids; mais ce que l'on perd en quantité, on le regagne en qualité. Les éleveurs sont unanimes sur le mérite de celte graminée qui est injustement dépréciée par M. Lecocq, qui s'exprime ainsi : "En résumé, c'est une plante qui produit beaucoup, qui nourrit peu, qui épuise le sol et qui est loin deêtre au premier rang parmi les graminées fourragères. »

La variété bulbeuse, qui attire les mulots et les compagnols, est une mauvaise plante qu'il faut se garder de propagier.

Elle perd environ les trois cinquièmes de son poids par la dessiccation et fournit 6,450 kil. de foin.

Houque nolue. - Cette plante que l'on trouve dans les terrains argileux, glaiseux ou à sous-sol de cette nature, est une herbe que d'après quelques auteurs, les animaux ne mangent qu'avec répugnance et à défaut de toute autre : M. de Gasparin, dont nous partageons l'avis, dit que le bétail en est très-avide. Elle fournit $\mathbf{1 5 , 3 0 0}$ kilogrammes de foin très-riche en principes nutritifs.

Houque laineuse. - On la trouve dans tous les 
terrains sablonneux, argileux et glaiseux, pourvu qu'ils soient substantiels.

Propriétés. - Celte plante assez précoce est aimée par tous les bestiaux, tant en vert qu'en sec. Les moutons la recherchent avec avidité au printemps. Schwerz et De Lingerke l'indiquent comme une mauvaise graminée; mais les expériences de MM. Lequinio, Vilmorin, et de beaucoup d'éleveurs, ne sont pas trés-éloignés de la faire préférer au raygrass et autres analogues.

Rendement. - Elle fournit une grande quantité de feuilles et de chaumes qui repoussent avec facilité, lorsqu'ils ont été fauchés ou broutés. On a conseillé de faire des prairies artificielles composées exclusivement de houque laineuse; mais la disposition naturelle de celte plante à se former en touffes qui se déchaussent au bout de quelques années, doit détourner les cultivateurs de ce conseil : mieux vaut la mélanger avec d'autres espèces.

Elle perd environ les trois cinquièmes de son poids par la dessiccation, et fournit 7,400 kilogrammes de foin.

Hierochloé boréale el hierochloé australe. Ces plantes aiment un sol humide, loameux; elles fournissent un bon pâturage aux troupeaux. Comme la flouve odorante, elles parfument agréablement le foin dans lequel elles se rencontrent. Elles peuvent donner 2,800 kilogrammes de foin et $2,500 \mathrm{ki}-$ logrammes de regain.

Flouve odorante. - Cette plante préfère un sol sec, sablonneux.

Propriétés. - Elle est précoce et peut fournir deux à trois coupes satisfaisantes, car elle repousse promptement; son odeur agréable, qui rappelle celle de la fèvede Tonka, parfume le foin. C'est elle qui 


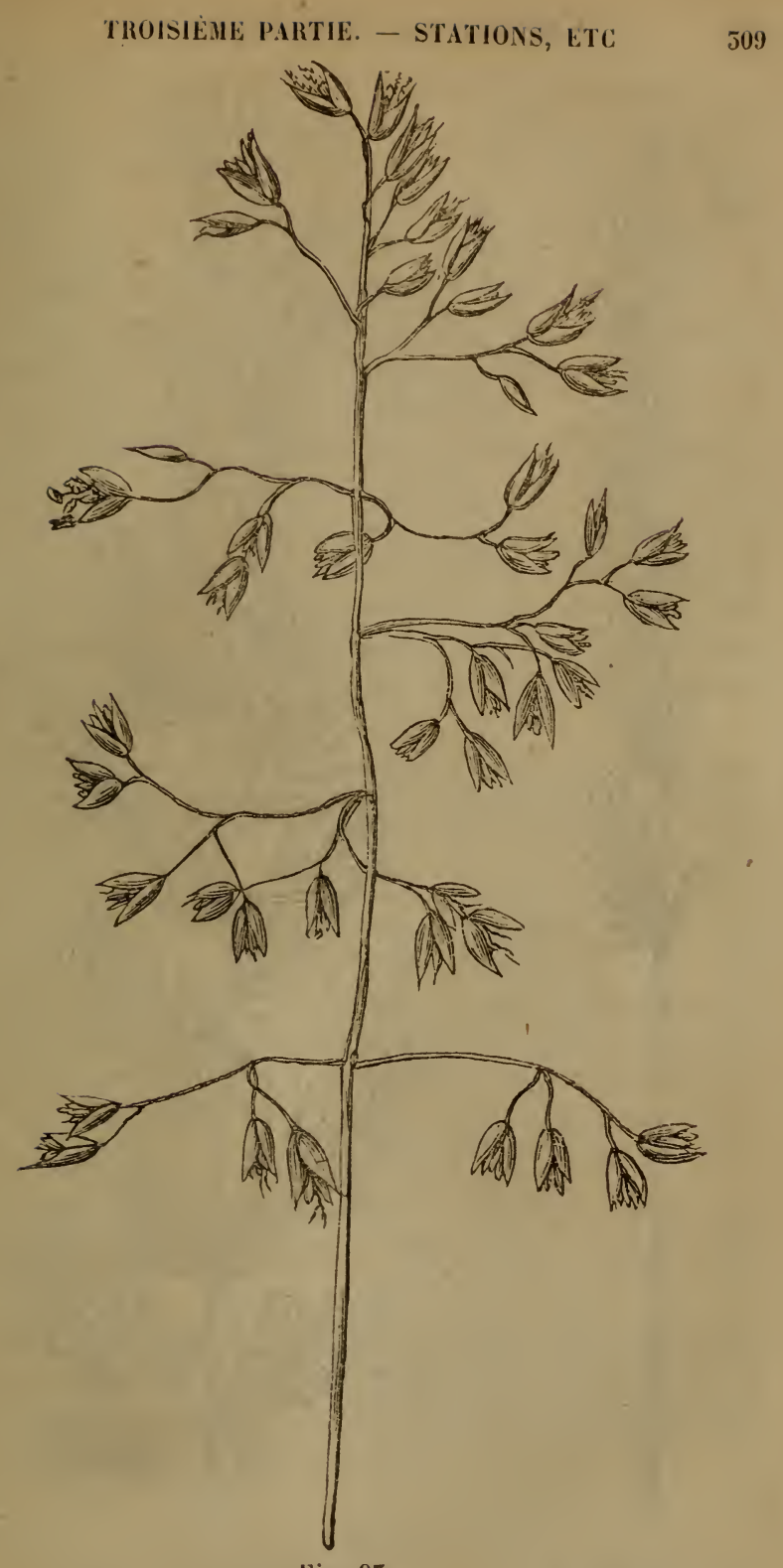

Fig. 95 .

Hierochloć boréale. 


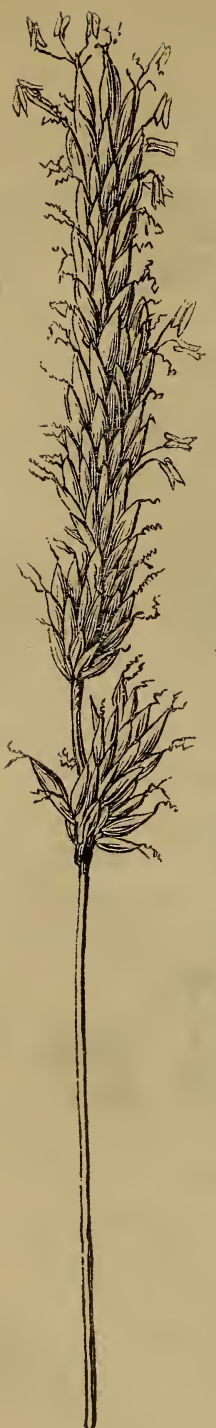

rouve odorante (type).

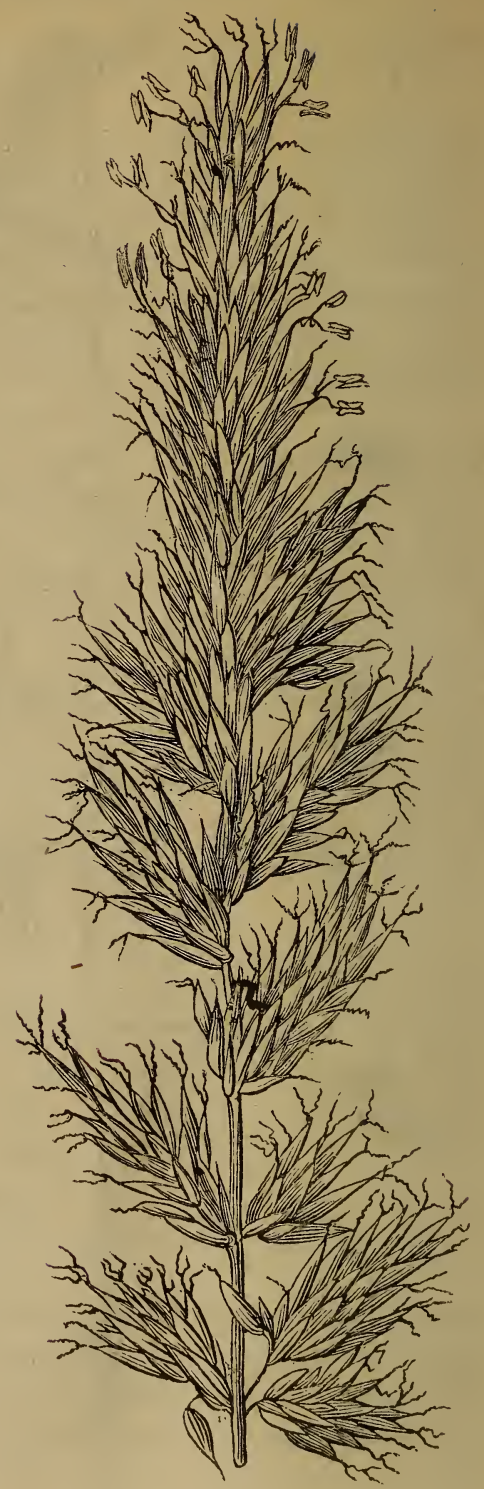

lig. 94. 
caractérise le bon foin, quand il commence à se sécher. Le foin de mauvaise qualité et la paille que les animaux repoussent, deviennent appélissants lorsqu'on y mêle de la flouve. Elle plait à tous les herbivores, tant à l'état d'herbe que de foin, et communique à leur chair une saveur et un parfum particuliers que l'on trouve dans les moutons des Ardennes; mais la flouve est très-commune dans celte localité.

Rendement. - Dans les prairies riches et fertiles, la flouve ne produil pas autant que bien d'autres graminées, mais la qualité supplée à la quantité. Il est des prairies médiocres où la flouve ne réussit pas et qui donnent un bon foin ordinaire; pour améliorer sa qualité et la rendre agréable au bétail, on y mêle un peu de flouve, que pour cela on cullive à part (1).

Elle perd environ les trois quarts de son poids par la dessiccation, et peut donner 2,400 kilogrammes de foin.

\section{vir tribu. - Agyostidicées.}

Calamagrostide des bois, Calimagrostide des Montagnes, Calinagrostide subulée, Caldiagrostide terrestre. - Ces espèces aiment les lerrains secs, ombragés, et ne fournissent qu'un fourrage peu abondant et de qualité très-médiocre.

Calamagrostide lancéolée. - Elle s'établit dans les terrains marécageux ou sur les bords des

(1) Nous avons éfi un jour témoin d’un fail qui mérite d’ètre rapporté : Un éleveur fit l'atequisilion l'une partie de foin composé de bonnes graminées donces. Il le revenlit quelques jours après avee un bénéfiec considérable, après y avoir mèlé un peu de flouve odorante qui y faisaif totalement ciéfaut, et qui fut l'unique cause pour laquelle il se vendit au-dessous de sa valeur réclle. 
étangs et des marais, et fournit un fourrage de qualité inférieure.

Agrostide épi du vent et agrostide interronpue. - Elles se trouvent dans les moissons et plaisent aux bœufs et aux vaches; mais les moutons n'y touchent pas. Elles n'ont aucun intérêt agricole.

Agrostide du Mexique. - Cette espèce qui est vivace et tardive, aime les terrains humides et argileux où elle forme un gazon serré, formé de beaucoup de feuilles succulentes et de chaumes, les uns fertiles, les autres stériles, qui plaisent à tous les herbivores. La fenaison lui enlève environ les deux tiers de son poids

Agrostide vulgaire. - Celte espèce que l'on trouve abondamment sur les bords des chemins, sur les berges des grandes routes et des coteaux, dans les prairies irriguées un peu ombragées et dans les bois, a fourni plusieurs variélés qui contiennent de bonnes herbes fourragères qui plaisent à tous les bestiaux, tant en veri qu'en sec. Elles se modifient selon le sol et les conditions dans lesquels elles se trouvent. Elles perdent un peu plus de la moitié de leur poids par la dessiccation.

Agrostide blancie. - Cette espèce présente des variétés qui ont d'étroites liaisons, quant à leurs propriétés agricoles, avec la précédente; mais une variété dont le mode de végétation est des plus remarquables, connue sous le nom d'agrostide stolonifère, a été l'objet de beaucoup de recherches en Angleterre et ailleurs. Le docteur Richardson la considère comme la meilleure espèce des graminées agricoles. En France et en Belgique, le fameux fiorin grass des Anglais est loin de jouir de la mème faveur. Cependant, l'agrostide stolonifère peut être utile dans les prairies basses et presque 
TROISIÈie PARTIE. - STATIONS, ETC.

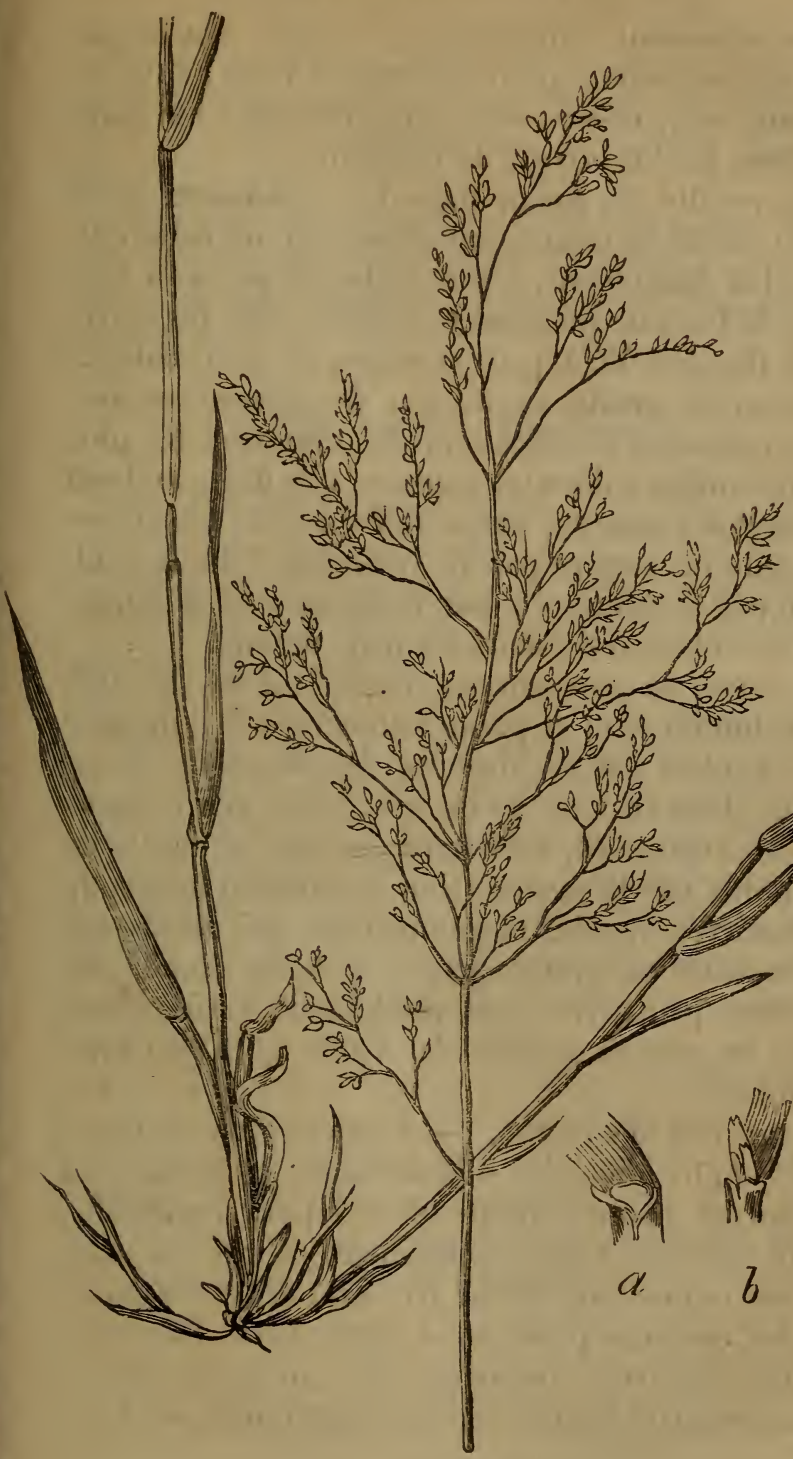

Fig. 93 (1).

Agrostide vulgaire.

(1) $a$ Portion cle feuille appartenant à l'agrostide vulgaire ; $b$ portion de feuille de l'agrostide blanche. 
continuellement submergées, où elle fournit un fourragesavoureux pour les bêtes à laine; dans les lerrains sees, elle devient dure et sèche au point que tous les herbivores la refusent.

La récolte du fiorin dans ces conditions n'est pas la chose la moins embarrassante de celle culure. En Angleterre, on ne se borne pas à la faucher, la faux ne pouvant retrancher que les extrémilés des jets déjà peu allongés de leur nature, mais on la gratle au moyen de râleaux en fer. Celle agrostide constitue un des fourrages les plus lardil's : mêlés à d'autres espèces, elle finit, au bout de quelques années, par élouffer toules les autres dont elle ne souffie pas le voisinage. On la multiplic par le semis ou par firgments de chaumes pourvus chacun de deux ou mois nocuds.

L'agrostide stolonfène récoltée dans les terrains humides et à peine submergés est mangée avec avidité par les chevaux, les moutons el les boufs, tant en vert qu'en sec; celle des terrains sees est repousséc. Elle perd environ la moilié de son poids par la dessiccalion et contient beaucoup de matières sucrées el gommeuses qui paraissent s'accumuler en grande partic dans les nocuds des chaumes primaires. Son rendement varie beaucoup; on peut en obtenir de 4,000 à 9,000 kilogrammes.

Agrostine des cumens. - Celte espèce se développe lantôt dans les sols humides, fangeux, où elle forme une excellente pature pour les boeul's; lantôt dans les lieux monlagneux, élevés el sees, où elle donne une herbe très-finc el savoureuse, que les moutons préfèrent à loule autre. Elle perd environ les trois cinquiemes de son poids par la dessiccation el fournit $5, ! 00$ kilogrammes de foin. 
Luguriar ovale. - Celle plante forme des toufles de fenilles qui ne plaisent guère aux bestiaux; élant anmuelle, elle n’a ancune utilité agricole marquéc.

Amophute nes sabues. - Celle espèce qui a pour destination spéciale de fixer l'arène el le sable maritime, fournit un fourrage peu recherchí par le bélail.

vIc tribu. - sripacées.

Stipe mapenne el stipe curvelu. - Ces plantes qui se développent en touffes, cà et là, sur les collines sèches. sablonneuses et calcaires, fournissent uII fourrage peu abondant, que les bestiaux refusent souvent de manger.

Le hasiagrostide Calanagrostide qui est très-rare en Belgique, dans les bois montagneux à sol un peu compacte, humide, donnent des touffes de feuilles el des liges dures et si peu succulentes que les moutons et les chèvres ne les mangent pas toujours, même au printemps, quoiqu'elles soient trèsprécoces. Les boufs le mangent volontiers, quoique son foin soit peu nutritif. Il ne perd que la moitic de son poids par la dessiccation.

Piptatiène multiflone. - Celle plante qui aime les lieux ombragés, prospère dans les laillis, où elle fournit un fourrage précoce, délicat et assez abondant, qui plaî̀ à tous les herbivores. Elle peut fournir deux ou trois coupes, qui perdent les deux liers de leur poids par la fenaison. 


\section{VIII tribu. - Miliacées.}

Milier epars. - Cette espèce qui est assez commune dans les bois sous la haute futaie, prospère dans les terrains riches en humus; elle plait dans sa jeunesse au bétail qui la dédaigne après. Elle perd un peu plus des deux tiers de son poids par la dessiccation.

Le foin de milier épars exhale une odeur agréable.

\section{IX tribu. - Cyrrodonées.}

Chiendent dactrle. - Il se plait dans les sols sablonneux, qu'il envahit souvent à l'exclusion de toute autre plante : son fourrage, tout en étant goûté par le bétail, ne peut compenser les torts qu'il fait à la culture des champs qui peuvent produire des espèces plus productives et de meilleure qualité ; ses rhizomes longuement traçants, mêlés avec l'avoine, peuvent servir à la nourriture des chevaux.

\section{$X^{e}$ tribu.- Hohalavpidacées.}

Asprelle faux-riz. - Celte plante qui aime de préférence les bords des eaux courantes, se plaît aussi dans les terrains marécageux, où elle prend un grand développement et fournit beaucoup de fourrage qui est recherché par tous les bestiaux, malgré les aspérités dont ses feuilles et ses chaumes sont ordinairement couverts.

Mibore naine. - C'est la plus petite des graminées annuelles indigènes; elle s'établit dans les sols arides, sablonneux et crayeux, où elle forme 
de petites touffes denses, que les moutons broutent en mars.

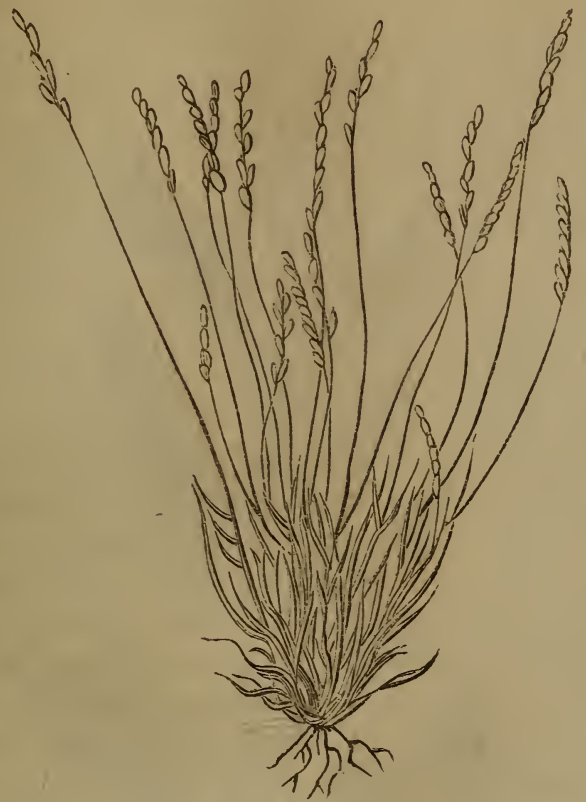

Fig. 96.

Mibore naine.

Alpiste roseau. - Cetle espèce qui est commune sur les. bords des ruisseaux, des rivières et dans les endroits marécageux, mérite d'attirer l'attention de l'agriculteur. Dans les glaises sablonneuses, humides, elle donne un produit considérable et de très-bon fourrage que les bœufs et les raches recherchent surtout.

Elle peut donner trois coupes; mais on doit la faucher lorsque la panicule est sur le point de sor- 


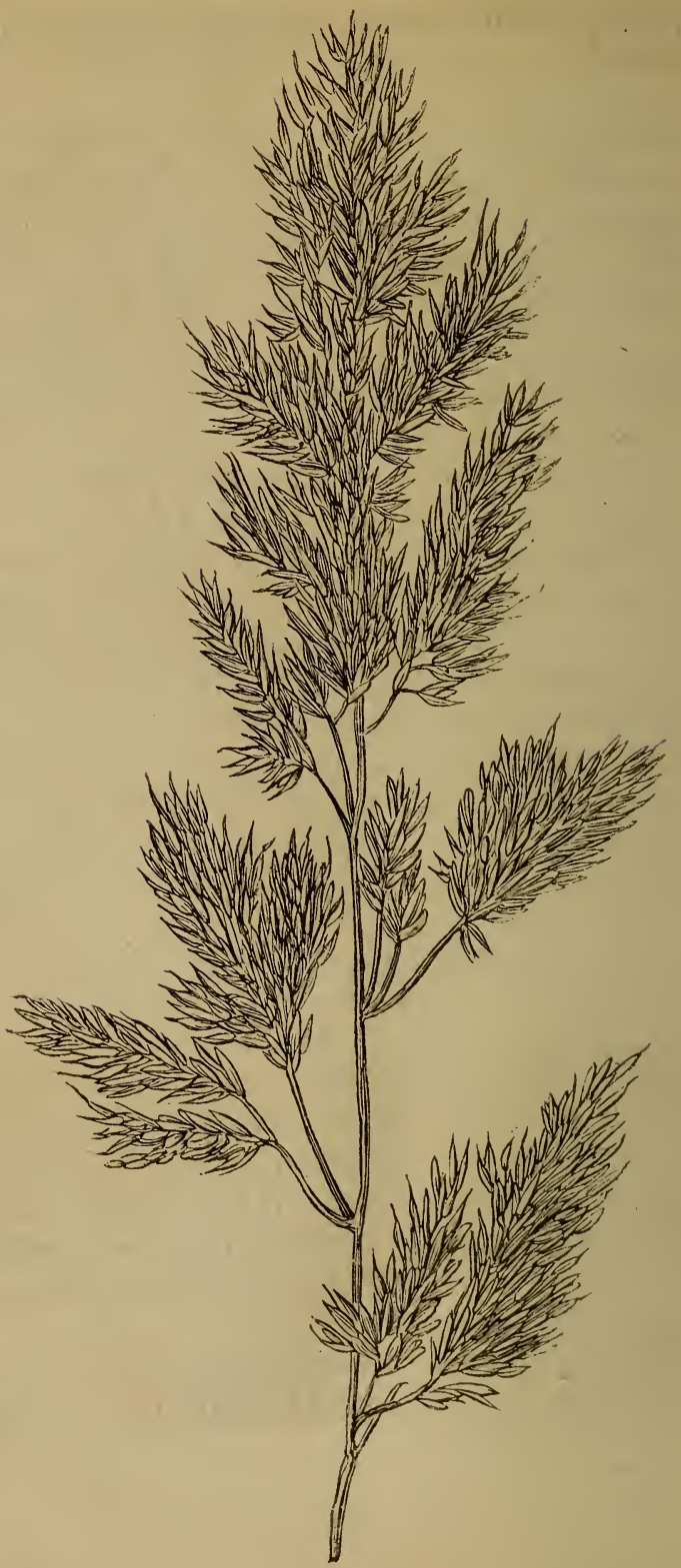

lig. 97.

Alpisle rosean. 
tir : la fenaison lui fait perdre un peu plus de la moitié de son poids; son produit en foin sèlève à 15,800 kilogrammes.

Cette plante, dont une variété à feuilles panachées se cultive dans les jardins les moins fi'ais où elle réussit très-bien, peut aussi être d'une certaine utilité dans les terrains escarpés, secs, calcaires, où elle produit moins de fourrage, mais que les vaches consomment très-bien.

Alpiste des Canaries, alpiste aquatique el alpiste RONGÉE. - Ces trois espèces sont cultivées, tantôt comme céréales, tantôt comme fourragères : comme céréale, l'alpiste des Canaries convient dans les terres très-fertiles plutôt sèches qu'humides; l'alpiste aquatique produit plus dans les terres fraîches. Le rendement de l'alpiste rongée est toujours le plus faible.

Comme fourragère, l'alpiste rongée est celle qui emportera la préférence, parce qu'elle donne le plus grand produit sans distinction de sol. Tous les animaux aiment cette fóurragère, tant en sec qu'en vert; sa paille est préférée à celle des autres céréales. Elle perd un peu plus de la moitié de son poids par la dessiccation.

Fléole des sibles et fléole rude. - Ces deux espèces, qui aiment les sols sees, ne sont pas recherchées par les bestiaux; les moutons les broutent à peine dans leur jeunesse.

Fléole uérissée. Fléole lisse. - Ces deux espèces, que l'on rencontre dans les bois montagneux ct sur les collines sèches, fournissent dans leur jeunesse une pâture très-substantielle et agréable aux moutons et aux chèvres qui les recherchent avant qu'elles se durcissent.

Fléole des prés. - Cẹtle plante, que les An- 
MANUEL DES GRAMINÉES.

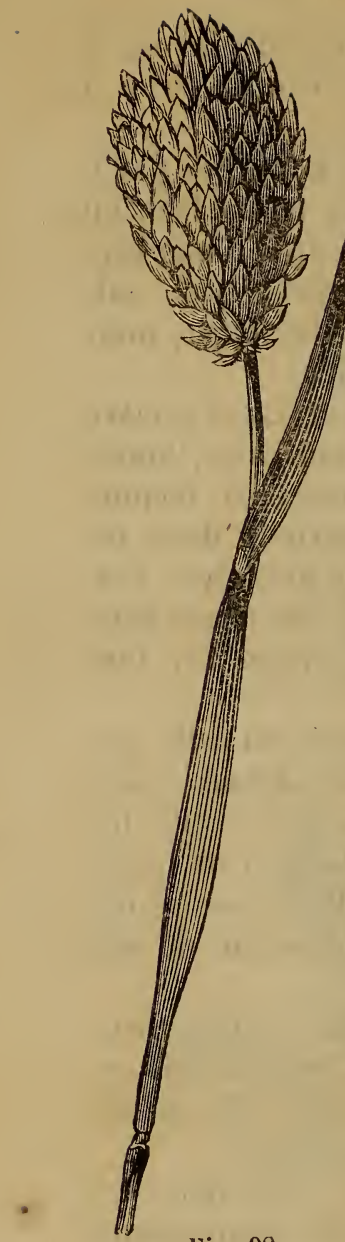

glais nomment thimoy-grass, aime les terrains bas, humides et presque marécageux.

Propriétês. - On a apprécié diversement cette plante: les uns lai reconnaissent des qualités médiocres, à cause de sa tardivité ; les autres l'estiment beaucoup et l'envisagent comme une des meilleures graminées, à tel point que c'est par la présence de cette plante qu'ils jugent de la valeur d'une prairie. Sans partager les exagérations des uns et des autres, nous regardons la fléole comme une graminée qui plaît à tous les bestiaux, mais notamment aux chevaux; qui fleurit, à la vérité, tard, mais qui ne laisse pas de produire de très-bonne heure beaucoup de fascicules de feuilles. Les lièvres et les lapins aiment beaucoup la fléole.

Rendement. - Le thimothée donne beaucoup de fourrage vert et sec : semé seul, il peut produire, dans des circonstances favorables, de 7 à 18,000 kilogrammes de foin par hectare; mais ce rende-

rig. 98. ment est exceptionnel et ne peut Alpiste des Canaries. être pris pour la règle. En Belgique, on ne le sème qu'en mélange. Il donne une première coupe abondante et un regain tardif, el perd environ les cinq huitièmes de son poids par la dessiccation. Le choix des semences provenant de 
TROISIĖIE PARTIE. - STATIONS, ETC.

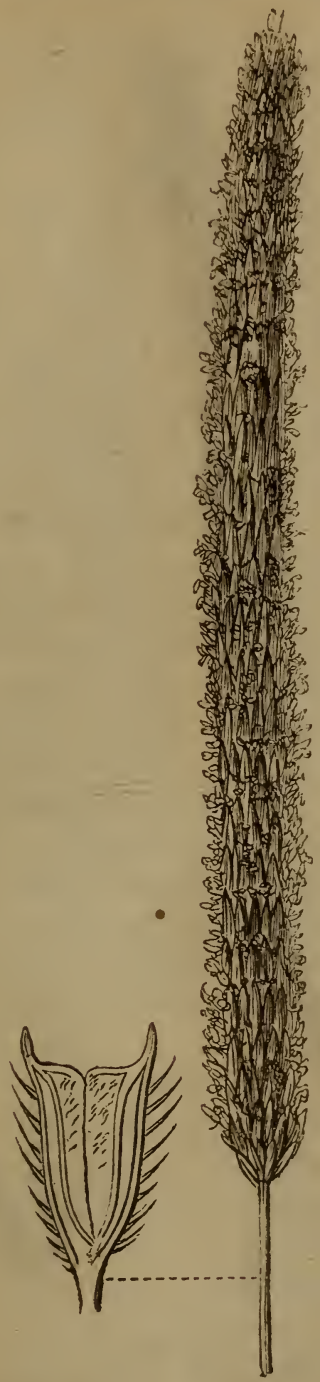

Fig 99.

Fléole des prés.

27. 


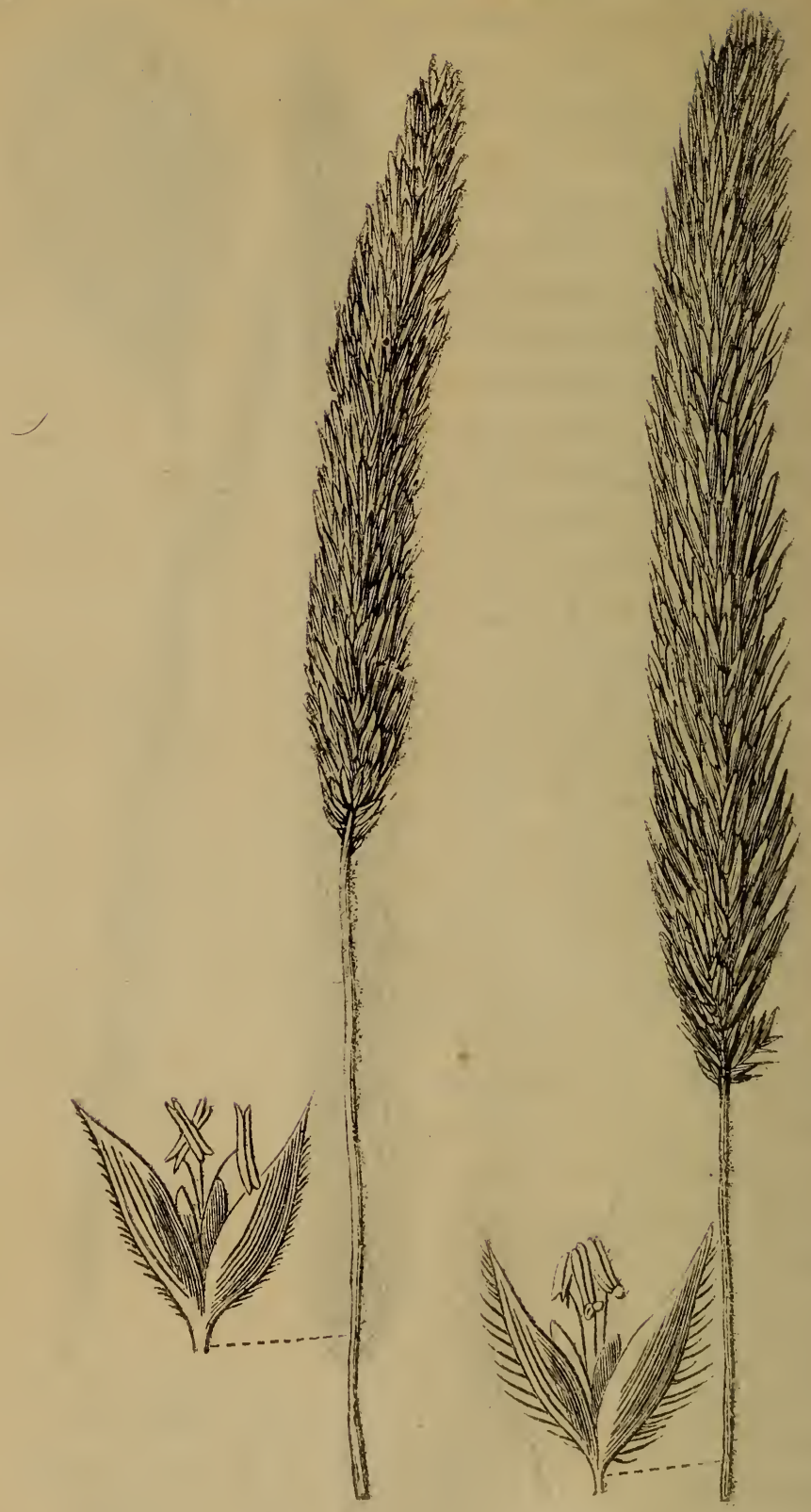

fig. 100.

Fliole lisse.

Fléolc hérisséc 
TROISIĖUE PARTIE. - STATIONS, ETC.

pays étrangers, et de préférence la semence anglaise, contribue pour beaucoup au rendement. On emploie 8 à 9 kilogrammes de grain à l'heclare.

La fléole noueuse, qui n'en est qu'une variélé, est pâturée avec plaisir par les moutons el les boufs, et aime une glaise argileuse.

Fléole des Alpes. C Celle espèce, qui est infiniment plus petite que la précédente, se trouve dans les lieux montagneux, sablonneux el les dunes, el fournit une pâture savoureuse et substantielle.

VulPIN des PRÉs. - Il aime de préférenceà tout autre, où il ne réussit pas bien, un sol humide ou frais, quelle que soit d'ailleurs la nature du terrain. Il s'élablit à proximité des rigoles ou des fossés qui sillonnent les prairies.

Propriétés. - Celle plante qui entre dans la composition des prairies temporaires et permanentes, fournit un fourrage à la fois précoce et abondant que tous les bestiaux aiment à l'envi.

Rendement. - Elle atteint une grande taille, produit beaucoup de fascicules de fenilles à la base, pousse de très-bonne heure des feuilles larges, molles et savoureuses, el ses chaumes contiennent beaucoup de matières sucrées; elle fleuril en mai: Le vulpin des prés peut donner trois

Fig. 101.

Vulpin des prés. 

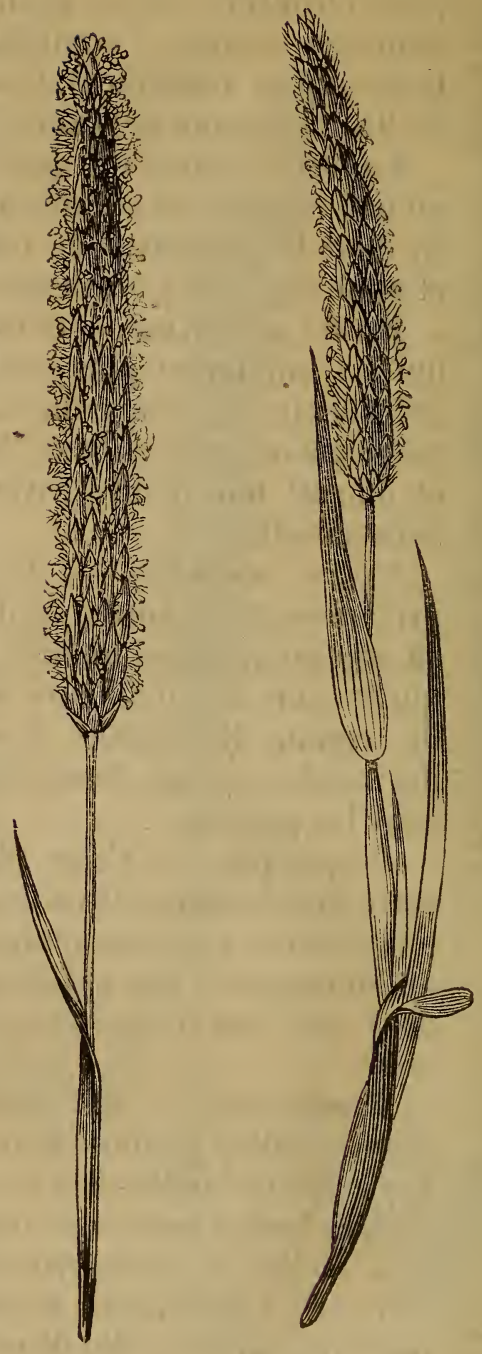

Fig. 102.

Vulpin genouille. Vulpin fauve. 
coupes, si la première est faite avant la floraison, et un regain des plus abondants. La fenaison lui enlève environ les deux tiers de son poids; elle fournit 6 à 7,000 kilogrammes de foin dans les terrains humides, et 2,400 kilogrammes de foin, $y$ compris le regain, dans les solsfrais. Le foin a une odeur agréable. On emploie 19 à 23 kilogrammes de grains à l'hectare quand on le sème seul.

Vulpin des chanps et vulpin utriculé. - Ces deux espèces que l'on trouve, la première, dans les lieux cultivés et l'autre dans les prés, forment de très-bon fourrage que l'on donne au bétail lors. des sarclages.

VulpiN bulbeux, vUlpin GeNoullé et vulpiN Fauve. - Le vulpin bulbeux ne s'établit que dans les prés salés et sur les bords de la mer. Les autres se plaisent dans les marais tourbeux, les fossés vaseux et les mares.

Propriétés. - Elles fournissent un fourrage que tous les herbivores mangent avec plaisir, et il n'est pas rare, comme le dit très-bien Lecoq, de les voir s'exposer quelquefois à s'enfoncer dans la vase pour aller les chercher.

Rendement. - Elles fournissent un fourrage assez abondant qui devrait engager tous les agriculteurs à les multiplier dans tous les prés assez humides qui permettent leur développement : ils perdent les deux tiers de leur poids par la dessiccation.

Polypogon de Montpellier et des rivages. Ils fournissent un fourrage insignifiant. 
Xre tribu. - Paivicucées.

Tragier en grappes et gastridie lendigère. - Ces plantes, qui fournissent très-peu, ne plaisent guère aux bestiaux.

Sétaire verticillér, SÉtaire gladque el SÉtaire verte. - Ces espèces, qui pullulent souvent dans les guérets et qui n’ineommodent pas moins les maraichers, peuvent se donner aux herbivores qui les aiment beaucoup. Quoique la première donne un fourrage assez abondant, il serait imprudent de la semer à cet effet.

Sétaine d'Italie. - Cette espèce est cultivée comme plante granifère et fourragère. La variété dite de Germanie réunit le mieux les deux conditions, quand on la cultive dans un sol plutôt sec qu'humide; mais on lui préfère quelquefois la variété à longues soies, dans l'intention d'en éloigner les petits oiseaux, quir sont très-friands de ses grains.

Digitaire sanguine, digitaire glabre el oplisuène PIED DE COQ. - Ces espèces sont recherchées par tous les bestiaux, mais elles ne peuvent être cultivées avec avantage, comme l'a dit à tort M. Bose.

Panis millet, millet des oiseaux. - Celte plante est cultivée comme céréale el lemande, pour réussir, un terrain un peu humide, meuble, chaud ef fertile. Les variétés qui ont été établies d'après la couleur des paléoles n'ont aucune importance agricole ou économique.

Un hectare produit de 50 à 57 hectolitres de grains; l'hectolitre pèse de 69 à 72 kilogrammes.

Un hectolitre de grains cortiqués, pesant 70 kilogrammes, en donne 43 kilogrammes quand ils sont décortiqués. 


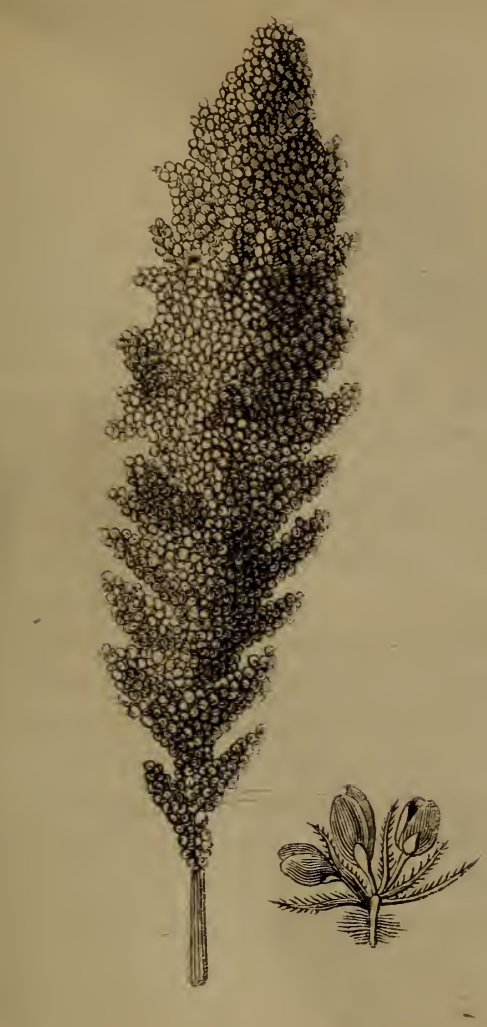

Fig. 103.

Setaire de Germanic.

Un hectare fournit de 4,500 à 6,800 kilogrammes de paille qui est l'une des meilleures pour la nourriture du bélail ; 150 kilogrammes de paille de millet éfuivalent à 100 kilogrammes de foin ordinaire. 


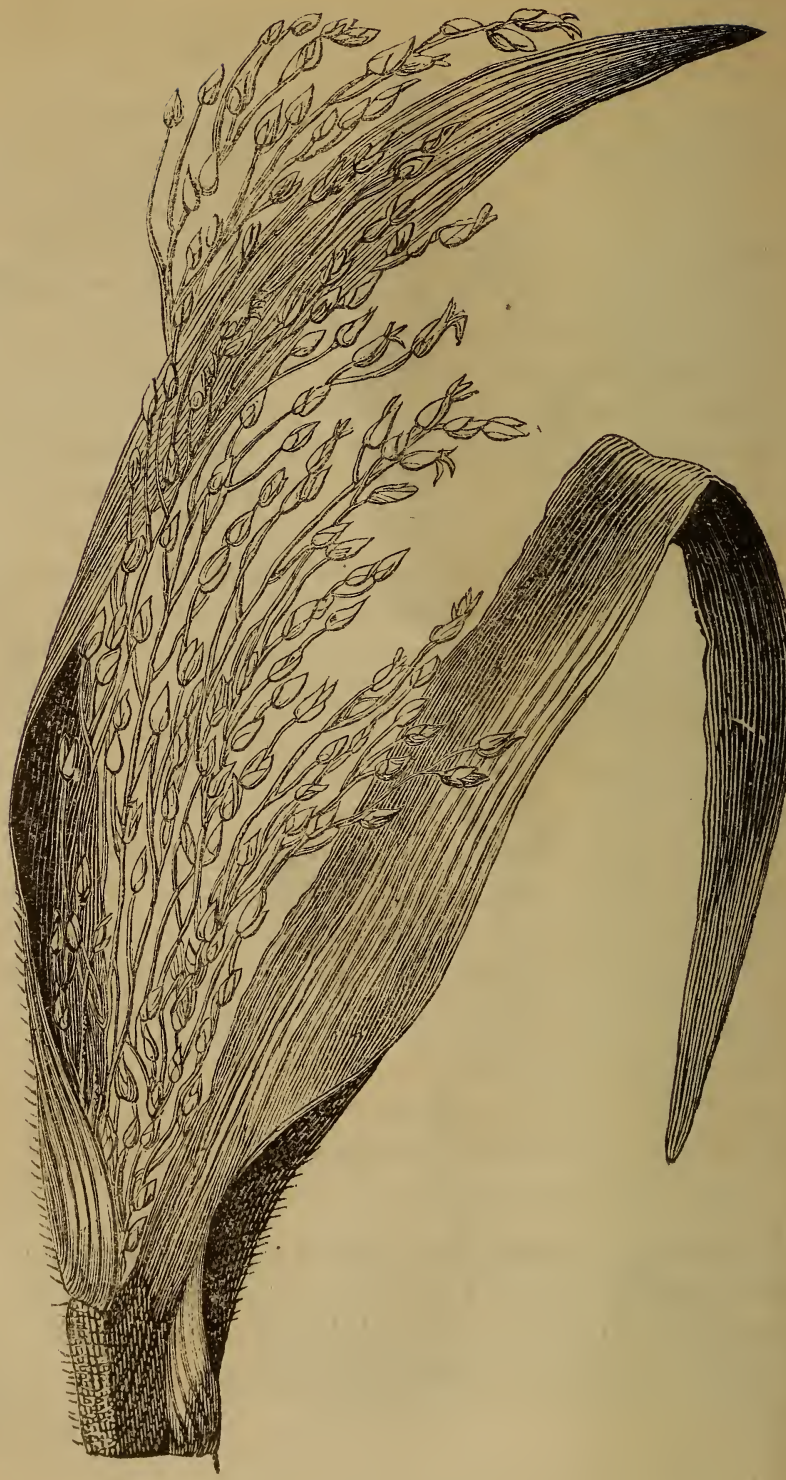

Fig. 104.

panis millet. 
Panis capillaire.-Plait aux bestiaux, mais n'a aucun intérêt agricole.

Panis élevé. - Cette plante a été en Belgique l'objet de quelques essais; elle a fourni une énorme quantité de fourrage que tous les bestiaux mangent avec avidité; mais, comme elle réclame un sol fertile, chaud et meuble, et qu'elle ne convient pas dans les terres médiocres où elle ne donne qu'un produit peu satisfaisant, nous croyons que sa culture ne se propagera pas.

Sorghier vulgaire. - La culture de cette espèce de houque de Linné a été vivement recommandée en Belgique; mais tous ceux qui l'ont entreprise dans le but d'obtenir des grains, ont été déçus dans leur espoir. Aussi cette plante a-t-elle eu le sort du madi cultivé.

On a aussi prôné beaucoup la culture du sorghier saccharin; mais jusqu'ici on ne possède aucune donnée positive sur la valeur de cette espèce.

Barbon digité. - Cette plante, qui ne s'établit que dans les terrains sablonneux, dans les dunes et les sables maritimes, n'a aucune qualité agricole, car les moutons et les chèvres même ne touchent pas à ses feuilles, quand ils trouvent quelque autrc herbe. 


\section{GRAMINÉES FOURRAGÈRES}

I.ES

pins convenables pour les alifférents sols et stations (1).

Avec les données que nous avons acquises sur les stations, les propriétés et le rendement des gra-

(1) Comme beaucoup d'agronomes croient avec plus ou moins de raison que l'eau fait les graminées, nous mettons sous les yeux du lecteur la classification adoptée par HI. de Gasparin; mais, à notre avis, les nombreuses espèces comprises dans chaque groupe rendent le choix pour la formation des mélanges assez difficile : c'est pourquoi nous avons jugé utile de passer en revue les divers sols et stations et d'indiquer les plantes qui y croissent avec le plus de vigueur.

Première division. - Plantes des terrains humides.

Toutes ces plantes ne prospèrent que quand leur's racines sont en contact avec l'cau.

Fétuque des prés.

Enorlic bleue.

Glycérie aquatique.

Glycérie flottante.

Catabrose aquatique.

Roseau à balais.

Agrostide blanche.

Alpiste rosean.
Fléole des prés.
Vulpin des prés.

On y trouve les légumineuses suivantes :

Gesse des prés.

Vesce des haies.

Lotier des marais. 
minées indigènes ou exotiques qui peuvent ètre cultivées avec avantage, nous pouvons maintenant donner quelques conseils sur le choix des espèces propres à établir des prairies artificielles temporaires et des prairies naturelles permanentes.

a division. - Plantes des terrains frais.

Orge des prés.

Ivraie vivace.

Cynosure crételle.

Fétuque fausse ivraie.

Fétuque des prés.

Brome dressé.

Glycérie maritine.

Glyeérie distante.

Dactyle glomeré.

Paturin des bois.

Paturin commun.

Paturin des prés.

Avoine pubeseente.

Canche cespiteuse.

Arrhénathère fausse aroine.

Houque laineuse.
Hierochloë boréale.

Floure odorante. Agrostide vulgaire. A yrostide des chiens. Fléole des prés.

Vulpin des prés.

On y trouve les légumineuses suivantes :

Trèfle des prés.

Vesce des haies.

Vesce à bouquet.

Gesse des prés.

Luzerne cultivée.

Luzerne houblomnée.

$\mathbf{3}^{\mathrm{e}}$ division. - Plantes des terrains secs.

La propriété de ces plantes est de résister à la sécheresse; mais leur produit dépend des intervalles de fraicheur du sol pendant lesquels seulement elles peuvent pousser.

Elyme des sables.

Froment traẹant.

Ivraie vivace.

Nard élancé.

Cynosure crételle.

Fétuque queue de souris.

Fétuque polymorphe.

Fétuque glauque.

Fétuque rouge.

Fétuque durette.

Brome inerme.

Brome rude.

Brome seiglin.

Brize moyenne.

Paturin eommun.

Paturin comprimé.

Koelérie crètée.

Avoine des prés.

Avoine jaunàtre.

Canche flexueuse.

Houque molle.
IIouque laineuse.

Hierochloë boréale.

Agrostide vulgaire.

Stipe empenné.

Chiendent dactyle.

Vulpin des champs.

Digitaire sanguine.

On y trouve aussi les plantes suivantes :

Achillée mille-feuilles,

Lotier corniculé.

Lotier maritime.

Orobe tubéreux.

Trèfle rampant.

Trèfle couché.

Trèłle fragifère.

Coronille variée.

Serradelle naine.

Sainfoin. 
I. - Prairies temporaires.

GRAMINÉES ANNUELLES.

$1^{0}$ Terres de bruyère, humides, maigres:

Ivraie rieffel.

$2^{\circ}$ Sables argileux, rudes et caillouteux, secs en été, très-humides en hiver:

Ivraie rieffel. Var. sub-mutique.

La première coupe de ces espèces se fane ou se donne en vert; le regain peu abondant est pâturé.

$\overline{5}^{\circ}$ Terrains secs, pauvres, montagneux :

Avoine courte.

$4^{\circ}$ Terrains secs peu fertiles :

Alpiste rongée.

$\breve{\zeta}^{\bullet}$ Terrains fertiles, argilo-sablonneux, sablonneux ou marneux :

Orge vulgaire d'hiver.

6 Terrains sablonneux ou loameux :

Seigle multicaule.

Ces deux espèces ne fournissent qu'une coupe, après laquelle le sol produit encore, la même année, d'autres récolies. 
$7 \circ$ Terrains loameux chauds :

Maïs cultivé.

N.B. Les espèces précédentes se mêlent souvent avec quelques autres plantes fourragères légumineuses, sauf le maïs qui se cultive presque toujours seul.

\II. - Prairies temporaires durant $2 \dot{a} \zeta$ ans.

On peut semer isolément chacune des graminées suivantes, selon les indications données à chaque article spécial :

a. Orge bulbeuse.

$b$. Ivraie d'Ttalie.

c. " vivace.

d. Glycérie aquatique.

e. Arrhénathère fausse avoine.

$f$. Flouve odorante.

g. Fléole des prés.

$h$. Vulpin des prés.

\section{S III. - Prairies permanentes.}

$1^{0}$ Sols secs, sablonneux, non irrigables:

Seslérie bleue.

Fétuque des brebis.

Canche flexueuse.

Brize moyenne.

Avoine des prés.

Brome dressé.

Flouve odorante. Agrostide vulgaire.

On associe à ces graminées un peu de pimprenelle, de lupuline et de lotier corniculé (à pa(urer); ou bien :

Seslérie bleue.

Féluque des brebis. 
Fétuque rouge.

" durette.

Canche flexueuse.

Fléole des Alpes.

Agrostide vulgaire.

Cynosure crételle.

Paturin des Alpes.

Avoine pubescente.

On leur associe ordinairement du lotier corniculé, du trèfle rampant, du trèfle couché, de l'anthyllide vulnéraire, de la vesce des haies. (A pâturer.)

$2^{\circ}$ Sols sablonneux frais et ombragés, non irrigables :

Paturin des bois.

" prés.

Avoine jaunàtre.

Agrostide vulgaire.

Dactyle pedotonné.

Mélique penchée.

On leur associe souvent du trèfle rampant et du lotier corniculé. (A pâturer.)

$3^{\circ}$ Terrains sablonneux, frais et inondés quelquefois par les eaux de la mer :

Fétuque durette.

" rouge, var. maritime.

Vulpin bulbeux.

Aelbroeckie maritime.

Glycérie maritime.

(c) distante.

Sclérochloë couché.

Koelérie crêtée, var. de l'arène.

Paturin des prés.

Agrostide blanche, var. maritime.

On y ajoute du lolier maritime, du trèfle des prés et du plantain maritime. 
$4^{\circ}$ Terrains sablonneux, susceptibles d'irrigation (eau douce) :
Agrostide vulgaire.
Paturin commun. " des prés.
Avoine jaunâtre.
Fétuque des prés. Houque laineuse.
Avoine pubescente.

On y mêle du lotier corniculé, du trèfle rampant, du trèfle couché et de la gesse des prés.

$5^{\circ}$ Terrains calcaires secs, non irrigables :
Ivraie vivace
Brome dressé.
Dactyle pelotonné.
Koelérie en crête.
Seslérie bleue.

On y ajoute du trèfle couché, du boucage saxifrage, de la coronille variée, du trèfle blanc et du sainfoin.

$6^{\circ}$ Terrains calcaires irrigables :

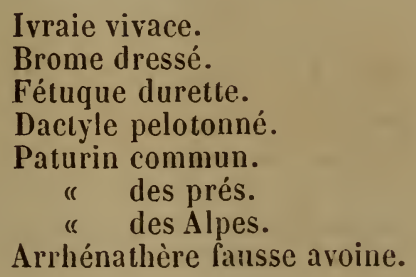

On y mêle du sainfoin, du trèfle des prés et fragifère.

$7^{\circ}$ Terrains argileux secs : 
Ivraie rieffel, mutique.

Dactyle glomeré.

Paturin des prés.

Fétuque des prés.

Paturin des bois.

Alpiste roseau.

Paturin des Alpes.

On y ajoute de la gesse des prés et du lotier corniculé.

$8^{\circ}$ Terrains argileux susceptibles d'irrigation:

Vulpin des prés,

Paturin commun.

" des prés.

Fléole des prés.

Fétuque des prés.

" fausse ivraie.

Avoine jaunàtre.

Ivraie vivace.

On leur associe de la gesse des prés et des vesces de haie.

9० Terrains argilo-sablonneux ou argilo-calcaires susceptibles d'irrigation.

(Composition propre au pâturảge):

Flouve odorante.

Fléole des prés.

Cynosure crételle.

Avoine jaunâtre.

Fétuque fausse ivraie

Ivraie vivace.

Orge des prés.

Paturin commun.

“ annuel.

On leur associe ordinairement des vesces à bouquet, du trèfle rouge, du trèfle blanc et du trèfle couché. 
(Composition propre au fauchage):

Ivraie vivace.

Arrhénathère élevée.

Paturin des prés.

" commun.

" annuel.

Agrostide vulgaire.

Vulpin des prés.

Féluque des prés.

“ fausse ivraie.

Cynosure crételle.

Dactyle pelotonné.

Flouve odorante.

On y mêle du trèfleblanc et rouge, de la minette, du lotier corniculé et des vesces à bouquet.

$10^{\circ}$ Terrains marécageux ou marais desséchés.

\section{Sol très-humide :}

Glycérie flottante.

Fléole des prés.

Catabrose aquatique.

Alpiste roseau.

Fétuque roseau.

" fausse ivraie.

Vulpin des prés.

“ genouillé.

On y ajoute de la gesse des prés et des marais, de la vesce des haies et du lotier des marais.

Sol tourbeux :

Fléole des prés.

Vulpin géniculé. 
Vulpin fauve.

Agrostide des chiens.

(" blanche.

On leur associe des vesces à bouquet et des haies, et du lotier des marais.

\section{Sol inondé :}

La glycérie aquatique, la glycérie flottante et l'alpiste roseau sont les seules espèces qui réussissent dans cette condition : on leur associe quelquefois du seneçon, des épilobes et des salicaires; mais ces plantes ne sont guère utiles, notamment les dernières. 


\section{TABLE SYNONYMIQUE}

\section{DES ESPÈCES.}

AelbroeckIE MARITIME N. Aelbroeckia maritima $N$. Poa littoralis Gouan. Dactylis litloralis Wldw. D. maritima Schrad. D. Distichophylla Brignol. Poa pungens Bieb. Agrostis pungens Pall. Poa tunetana Spreng. Poa repens Bicb. Triticum supinum Schrank. Calotheca littoralis Spreng. Nom vulgaire. Paturin des rivages.

AGRoStIDE blanche. Agrostis alba Will. A. alba Schrad. A. stolonifera $L$. A. capillaris Poll. A. gigantea $R$. A. maritima $V$. Hall. A. hybrida Dmrt. A. diffusa Host. A. tenella Hoffm. A. decumbens Gaud. A. densa Bieb. A. byroïdes Dmrt. v. v. Eternue drageonnée. Foin rampant. Trenime. Trainasse. Foin blanc. Fiorin. Agrostide blanche. A. rouge. A. pâle. A. géant. Foin capillaire.

ACROSTIDE DES CHIENS. Agrostis canina $L$. Trichodium caninum Schrad. Agraulus caninus $P . d$. B. Agrostis varians Thuill. A. vinealis Schleich. A. pallida Schkuhr. A. alpina Leyss. A. hybrida Gaud. Trichodium hybridum $R$. et $S$. Tr. diffusum Link. Agrostis pusilla $D m t r$. A. vivipara $L e j$. A. aurea $L e j$.

AGROSTIDE ÉPI DU VENT. Agrostis spua venti $L$. Anemagrostis spua venti Trin. Agrostis purpurea Gand. Apera spica venti $P$. $d . B$. Apera purpurea Ruch. N. v. Jouet du vent. Panache des champs. Herbe à balais.

AGROSTIDE INTERROMPUE. Agrostis interrupta $L$. Anemagrostis interrupta Trin. Apera interrupta $P . d$. B. Cynosurus splendens $T e n$. Anemagrostis spica renti, var. C. Trin.

AGROSTIDE DU MEXIQUE. Agrostis mexicana $L$. Agrostis cinna Retz. Trichochloa mexicana Trin. Podosemum mexicanum Lapl. Muehlenbergia mexicana Trin. Cinna mexicana Link.

agrostide vUlgaire. Agrostis vulgaris With. A. stolonifera Poll. A. capillaris Schkuhr. A. hispida Wlldw. A. lithuanica Bess. A. rubra Wahl. A. polymorpha Huds. A. vulgaris et divaricata Hoffm. A. verticillata et violacea Thuill. A. frondosa Tenor. Vilfa vulgaris P.d. $B$. 
ALPISTE AQUATIQUE. Phalaris aquatica $L$. P. minor Retz. P. capensis Thunb. N. v. Phalaride aquatique.

ALPISTE DES CANARIES. Phalaris canariensis $L$. P. ovata Moench. N. v. Blé des Canaries. Cunère. Lime. Millet long. Millet plat. Graine d'escayol. G. d'aspic. G. d'oiseau.

ALPISTE RONGÉE. Phalaris præmorsa $L a m$. P. paradoxa $L$. N. v. Phalaride rongée. $P$. incomplète.

aLPISTE ROSEAU. Phalaris arundinacea $\boldsymbol{L}$. Arundo colorata Wlldw. Baldingera colorata $F l$. Wett. Calamagrostis colorata $D$. C. Cal. variegata With. Typhoides arundinacea Mocnch. Baldingera arundinacea $D m i t$. v. v. Fromentau. Herbier. La variété à feuilles panachées est connue sous les noms de: Chiendent ruban. Roseau à feuilles rayées. Roseau panaché. Roseau rayé. Roseau ruban.

AMMOPHILE DES SABLES. Ammophila arundinacea $\boldsymbol{H}$. Calamagrostis arenaria $R$. Arundo arenaria $L$. Psamma littoralis $P . d . B$. Psamma arenaria $R$. el $S$. Phalaris ammophila Link. Phalaris maritima Nutt. Psamma pallida Presl. Ammophila arenaria Link.

ARRHÉNATHĖRE FAUSSE AVOINE. Arrhenatherum avenaceum $P . d . B . A$. elatius $M$. et $K$. Avena elatior $L$. Holcus avenaceus Scop. Avena bulbosa Wlldw. Holcus bulbosus Schrad. Avena precatoria Thuill. Arrhenatherum precatorium Dietr. v. v. Fromental. Avenat. Faux froment. Faux seigle. Fenasse. Pain vin. Raygrass de France. Chiendent à perles. Gros chiendent à crottes. Gros chiendent à troches.

ASPRELLE fAUX RIZ. Asprella oryzoides Lam. Leersia oryzoides Swartz. Phalaris orizoides $\boldsymbol{L}$. Ehrharta clandestina Wigg. Homalocenchrus oryzoides Poll.

AVOINE CARYOPHILlÉE. Avena caryophyllea Wib. Aira inflexa et A. Leersii Lois. Aira divaricata Pourr. Aira canescens Host.

AVOINE COURTE Avena brevis $R$. N. v. Avoine pied d'alouette.

AVOINE CULTIVEEE. Avena sativa $L$. Avena disperma 1 ill. $N$. v. Avoine. Aveine. Avoine noire.

AVOINE ÉlancéE. Avena strigosa Schreb. Danthonia strigosa $P . d . B$. Avena nervosa Lam. N. v. Avoine des Oreades.

AVOINE FOLLE. Avena fatua $L$. N. v. Avron. Avoine bouffe ou bouffle. Averon. Coquiole. Folle avoine.

AVOINE HÉRISSÉE. Avena hirsuta $N$. Avena hirsula $R$.

AVOINE JaUnatre. Avena flavescens $L$. Triselum flavescens $P$. $d$. B.

Trisetum pratense Pers. Trisetum splendens $P$ resl. N. v. Avenette blonde. Avoine blonde. Petit fromental.

AVOINE NUE. Avena nuda $\boldsymbol{L}$.

A VOINE ORIENTALE. Avena orientalis $L$. Avena racemosil Thuill. A. heteromalla Moench. 
Avolne Précoce. Avena pracox $P . d$. B. Trisetum procox Dmrt. Aira subspicala Rainv. Avena pusilla Wreb. Aira præcox $L$. AVOINE DES PRÉS. A vena pralensis $\boldsymbol{L}$. Avena bromoides $\boldsymbol{L}$. AVOINE PUBESCENTE. A vena pubescens $L$. Trisetum pubescens R.et $S$. A. pratensis Gort. A. Canlabrua Lagasc. x. v. Averone. AVOINE tenUe. Avena tenuis Moench. Triselum tenue $R$. ct $s$. Avena dubia Lcers. Avena Iriaristala Vill. Ventenatia bromoïdes et avenacea Koel. Bromus triflorus Poll. Holcus biaristatus Web. Avena striata Lam. Triselum striatum Pers. Avena fertilis All.

BARBON DIGITÉ. Andropogon ischœmum. A. angustifolius Smith et Dmrt. A. villosus Lam. x. v. Brossière. Chiendent à vergetle.

BRIZE A GROS ÉPILLETS. Briza maxima $L$. x. v. Herbe à clochette. Ilerbe à pendants.

BRIZE MOYENNE Briza media $L$. B. Iremula $D . C$. B. virens $L$. B. lutescens Fourc. N. v. Amourette. Crolette. Gramen tremblant. Grolette. Pain d'oiseau. Tamisaille.

BRIZE PETITE. Briza minor $L$. Briza aspera Knapp. v. v. Brize à petites fleurs et à beaucoup de fleurs. Nec plus ultrò des champs.

BROME DES CHAMPS. Bromus arvensis L. B. versicolor Poll. B. multiflorus Weig. B. spiculiteuuata Knapp.

BROME DRESSÉ. Bromus erectus Huds. B. pseudarvensis Koel. B. montanus $F l$. Wett. B. angustifolius Schrank. B. perennis Vill. B. agrestis $A l l$. B. arvensis Poll. Festuca hirta Seen. B. glaucus Lap. Festuca montana Savi. N. v. Brome des prés.

BROME ÉCHANGÉ. Bromus commutatus Schrad.

BROME ÉPAIS. Bromus grossus D.C. B. velutinus Schrad. B. multiflorus Knapp.

BROME EN GRAPPE. Bromus racemosus L. Bromus pratensis Ehrh. B. multiflorus $R$. B. lıordeaceus $L$. B. simplex Gaud. B. gaudini $R$. ct $S$. Brome inerme. Bromus inermis Poll. Fesluca Leysseri Motnch. Festuca speciosa $L$. Schenodorus inermis $R$. et $S$. Festuca inermis $D$. C. Festuca pœoides Thuill. Festuca pæformis Pers.

B ROME LUISANT. Bromus nitidus Dmrt.

BROME MICHEL. Bromus michelianus $N$. B. arduennensis Kunth. Libertia arduennensis $L e j$. Wichelaria bromoïdea $D m \iota r$. Calotheca bromoïllea $L e j$. B. auriculatus $L e j$. B. polystachyus Desf. B. multiflorus, $\beta$ bracteolis auriculatis triaristatis Reichenb. B. triaristatus Lois. BROME MOU OU DOUX. Bromus mollis $L$.

BROME ROIDE. Bromus rigidus $R$. B. rubens Host. B. madritensis Vahl. B. villosus Forsk. B. incrassatus Lam.

BROME RUDE. Bromus asper Murr. B. montanus Scop. Festuca aspera. M. et $K$. B. altissimus Web. B. ramosus. Murr. B. nemorosus Vill. B. hirsutus Curt. B. hirsutissimus Cyrill. 
BROME SEIGLIN. Bromus secalinus L. B. vitiosus Weig. BROME SQUARREUX. Bromus squrrosus $L$.

BROME STÉRILE. Bromus sterilis $L$. B. grandiflorus $\alpha$ Weig. B. distichus Moench.

BROME DES TOITS. Bromus tectorum $L$. B. grandiflorus $\beta$ Weig. B. scabriflorus Opitz.

CALAMAGROSTIDE DES BOIS. Calamagrostis sylvatica $D$. C.C.Arundinacea L. Arundo sylvatica Schrad. Calamagrostis pyramidalis Host. Agrostis villosa Vill. Deyeuxia sylvatica Kunth.

CALAMAGROSTIDE LANCÉOLÉE. Calamagrostis lanceolata $\boldsymbol{R}$. Arundo calagrostis $L$. Arundo leersii Octt. Calam. ramosa Host. Agrostis lanceolata et ramosa $R$. et $S$. Cal. gaudiniana Reich.

CALAMAgrostide DES MONTAgnes. Calamagrostis montana Host. Deyeuxia montana Poir. Arundo montana Gaud. Arundo clarionis Lois. Calamagrostis arundinacea Wib. Arundo varia Schrad. - Deyeuxia varia Kunth.

CAlamagrostide SUBULÉe. Calamagrostis subulata $D \mathrm{~m} t r$. Calam. acutiflora $D$. C. Arundo agrostis Scop. Deyeuxia acutiflora $P$. d. B.

CALAMAgrostide terRestre. Calamagrostis epigejos $R$. Arundo epigejos $L$. Cal. lanceolata With. Arundo canescens Wigg. Ar. lithuanica et velnensis Schult. Cal. lithuanica et velnensis Bess. Ar. intermedia Gmel.

CANCHE CESPITEUSE Aira cespitosa $L$. Campelia cespitosa $L n k$. Aira altissima Moench. Calamagrostis arundo $R$. Aira alpina $R$. A. parviflora Thuill. A. paludosa Wib. A. bottnica Link.

CANCHE DISCOLORE. Aira discolor Thuill. Aira uliginosa Weihe. A. discolor $L e j$. A. paludosa $R$.

CANCHE FLEXUEUSE. Aira flexuosa $L$. A. montana Koel. Avena flexuosa $M$. et $K$.

CATABRose aquatioue, Calabrosa aqualica $B . d . B$. Aira aquatica $L$. Nolinia aquat. Web. Hydrochloa airoïdes Hartm. Poa airoïdes Koel. Glyceria aquatica Smith. Glyceria airoïdes Reichenb. Colpodium aquaticum Trin.

ChIENDENT DACTYLE. Cynodon dactylon Rich. Panicum dactylon $\boldsymbol{L}$. Digitaria stolonifera Schrad. Paspalum umbellatum Lam. Fibigia umbellata Koel. Dactylon officinalis Vill. Paspalum daclylon Lam. Agrostis linearis Retz. Panicum lineare Burm. Digitaria linearis Pers. Cynodon maritimum Iumb. et Kunth. Chloris maritima Trin. Digitaria maritima Spreng. Agrostis bermudiana Tuss. v. v. Chiendent pied de poule. Gros chiendent. Pied de poule.

COKYNÉPhore blanchatre. Corynephorus caneseens $P . d$. $B$. Avena canesecus $W c b$. Weingaertueria canescens Bemp. Aira canescens $L$. 
CYNOSURE CRÉtelLe. Cynosurus cristatus $L$. v. v. Crételle des prés. Crélelle huppée.

DACTYLE GLOMÉRÉ. Dactylis gglomerata $L$. Festuca glomerata All. Dactylis scabra Mann. Bronus glomeratus Scop. x. v. Dactyle pelotonné. Dactyle à paquets.

DIGITAIRE GLABRE. Digitaria glabra $P . d \quad B$. Syntherisma glabrum Schrad. Panicum glabrum Gaud. P. humifusum Kunth. P. lineare Krock. P. ischœmum Schreb. P. arcnarium Bieb. Paspalum ambiguum $D . C$.

DIGITAIRE SANGUINE. Digitaria sanguinalis Scop. D. marginata Link. D. præcox Wlldw. Syntherisma vulgare Schrad. Paspalum sanguinale $\alpha$ Lam. Phalaris velutina Forsh. Panicum sanguinale $\boldsymbol{L}$. Cynodon præcox $R$. et $S$.

ELYME D'EUROPE. Elymus europæus $\boldsymbol{L}$. Triticum sylvaticum Salisb. Secale villosum Huds. Cuviera europæa Koel. Hordeum cylindricum IIurr. II. sylvaticum Vill. H. europæum All. II. montanum Schrnk. H. elymoïdes Vest.

ELYME GÉNICULĖ. Elymus geniculatus Curt.

ELYME DES SABLES. Elymus arenarius $L$. $x$. v. Grand chiendent des mers.

ENODIE BLEUE. Enodium cæruleum $P . d . B$. Nolinia cærulea Moench. Aira cæerulea $L$. Nelica cærulea $L$. Enodium cærulcum Gaud. Molinia varia Schrnk. Aira atrovirens Thuill. Festuca cærulea $D$. C. Hydrochloa cærulea Harlm. Mol. altissima Link. Enodium sylvaticum Link. Mol. arundinacea Schrnk. Mol. littoralis Host. Enodium littorale Reichenb.

ERAGROSTIDE POILU. Eragrostis pilosa $P . d$. B. Poa pilosa $L$.

ERAGROSTIDE VULGAIRE. Eragr'ostis vulgaris Coss. et Germ. Poa megastachya et eragrostis Linn.

FEtTUQUe a UNE PAILletTf. Festuca uniglumis Host. Vulpia membranacea Link. mygalurus uniglumis Link. Vulpia uniglumis Reichenb. Bromus hordeiformis Lam.

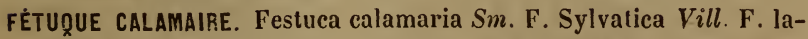
tifolia Host. Poa trinervata Schrad. P. Sylvatica Poll. Schenodorus calamarius $R$. et $S$. Schenodorus sylvaticus Hoppe.

fÉtUQUE faUSSE IVRaIE. Festuca loliacea Huds. Poa loliacea Koel. Fest. elongata Ehrh. Brachypodium loliaceum Link. Fest. phœrix Thuill. Schenodorus loliaceus $R$. et $S$.

FĖTUQUUE GÉANT. Festuca gigantea Vill. Bromus giganteus $\boldsymbol{L}$.

FÉTUQUE HÉTÉROPHILLE OU A FEUILLE VARIÉE. Festuca heterophylla Lam. et Haenck. F. nemorum Leyss. F. duriuscula $L$. fÉTUQUe POLYMORPHE. Festuca polymorpha Nob. Festuca ovina $L$. F. glauca Schrad. et Lam. F. tenuifolia Sibthorp. F. guestfalica Boen- 
ningsh. F. pallens Host. Poa capillata Mérat. Festuca capillata Lam. F. valesiaca Gaud.

FÉTUQUE DES PRÉS. Festuea pratensis $\boldsymbol{H} u d s$. Schenodorus pratensis $\boldsymbol{P}$. d. B. Bromus pratensis Spreng. B. elatior Koel. Poa curvata Koel.

FÉTUQUE QUEUE D'ÉCUREUIL. Festuca sciuroides $R$. Vulpia seiuroides et Bromoides Reichenb.

FÉTUQUE QUEUE DE SOURIS. Festuca preudomyuros Soy-wllmt.

FÉTUQUE ROSEAU. Festuea arundinaeea $S c h r e b$. F. elatior $L$. Sehenodorus elatior $\boldsymbol{P}$. $d$. B. Bromus arundinaceus $R$. Br. elatior Spreng. Schenolorus arundinaceus $D m r^{`} t$. s. v. Fétuque élevéc. Grande fétuque. Grande queue de rat.

FÉtUQUE ROUgE. Festuca rubra $L$. F. dumetorum $L$. barbata F. Schrnk. F. cinerea $D$. C. F. villosa Bacr. N. v. Fétuque traęante.

FLÉOLE DES ALPES. Phleum alpinum $L$. P. pratense Schreb. P. commutatum Gand.

FLÉOLE APRE. Phleum asperum Vill. Phalaris aspera Relz. Chilochloa aspera $P . d . B$.

FLÉOLE HÉRISSÉE. Phleum hirsutum Sut. P. michelii All. P. phalaroideum Vill. Phalaris alpina Haenke. Chiloehloa michelii P. d. B.

FLÉOLE LISSE. Phleum læve Bieb.P. Boemeri $W i b$. Phalaris phleoides $L$. Chilochloa Bochmeri $P$. d. B.

FLÉOLE DES PRÉS. Phleum pratense $L$. P. nodosum $L$. P. arvense Pourr N. v. Grosse massette. Manette. Marsette. Massette des prés. ThimoThimothy. Queue de rat.

FLÉOLE DES SABLES. Phleum arenarium $\boldsymbol{L}$. Phalaris arenaria Wllnw. Phalaris phleoides $\beta$ Ait. Crypsis arenaria Desf. Chilochloa arenaria P.d. B.

FLOUVE ODORANTE. Anthoxanthum odoratum $L$.

FROMENT AMIDONNIER. Triticum amyleum Ser. dicoccum Schrnk. T. spelta IIost. T. gaertnerium et cienfugos Lag. T.atratum Host. T. tricoecum Schuebl. N. v. Blé amidonnier. Froment amylacé.

FROMENT DES BOIS. Triticum sylvatieum Moench. Bromus sylvaticus Poll. B. pinnatus $\beta$ Tin. Festuca sylvatica Huds. Brachypodium sylvaticum et gracile $R$. et $S$.

FROMENT DES CHIENS. Triticum caninum Schreb. T. sepium Lam. Elynus eaninus $L$. $\Lambda$ gropyrum eaninum $R$. ct $S$. Festuca nutans Moench.

FROMENT CILIÉ. Tritieum ciliatum D. C. Brachypodium distachyon $R$. ct $S$. Traehyuia distachia $L i n k$.

FROMENT CULTIVÉ. Triticum sativum Lam. T. vulgare Vill. T. æstivum et hyberuum $\boldsymbol{L}$. . v. blé d'hiver et de mars. Blé. Bled.

FROMENT ENGRAIN. Triticum monoccoccum $L$. v. v. Blé locular. Epeautre locular. Petile épeautre. Riz sec. 
froment ÉPEaUtre. Triticum spelta $\boldsymbol{L}$. T. Zea Host. N. v. Grande épeautre, Ampeutre. Engrain. Froment rouge. Ingrain. Spaute. Spautre.

FROMENT JONCIFORME. Triticum junceum Smith. Agropyrum junceum $P$. d. B. T. glauceum D. C. v. v. Chiendent sans pareil.

froment naro. Triticum uardus D. C. Festuea tenuiflora Schrad. Nardurus tenellus Rcichenb.

FROMENT PINNÉ. Triticun pinnatum .Moench. Bromus pinnatus $\boldsymbol{L}$. Festuca pinnata Mocnch. Bromus corniculatus Lam. Brachypodium pinnatum $\boldsymbol{P}$. $d$. B. s. v. Jaucon. Palène. Paulène. Pimouche.

FROMENT DE POLOGNE. Triticum polonicum $L$. T. glaucum Mocnch. T. cevallos Lagas. Agropyrum lævissimum $\boldsymbol{P}$. $d$. B. x. v. Seigle de Pologne. Seigle de Russic.

FROMENT ROIOE. Triticum rigidum Lej. Agropyrum rigidum Dmrt. FROMENT TRAÇANT. Triticum repens Smith. v. v. Agrum. Auge. Chiendent des boutiques. Laitue de chien. Petit ehiendent. Sainte neige.

froment tURgide. Triticum turgidum $L$. Triticum durum Desf. Trit. excelsum Duchesnc. v. v. Blé barbu. Gros blé. Petanielle rousse. Blé gẻant. Blẻ de Sainte. IIélène. Blé glacé. Blé corné.

GASTRIDIE LENDIGĖRE. Gastridium lendigerum $P . d . \quad B$. G. australe $P$. $d$. B. Milium lendigerum $L$. Agrostis panicia Lam. Calamagrostis schwabii Spreng. Gastridier lendigère.

GLYCÉRIE AQUATIQUE OU ÉLEVÉE. Glyceria spectabilis $M$. et $K$. G l.aquatica $S m$. Poa aquatica $L$. P. altissima Moench. Hydrochloa aquatica Hartm. s. v. Fécondité des marais.

GLYCÉRIE DISTANTE. Glyceria distans Wahl. Festuca distans Kunth. Poa distans L. P. Salina Poll. P. retroflexa Cur'l. P. arenaria Retz. Hydrochloa distans Hartm.

gLYCÉRIE flottante. Glyceria fluitans $B r$. Festuca fluitans $L$. Poa fluitans Scop. Hydrochloa fluitans $I I a r l m$. Poa dulcis Clarv. v. v. Banoue. Chiendent de la manne. Chiendent flottant. Fétuque penehée. Ilerbe à la manne. Manne aquatique. Manne d'Allemagne. Manne de Pologne. Manne de Prusse. Paturin.

GLYCÉRIE MARITIME. Glyecria maritima $\boldsymbol{M}$. e $\mathrm{K}$. Poa maritima Il $u d s$. P. thalassica Kunth. Poa arundinacea IIocneh. Ifydrochloa maritima Hartm.

HIEROCHLOE AUSTRALE. IIierochloë australis $R$. et $S$. II. odorata $P . d . \boldsymbol{B}$. Holcus australis Schrad. Holcus odoratus Schkuhr. Avena odorata var. $\alpha$ D. C.

HIEROCHLOE BORÉALE. Ilierochloë borealis $R$. et $S$. II. odorata Wrahl. II. repens $\boldsymbol{P} . \boldsymbol{d}$. B. IIoleus odoratus Schkuhr. Avena odorata Pers. HOUQUE OU HOULQUE LaINEUSE. IIolcus lanatus $L$. Avena lamata Kocl, x. v. Blanchard veloutć. Houlque arisléc. 
HOUQূUE MOLLE. Holcus mollis $L$. Avena mollis Koel. Aira mollis Schreb.

IVRAIE DES CHAMPS. Lolium arvense With.

IVRAIE ÉNIVRANTE. Lolium tenulentum $L$. L. annuum $L a m$. Crepalia temulenta Schrank. L. speciosnm Sicv. L. robustum Reichenb. x. v. Grol. Ilerbe d'ivrogne. Ivraie. Jucil. Pimouche. Orge Zizanie. IVRaIE D'ITALIE. Lolium italicum $N$. L. boucheanum Kunth. IVRAIE RIEFFEL. Lolium raffelianum $L$. L. multiflorum Lam.

IVRAIE VIVACE. Lolium perenne $\boldsymbol{L}$. L. tenue $\boldsymbol{L}$. x. v. Bonne herbe. Fausse ivraie. Fromental d'Angleterre. Gazon anglais. Ivraie de rat. Jaucon. Lai. Lolie, Margau. Pain Vin. Palisse. Pimouche. Ray-grass d'Angleterre.

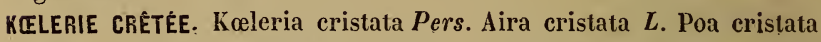
With. Dactylis eristata Bieb. Festuca cristata Vill.Airochloa cristata Link. Festuca dactyloides $R$. Dactylis caudata Brot.

LAGURIER OVALE. Lagurus ovatus $L$.

LASIAGROSTIDE CALAMAGROSTIDE. Lasiagrostis calamagrostis Link. Agrostis calamagrostis $\boldsymbol{L}$. Arundo speciosa Schrad. Calamagrostis argentea $D$. C. S. speciosa Host. Stipa calamagrostis Wahl. w. v. Calamagrostide argentée.

LEPIURE ARQUÉ. Lepiurus incurvatus Dmtr. Ophiurus incurvatus $R$. Lepiurus incurvatus Trin. Rotljollia incurvata $L$. OEgylops incurvata $L$. Agrostis ineurvata Scop. x. v. Herbe à serpents.

LEPIURE ÉLANCÉ. Lepiurus strigosus $\boldsymbol{D} m \iota r$. Lepiurus filiformis Trin. Ophiurus filiformis $R$. et $S$. Rottboellia erecta Savi. Rottboeilia incurvata $\beta$ D. C.

MAIS CULTIVÉ. Maïs sativa Tournef. Zea mays $L$. Naïs americana Baunig. Maïs zea Gacmnt. v. v. Blamarié. Blé de Barbarie. Blé d'Espagne. Blé de Guinée. Blé d'Inde. Blé d'Italie. Blé de Turquie. Froment des Indes. Garouilhe. Graine de Turquie. Gros millet des Indes. Maiz. Mahiz. Nillaral. Nillargo. Millargou. Troquet, Turquet. Turquie.

MÉLIQUE CILIÉE. IIclica ciliata $\boldsymbol{L}$.

MÉLIQUE ÉLEVÉE. Helica altissima $L$. M. sibirica $L a m$.

MÉLIQUE PENCHÉE. Melica nutans $\boldsymbol{L}$. NI. montana IIuds.

MÉLIQUE UNIFLORE. Melica uniflora Retz. M. Lobelii Vill. M. nutans Lam.

MIBORE NAINE. Mibora minima Dmtr. MI. verna Adans. Chamagrostis minima Borkh. Knappia agrostidea Smith. Kn. verna Trin. Sturmia minima Hoppe. St. verna Pers. Agrostis minima $L$.

MILLET OU MILIER ÉPARS. Milium effusum L. Agrostis effusa D. G. Miliarum effusum Mloench.

NARD ÉLANCÉ OU SERRÉ. Nardus stricta $\boldsymbol{L}$. 
EEGYLOPE ALLONGE. OEgylops triuncalis $L$.

EEGYLPE OVALE. OEgylops ovata $\boldsymbol{L}$. Phleum ngylops Scop.

OPLISMĖNE PIED DE COQ. Oplismus crus galli Kunth et Dmit. Panicum crus galli $L$. Pan. crus corvi $L$. P. zonale Guss. Echinochloa erus galli $P$. $d . B$. Eehin. crus corvi $P$. d. B. Milium erus galli \#oench. x. v. Crète de coq, Ergot de coq. Millard. Patte de poule. Panis des marais.

ORGE BULBEUSE. IIordeum bulbosum $L$. II. ciliatum Lam. II. strictum Desf. II. nodosum Savi.

ORGE COMMUNE IIordeum vulgare $\boldsymbol{L}$. $x$. v. Escourgeon.

ORGE DISTIQUE. Hordeum distichum $L$. Zeocriton disticlium $P$. $d . B$. x. v. Baillarge. Orge à longs épis. Orge anglaise. Orge distique. Paumelle. Paoumoule. Petite orge. Poumelle. Sucrion. Pamelle.

ORGE MARITIME. Hordeum maritimum Valh. et Wilh. H. rigidum $R$.

II. geniculatum All. II. manicum Huds. Zeocriton maritimum P. $d$. B.

ORGE DES PRĖS. IIordeum pratense Huds. II. secalinum Schrad. H. nodosum $L$. Zeocriton secalinum $P$. $d . B$.

ORGE PYRAMIDALE. Hordeum zeocriton $L$. Zeocriton commune $P$. $d$. $D$. H. distichum $\beta$ Lam. x. v. Faux riz. Froment barbu. Orge de Russie. Riz d'Allemagne. Riz rustique.

ORGE A SIX RANGS. Hordeum hexastichum $L$. x. v. Escourgeon. Orge anguleuse. Orge d'Achille. Orge de prime. Orge d'hiver. Orge carrée. ORGE DES SOURIS Hordeum murinum $L$. Zeocriton murinum $P$. $d . \boldsymbol{B}$. N. v. Orge queue de souris.

PANIS CAPILLAIRE. Panicum capillare $L$. P. strigosum Muchl.

PANIS ÉLEVÉ. Panicum altissimum Vill. P. jumentorum Hich. P. læve. Lam. P. maximum Jacq. P. Polygamum Swartz. N. v. Herbe de Guinéc.

PANIS MILLET. Panicum miliaceum $L$. P. milium Pers.

PATURIN DES ALPES. Poa alpina $L$. P. vivipara Wllndw. P. brevifolia D. C. P. Badensis Haenke. P. Cenisia Link. P. collina Host.

PATURin ANNUEl. Poa annua. $L$. v. v. Gazon éternel.

PATURIN BleUATRE. Poa cæsia Sm. et Dmrt.

PATURIN DES BOIS. Poa nemoralis $\boldsymbol{L}$.

PATURIN BULBeUX. Poa bulbosa $L$. P. prolifera Sclimidl. P. crispa Thuill. x. v. Paturin échalote.

Paturin commun. Poa trivialis $L$. P. pratensis Poll. P. dubia $L c e r s$. x. v. Paturin roide.

PATURIN COMPRIMÉ. Poa compressa $L$. P. muralis Wib. x. v. Paturin. IIerbe des murs.

PATURIN fertile. Poa fertilis $I$. P. palustris $R$. P. serotina Gaud. P. effusa Kit. P. anģustifolia Wrah!. 
PATURIN DES PRÉS. Poa pratensis $L$. P. trivialis Leyss.

PATURIN DE SILÉSIE. Poa sudetica Haencke. P. rubens Moench. P. sylvatica Vill. P. latifolia Pohl. P. trinervata D. C. P. lithuanica Gorski.

PIPTATHĖRE MULTIFLORE. Pipthatherum multiflorum $P$. $d$. B. Nilium multiflorum Cav. Agrostis miliacea $L$. Nilium arundinaceum Sibth. Urachne multiflora et frutescens Link. Urachne parviflora Trin.

POLYPOGON DE MONTPELLIER. Polypogon monspelliensis Desf. Santia plumosa Savi. Phalaris cristata Forsk. Alopecurus monspelliensis $\boldsymbol{L}$. Al. aristatus $\boldsymbol{H} u d s$. Al. paniceus $\boldsymbol{L}$. Agrostis panicea Wlldnw. Ag. triaristata Knapp. Ag. alopecuroïdes Lam. Ag. crinita Moench. Phleum crinitum Schreb. Phleum monspelliense Koel. Polypogon paniceum Lagas. Polypogon crinitum N'ult. Cynosurus paniceus $\boldsymbol{L}$.

POLYPOGON DES RIVAGES. Polypogon littoralis $\mathrm{Sm}$. Agrostis littoralis With. Ag. lutosa Poir. Polypogon elongatus Lagas. Polypogon lagascæ $R$. et $S$.

ROSEAU A BALAIS. Arundo phragmites $L$. Phragmites communis Trin. Arundo vulgaris Lam. v. v. Cannette. Roseau aquatique. Roseau des marais. Roseau. Rouzeau.

SCLÉROCHLOE COUCHÉ. Schlerochloa procumbens $P . d . B$. Festuca procumbens Kunth. Poa procumbens Curt. Glyceria procumbens Smith.

SCLÉROCHLOE ROIDE. Sclerochloa rigida Link. Festuca rigida Kunth. Glyceria rigida $S m$. Poa rigida $L$.

SEIGLE CULTIVÉ. Secale cereale $\boldsymbol{L}$.

SESLÉRIE BLEUE. Sesleria cærulea $A \boldsymbol{r} d$. Cynosurus cæruleus $\boldsymbol{L}$. Aira varia Jacq.

SÉtaire glauQue. Setaria glauea $P . d . B$. Panicum glaucum Nees ab Esenb.P. flavescens Moench. P. lutescens Wcig. P. lævigatum $\beta$ Lam. P. puncilum $\boldsymbol{P}$ oir. Setaria pumila $R$. et $S$. Pennisctum glaucum $\boldsymbol{B} r$.

SÉtalre d'ItAlie. Setaria italica $P$. $d$. B. Panicum italicum $L$. P. erythrospermum Hornem..P. Setosum Lam.

Cette espèce comprend trois variétés basées sur des caractères peu variables qui sont connues sous les noms suivants :

10 Germanica $R$. Breviseta lutescens ou flavescens $N o b$. N. v. Millet de Hongrie. Moha.

$2^{\circ}$ Setosa $R$. et $S$. Longiseta flavescens $N^{\prime} o b$.

$3^{\circ}$ Marilima Michel Lej. Longiseta purpurescens Nob.

SÉTAIRE VERTE, Setaria viridis $P . d . B$. Panicum viride $L$. P. bicolor Moench. Pennisctum viride $B r$. P. lævigatum a Lam. N. v. Murge. Panis lisse. Panis sauvage. Penessic.

SÉTAIRE VERTICILLÉE. Setạia verticillata $P$. $d . B$.Panicum verticillatum $\boldsymbol{L}$. Panicum asperum Lam. Penniselum verticillatum $\boldsymbol{B} r$.

SORGHIER VULGaIRE. Sorghum vulgare $P$ ers et Mich. Holcus sorghum $L$. Ilolcus durra Forsh. Andropogon sorghum Brot. v. v. Balais. Balai. 
de jonc. Blé de Guinée. Granotte. Grand ou gros mil. Grand millet noir. Houque à balais. Maĩs de Guinéc. Nil d'Éthiopie. Mil d'Inde. Millauge. Milliage. Millet a balais. Millet d'Afrique. Millet de Turquie. Sorgho. Sorgho d'Afrique.

STIPE CAPILLAIRE. Stipa capillata $L$. S. juncea $L a m$.

STIPE EMPENNÉ. Stipa pennata $L$. x. v. Stipe à panache. Etiçpe aigrette.

TRAGIER EN GRAPPE. Tragus racemosus Hall. Lappago racemosa Wlldnw. Cenchrus racemosus $L$. C. linearis Lam. Phalaris muricata Forsh.

TRIODIE INCLINÉE. Triodia decumbens $P . d$. B. Danthonia decumbens $D$. C. Poa decumbens $W$ ith. Festuca decumbens $L$. Bromus decumbens Koel. Sieglingia decumbens $B e r n h$. Nelica rigida Wib. Melica decumbens $W e b$.

VULPIN BULBEUX. Alopecurus bulbosus $\boldsymbol{L}$.

VULPIN DES CHAMPS. Alopecurus agrestis L. Al. myosuroides Hu!s. Pleum flavum Scop. x. v. Vulpin agreste. Queue de renard des champs. Racourt.

VULPIN FAUVE. Alopecurus fulvus $S m$. Al. paludosus $P$. $d$. B. Al. geniculatus Poll. Al. aristatulus .IFich. Al. subaristatus Pursh.

VULPIN DES PRÉS. Alopecurus pratensis $L$. Al. nigricans Hornem. x. r. Chiendent queue de renard. Queue de renard des prés.

VULPIN UTRICULÉ. Alopecurus utriculatus Pers. Phalaris utriculata L. Tozzettia utriculata et pratensis Savi. Cornucopia alopecuroides $L$. 


\section{TABLE \\ DES TERMES TECHNIQUES.}

\section{PREMIÈrE PARTIE.}

Acicule ........

Aciculiforme ......

Aculéiforme (sub) . . .

Acuminé.

Aérien.

Aigu.

Ailé.

Alterne . . . . . . .

Incipité. . . . . . .

Annuel . . . . . . .

Anthère.

Apical (sub)

Arêle

Aristé.

Arrondi.

Articulé.

Axe

Axillaire

Bâle.

Barbe.

Basilaire.

Bifide.

Bipaléolé

Bipartite.

Bipplié ...

Bisannuel.

Bractée.

Bulbe

Caduc.

Calus . . . . . . . . .

Capité.

Caréné.

Caronculé.

Caryopse.

Cassant.

Caulinaire.

Chaume.

Chiendent.

Cilié.

Claviforme

Coléorhize

Compose .

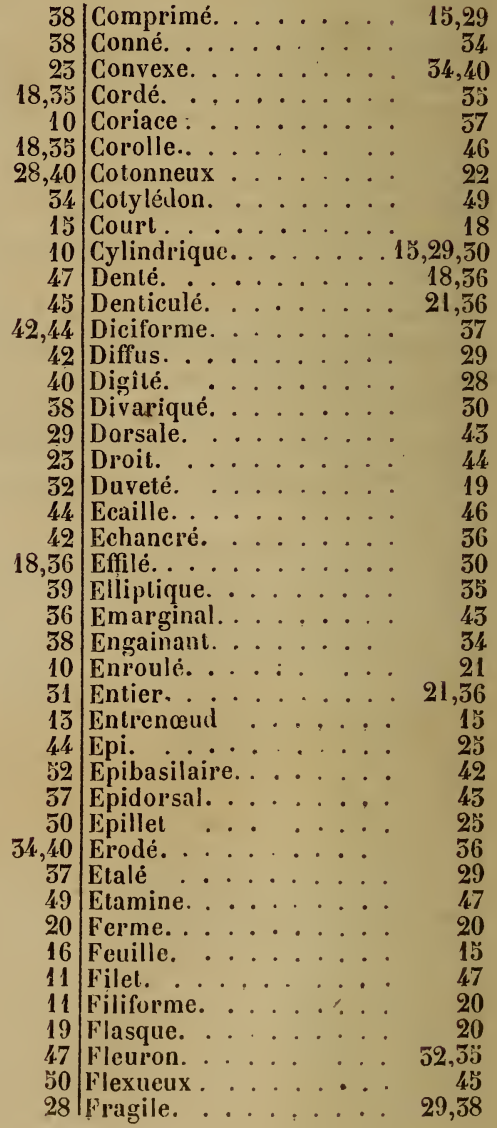

15,29

18,36

28 fragile. . . . . . . 29,38 
Gaine

49 Pauciflore.

Géminé

Gemmule

Genouillé.

Glabre.

Glauque.

Glomerule.

34 Pectiné

49 Pédicelliforme. . . . . 58,46

43 Penicilliforme . . . . 46

22 Persistant. . . . . 29, $28,4,4$

Glume. . .

22 Phycostème. . . . . . 52

52 Pileux. . . . . . . 18

52 Plié.......... 21

extericurc

Glumellule . . . .

52 Poiln.

20,22

52.59 Polygame. . . . . . 51

4.5 Pubescent . . . . . . 22

Grain. . . . . . . .

Herbacé.

49 Pyramidal. . . . . 50

Hermaphrodite. . . . .

Hispide. . . . . . . .

Hypoblaste. . . . . .

Inéquilatère.

Inflorescence

Involucelle.

57 Racémiforme. : . . . 29

51 Rachis. . . . . . . 28

22 Racine. . . . . 10

49 Radical. . . . . . . 16

5 ว̆ Rameux . . . . . 10

25 Réceptacle. . . . . 28

59 Réfléchi . . . . . 29

Involucre. . . .

Irrégulier. . . . . . . . .

Laciniẻ

52 Régulier. . . . . . . 29

29 Rhizome. . . . . . . . 11

18 Roide ... . . . . . 20,37

Laineux. . . . . . . .

Lame.

22 Rubanaire. . . . . . 20

16 Rude. . . . . . . 22

3 ă Rudiment. . . . . . . $\mathbf{5 8 , 4 6}$

Lancẻolé. ......... .

Languette. . . . . . .

Libre.

Ligule.

18 Scabre.

58,46

34 Scarieux. . . . 57

16 Scobine. . . . . . 37

16 Sétacé. . . . . . . 20

Linéaire . . . . . . .

Locuste. . . . . . .

Lodicule. . . . . . . . .

Membraneux.

55 Sillonné . . . . . 21

24,30 Simple. . . . . . 10,2ă

46 Soie ....... 41

Nérithalle. . . .

Mixte. . . . . . .

Mou. . . . . . . .

Mlucroné. . . . . . .

Multiflore.

Mutique .

Neclaire.

18,57 Solide.

29

15 Souterrain. . . . . 10

20 Soyeux. . . . . . 22

20 Spiciforme . . . . . 50

18,3 s̆ Stigmate. . . . . . 48

50 Strié. . . . . . . 21

44 Style . . . . . . 48

46 Suborbiculé. . . . . . 41

Nentre. . . . . . .

Nœud.

31 Subulé . . . . . . כ כ

15 Terminal. . . . . . 25

Nu.

19,59 Tẻtragone. . . . . . . 15,29

oblong. . . . . .

obovale. . . . . .

Obtus. . . . . . . .

Opposé...........

Orbiculè.

Ovaire.

Ovale.

18,3 ă Tige.

3 To Tordu. . . . . 44

19,3̈ Tronqué. . . . 19,50

3 unni......... 22

34 Uniflore. . . . . 51

48 Unilatéral. . . . . 29

54 Unipaléolé. . . . . 59

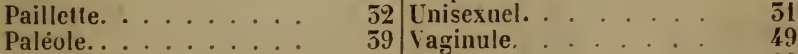

Paléoliforme. . . . . 46 Velu. . . . . . 22

Paléolule.

1.6 Vivace.

29 Zig zag .

Panicule. 


\section{TABLE ALPHABÉTIQUE DES MATIÈRES.}

Trilus, sous-tribus, genres, espèces et variétés.

\section{$2^{\mathrm{e}}$ ET $3^{\mathrm{e}}$ PARTIES.}

\begin{tabular}{|c|c|c|c|c|c|c|c|c|c|}
\hline & 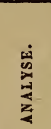 & 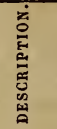 & 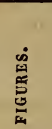 & 这 & & 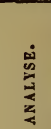 & 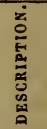 & 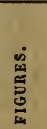 & 崫 \\
\hline Aelbroeckie. & $\mathbf{5} 6$ & 1341 & 134 & & Ammophile. & 59 & 192 & 192 & \\
\hline - maritime. & & 1362 & 283 & 282 & - des sables. & & 193 & & 315 \\
\hline Agrostide. & 59 & 1351 & 185 & 283 & Ammophilees. & 180 & 190 & & \\
\hline - blanche. & 186 & 1893 & 313 & 312 & Andropogonées. & 218 & 231 & & \\
\hline - blanche,̧̧variété géant. & & 190 & & & Anthoxanthées. & 172 & 177 & & \\
\hline - blanche, variété mari- & & & & & Arrhenatheracées. & 51 & 172 & & \\
\hline time. & & 190 & & & Arrénathère. & 58 & 173 & 173 & \\
\hline $\begin{array}{l}\text { - blanche, variété } \\
\text { stolonifère. }\end{array}$ & & 189 & & 312 & $\begin{array}{l}\text { - fausse avoine. } \\
\text { - fausse avoine à une }\end{array}$ & & 174 & 505 & 304 \\
\hline - blanche, variété & & & & & arète. & & 174 & & \\
\hline traçanle. & & 189 & & & - fausse avoine bulbeuse. & & 174 & & 505 \\
\hline - chiens (des). & 186 & 190 & & 514 & - fausse avoine élevée. & & 174 & & \\
\hline - épi du vent. & 186 & 187 & & 312 & - fausse avo & & 174 & & \\
\hline - Interrompu. & 186 & 187 & & 312 & Arrhenathérees. & 172 & 173 & & \\
\hline - Mexique (du). & 186 & 188 & & 312 & Arundinees. & 103 & 148 & & \\
\hline - vulgaire. & 186 & 188 & 313 & 312 & Asprelle. & 60 & 202 & 202 & \\
\hline - vulgaire, var. des & & 189 & & & - faux riz. & & 202 & & $1 \mathrm{tis}$ \\
\hline - vulgaire, var. jat & & 189 & & & Asprellees. & 201 & 202 & & \\
\hline Agrostidées. & 180 & 181 & & & A vénacées. & 57 & 157 & 158 & \\
\hline Agrostidicées. & 59 & 180 & & & Avoine. & 58 & 159 & 160 & \\
\hline Alopécurées. & 201 & 211 & & & - caryophilléc. & 161 & 169 & & 302 \\
\hline Alpiste. & 61 & 204 & 205 & & - courle. & 161 & 164 & 500 & 299 \\
\hline - aquatique. & 206 & 207 & & 319 & - cultivée. & 161 & 162 & 300 & 297 \\
\hline - Canaries (des). & 206 & 207 & 320 & 319 & - cultivée de Brie. & & 164 & & 297 \\
\hline - rongèe. & 206 & 207 & & 319 & - cultivé blanche & & & & \\
\hline IL roseau. & 206 & 207 & 318 & 317 & des Flandres. & & 163 & & 297 \\
\hline
\end{tabular}




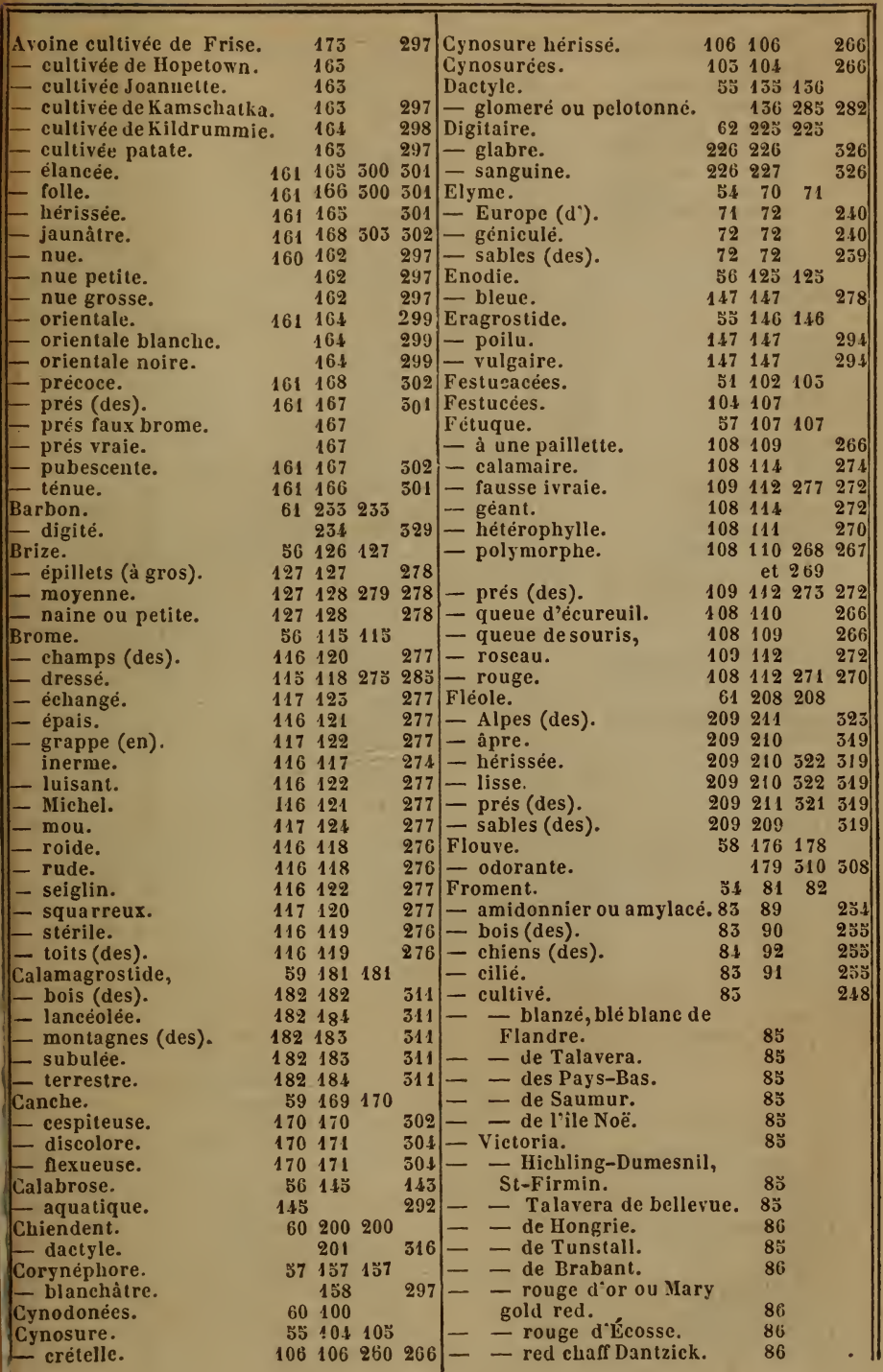




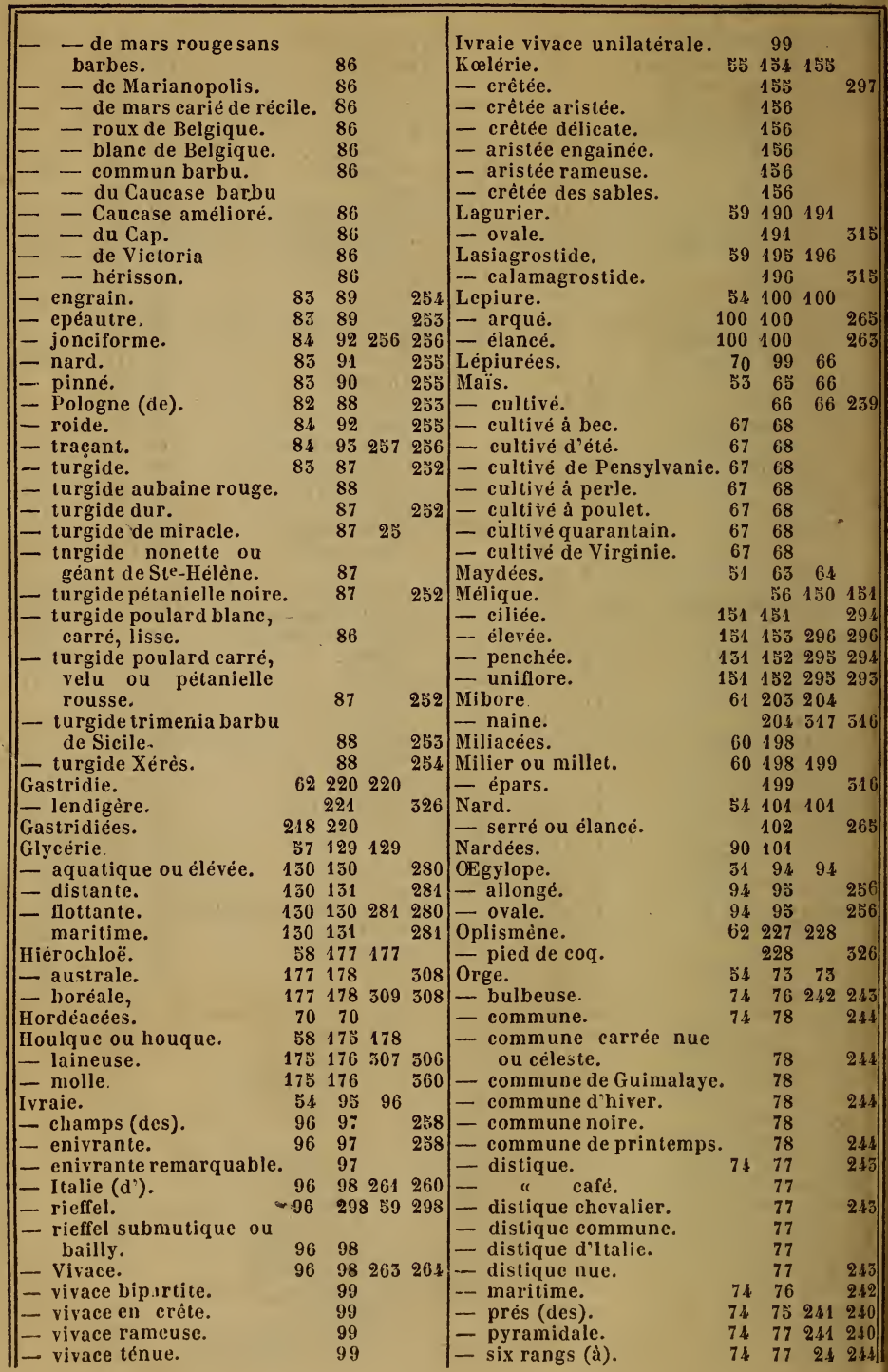


TABLE ALPHABÉTIQUE DES MATIERES.

\begin{tabular}{|c|c|c|c|c|c|c|c|c|c|}
\hline Orge des souris. & 74 & 75 & & 240 & Sclérochloë. & 57 & 152 & 133 & \\
\hline Panicacées. & 52 et 53 & 218 & & & - couché. & 132 & 133 & & 282 \\
\hline Panicées. & 218 & 221 & & & - roide. & 132 & 135 & & 282 \\
\hline Panis. & 62 & 229 & & & Seigle. & 5.1 & 79 & 79 & \\
\hline - capillaire. & 230 & 231 & & 329 & - cultive. & & 80 & & 246 \\
\hline - élevé. & 230 & 251 & & 529 & - cultivé d'hiver ordi- & & & & \\
\hline - millet. & 250 & 251 & 328 & 326 & naire. & & 80 & & \\
\hline Paturin. & 56 & 156 & 157 & & - cultivé de mars. & & 80 & & \\
\hline - Alpes (des). & 158 & 159 & & 286 & - cultivé multicaule. & & 80 & & \\
\hline - annuel. & 138 & 138 & 286 & 284 & - cultivé de Ronie. & & 81 & 249 & 2.4 \\
\hline - bleuàtre. & 138 & 142 & & 290 & - cultivé de Russie. & & 80 & & 217 \\
\hline - bois (des). & 158 & 140 & 288 & 287 & - cultivè de Vierlande. & & 80 & & \\
\hline - bois (des) ferme. & & 141 & & & Seslérie. & 55 & 156 & 156 & \\
\hline - bois (des) glauces & cent. & 111 & & & - bleue. & & 157 & & 297 \\
\hline - bois (des) des I & mon- & & & & Sesléricées. & 10.1 & 154 & & \\
\hline lagnes. & & 141 & & & Sét: & 62 & 221 & 222 & \\
\hline - bois (des) noueux & & 140 & & & $-\mathrm{gla}$ & 222 & 223 & & 526 \\
\hline - bois (des) resserr & & 141 & & & - d'Italie. & 223 & 224 & 327 & 326 \\
\hline - bois (des) subuni & flore. & 140 & & & - ve & 223 & 223 & & 326 \\
\hline - bois (des) vulgair & & 141 & & & cillée. & 222 & 223 & & 326 \\
\hline - bulbeux. & 138 & 139 & & 287 & Sorg & 61 & 232 & & \\
\hline - commun. & 158 & 142 & 291 & 290 & - vulgaire. & & 232 & 232 & 329 \\
\hline - comprimé. & 138 & 141 & & 292 & Stip & $52-53$ & 193 & & \\
\hline - fertile. & 158 & 141 & 289 & 292 & Stipe. & $59-60$ & 193 & 194 & \\
\hline - prés (des), & 138 & 143 & 295 & 287 & - chevelu. & 195 & 195 & & 315 \\
\hline - - ancipité. & & 144 & & & $-\mathrm{e}$ & 19 วั & 195 & & 315 \\
\hline - - élancé. & & 144 & & & trage. & 61 & 219 & 219 & \\
\hline - - feuilles étroit & es (a). & 144 & & & $-\mathrm{e}$ & & 220 & & 326 \\
\hline - - feuilles larges & $s(\dot{a})$. & 143 & & & Tras & 218 & 219 & & \\
\hline - - glauque. & & 144 & & & Trio & 56 & 153 & 153 & \\
\hline - Lejeune (de), & & 143 & & & - inclinee ou penchee. & & 154 & & 296 \\
\hline - - vulgaire. & & 143 & & & Triti & 54 & 68 & 69 & \\
\hline - Silésie (de). & 137 & 144 & & 292 & Triticées. & 70 & 78 & & \\
\hline Phalaridacees. & 52 et 53 & 201 & & & Vulp & 61 & 211 & 202 & \\
\hline Phalaridées. & 201 & 203 & & & $-\mathrm{b}$ & 213 & 213 & & 325 \\
\hline Piptathère. & $59-60$ & 197 & 197 & & - champs (des). & 213 & 214 & & 325 \\
\hline - multiflore. & & 197 & & 515 & - fauve. & 213 & 215 & 321 & 325 \\
\hline Poacées. & 101 & 153 & & & $-g e$ & 213 & 215 & 324 & 520 \\
\hline Polypogon. & 60 & 216 & 216 & & - prés (des). & 213 & 213 & 323 & 323 \\
\hline - Montpellier (de). & 217 & 217 & & $32 \%$ & - prés (des) aquatique. & & 214 & & \\
\hline - rivages (des) & 217 & 217 & & 525 & - prés (des) pẩle. & & 214 & & \\
\hline Roseau. & 53 & 148 & 149 & & - prés (des) tondu. & & 214 & & \\
\hline - & & 149 & & 29 & $1-u$ & 213 & 214 & & \\
\hline
\end{tabular}

FIN DE LA TABLE ALPHABÉ TIQৃUE DES MATIĖRES. 


\section{FAUTES ESSENTIELLES A CORRIGER.}

\begin{tabular}{|c|c|c|c|}
\hline $\begin{array}{l}\text { PAGE. } \\
10 .\end{array}$ & $\begin{array}{l}\text { LiGNe. } \\
11 .\end{array}$ & $\begin{array}{l}\text { AU LIEU DE : } \\
\text { consistante }\end{array}$ & $\begin{array}{l}\text { Lisez: } \\
\text { consistance }\end{array}$ \\
\hline 21. & 12. & F bis $\mathbf{I}$. & E 1 . \\
\hline 34. & 6. & D. & L. \\
\hline 36. & 20. & aristées, & aristé, \\
\hline 37. & 4. & le lancéolé, & le-lancéolé, \\
\hline 58. & 27. & Arrhénathérèes. & Arrhẻnatère. \\
\hline 71. & 6. & l'autre & autre qui est \\
\hline 71. & 7. & sillon & sillon: \\
\hline 83. & 20. & Engrains. & Engrain. \\
\hline 89. & 16. & barbue. & barbu. \\
\hline 92. & 31. & à rejets & des rejets \\
\hline 93. & 4. & paléoles & paléole externe \\
\hline 103. & 10. & munies & munis \\
\hline 116. & 7. & largeur & longueur \\
\hline 117. & 23. & externe & interne \\
\hline 122. & 12. & externe... interne & interne... externe... \\
\hline 139. & 21. & pubescentes & pubescents \\
\hline 139. & 22. & liées & liés \\
\hline 141. & 31. & $4-10$ & $4-10$ \\
\hline 141. & 33. & a & la \\
\hline 143. & 20. & la & le \\
\hline 146. & 5. & $3-5$ & a $3-5$ \\
\hline 148. & 19 et 25 & inférieure & inférieur \\
\hline 148. & 20. & pourvues & pourvus \\
\hline 148. & 25. & dépourvue & dépourvu \\
\hline 148. & 26. & entourées chacune & entourés chacun \\
\hline 150. & 14. & enıbrassés & embrassées \\
\hline 166. & 30. & biaristées & biaristés \\
\hline 166. & 30. & pourvues & pourvus \\
\hline 168. & 7 et 8. & feuilles & lames \\
\hline 177. & 11. & paléoles & paléolules \\
\hline 186. & 24. & chènes. & chiens. \\
\hline 204. & 8. & chaume & chaumes \\
\hline 211. & 28. & réduite d & réduite souvent à \\
\hline 218. & 24. & stérile & stérile; \\
\hline 232. & 9. & ; hyaline & , hyaline; \\
\hline
\end{tabular}




\title{
V. DEMOOR
}

PRAIRIES

\author{
FORMATION
}

\section{AMÉLIORATION, RENOUVELLEMENT}

DEUXIĖME ÉDTrion

Ornée de 67 figures

\section{PARIS}

LIBRAIRIE CENTRALE D'AGRICLLTURE ET DE JARDINAGK IUE DES ÉCOLES, 62 (ancien 82), PRÉS LE MUSÉE dE CLUNY - Auguste Gors, éditeur - 


\section{BIBLIOTHĖQUE DE L'AGRIGULTEUR PRATIGIEN}

ABEILLES (Éducation des), par A. EsPanet. In-18.............

AGRICULTURE. Théorie et pratique, par Murphy. 1 vol. in-18, fig........

AGRONOMIE ET PHYSIOLOGIE VÉGÉTALE. Études theoriques et pratiques, par 1. Pierre. 4 vol.in-18. T. Ier: Sol, Engrais, Amendements. - Tome II: Plantes fourragères; Graines et produits dérivés. - Tome ilI : Céréales. - Tome IV: Plantes industrielles; recherches diverses.............. 14 ALMANACH DE L'AGRICULTEUR PRATICIEN pour 1873.15e année.In-18, fig. Les années 1858 à 1872 chaque ................................ ANALYSE CHIMIQUE APPLIQUÉE A L'AGRICULTURE (Notions èlémen-

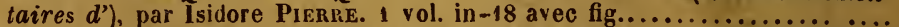
BASSE-COUR ET LAPIN DOMESTIQUE, par YSABEAU. 1 vol. in-18 .......... BÉTAIL (De l'alimentation du) par. Isidore Pierre, 4e édition. 1 vol. in-18..... BETES OVINES (Des) ET DES CHEVRES, par Ysabeac. 1 vol. in-18, fig. .... CAILLES, FAISANS ET PERDRIX, par Allary. 1 vol. in-18, fig........... GHEVAL. Principes sommaires d'élevage du cheval, par Basserie. $2^{e}$ édit. In-18. CHEVAUX. Conseils aux Éleveurs, par Ch. do HAys. 1 vol. in-18, fig.......... CHEVAUX DE GHASSE, leur condition en France par le comte Le Coutrulx,

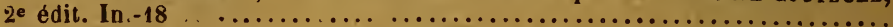

CHIENS. Maladies et traitement, par HERTwig. 2 édition. 1 vol. in-18... ..... ENGRAIS DE MER : Tangues, Trez, Merl, Goëmons, Débris divers de poissons, Guanos, etc., par Isidore Pierre, 2e édit. 1 vol. in-18...............

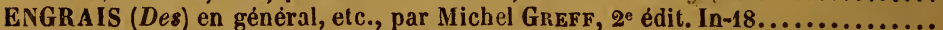
FAISANS, COLINS, CANARDS MANDARINS, etc., par A. Legrand. 1 vol. in-18. FoURRAGES (Valeur nutritive des), par Isidore Pierre. 4c édit. In-18...... FUMIER (Platrage ou sulfatage $d u$ ), par I. Pierne. $3^{e}$ édit. In-18............ GRAMINEES CERÉELES ET FOURRAGÈRES, rendement des diverses espèces; sols qui conviennent, etc., par DEMOOR. 1 vol. in-18 orné de 150 figures.

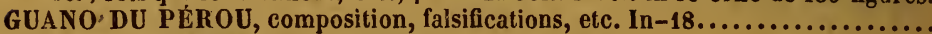

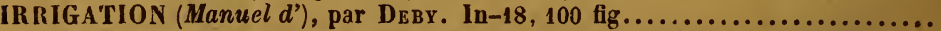

LAPIN DOMESTIQUE (Education $d u$ ), par Alexis ESPANET, $\mathfrak{5}^{\circ}$ éd. In-18.....

MAIS ET SORGHO SUCRE் (Alcoolisation des tiges de). Alcool.-Cidre.-Bière.

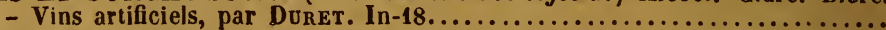

MARNE ET GHAUX. Leur emploi en agriculture, par Isidore Prerre. In-18... MATIÈRES FERTILISANTES. Choix, achat, emploi, origine, composition, va. leur, effets, durée, modes d'emploi, etc., par Dunoux. In-18..............

MÉDECINE VÉTÉRINAIRE DES BÊTES A CORNES, par CotTIER, in-18.... PIGEONS, Oiseaux de luxe, de volière et de cage, par A. EsPanet. $2^{e}$ éd. In-18. PLANTES FOURRAGERES (Traité pratique de la culture des), par de ThinR.

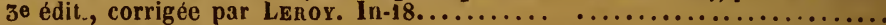

PORCIIERIES (De l'élablissement des), construction, etc., par GrandvoInNet.

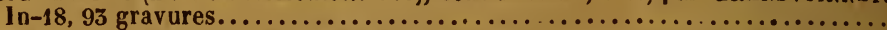

PORCS (Du traitement des) aux différentes époques de l'année. In-18, 64 grav. POULES, DINDES, OIES ET CANARDS, par Alexis EsPanet, 2e éd. In-18, fig. PRAIRIES ET FOURRAGES dans les terres fortes el argileuses du Midi (Traité

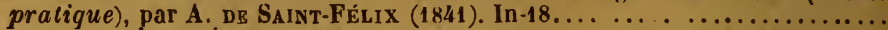
RÉCOLTES DÉROBÉES (Des), comme fourrages et engrais verts, et culture de la Moutarde Blanche, traduit de l'anglais par J. A. G. In-18, fig........... SANG DE RATE des animaux d'espèces ovine et bovine, par I. PIerre In-18.. SEMAILLES EN LIGNE (Des) et des semoirs mécaniques, par F. Georges. In-18. SORGHO A SUCRE. Culture, etc., par Madinier. In-8................... SORGHO A SUCRE (Guide du distillateur $d u$ ), par F. BoundaIs. In-18..... St abulation de l'espèce bovine, par Peers. In-18................... VÉGÉTAUX (Nutril. des) dans ses rapp. avec les Assolements, par DE BABO. In-18. VISITE à un véritable agriculteur praticien, par Durand-Sa voyat. In-18..... 





SMITHSONIAN INSTITUTION LIBRARIES

|| ||| |||||||||| || ||||| |||||| ||||||||||||||||||||||||||||||||||||||

39088006149769 